\title{
Etiopathogenesis of necrotizing enteroculitis
}

Citation for published version (APA):

Moonen, R. (2016). Etiopathogenesis of necrotizing enteroculitis: a point of view from vascular biology. [Doctoral Thesis, Maastricht University]. Maastricht University. https://doi.org/10.26481/dis.20161219rm

Document status and date:

Published: 01/01/2016

DOI:

10.26481/dis.20161219rm

Document Version:

Publisher's PDF, also known as Version of record

\section{Please check the document version of this publication:}

- A submitted manuscript is the version of the article upon submission and before peer-review. There can be important differences between the submitted version and the official published version of record.

People interested in the research are advised to contact the author for the final version of the publication, or visit the DOI to the publisher's website.

- The final author version and the galley proof are versions of the publication after peer review.

- The final published version features the final layout of the paper including the volume, issue and page numbers.

Link to publication

\footnotetext{
General rights rights.

- You may freely distribute the URL identifying the publication in the public portal. please follow below link for the End User Agreement:

www.umlib.nl/taverne-license

Take down policy

If you believe that this document breaches copyright please contact us at:

repository@maastrichtuniversity.nl

providing details and we will investigate your claim.
}

Copyright and moral rights for the publications made accessible in the public portal are retained by the authors and/or other copyright owners and it is a condition of accessing publications that users recognise and abide by the legal requirements associated with these

- Users may download and print one copy of any publication from the public portal for the purpose of private study or research.

- You may not further distribute the material or use it for any profit-making activity or commercial gain

If the publication is distributed under the terms of Article $25 \mathrm{fa}$ of the Dutch Copyright Act, indicated by the "Taverne" license above, 
ETIOPATHOGENESIS OF NECROTIZING ENTEROCOLITIS:

A Point of View from Vascular Biology

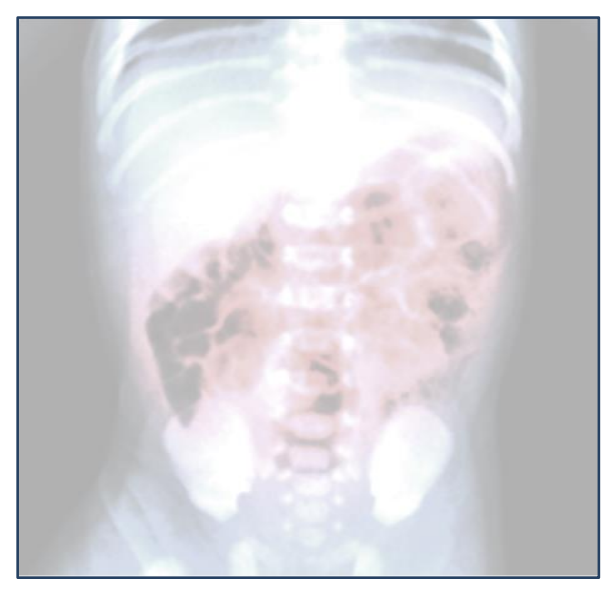


Etiopathogenesis of Necrotizing Enterocolitis: A Point of View from Vascular Biology

Thesis, Maastricht University, Maastricht, The Netherlands

ISBN: 978-94-6233-481-6

Design and layout: R.M.J. Moonen

Printed by: Gildeprint

Copyright @ 2016 R.M.J. Moonen, Brunssum, The Netherlands

All rights reserved. No part of this book may be reproduced, stored in a retrievel system, or transmitted, in any form or by any means, electronic, mechanical, photocopying, recording or otherwise, without the prior written permission of the holder of the copyright. 


\title{
ETIOPATHOGENESIS OF NECROTIZING ENTEROCOLITIS:
} A Point of View from Vascular Biology

\author{
PROEFSCHRIFT \\ Ter verkrijging van de graad van doctor aan de Universiteit Maastricht, \\ op gezag van Rector Magnificus, Prof. dr. Rianne M. Letschert, \\ volgens het besluit van het College van Decanen, \\ openbaar te verdedigen op maandag 19 december 2016 om 14.00 uur
}

door

Rob Maria Johannes Moonen

Geboren op 25 mei 1975 te Heerlen 


\section{Promotores}

Prof. dr. L.J.I. Zimmermann

Prof. dr. F. Pérez-Vizcaino (Universidad Complutense de Madrid, Spain)

Prof. dr. B.W. Kramer

\section{Co-promotor}

Dr. E. Villamor

\section{Beoordelingscommissie}

Prof. dr. F.C. Ramaekers (voorzitter)

Prof. dr. E. Dompeling

Prof. dr. D.A. Giussani (University of Cambridge, United Kingdom)

Prof. dr. F.W. Prinzen

Prof. dr. M. Vento (University \& Polythenic Hospital La Fe, Valencia, Spain)

Financial support by Hero Baby, AbbVie BV and Nutricia Early life Nutrition for the publication of this thesis is gratefully acknowledged. 


\section{TABLE OF CONTENTS}

Chapter $1 \quad$ Justification, aim and outline of this thesis

\section{PART I DEVELOPMENTAL CHANGES IN MESENTERIC ARTERIAL REACTIVITY UNDER NORMAL AND PATHOLOGICAL CONDITIONS}
Chapter 2 Developmental changes in mesenteric artery reactivity in embryonic and newly hatched chicks
Chapter $3 \quad$ Mesenteric artery reactivity and small Intestine morphology in a chicken model of hypoxia-Induced fetal growth restriction
Chapter 4 Hypoxia-induced contraction of chicken embryo mesenteric arteries: mechanisms and developmental changes

\section{PART II THE L-ARGININE/NO PATHWAY AND THE RISK OF NEC}

Chapter 5 Carbamoyl-phosphate synthetase polymorphisms as a risk factor for necrotizing enterocolitis

Chapter 6 Association between the p.Thr1406Asn polymorphism of the carbamoyl-phosphate synthase 1 gene and necrotizing enterocolitis: A prospective multicenter study

Chapter 7 The T1405N carbamoyl phosphate synthetase polymorphism does not affect plasma arginine concentrations and in preterm infants

Chapter $8 \quad$ Summary, general discussion and future perspectives

$\begin{array}{lll}\text { Chapter } 9 & \text { Nederlandse samenvatting } & 201\end{array}$

$\begin{array}{lll}\text { Chapter } 10 & \text { Valorisation addendum } & 207\end{array}$

Abbreviations $\quad 215$

$\begin{array}{ll}\text { Co-authors } & 219\end{array}$

Dankwoord (Acknowledgements) 223

About the author: curriculum vitae and publications 227 
Chapter 1

JUSTIFICATION, AIM AND OUTLINE OF THIS THESIS

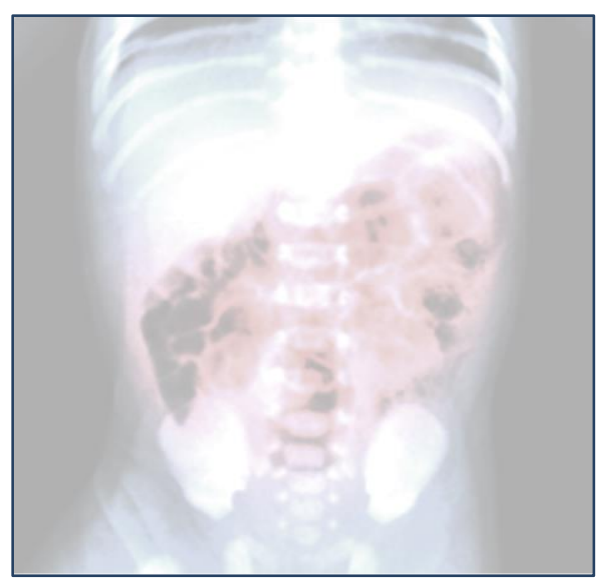


Necrotizing enterocolitis (NEC) is an inflammatory intestinal disorder primarily seen in preterm infants, characterized by variable damage to the intestinal tract, ranging from mucosal injury to full-thickness necrosis and perforation. ${ }^{1-5} \mathrm{NEC}$ remains a leading cause of morbidity and mortality in neonatal intensive care units The overall incidence of NEC is about 1 in 1000 live births, but occurs in up to $7-11 \%$ of very low birth weight (VLBW, birth weight $<1500 \mathrm{~g}$ ) infants. Mortality is $15-30 \%$ and is higher with lower birth weight and earlier gestational age. Survivors may be left with significant sequelae, which include not only gastrointestinal complications, such as a short gut syndrome, but also severe neurodevelopmental delay. ${ }^{1}$ The incidence rates of NEC have increased over the last decades, largely attributable to an increased survival of extremely preterm, who are at the highest risk of developing NEC. ${ }^{1-5}$

The natural history of NEC is a fulminant progression with severe inflammation of the bowel, leading to septic shock, multiorgan failure, and death within hours or days of onset if appropriate treatment is not timely instituted. Therefore, early detection and initiation of treatment in NEC are key factors in its course and prognosis. Term infants who develop NEC tend to have specific risk factors such as congenital heart disease, sepsis, or hypotension. ${ }^{2,4}$ In contrast, the pathogenesis of NEC in very preterm infants remains elusive and is likely multifactorial. Suspected ethiopathogenic mechanisms include the developmental immaturity of gastrointestinal motility, digestive ability, circulatory regulation, intestinal barrier function, and immune defence. Immaturity of these functions predisposes the premature infant to intestinal injury and to inappropriate responses to injury (Fig.1).5,6 


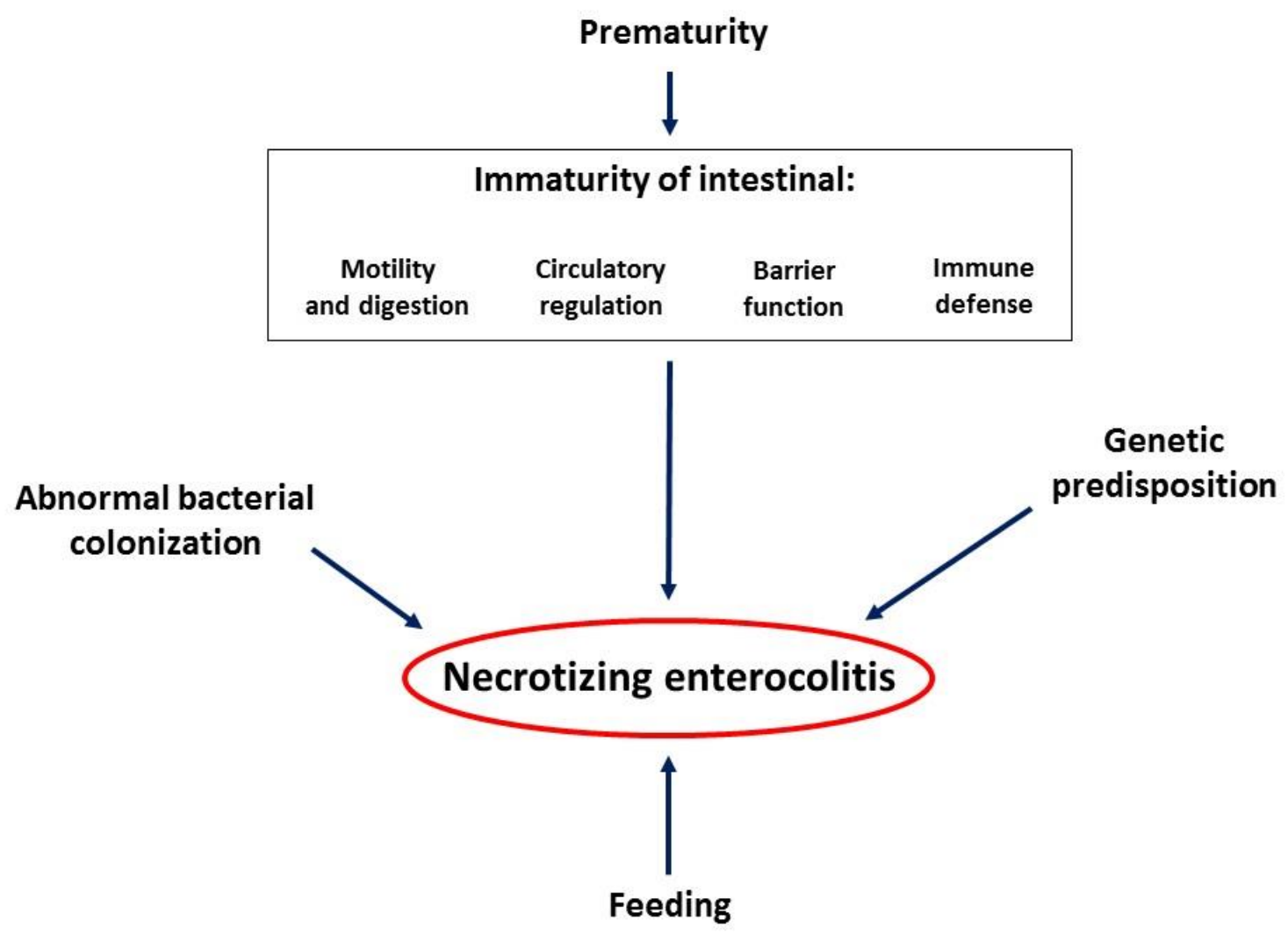

Figure 1. Suspected etiopathogenic mechanisms in NEC (adapted from: Lin et al, 2008 seminars in perinatology. ${ }^{6}$ )

Taken into account the multifaceted character of its etiopathogenesis, NEC can be seen, as an intrinsic intestinal, an immunological, an inflammatory, an infectious, or a vascular disease. The present thesis focus on this last perspective.

\section{NEC AS VASCULAR DISEASE}

Intestinal ischemia is one of the main factors contributing to the pathogenesis of NEC. This is supported by the fact that the histopathologic hallmark of NEC is coagulation necrosis, particularly within the muscle layers of the intestine. ${ }^{7}$ However, as stated by Reber et al., the question is not whether ischemia occurs in the pathogenesis of NEC, but rather, when during the course of disease progression ischemia occurs. ${ }^{7}$ In other words, whether the hypoxic-ischemic insult triggers the development of NEC or is the result of the inflammatory process is still a matter of debate. ${ }^{5,8-10}$ An adequate knowledge of the particular characteristics of the fetal and neonatal intestinal circulation is necessary to understand its possible role in the pathogenesis of NEC. 
During prenatal life, the placenta is the organ responsible for nutrition. Consequently, the fetal intestine is a relatively dormant organ which presents a low-flow, highresistance vascular bed that is adequate to meet its limited tissue $\mathrm{O}_{2}$ demand. $7,11,12$ After birth, the intestine becomes the sole site for nutrient absorption and the rate of intestinal growth increases, requiring a dramatic increase in blood flow. On the other hand, intestinal development occurs while the new forming vessels are simultaneously acquiring the capacity to regulate vascular tone and undergoing changes in the contractile apparatus, the density of receptors and the signal transduction pathways leading to contraction/relaxation. ${ }^{13}$ Preterm birth takes place when this developmental process is far to be completed and, consequently, the intestinal vessels need to cope with the new circulatory situation with the level of maturity of a fetal vessel. In addition, the low vascular resistance characteristic of newborn intestine alters the capacity of this vasculature to respond to systemic circulatory perturbations, such as hypotension and arterial hypoxemia. ${ }^{7}$

The control of intestinal vascular resistance is a complex process of interaction between extrinsic and intrinsic control mechanisms. Extrinsic vascular regulation is mediated by effector mechanisms such as the autonomic nervous system. Through these extrinsic mechanisms the intestinal circulation is integrated into systemic cardiovascular reflexes and typically function to preserve systemic cardiovascular homeostasis, often at the expense of the local intestinal circulation. Intrinsic vascular regulation is mediated by effector mechanisms produced and released within the intestine and its microcirculation. These mechanisms serve to maintain the delivery of oxygen and nutrients to the intestinal tissue and preserve hemodynamic homeostasis locally within the intestinal microcirculation. A balance between two intrinsic stimuli, one vasoconstrictor, endothelin-1 (ET-1) and one vasodilator, nitric oxide (NO), appears to be the predominate stimulus controlling intestinal vascular resistance in the fetus and the newborn. ${ }^{10}$ However, our current understanding of the fetal and neonatal intestinal vascular physiology and pathophysiology is limited. One of the reasons for this limitation is the use of mammalian models, which are complex because the fetal/placental circulation has to be exposed to intervention only through complex surgery and experimental manipulations affect both the mother and the fetus. ${ }^{14,15}$ Therefore, only a limited number of observations have been made on the development 
of fetal intestinal circulation in mammals ${ }^{11,16}$ and there is a need for additional models, addressing the limitations of mammalian models. ${ }^{15}$

The hope for future targeted therapies and for the prevention of NEC requires knowledge of the fundamental mechanisms controlling intestinal development under normal and pathological conditions. As mentioned above, the present thesis focus on role of the vascular system in the pathogenesis of NEC and approached the problem from two perspectives. In the first part, we investigated the developmental changes in mesenteric arterial reactivity. In the second part, we analyzed the role of L-arginine, the precursor for the synthesis of NO, on the pathogenesis of NEC.

\section{AIMS AND OUTLINE}

\section{PART I: DEVELOPMENTAL CHANGES IN MESENTERIC ARTERIAL REACTIVITY UNDER NORMAL AND PATHOLOGICAL CONDITIONS}

An optimal balance between mesenteric vasoconstrictor and dilator forces is required to meet postnatal metabolic demands, and any imbalance may contribute to intestinal ischemia and predispose preterm infants to NEC. The aim of the first part of this thesis was to delve into the development of mesenteric vascular reactivity under physiological and pathological conditions. For this purpose we used the chicken embryo as experimental model.

In chapter 2, we investigated the ontogeny of the responsiveness of mesenteric arteries from chicken embryos and hatchlings to constrictor and relaxant agonists.

In chapter 3, we analyzed how incubation under chronic hypoxia affected the development of mesenteric arterial reactivity and intestinal morphology in the chicken embryo.

In chapter 4, we analyzed the developmental changes and the mechanisms involved in the response of chicken embryo mesenteric arteries to acute hypoxia. 


\section{PART II: THE L-ARGININE/NO PATHWAY AND THE RISK OF NEC}

As mentioned above, NO appears to be the main vasodilator participating in the control of fetal and neonatal mesenteric circulation. Moreover, besides its role in the regulation of intestinal blood flow, NO plays a key role in the maintenance of mucosal integrity, intestinal barrier function, and post-injury intestinal reparation. ${ }^{7,17-19}$ L-arginine is the substrate for NO production and several studies demonstrated that plasma arginine concentrations are decreased in infants with NEC. ${ }^{20-25}$ Nevertheless, the mechanisms explaining this relative deficiency of $L$-arginine in infants with NEC are far from being understood.

Arginine is a urea cycle intermediate. The first step in the urea cycle occurs inside the mitochondrion and is catalyzed by the enzyme, carbamoyl-phosphate synthetase I (CPS1). ${ }^{26}$ A number of functional single nucleotide polymorphisms (SNPs) have been identified in the CPS1 gene. ${ }^{26-28}$ One of those SNPs (p.Thr1406Asn also published as T1405N; rs1047891 formerly designated as rs7422339) has been linked to functional consequences affecting the downstream availability of urea-cycle intermediates, including L-arginine. ${ }^{26-28}$ Several studies demonstrated the association of the CPS1 p.Thr1406Asn genotype with clinical situations where endogenous NO production is critically important. ${ }^{29-32}$

We hypothesized that variations in the p.Thr1406Asn genotype are associated with the risk of developping NEC. This hypothesis was initially tested in a retrospective casecontrol study (chapter 4) and proved to be true. In oder to confirm the results of the retrospective study, we conducted a multicenter, prospective cohort study in which the association between the p.Thr1406Asn genotype and NEC risk (chapter 5) and L-arginine levels (chapter 6) were investigated. 


\section{REFERENCES}

1 Neu, J. Necrotizing enterocolitis: the mystery goes on. Neonatology 106, 289295 (2014).

2 Berman, L. \& Moss, R. L. Necrotizing enterocolitis: an update. Seminars in fetal \& neonatal medicine 16, 145-150 (2011).

3 Choi, Y. Y. Necrotizing enterocolitis in newborns: update in pathophysiology and newly emerging therapeutic strategies. Korean J Pediatr 57, 505-513 (2014).

4 Dominguez, K. M. \& Moss, R. L. Necrotizing enterocolitis. Clinics in perinatology 39, 387-401 (2012).

5 Lim, J. C., Golden, J. M. \& Ford, H. R. Pathogenesis of neonatal necrotizing enterocolitis. Pediatr Surg Int 31, 509-518 (2015).

6 Lin, P. W., Nasr, T. R. \& Stoll, B. J. Necrotizing enterocolitis: recent scientific advances in pathophysiology and prevention. Semin Perinato/32, 70-82 (2008).

7 Reber, K. M., Nankervis, C. A. \& Nowicki, P. T. Newborn intestinal circulation. Physiology and pathophysiology. Clinics in perinatology 29, 23-39 (2002).

8 Nankervis, C. A., Giannone, P. J. \& Reber, K. M. The neonatal intestinal vasculature: contributing factors to necrotizing enterocolitis. Semin Perinato/32, 83-91 (2008).

9 Zhang, H. Y., Wang, F. \& Feng, J. X. Intestinal microcirculatory dysfunction and neonatal necrotizing enterocolitis. Chin Med J (Engl) 126, 1771-1778 (2013).

10 Watkins, D. J. \& Besner, G. E. The role of the intestinal microcirculation in necrotizing enterocolitis. Seminars in pediatric surgery 22, 83-87 (2013).

11 Edelstone, D. I. \& Holzman, I. R. Fetal intestinal oxygen consumption at various levels of oxygenation. Am J Physiol 242, H50-54 (1982).

12 Nankervis, C. A., Reber, K. M. \& Nowicki, P. T. Age-dependent changes in the postnatal intestinal microcirculation. Microcirculation 8, 377-387 (2001).

13 Altimiras, J., Crossley, D. A. \& Villamor, E. in Cardio-Respiratory Control in Vertebrates. Comparative and Evolutionary Aspects Vol. 3 (eds M.L. Glass \& S.C. Wood) 397-427 (Springer-Verlag 2009).

14 Ruijtenbeek, K., De Mey, J. G. \& Blanco, C. E. The chicken embryo in developmental physiology of the cardiovascular system: a traditional model with 
new possibilities. Am J Physiol Regul Integr Comp Physiol 283, R549-550; author reply R550-541 (2002).

15 Sutendra, G. \& Michelakis, E. D. The chicken embryo as a model for ductus arteriosus developmental biology: cracking into new territory. Am J Physiol Regul Integr Comp Physiol 292, R481-484 (2007).

16 Wolfs, T. G. et al. Endotoxin induced chorioamnionitis prevents intestinal development during gestation in fetal sheep. PloS one 4, e5837 (2009).

17 Upperman, J. S. et al. Mechanisms of nitric oxide-mediated intestinal barrier failure in necrotizing enterocolitis. Seminars in pediatric surgery 14, 159-166 (2005).

18 Chokshi, N. K. et al. The role of nitric oxide in intestinal epithelial injury and restitution in neonatal necrotizing enterocolitis. Seminars in perinatology 32, 9299 (2008).

19 Nowicki, P. T. et al. Intestinal O2 consumption in necrotizing enterocolitis: role of nitric oxide. Pediatric research 59, 500-505 (2006).

20 Amin, H. J. et al. Arginine supplementation prevents necrotizing enterocolitis in the premature infant. J Pediatr 140, 425-431 (2002).

21 Becker, R. M. et al. Reduced serum amino acid concentrations in infants with necrotizing enterocolitis. J Pediatr 137, 785-793 (2000).

22 Celik, I. H., Demirel, G., Canpolat, F. E. \& Dilmen, U. Reduced plasma citrulline levels in low birth weight infants with necrotizing enterocolitis. Journal of clinical laboratory analysis 27, 328-332 (2013).

23 Richir, M. C. et al. Low plasma concentrations of arginine and asymmetric dimethylarginine in premature infants with necrotizing enterocolitis. Br J Nutr 97, 906-911 (2007).

24 Wu, G., Jaeger, L. A., Bazer, F. W. \& Rhoads, J. M. Arginine deficiency in preterm infants: biochemical mechanisms and nutritional implications. The Journal of nutritional biochemistry 15, 442-451 (2004).

25 Zamora, S. A. et al. Plasma L-arginine concentrations in premature infants with necrotizing enterocolitis. J Pediatr 131, 226-232 (1997).

26 Martinez, A. I., Perez-Arellano, I., Pekkala, S., Barcelona, B. \& Cervera, J. Genetic, structural and biochemical basis of carbamoyl phosphate synthetase 1 deficiency. Molecular genetics and metabolism 101, 311-323 (2010). 
27 Summar, M. L. et al. Environmentally determined genetic expression: clinical correlates with molecular variants of carbamyl phosphate synthetase I. Molecular genetics and metabolism 81 Suppl 1, S12-19 (2004).

28 Summar, M. L. et al. Characterization of genomic structure and polymorphisms in the human carbamyl phosphate synthetase I gene. Gene 311, 51-57 (2003).

29 Pearson, D. L. et al. Neonatal pulmonary hypertension--urea-cycle intermediates, nitric oxide production, and carbamoyl-phosphate synthetase function. The New England journal of medicine 344, 1832-1838 (2001).

30 Summar, M. L. et al. Relationship between carbamoyl-phosphate synthetase genotype and systemic vascular function. Hypertension 43, 186-191 (2004).

31 Kallianpur, A. R. et al. The hemochromatosis C282Y allele: a risk factor for hepatic veno-occlusive disease after hematopoietic stem cell transplantation. Bone marrow transplantation 35, 1155-1164 (2005).

32 Canter, J. A. et al. Genetic variation in the mitochondrial enzyme carbamylphosphate synthetase I predisposes children to increased pulmonary artery pressure following surgical repair of congenital heart defects: a validated genetic association study. Mitochondrion 7, 204-210 (2007). 


\section{PART I}

DEVELOPMENTAL CHANGES IN MESENTERIC ARTERIAL REACTIVITY UNDER NORMAL AND PATHOLOGICAL CONDITIONS

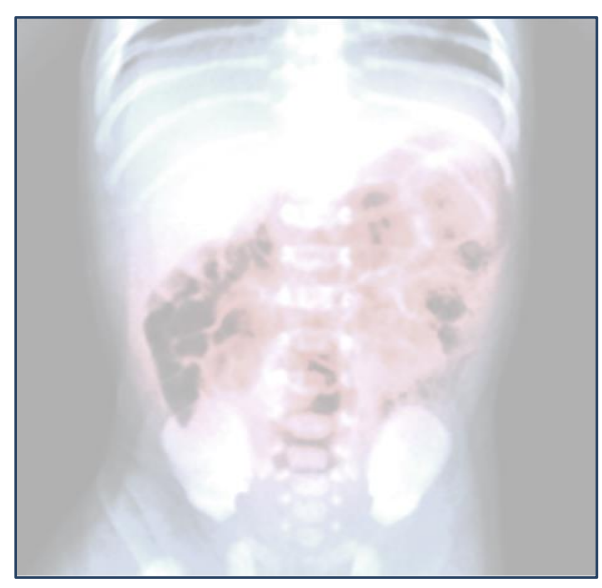


Chapter 2

\section{DEVELOPMENTAL CHANGES IN MESENTERIC ARTERY REACTIVITY IN EMBRYONIC AND NEWLY HATCHED CHICKS}

Rob M.J. Moonen and Eduardo Villamor

J Comp Physiol B 181, 1063-1073 (2011)

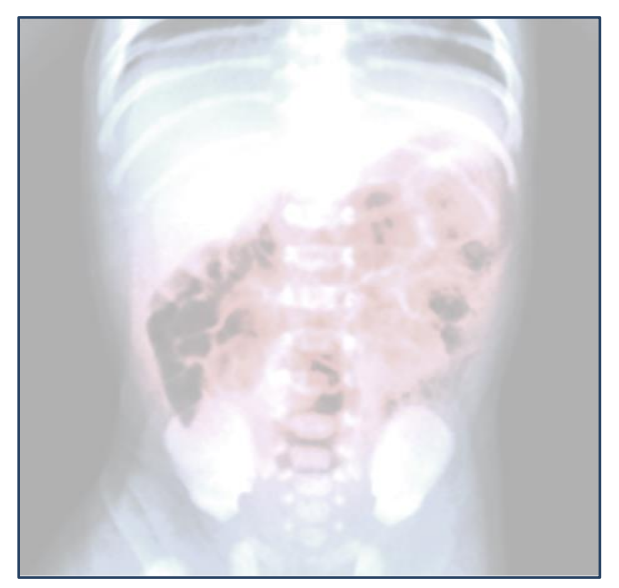




\section{ABSTRACT}

At birth, the intestine becomes the sole site for nutrient absorption requiring a dramatic increase in blood flow. The vascular changes accompanying this transition have been partly characterized in mammals. We investigated, using wire myography, the developmental changes in chick mesenteric artery (MA) reactivity. Rings of the MA from 15-day (E15) and 19-day (E19) chicken embryos (total incubation 21 days) as well as non-fed 0-3-h-old ( $\mathrm{NH3h}$ ) and first-fed 1-day-old ( $\mathrm{NH1d}$ ) newly hatched chicks contracted in response to $\mathrm{KCl}$, norepinephrine (NE), U46619, and endothelin (ET)-1 and relaxed in response to acetylcholine (ACh), sodium nitroprusside (SNP), and forskolin, indicating the presence of electro- and pharmaco-mechanical coupling as well as CGMP- and CAMP-mediated relaxation. In ovo development and transition to ex-ovo life was accompanied by alterations in the response of the MAs but a different developmental trajectory was observed for each reactivity pathway tested. Thus, the contractile efficacy of $\mathrm{KCl}$ underwent a linear increase (E15 $<\mathrm{E} 19<\mathrm{NH} 3 \mathrm{~h}<\mathrm{NH} 1 \mathrm{~d})$. The efficacy of NE and U46619 increased in ovo but not ex ovo $(\mathrm{E} 15<\mathrm{E} 19=\mathrm{NH} 3 \mathrm{~h}=$ $\mathrm{NH} 1 \mathrm{~d})$ and the efficacy of ET-1 peaked at $\mathrm{E} 19(\mathrm{E} 15<\mathrm{E} 19>\mathrm{NH} 3 \mathrm{~h}=\mathrm{NH} 1 \mathrm{~d})$. The relaxations elicited by ACh (endothelium-dependent), SNP, and forskolin did not undergo significant developmental changes. In conclusion, the ability of chick MAs to constrict in response to pharmacological stimuli increases during the embryonic period, but no dramatic changes are induced by hatching or the first feeding. Maturation of vasodilator mechanisms precedes that of vasoconstrictor mechanisms. Alterations of the delicate balance between vasoconstrictors and vasodilators may play an important role in perinatal intestinal diseases. 


\section{INTRODUCTION}

The placenta is the organ responsible for nutrition of the mammalian fetus. Consequently, the fetal intestine is a relatively dormant organ which presents a lowflow, high-resistance vascular bed that is adequate to meet its limited tissue $\mathrm{O}_{2}$ demand. ${ }^{1-3}$ After birth, the intestine becomes the sole site for nutrient absorption and the rate of intestinal growth increases, requiring a dramatic increase in blood flow. The intestinal vascular changes that accompany the transition between the fetal and the neonatal life have been characterized in several mammalian species, including pig and sheep. ${ }^{1-4}$

The most pressing reason to study the fetal and neonatal intestinal circulation is to clarify its putative role in the pathogenesis of necrotizing enterocolitis (NEC). ${ }^{5} \mathrm{NEC}$ is the most common life-threatening gastrointestinal emergency encountered in the neonatal intensive care unit. The pathophysiology of NEC remains elusive and is likely multifactorial. ${ }^{5-8}$ Recently, it has been proposed that a misbalance between vasoconstriction and vasodilatation in the immature intestine may predispose to NEC. ${ }^{5,9}$ However, only a limited number of observations have been made on the development of fetal intestinal circulation in mammals ${ }^{1,8}$ because mammalian models are technically complex and experimental manipulations affect both the mother and the fetus. ${ }^{10,11}$ Therefore, there is a need for additional models, addressing these limitations. ${ }^{10,11}$

In the last few years, the chicken embryo has emerged as a suitable model for studying developmental vascular biology..$^{10-20}$ In contrast to mammals, which depend on a continuous transference of nutrients from the maternal circulation to the developing fetus, all the nutrients required for the formation and growth of the chicken embryo are pre-packaged in the egg at the time of laying. ${ }^{21}$ Glucose and aminoacids form the main energy source during the first stages of chicken embryo development but later the lipids of the yolk represent the primary nutrient source, providing over $90 \%$ of the energy required. ${ }^{21,22}$ Yolk lipids and other constituents are transferred to the embryonic circulation through the yolk sac membrane, a highly vascularised structure which grows outward from the embryo to envelope the whole of the yolk. ${ }^{21}$ Towards the end 
of incubation, the remaining yolk is internalized into the abdominal cavity and provides nutrient reserves for several days. ${ }^{23,24}$ In the hatching chick, yolk is utilized simultaneously via two routes. One route is by phagocytosis or endocytosis of yolk contents to the circulation, while the other is by transport through the yolk stalk into the small intestine. ${ }^{24}$ However, chicks are precocial and directly forage for exogenous feed. ${ }^{25}$ Therefore, the immediate posthatch period is characterized by a transition from the use of lipid-rich yolk as the nutrient source to exogenous feed rich in carbohydrates and proteins. ${ }^{23,24,26}$ This transition is accompanied by rapid physical and functional development of the gastrointestinal tract, ${ }^{25,27}$ but its effects on the intestinal circulation have not been studied so far.

In a previous study, Rouwet et al. ${ }^{16}$ characterized, using intravital microscopy, the vasomotor responses of mesenteric arteries from 13 and 17-day-old chicken embryos (total incubation time 21 days). They observed that acetylcholine (ACh)-induced relaxation and norepinephrine (NE)- and hypoxia-induced contraction were already present at these developmental stages. The ability of mesenteric arteries to constrict increased with development, whereas vasodilator responses remained unchanged. ${ }^{16}$ Thus the study of Rouwet et al. demonstrated the presence of pharmacomechanical coupling in the chicken embryo mesenteric vasculature but, unfortunately, they did not examine a larger number of vasoactive agonists and only compared two relative immature vessels without analyzing further maturation. In the present study, we hypothesized that the chicken mesenteric vascular reactivity undergoes further developmental changes during transition to ex ovo life and, more particularly, after the first feed. To test our hypothesis, we analyzed the response of mesenteric arteries from chicken embryos (15 and 19 days) and hatchlings (<3-h-old and 1-day-old) to constrictor and relaxant agonists. 


\section{METHODS}

\section{INCUBATION OF CHICKEN (GALLUS GALLUS) EMBRYOS AND VESSEL ISOLATION}

All experimental procedures were carried out in accordance with the Dutch Law on Animal Experimentation and the European Directive for the Protection of Vertebrate Animals Used for Experimental and Other Scientific Purposes (86/609/EU) and approved by the Committee on Animal Experimentation of the University of Maastricht. Fertilized eggs from White Leghorn chickens ('t Anker, Ochten, The Netherlands) were incubated at $37.8^{\circ} \mathrm{C}, 45 \%$ humidity and rotated once per hour over an angle of $90^{\circ}$ (Incubator model 25HS, Masalles Comercial, Spain). At day 15 (E15) or 19 (E19) of the 21 days of incubation some embryos were used for experiments and others allowed to hatch. Hatchlings were used within the first $3 \mathrm{~h}(\mathrm{NH} 3 \mathrm{~h})$ or transferred to a brooder unit under $23 \mathrm{~h} /$ day of continuous light and temperature of $35^{\circ} \mathrm{C}$ and provided ad libitum access to water and a standard starter diet. ${ }^{20}$ This last group of chicks was studied within the second day post-hatch ( $\mathrm{NH} 1 \mathrm{~d})$.

On the experimental day, the animals were killed by decapitation, placed on the dorsal side on a Petri-dish coated with silicon and a midline laparotomie and sternotomy were performed. With the aid of a dissecting microscope, the cranial MA was carefully dissected free from surrounding tissue. The cranial MA is the vessel which supplies most of chicken intestine and also the yolk sac during the embryonic period. ${ }^{28}$ Only the intestinal part of the artery (i.e. the part distal to the emergence of the branch which supplies the yolk sac) was used in our study.

\section{RECORDING OF ARTERIAL REACTIVITY}

Two stainless steel wires (diameter $40 \mu \mathrm{m}$ ) were inserted into the lumen of the vessels, which were mounted as a 1.7-2 mm length ring segment between an isometric force transducer and a displacement device in a myograph (Danish Myo Technology A/S model 610M, Aarhus, Denmark). The segment lengths were determined with the aid of an eyepiece scale which was incorporated in the dissecting microscope. The myograph organ bath $(5 \mathrm{~mL}$ vol) was filled with Krebs-Ringer bicarbonate (KRB, composition in mmol L-1: $\mathrm{NaCl}, 118.5 ; \mathrm{KCl}, 4.75 ; \mathrm{MgSO}_{4} \cdot 7 \mathrm{H}_{2} \mathrm{O}, 1.2 ; \mathrm{KH}_{2} \mathrm{PO}_{4}, 1.2$; $\mathrm{NaHCO}_{3}, 25.0 ; \mathrm{CaCl}_{2}, 2.5$; glucose, 5.5) buffer maintained at $39^{\circ} \mathrm{C}$ and aerated with 
95\% $\mathrm{O}_{2} / 5 \% \mathrm{CO}_{2}$ (Po2 $82.3 \mathrm{kPa}, \mathrm{SD} 6.9, \mathrm{n}=12$, measured with an $\mathrm{ABL} 510$ blood gas analyzer, Radiometer Copenhagen, Denmark). After an equilibration period of $30 \mathrm{~min}$, the vessels were distended to their individual optimal diameter, which evoked a resting tension corresponding to a transmural pressure of $10 \mathrm{mmHg}$ (E15) or $20 \mathrm{mmHg}$ (E19 and hatchlings). These pressures correspond to the mean arterial blood pressure reported in chicken at the corresponding age ${ }^{29}$ and elicited the highest contractile response to $\mathrm{KCl}$, as determined in pilot experiments. After $30 \mathrm{~min}$ of incubation under resting tension, a control reference contraction was elicited by raising the $\mathrm{K}^{+}$ concentration of the buffer $(62.5 \mathrm{mM})$ in exchange for $\mathrm{Na}^{+}$.

\section{CONTRACTILE RESPONSES}

Concentration-response curves to $\mathrm{KCl}(31.25$ - $125 \mathrm{mM})$, the nonselective adrenergic receptor agonist $\mathrm{NE}(10 \mathrm{nM}-0.1 \mathrm{mM})$, the thromboxane/prostaglandin $\mathrm{H}_{2}$ (TP) receptor agonist U-46619 (10 nM-3 $\mu \mathrm{M})$, and endothelin (ET)-1 (0.1 nM-0.1 $\mu \mathrm{M})$ were constructed by increasing the organ chamber concentration of the drug, by cumulative increments after a steady-state response had been reached with each increment. When two or more agonists were studied in the same arterial preparation, the vessels were repeatedly washed and allowed to equilibrate for at least $30 \mathrm{~min}$. If the tone did not recover to resting level, the vessels were discarded for further experiments.

\section{RELAXANT RESPONSES}

Concentration-response curves to relaxants were constructed following establishment of stable contraction with $\mathrm{NE}(10 \mu \mathrm{M})$ or $\mathrm{KCl}(62.5 \mathrm{mM})$. The muscarinic receptor agonist acetylcholine (ACh, $10 \mathrm{nM}-0.1 \mathrm{mM})$, the nitric oxide (NO) donor sodium nitroprusside (SNP, $10 \mathrm{nM}-0.1 \mathrm{mM}$ ) and the adenylate cyclase activator forskolin $(10 \mathrm{nM}-10 \mu \mathrm{M})$ were studied. Some experiments were performed in endothelium- denuded vessels. The endothelium was removed by gentle rubbing of the vessel lumen with a horse tail hair and its effective removal was verified in pilot experiments by scanning electron microscopy, as previously described. ${ }^{14,30}$ Other experiments were performed in the presence of the NO synthase inhibitor N $\omega$-Nitro-Larginine methyl ester (L-NAME, $0.1 \mathrm{mM}$ ), or the soluble guanylate cyclase (sGC) inhibitor $1 \mathrm{H}[1,2,4]$ oxadiazolo[4,3-a]quinoxalin-1-one (ODQ, $10 \mu \mathrm{M})$. 


\section{DRUGS AND SOLUTIONS}

Solutions containing different concentrations of $\mathrm{K}^{+}$were prepared by replacing part of the $\mathrm{NaCl}$ of the $\mathrm{KRB}$ buffer by an equimolar amount of $\mathrm{KCl}$. Arterenol bitartrate $(\mathrm{NE})$, ACh, SNP, L-NAME, L-arginine and forskolin were obtained from Sigma (St. Louis, MO); U-46619 was from Cayman Chemical (Ann Arbor, MI), and ODQ was from Tocris (Ballwin, MO). All drugs were dissolved initially in distilled deionized water (except U46619 and ODQ in DMSO and forskolin in ethanol) to prepare adequate stock solutions and further dilutions were also made in deionized water. The final bath concentration of DMSO or ethanol did not exceed $0.1 \%$ and had no effect on mechanical activity.

\section{DATA ANALYSIS}

Results are shown as mean (SD) of measurements in $n$ animals. For clarity, results are shown in the figures as mean \pm SEM. Contractions are expressed in terms of active wall tension $(\mathrm{mN} / \mathrm{mm}$, calculated as the force divided by twice the length of the segment) or as a percentage of the reference contraction to $\mathrm{KCl}(62.5 \mathrm{mM})$ performed for each individual ring at the beginning of the experiment. The relaxant responses are expressed as the percentage of reduction of the contraction induced by $\mathrm{NE}$ or $\mathrm{KCl}$. Sensitivity/potency (expressed as $p \mathrm{D}_{2}=-\log \mathrm{EC}_{50}$ ) and efficacy (expressed as $E_{\max }$ ) were calculated by nonlinear regression analysis of the concentration-response curves. In the case of several contractile agonists, a maximal effect was not reached, not allowing the calculation of the $p \mathrm{D}_{2}$. Thus, the $\mathrm{ED}_{20}$ (concentration of agonist causing a contraction representing $20 \%$ of the reference contraction to $62.5 \mathrm{mM} \mathrm{KCl}$ ) was calculated using nonlinear regression. ${ }^{31}$ The $-\log E D_{20}$ was used to express the potency of NE, ET-1 and U46619. Differences between mean values were assessed by one-way ANOVA followed by Bonferroni's post hoc $t$ test. Differences were considered significant at a $P<0.05$. All analyses were performed using GraphPad Prism (version 5.00 for Windows, GraphPad Software, San Diego California USA, http://www.graphpad.com). 


\section{RESULTS}

The mean body mass of the animals was E15: $12.99 \mathrm{~g}$ (SD 0.93, $n=40, \mathrm{P}<0.001$ vs. the other ages), E19: $27.97 \mathrm{~g}$ (SD 2.85, $n=47, P<0.001$ vs. NH3h and NH1d); NH3h: $41.82 \mathrm{~g}$ (SD 3.16, $n=50$ ); and NH1d: $40.25 \mathrm{~g}$ (SD 2.92, $n=16$ ). The mean optimal diameter of the MA rings did not significantly change with age (E15: $716 \mu \mathrm{m}$, SD 105, $n=40$; E19: $702 \mu \mathrm{m}$, SD 117, $n=47$; NH3h: $707 \mu \mathrm{m}$, SD 94, $n=50$; NH1d: $653 \mu \mathrm{m}$, SD $73, n=16)$.

\section{CONTRACTILE RESPONSES}

$\mathrm{KCl}$ (31.25 - $125 \mathrm{mM}$, Fig. 1), NE (10 nM - $0.1 \mathrm{mM}$, Fig. 2), ET-1 (0.1 nM - $0.1 \mu \mathrm{M}$, Fig. 3a-c) and $\mathrm{U} 46619$ (10 nM - $3 \mu \mathrm{M}$, Fig. 3d-e) contracted the MA rings in a concentrationdependent manner. As shown in Fig. $1 \mathrm{~b}$, the contractile efficacy $\left(E_{\max }\right)$ of $\mathrm{KCl}$ underwent a linear increase $(\mathrm{E} 15<\mathrm{E} 19<\mathrm{NH} 3 \mathrm{~h}<\mathrm{NH} 1 \mathrm{~d})$, whereas the potency $\left(p \mathrm{D}_{2}\right)$ did not change (Fig. 1C). The efficacy of NE (Fig. 2b) and U46619 (Fig. 3d) increased in ovo, but not ex ovo $(\mathrm{E} 15<\mathrm{E} 19=\mathrm{NH} 3 \mathrm{~h}=\mathrm{NH} 1 \mathrm{~d})$. The potency $\left(-\log \mathrm{ED}_{20}\right)$ of NE peaked in the $\mathrm{NH} 3 \mathrm{~h}$ and decreased at $\mathrm{NH} 1 \mathrm{~d}$ (Fig. 2c), whereas the contractile potency of $\mathrm{U} 46619$ (Fig. 3e) was not significantly different in the four groups of age. The efficacy of ET-1 (Fig. 3b) peaked at E19 (E15 < E19 > NH3h $=$ NH1d $)$ and the potency decreased with age (Fig. 3c). As shown in Fig. 3a, high concentrations of ET-1 (0.1 $\mu \mathrm{M})$ evoked a relaxant response in the rings from $\mathrm{NH}$ chicks. This relaxant effect of ET-1 was not observed at E15 or E19 (Fig. 3a). 

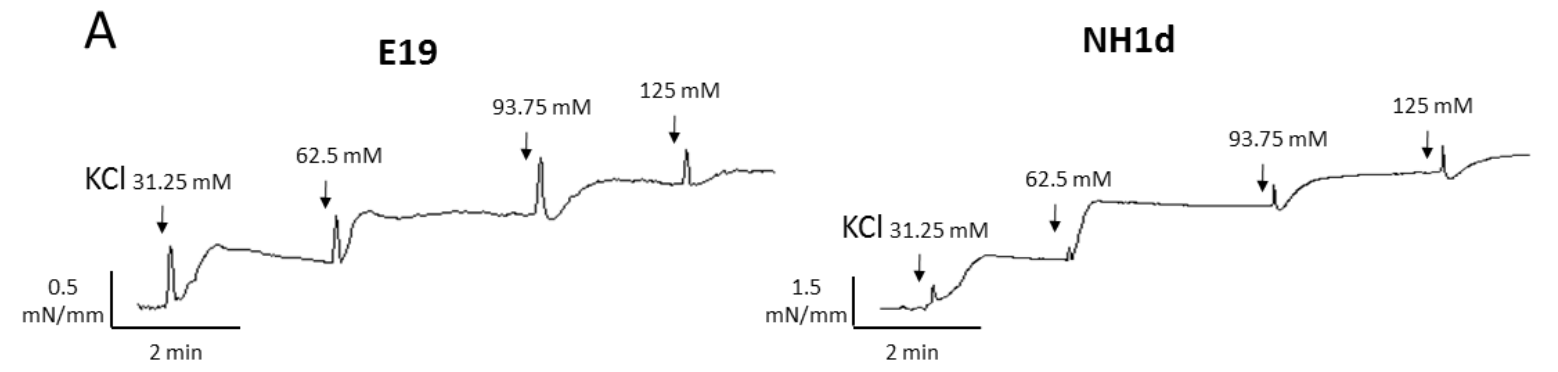

B

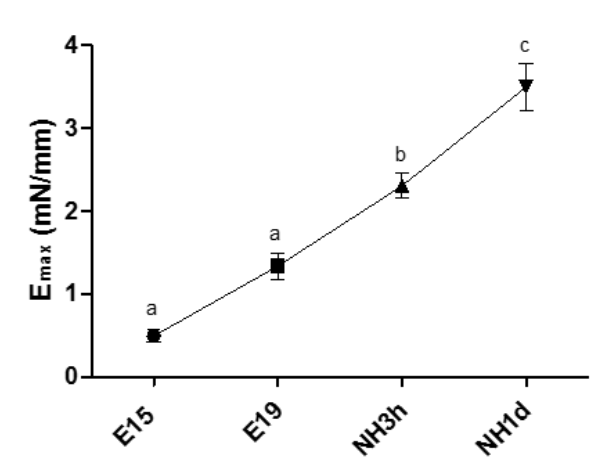

C

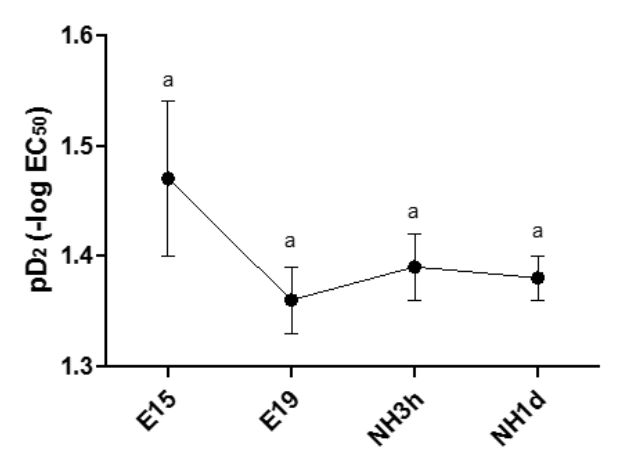

Figure 1. Contractile effects of $\mathrm{KCl}$ in mesenteric arteries from 15-day (E15) and 19-day (E19) chicken embryos and 3-h-old (NH3h) and 1-day-old (NH1d) newly hatched chicks. Values (mean \pm SEM) without a common letter are significantly different $(P<0.05)$. In $A$ (representative tracings), numbers above the arrows indicate [KCl]. 


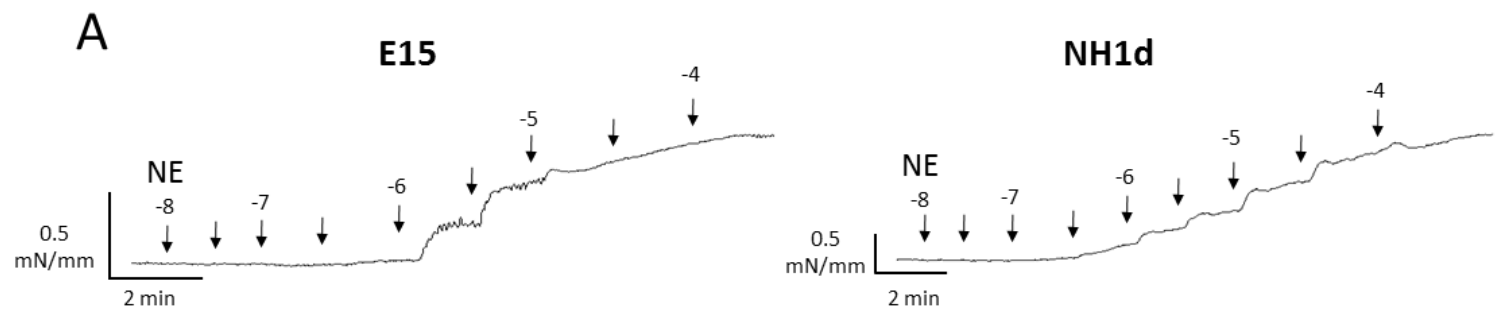

B

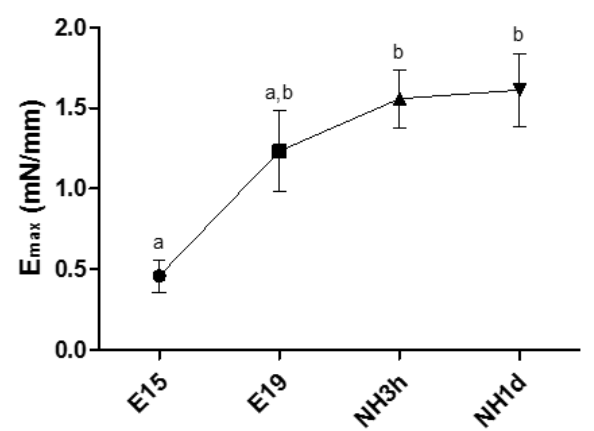

C

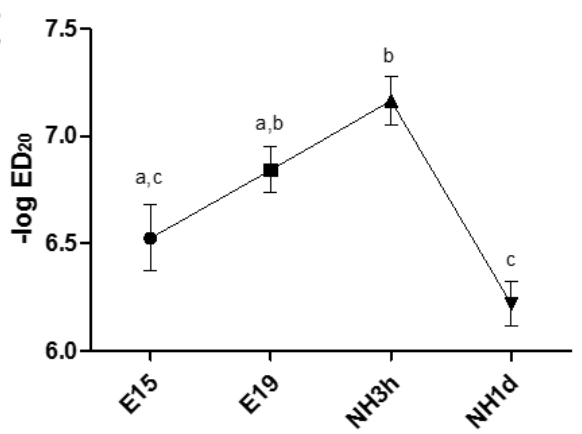

Figure 2. Contractile effects of norepinephrine (NE) in mesenteric arteries from 15-day (E15) and 19-day (E19) chicken embryos and 3-h-old (NH3h) and 1-day-old (NH1d) newly hatched chicks. Values (mean $\pm S E M)$ without a common letter are significantly different $(P<0.05)$. In $A$ (representative tracings), numbers above the arrows indicate log $M[N E]$ and arrows without numbers indicate half-log increments in concentration. 


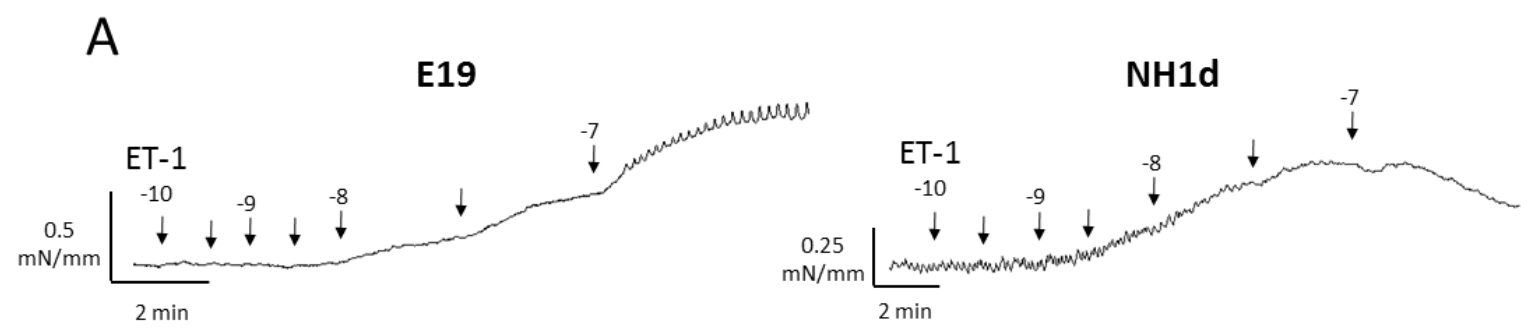

B

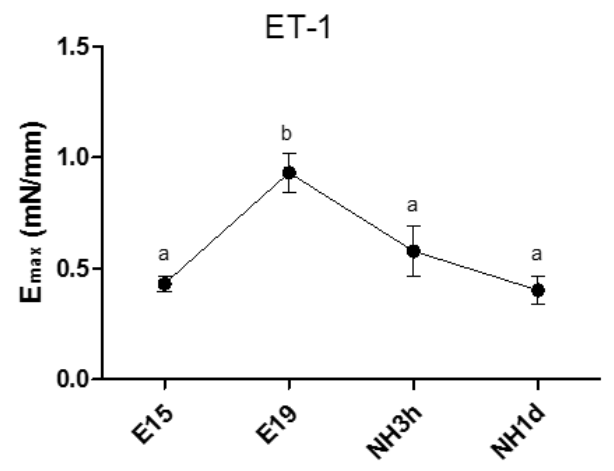

D

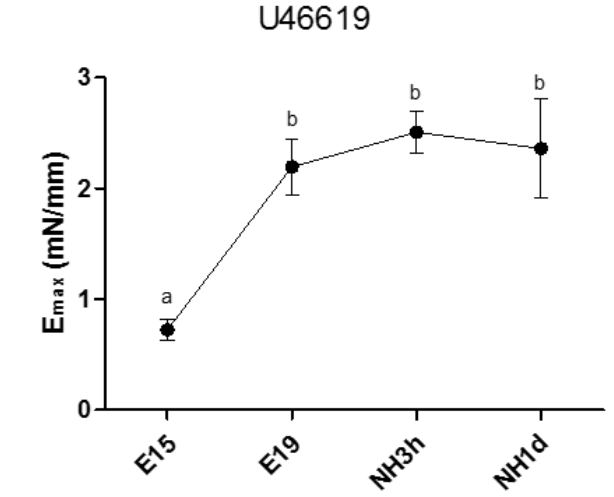

C

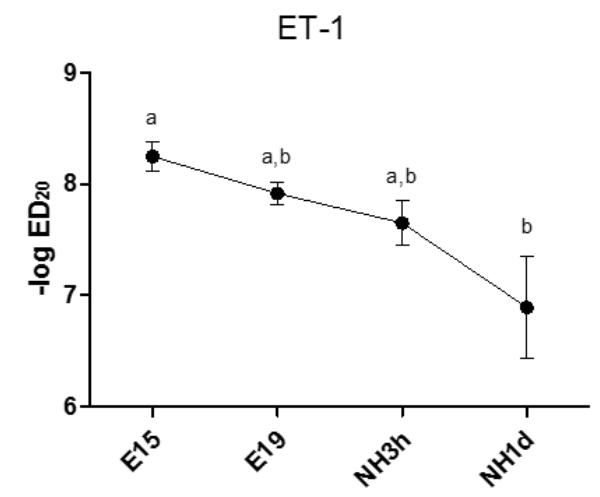

$E$

U46619

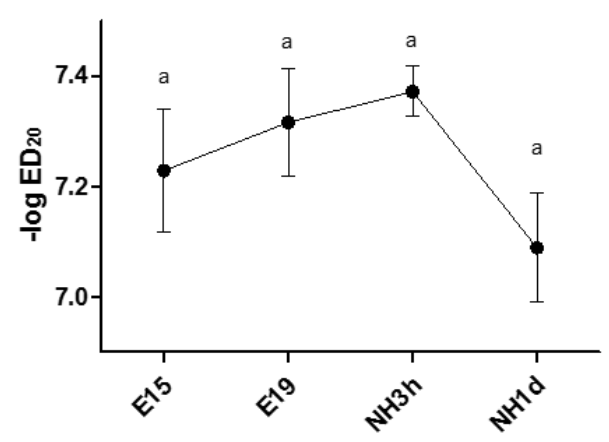

Figure 3. Contractile effects of endothelin-1 (ET-1, A-C) and the TP receptor agonist U46619 $(D, E)$ in mesenteric arteries from 15-day (E15) and 19-day (E19) chicken embryos and 3-hold (NH3h) and 1-day-old (NH1d) newly hatched chicks. Values (mean \pm SEM) without a common letter are significantly different $(P<0.05)$. In $A$ (representative tracings), numbers above the arrows indicate log $M$ [ET-1] and arrows without numbers indicate half-log increments in concentration. 


\section{RELAXANT RESPONSES}

As shown in Fig. 4, the muscarinic receptor agonist ACh (10 nM - $0.1 \mathrm{mM})$, the NO donor SNP (10 nM - $0.1 \mathrm{mM})$ and the adenylate cyclase activator forskolin (10 nM - 10 $\mu \mathrm{M})$ relaxed $\mathrm{NE}$ - and $\mathrm{KCl}$-contracted $\mathrm{MA}$ rings in a concentration-dependent manner. The response to ACh was abolished by mechanical removal of the endothelium whereas SNP- and forskolin-induced relaxations were not affected by this (data not shown). The relaxant efficacies $\left(E_{\max }\right)$ of ACh (Fig. 4b) and SNP (Fig. 4d) were significantly impaired in $\mathrm{KCl}$-contracted $\mathrm{MAs}$ when compared with $\mathrm{NE}$-contracted vessels. This effect of $\mathrm{KCl}$-contraction on relaxant efficacy was not observed for forskolin (with the exception of E15 MAs, Fig. 4f).

The relaxant efficacy of ACh did not change with age (Fig. 4b) but the potency was significantly lower in the E15 (Fig. 4c). As shown in Fig. 4b, the relaxant efficacy of ACh was impaired by the presence of the NO synthase inhibitor L-NAME. However this effect of L-NAME was only observed in the embryonic arteries (E15 and E19). On the other hand, L-NAME decreased the potency of ACh in the E15 and the NH1d arteries but not in the $\mathrm{E} 19$ or NH3h vessels (Fig. 4C).

The relaxant efficacy (Fig. 4d) and potency (Fig. 4e) of SNP underwent a slight, but significant decrease with age. Both SNP potency and efficacy were significantly impaired by the presence of the guanylate cyclase inhibitor ODQ. Finally, the relaxant efficacy of forskolin did not change with age (Fig. 4f) but the potency peaked in the $\mathrm{NH} 3 \mathrm{~h}$ arteries and significantly decreased afterwards $(\mathrm{E} 15=\mathrm{E} 19<\mathrm{NH} 3 \mathrm{~h}>\mathrm{NH} 1 \mathrm{~d}$, Fig. $4 g$ ). 
A E19 NH1d

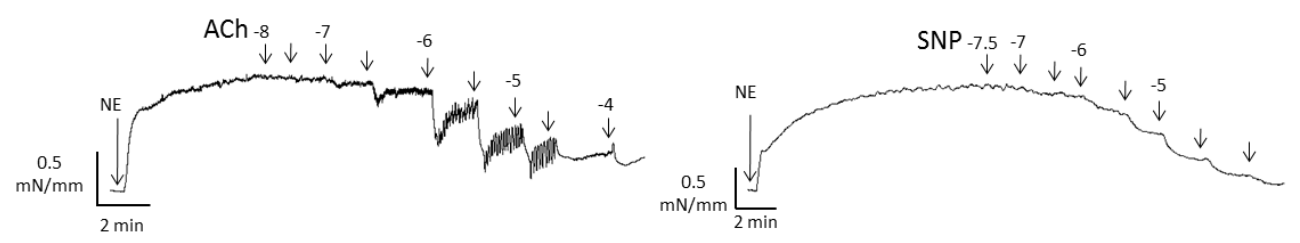

B

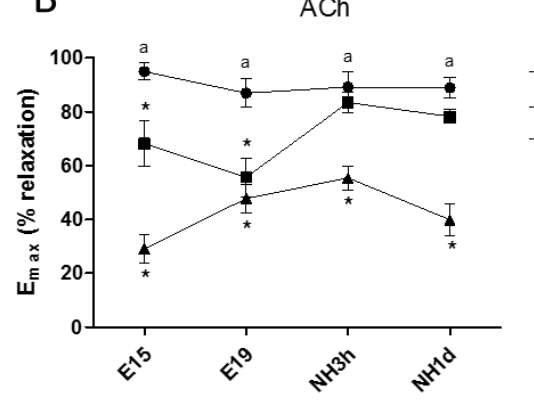

D

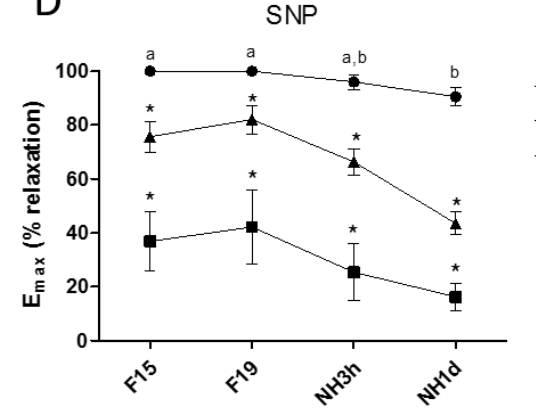

F

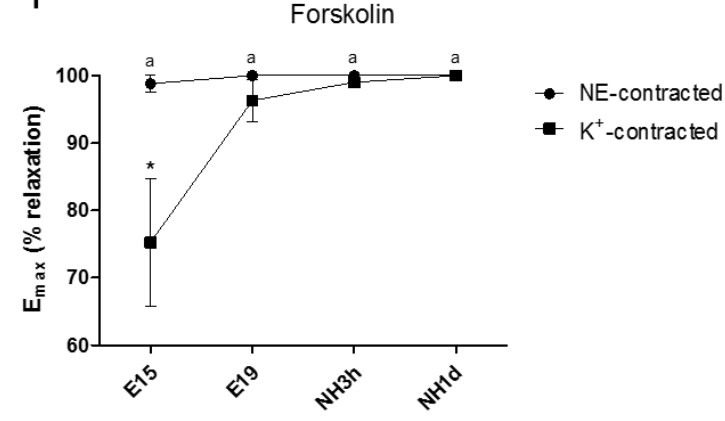

$\mathrm{C}$

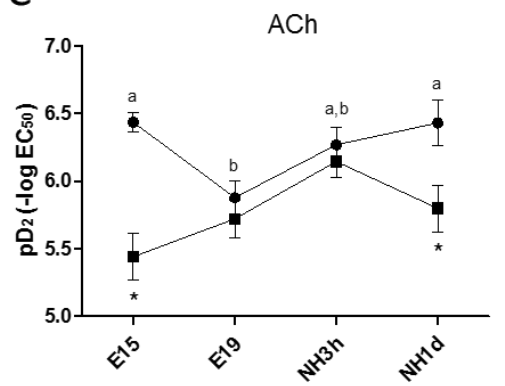

$E$

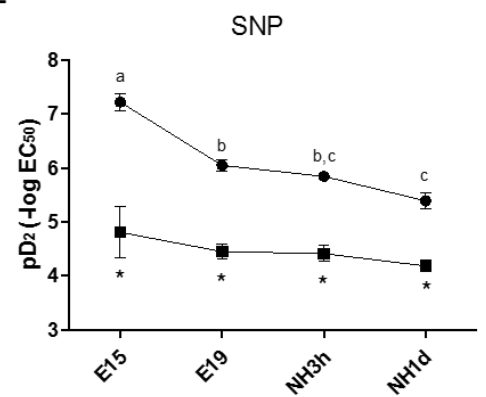

G

Forskolin

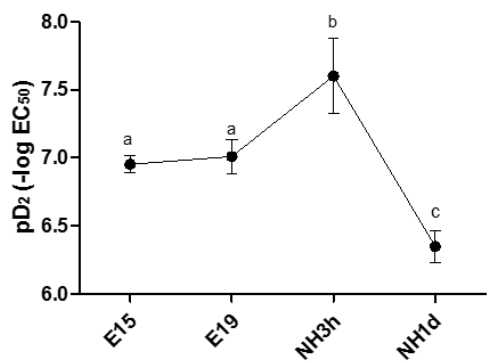

Figure 4. Relaxant effects of acetylcholine (ACh, $A$ left and $B, C)$, sodium nitroprusside (SNP, $A$ right and $D, E)$ and forskolin $(F, G)$ in mesenteric arteries from 15-day (E15) and 19-day (E19) chicken embryos and 3-h-old (NH3h) and 1-day-old (NH1d) newly hatched chicks. The vessels were contracted with $\mathrm{KCl}(62.5 \mathrm{mM})$ or norepinephrine $(10 \mu \mathrm{M})$. The effects of the NO synthase inhibitor L-NAME $(0.1 \mathrm{mM}, B)$ and the soluble guanylate cyclase ODQ $(10 \mu M, D)$ on ACh- and SNP-evoked relaxation are also shown. Developmental changes: values (mean \pm SEM) without a common letter are significantly different $(P<0.05)$. ${ }^{*} P<0.05$ versus $N E$ contracted (same age). In A (representative tracings), numbers above the arrows indicate log $M$ [drug] and arrows without numbers indicate half-log increments in concentration. 


\section{DISCUSSION}

During prenatal life, endothelial and vascular smooth muscle cells play a key role in blood vessel morphogenesis and exhibit high rates of proliferation, migration, and production of extracellular matrix. ${ }^{32,33}$ These processes occur while the new forming vessels are simultaneously acquiring the capacity to regulate vascular tone and undergoing developmental changes in the contractile apparatus, the density of receptors and the signal transduction pathways leading to contraction/relaxation. ${ }^{34}$ This results in developmental changes in vascular reactivity. In this study, we analyzed the maturational differences in the reactivity of MAs isolated from chicken embryos and hatchlings. We found that MAs from 15-day chick embryos responded to $\mathrm{K}^{+}$- evoked depolarization and to a variety of agonists (NE, ET-1, ACh, SNP, forskolin) indicating the presence at this stage of development of electro- and pharmaco-mechanical coupling as well as CGMP- and CAMP-mediated relaxation. In ovo development and transition to ex-ovo life was accompanied by alterations in the response of the MAs but a different developmental trajectory was observed for each reactivity pathway tested. Thus, the efficacy of $\mathrm{K}^{+}$to contract MAs underwent a linear developmental increase $(\mathrm{E} 15<\mathrm{E} 19<\mathrm{NH} 3 \mathrm{~h}<\mathrm{NH} 1 \mathrm{~d})$, whereas the efficacy of $\mathrm{NE}$ and $\mathrm{U} 46619$ increased in ovo but not ex ovo ( $\mathrm{E} 15<\mathrm{E} 19=\mathrm{NH} 3 \mathrm{~h}=\mathrm{NH} 1 \mathrm{~d})$, and the efficacy of $\mathrm{ET}$ 1 reached a peak in the $\mathrm{E} 19$ chicken $(\mathrm{E} 15<\mathrm{E} 19>\mathrm{NH} 3 \mathrm{~h}=\mathrm{NH} 1 \mathrm{~d})$. The relaxations elicited by ACh, SNP, and forskolin did not undergo dramatic developmental changes, suggesting that maturation of vasodilator mechanisms precedes that of vasoconstrictor mechanisms.

\section{RESPONSE OF MESENTERIC ARTERIES TO CONTRACTILE STIMULI}

$\mathrm{KCl}$ has long been used as a tool to bypass $\mathrm{G}$ protein-coupled receptors and activate smooth muscle by a highly reproducible receptor-independent mechanism involving depolarization, activation of voltage-operated $\mathrm{Ca}^{2+}$ channels, increase in cytosolic free $\mathrm{Ca}^{2+}$, $\mathrm{Ca}^{2+}$-dependent myosin light chain phosphorylation and contraction. ${ }^{35}$ Although recent data indicate that $\mathrm{KCl}$ also can regulate the degree of $\mathrm{Ca}^{2+}$ sensitivity in smooth muscle, $\mathrm{KCl}$-induced contraction is often used to examine the smooth muscle contraction function and to standardize the receptor-mediated contraction. ${ }^{35}$ In the present work, we observed that $\mathrm{KCl}$-evoked depolarization induced a significant 
contraction in the MA of the 15-day embryos, indicating the presence of a relatively mature contractile apparatus. Interestingly, the magnitude of $\mathrm{KCl}$-induced contraction in MA increased two and half-fold between days 15 and 19, whereas in chicken embryo carotid and femoral arteries $\mathrm{KCl}$-evoked contraction increased five- to sevenfold during the same incubation period (Fig. 5). ${ }^{36}$ Sensitivity to $\mathrm{KCl}$ was unaltered over the period we examined. The developmental differences in $\mathrm{KCl}$-induced contraction could reflect an increase in vascular smooth muscle mass or dissimilarities in vascular smooth muscle phenotype. The expression of smooth muscle proteins and myosin heavy chain isoforms have been shown to be developmentally regulated and tissue dependent in mammals. ${ }^{37,38}$ From the different chicken embryo vessels that our group have studied -carotid, femoral, pulmonary, chorioallantoic (CA) arteries, and ductus arteriosus (DA)only the $\mathrm{DA}^{15,39,40}$ and the $\mathrm{CA}$ arterie ${ }^{30}$ showed an early "mature" contractile function at E15 comparable to the one observed in the MA (Fig. 5). This high degree of responsiveness of the E15 MA suggests the presence of an active control of mesenteric circulation at this stage of development. 
mesenteric ductus art. chorioallantoic pulmonary femoral carotid
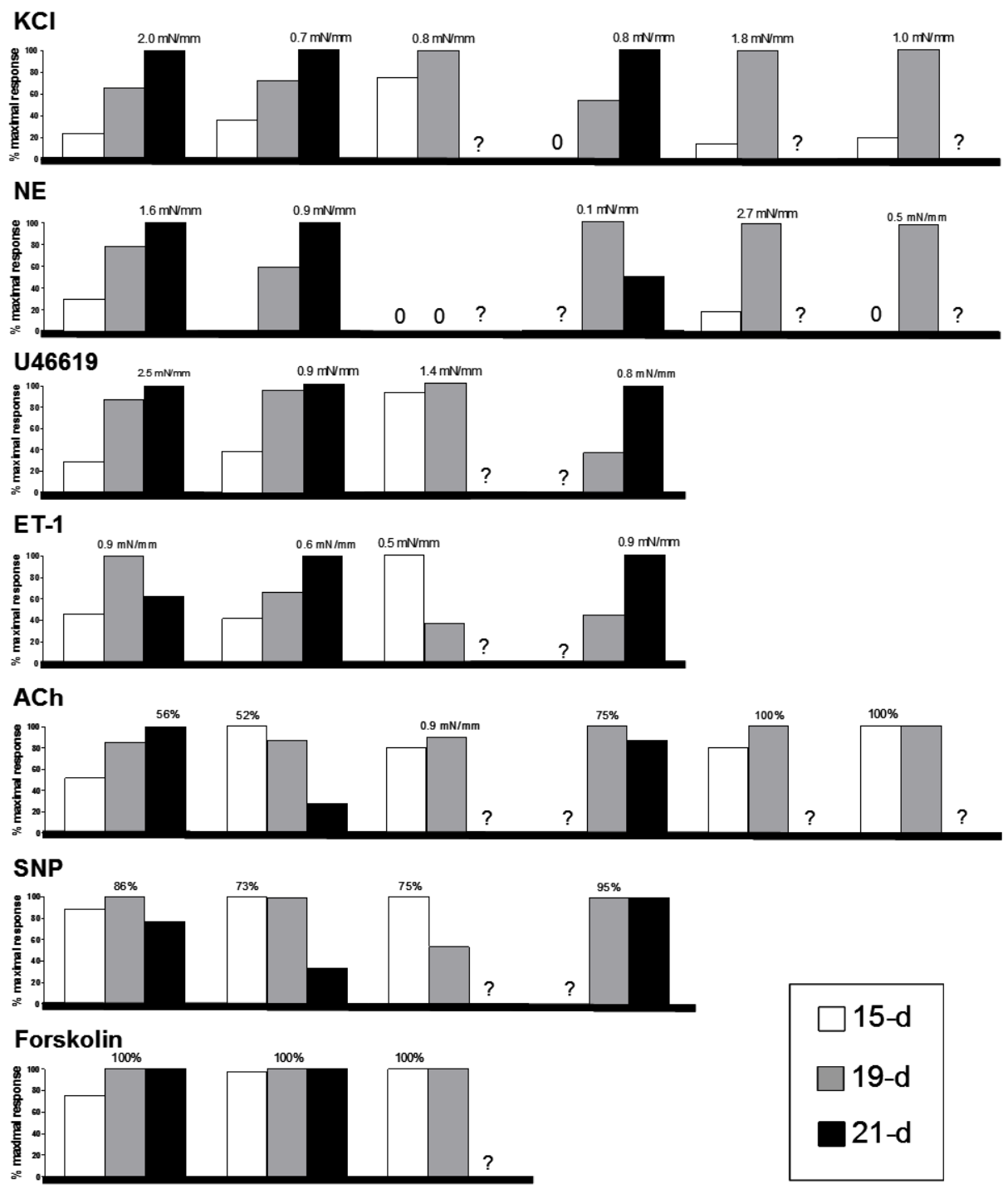

$15-\mathrm{d}$

19-d

$21-\mathrm{d}$

Figure 5. Overview of the developmental changes in vascular reactivity of the chicken embryo. The figure has been constructed with data from the present work (mesenteric artery); Agren et al., 2007; 2008 and 2009 (ductus arteriosus) 13,14,39; Lindgren et al., 2010 (chorioallantoic artery) ${ }^{30}$; Villamor et al., 2002 (pulmonary artery) ${ }^{17}$; and le Noble et al., 2000 (femoral and carotid arteries) ${ }^{36}$. The 21-day group is formed by newly hatched chicks (mesenteric arteries) or externally pipped embryos (rest of the vessels). The data are presented as percent of the maximal response observed among the three ages. This maximal response is indicated above the corresponding column (as $\mathrm{mN} / \mathrm{mm}$ for the contractions and as percent relaxation for the relaxations). The "?" symbol means unknown data and the "0" means no response. For the study of the relaxant responses the vessels were pre-contracted with $\mathrm{KCl}$. Note that ACh did not evoke relaxation but contraction in the chorioallantoic arteries. NE norepinephrine, U46619

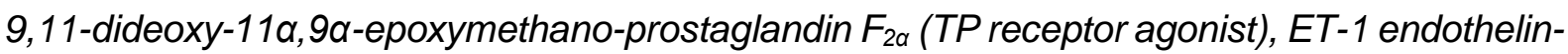
1, ACh acetylcholine, SNP sodium nitroprusside. 
Catecholamines play a key role in the prenatal cardiovascular response to stress and in several of the adaptations that characterize the transition from the pre- to the neonatal period. ${ }^{41-43}$ In the mammalian fetus, the cardiovascular responses to acute hypoxia include a redistribution of the cardiac output away from the periphery towards high priority organs such as the heart, brain, and adrenal gland. ${ }^{44}$ Similarly, in the chicken embryo acute hypoxia caused a redistribution of the cardiac output in favor of heart, brain and CA membrane at the expense of intestine, yolk-sac, liver, and carcass. ${ }^{45} \mathrm{~A}$ component of the this protective redistribution is mediated by increased release of catecholamines ${ }^{41-43}$, whose effects would be modulated by the different responsiveness of the vascular beds. Accordingly, we show herein that NE induced a significant contraction in the E15 MA and, in a previous study, Rouwet et al. demonstrated that $\mathrm{NE}$-induced vasoconstriction was already present in second-order MAs of 13-day chicken embryos. ${ }^{16}$ They also observed a developmental increase in the MA responsiveness to NE similar to the described in the present study. Interestingly, the development of adrenergic contraction in the chicken carotid artery ${ }^{36}$ and $\mathrm{DA}^{39}$ is slower than the observed in the MA (Fig. 5). Moreover, the chicken CA arteries did not show adrenergic contraction at any stage of development. ${ }^{30}$ Taken all together, these results show a correlation between the distribution of cardiac output during hypoxia (i.e. increased to the brain and the CA membrane and decreased to the intestine) and the local vascular responses to adrenergic stimulation.

ET-1 is a vasoactive and mitogenic polypeptide produced mainly by the vascular endothelium. Its binding to $E T_{A}$ and $E T_{B}$ receptors on vascular smooth muscle induces contraction, whereas its binding to endothelial $E T_{B}$ receptors causes vasodilatation. ${ }^{3,46}$ ET-1 is constitutively produced, but its production can also be stimulated by a wide range of stimuli, including decreased flow, hypoxia, and various inflammatory cytokines. In some mammalian species, ET-1 is considered as the primary vasoconstrictor stimulus in the perinatal intestinal circulation. 3,5,47 In the present work we show the responsiveness of embryonic and newly hatched chicken MA to ET-1. Previously we demonstrated ET-1-induced contraction in chicken embryo femoral, pulmonary, and CA arteries as well as in the DA (Fig. 5). Interestingly, ET-1-induced contraction reached a peak in the E19 and decreased in the $\mathrm{NH}$ chicken. Moreover, high concentrations of ET-1 induced relaxation in the MA from $\mathrm{NH}$ chickens. Although, 
we have not characterized the nature of the response to ET-1, our result suggest the

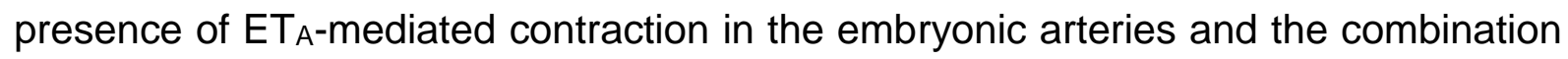
of $E T_{A}$-mediated contraction and $E T_{B}$-mediated relaxation in the $M A$ from the $\mathrm{NH}$ chickens. Endothelial ETB receptors are abundantly present in 1- to 3-day-old swine intestine and their activation leads to a modest NO-dependent vasodilatation. However, the force of $E T_{A}$-induced vasoconstriction exceeds that of $E T_{B}$-generated vasodilatation, such that the net effect of the constitutive production of ET-1 in the swine neonatal intestine resistance vessels is vasoconstriction. ${ }^{3,5,47}$ The present findings in the chicken MA warrant further investigation into the constitutive production of ET-1 and the developmental changes in ET receptor density.

The TP receptor agonist U46619 evoked contraction of chicken embryo mesenteric (present work), pulmonary ${ }^{17}$, femoral ${ }^{18,19}, \mathrm{CA}^{30}$ arteries and DA ${ }^{39,48}$ (Fig. 5). Sensitivity of the MA to U46619 was unaltered over the period we examined, but the magnitude of U46619-induced contraction increased threefold between E15 and E19. Accordingly, U46619-evoked contraction of fetal lamb MAs increased with gestational age. ${ }^{8}$ In the first hours of ex ovo life, the responsiveness of chicken MA to U46619 remained unchanged and our study was not extended beyond that time point. However, a postnatal decrease in MA responsiveness to U46619 and other TP receptor agonists (such as isoprostanes) has been characterized in swine ${ }^{49}$, suggesting that TP receptor agonists may play a role in the postnatal reduction of mesenteric vascular resistance.

\section{RELAXANT RESPONSES}

ACh has been widely used in numerous vascular beds to stimulate endotheliumdependent relaxation. ${ }^{50,51}$ Several endothelium-derived relaxing factors have been found, including NO, prostaglandins, carbon monoxide and a yet unidentified factor called endothelium-derived hyperpolarizing factor ${ }^{52,53}$ (EDHF). The contribution of each of these factors to endothelium-dependent relaxation varies across vascular beds and also with the physiological or pharmacological stimuli used to stimulate the endothelium. ${ }^{53}$ In the chicken embryo, ACh induced an endothelium-dependent and, at least partially, NO-mediated relaxation of the $\mathrm{DA}^{39,48}$, the pulmonary ${ }^{17}$, the femoral $^{17}$, and the carotid arteries ${ }^{36}$ (Fig. 5). In the present work, we show that ACh 
evoked endothelium-dependent relaxation in MA from embryonic and $\mathrm{NH}$ chickens. Rouwet et al. ${ }^{16}$ showed that ACh-induced vasodilatation was already present in E13 MAs. Herein, we observed that the efficacy and potency of ACh in relaxing MAs did not change with development. However, the NO synthase inhibitor L-NAME decreased the efficacy $\left(E_{\max }\right)$ of $\mathrm{ACh}$ in embryonic, but not in $\mathrm{NH}$ arteries and decreased the potency $\left(p D_{2}\right)$ of $A C h$ only in the $E 15$ and the $1 \mathrm{dNH}$ vessels, suggesting developmental differences in the underlying mechanism(s). Thus, our results suggest a more relevant role of NO in the endothelium-dependent actions of ACh in the MAs from the less mature animals.

The identity of EDHF remains uncertain, but it is accepted that its action involves an increase in $\mathrm{K}^{+}$conductance and can, therefore, be inhibited by abolishing the electrochemical gradient for $\mathrm{K}^{+}$ions. This was conducted in these studies by using a solution containing $62.5 \mathrm{mM} \mathrm{K}^{+}$to induce tone. Under these experimental conditions, it was observed a significant impairment of ACh-induced relaxation. This impairment was similar in the four age groups and may suggest a relevant role for EDHF in perinatal chicken MAs. However, we observed that SNP was also less efficacious in $\mathrm{KCl}$ - than in NE-contracted arteries indicating that sGC-dependent relaxation is also affected by depolarization in this preparation. Interestingly, the relaxant efficacy of the adenylate cyclase stimulator forskolin was impaired by high $\mathrm{K}^{+}$depolarizing solution only in the youngest vessels. Again, the maturational changes in MA reactivity appear to affect not only the potency/efficacy of the vasoactive agonists, but also alterations in the underlying pathways.

\section{SIGNIFICANCE AND PERSPECTIVES}

The control of intestinal vascular resistance during the perinatal period is a complex process that integrates the intestinal circulation into cardiovascular reflexes which preserve systemic homeostasis, often at the expense of the local intestinal circulation. ${ }^{54,55}$ As reviewed by Nankervis et al. ${ }^{5}$, the mammalian intestinal vascular tone is maintained by a dynamic balance between constrictor (ET-1) and dilator (NO) stimuli. In the newborn, under steady state conditions, the balance favors vasodilatation generating an increased rate of blood flow to the newborn intestine to meet the metabolic demands of early postnatal life. Our data suggest the presence of 
similar regulatory mechanisms in the chicken mesenteric circulation. Understanding the basic mechanisms of either normal or altered functional and structural development of the mesenteric vessels, as well as inter-species differences in mesenteric circulation, may provide insights into human and animal intestinal disease. 


\section{REFERENCES}

1 Edelstone, D. I. \& Holzman, I. R. Fetal intestinal oxygen consumption at various levels of oxygenation. Am J Physiol 242, H50-54 (1982).

2 Nankervis, C. A., Reber, K. M. \& Nowicki, P. T. Age-dependent changes in the postnatal intestinal microcirculation. Microcirculation 8, 377-387 (2001).

3 Reber, K. M., Nankervis, C. A. \& Nowicki, P. T. Newborn intestinal circulation. Physiology and pathophysiology. Clin Perinatol 29, 23-39 (2002).

4 Heymann, M. A., Iwamoto, H. S. \& Rudolph, A. M. Factors affecting changes in the neonatal systemic circulation. Annu Rev Physiol 43, 371-383 (1981).

5 Nankervis, C. A., Giannone, P. J. \& Reber, K. M. The neonatal intestinal vasculature: contributing factors to necrotizing enterocolitis. Semin Perinato/32, 83-91 (2008).

6 Moonen, R. M. et al. Carbamoyl phosphate synthetase polymorphisms as a risk factor for necrotizing enterocolitis. Pediatr Res 62, 188-190 (2007).

7 Moonen, R. M. et al. The T1405N carbamoyl phosphate synthetase polymorphism does not affect plasma arginine concentrations in preterm infants. PLoS One 5, e10792 (2010).

8 Wolfs, T. G. et al. Endotoxin induced chorioamnionitis prevents intestinal development during gestation in fetal sheep. PLoS One 4, e5837 (2009).

9 Lin, P. W., Nasr, T. R. \& Stoll, B. J. Necrotizing enterocolitis: recent scientific advances in pathophysiology and prevention. Semin Perinato/32, 70-82 (2008).

10 Ruijtenbeek, K., De Mey, J. G. \& Blanco, C. E. The chicken embryo in developmental physiology of the cardiovascular system: a traditional model with new possibilities. Am J Physiol Regul Integr Comp Physiol 283, R549-550; author reply R550-541 (2002).

11 Sutendra, G. \& Michelakis, E. D. The chicken embryo as a model for ductus arteriosus developmental biology: cracking into new territory. Am J Physiol Regul Integr Comp Physiol 292, R481-484 (2007).

12 Agren, P. et al. Ontogeny of chicken ductus arteriosus response to oxygen and vasoconstrictors. American journal of physiology Regulatory, integrative and comparative physiology 292, R485-496 (2007). 
13 Agren, P., van der Sterren, S., Cogolludo, A. L., Blanco, C. E. \& Villamor, E. Developmental changes in the effects of prostaglandin E2 in the chicken ductus arteriosus. J Comp Physiol [B] 179, 133-143 (2009).

14 Agren, P. et al. Developmental changes in endothelium-dependent relaxation of the chicken ductus arteriosus. J Physiol Pharmacol 59, 55-76 (2008).

15 Moonen, R. M. J., Agren, P., Cogolludo, A. L., Perez-Vizcaino, F. \& Villamor, E. Response of Chicken Ductus Arteriosus to Hypercarbic and Normocarbic Acidosis. Neonatology 98, 47-56 (2010).

16 Rouwet, E. V. et al. Development of vasomotor responses in fetal mesenteric arteries. Am J Physiol Heart Circ Physiol 279, H1097-1105 (2000).

17 Villamor, E., Ruijtenbeek, K., Pulgar, V., De Mey, J. G. \& Blanco, C. E. Vascular reactivity in intrapulmonary arteries of chicken embryos during transition to ex ovo life. Am J Physiol Regul Integr Comp Physiol 282, R917-927 (2002).

18 Zoer, B., Blanco, C. E. \& Villamor, E. Role of Rho-kinase in mediating contraction of chicken embryo femoral arteries. J Comp Physiol B 180, 427-435 (2010).

19 Zoer, B. et al. Hypoxia sensing in the fetal chicken femoral artery is mediated by the mitochondrial electron transport chain. Am J Physiol Regul Integr Comp Physiol 298, R1026-1034 (2010).

20 Zoer, B. et al. Effects of prenatal hypoxia on pulmonary vascular reactivity in chickens prone to pulmonary hypertension. J Physiol Pharmacol 60, 119-130 (2009).

21 Speake, B. K., Murray, A. M. \& Noble, R. C. Transport and transformations of yolk lipids during development of the avian embryo. Prog Lipid Res 37, 1-32 (1998).

22 Powell, K. A., Deans, E. A. \& Speake, B. K. Fatty acid esterification in the yolk sac membrane of the avian embryo. J Comp Physiol B 174, 163-168 (2004).

23 Noy, Y. \& Sklan, D. Yolk and exogenous feed utilization in the posthatch chick. Poult Sci 80, 1490-1495 (2001).

24 Sklan, D. Fat and carbohydrate use in posthatch chicks. Poult Sci 82, 117-122 (2003). 
25 Uni, Z., Tako, E., Gal-Garber, O. \& Sklan, D. Morphological, molecular, and functional changes in the chicken small intestine of the late-term embryo. Poult Sci 82, 1747-1754 (2003).

26 Noy, Y. \& Sklan, D. Yolk utilisation in the newly hatched poult. Br Poult Sci 39, 446-451 (1998).

27 Biviano, A. B., Martinez del Rio, C. \& Phillips, D. L. Ontogenesis of intestine morphology and intestinal disaccharidases in chickens (Gallus gallus) fed contrasting purified diets. J Comp Physiol B 163, 508-518 (1993).

28 Levinsohn, E. M., Packard, D. S., Jr., West, E. M. \& Hootnick, D. R. Arterial anatomy of chicken embryo and hatchling. Am J Anat 169, 377-405 (1984).

29 Altimiras, J. \& Crossley, D. A., 2nd. Control of blood pressure mediated by baroreflex changes of heart rate in the chicken embryo (Gallus gallus). Am J Physiol Regul Integr Comp Physiol 278, R980-986 (2000).

30 Lindgren, I., Zoer, B., Altimiras, J. \& Villamor, E. Reactivity of chicken chorioallantoic arteries, avian homologue of human fetoplacental arteries J Physiol Pharmacol (2010).

31 Gluais, P., Lonchampt, M., Morrow, J. D., Vanhoutte, P. M. \& Feletou, M. Acetylcholine-induced endothelium-dependent contractions in the SHR aorta: the Janus face of prostacyclin. Br J Pharmacol 146, 834-845 (2005).

32 Owens, G. K., Kumar, M. S. \& Wamhoff, B. R. Vol. 84 767-801 (2004).

33 Rzucidlo, E. M., Martin, K. A. \& Powell, R. J. Regulation of vascular smooth muscle cell differentiation. J Vasc Surg 45 Suppl A, A25-32 (2007).

34 Altimiras, J., Crossley, D. A. \& Villamor, E. in Cardio-Respiratory Control in Vertebrates. Comparative and Evolutionary Aspects Vol. 3 (eds M.L. Glass \& S.C. Wood) 397-427 (Springer-Verlag 2009).

35 Ratz, P. H., Berg, K. M., Urban, N. H. \& Miner, A. S. Regulation of smooth muscle calcium sensitivity: $\mathrm{KCl}$ as a calcium-sensitizing stimulus. Am J Physiol Cell Physiol 288, C769-783 (2005).

36 le Noble, F. A., Ruijtenbeek, K., Gommers, S., de Mey, J. G. \& Blanco, C. E. Contractile and relaxing reactivity in carotid and femoral arteries of chicken embryos. Am J Physiol Heart Circ Physiol 278, H1261-1268 (2000). 
37 Sakurai, H. et al. Expression of four myosin heavy chain genes in developing blood vessels and other smooth muscle organs in rabbits. Eur J Cell Biol 69, 166-172 (1996).

38 Slomp, J. et al. Differentiation, dedifferentiation, and apoptosis of smooth muscle cells during the development of the human ductus arteriosus. Arterioscler Thromb Vasc Biol 17, 1003-1009 (1997).

39 Agren, P. et al. Ontogeny of chicken ductus arteriosus response to oxygen and vasoconstrictors. Am J Physiol Regul Integr Comp Physiol 292, R485-496 (2007).

40 Flinsenberg, T. W. et al. Effects of gender and estrogen on chicken ductus arteriosus reactivity. Am J Physiol Regul Integr Comp Physiol. 298, R1217-1224 (2010).

41 Mulder, A. L., Golde, J. M., Goor, A. A., Giussani, D. A. \& Blanco, C. E. Developmental changes in plasma catecholamine concentrations during normoxia and acute hypoxia in the chick embryo. J Physiol 527 Pt 3, 593-599 (2000).

42 Mulder, A. L., Miedema, A., De Mey, J. G., Giussani, D. A. \& Blanco, C. E. Sympathetic control of the cardiovascular response to acute hypoxemia in the chick embryo. Am J Physiol Regul Integr Comp Physiol 282, R1156-1163 (2002).

43 Mulder, A. L., van Goor, C. A., Giussani, D. A. \& Blanco, C. E. Alpha-adrenergic contribution to the cardiovascular response to acute hypoxemia in the chick embryo. Am J Physiol Regul Integr Comp Physiol 281, R2004-2010 (2001).

44 Llanos, A. J. et al. The fetal llama versus the fetal sheep: different strategies to withstand hypoxia. High Alt Med Biol 4, 193-202 (2003).

45 Mulder, A. L., van Golde, J. C., Prinzen, F. W. \& Blanco, C. E. Cardiac output distribution in response to hypoxia in the chick embryo in the second half of the incubation time. J Physiol 508 ( Pt 1), 281-287 (1998).

46 Granger, J. P. Endothelin. Am J Physiol Regul Integr Comp Physiol 285, R298301 (2003).

47 Nankervis, C. A. \& Nowicki, P. T. Role of endothelin-1 in regulation of the postnatal intestinal circulation. Am J Physiol Gastrointest Liver Physiol 278, G367-375 (2000). 
48 Schuurman, M. J. \& Villamor, E. Endothelium-dependent contraction induced by acetylcholine in the chicken ductus arteriosus involves cyclooxygenase- 1 activation and TP receptor stimulation. Comp Biochem Physiol A Mol Integr Physiol 157, 28-34 (2010).

49 Gonzalez-Luis, G. et al. Age-related differences in vasoconstrictor responses to isoprostanes in piglet pulmonary and mesenteric vascular smooth muscle. Pediatr Res 57, 845-852 (2005).

50 González-Luis, G. et al. Nitric oxide-mediated nonadrenergic noncholinergic relaxation of piglet pulmonary arteries decreases with postnatal age. Journal of Physiology and Pharmacology 58, 45-56 (2007).

51 Ignarro, L. J. Nitric oxide as a unique signaling molecule in the vascular system: a historical overview. J Physiol Pharmacol 53, 503-514 (2002).

52 Baragatti, B. et al. Interactions between NO, CO and an endothelium-derived hyperpolarizing factor (EDHF) in maintaining patency of the ductus arteriosus in the mouse. Br J Pharmacol 151, 54-62 (2007).

53 Busse, R. et al. EDHF: bringing the concepts together. Trends Pharmacol Sci 23, 374-380 (2002).

54 Duckles, S. P. \& Banner, W., Jr. Changes in vascular smooth muscle reactivity during development. Annu Rev Pharmacol Toxicol 24, 65-83 (1984).

55 Nowicki, P. Intestinal ischemia and necrotizing enterocolitis. J Pediatr 117, S1419 (1990) 
Chapter 3 MESENTERIC ARTERY REACTIVITY AND SMALL INTESTINE MORPHOLOGY IN A CHICKEN MODEL OF HYPOXIA-INDUCED FETAL GROWTH RESTRICTION

\author{
Rob M.J. Moonen, Carolina G.A. Kessels, Luc J.I. Zimmermann \\ and Eduardo Villamor
}

J Physiol Pharmacol 63, 601-612 (2012)

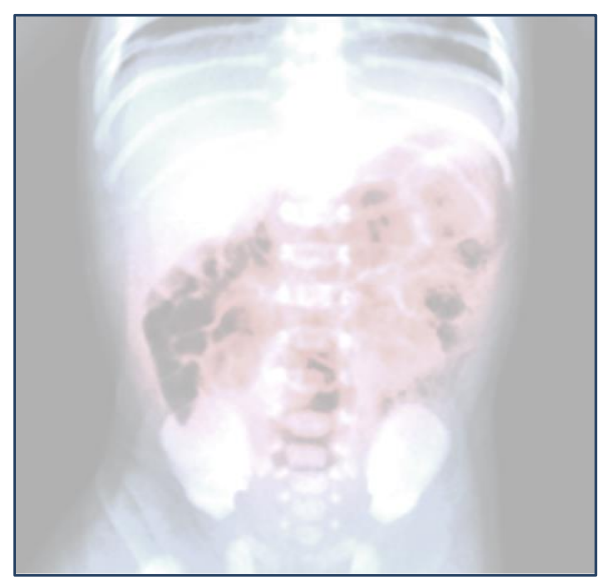




\section{ABSTRACT}

Infants with intrauterine growth retardation are prone to intestinal disorders. The morphological and molecular mechanisms that lead to these complications are not completely understood and suitable experimental models are necessary. The aim of this study was to characterize mesenteric artery (MA) reactivity, small intestine morphometry and intestinal expression of vascular endothelial growth factor (VEGF) in a chicken model of hypoxia-induced fetal growth restriction. Chicken embryos (15 and 19 incubation days) and hatchlings (<3-h-old and 1-day-old) were incubated under hypoxic ( $15 \%$ O2 from day 0 to day 19 of incubation) or normoxic conditions. Vascular reactivity was studied using wire miography. Intestinal morphometry was assessed in hematoxyline-eosine-stained sections. VEGF mRNA expression was determined by RT-PCR analysis. Hypoxia increased the responsiveness of chicken embryo MAs to the adrenergic agonist norepinephrine, the polypeptide endothelin (ET)-1, and the nitric oxide donor sodium nitroprusside and decreased the responsiveness to the endothelium-dependent relaxant agonist acetylcholine. However, the majority of these alterations, with the exception of the hyperresponsiveness to ET-1, were not present in the hypoxic hatchlings. When intestinal histology was analyzed, subtle hypoxiainduced changes were noted in the villi and the muscularis propria from the hatchlings. Hypoxic incubation also diminished the expression of VEGF mRNA in the terminal ileum of the hatchlings. In conclusion, chronic moderate hypoxia during incubation results in subtle but significant alterations in chicken MA reactivity, small intestine morphology and VEGF expression. Whether these alterations may have a direct effect on the functional status of the intestine remains to be investigated. 


\section{INTRODUCTION}

Intrauterine growth retardation (IUGR) is most frequently the consequence of placental insufficiency resulting in decreased availability of nutrients and oxygen. ${ }^{1}$ Infants with IUGR are prone to intestinal dysfunction which is manifested by feeding intolerance, poor growth, malabsorption, and, in the most severe cases, necrotizing enterocolitis (NEC). ${ }^{1,2}$ Moreover, it has been suggested that some of the intestinal alterations induced by IUGR may persist in later life. ${ }^{3}$ The morphological and molecular mechanisms that lead to these complications are not completely understood and suitable experimental models are necessary. In addition, it is difficult to demonstrate or isolate the effects of hypoxia because all human conditions or experimental animal models that induce fetal hypoxia are also accompanied by changes in nutrient delivery. ${ }^{4}$ In the last years, the chicken embryo has emerged as a valuable model for the study of the developmental consequences of hypoxia and other prenatal insults. . $^{5-}$ ${ }^{11}$ Hypoxia is easily induced by incubating the egg in a low oxygen environment and its effects can be studied without interferences of maternal hormonal, metabolic, or hemodynamic alterations.

Hypoxia has profound effects on endothelial and vascular smooth muscle cellular physiology affecting the transcriptionally regulated expression of vasoactive substances, the modulation of receptor populations, the density and activities of ion channels and the signal transduction pathways involved in modulating vascular tone. . $, 8,12-14$ Numerous studies have shown that chronic hypoxia decreases the growth of developing chicken embryos ${ }^{4,7,8,10,15-18}$ and induces a broad spectrum of structural and functional vascular alterations. ${ }^{7,8,14,15,18-20}$ However, the effects of chronic hypoxia on intestinal development, and more particularly on the mesenteric circulation, have been only scarcely investigated. ${ }^{18}$

One key growth factor involved in the pathohysiological effects of hypoxia is vascular endothelial growth factor (VEGF). VEGF is a peptide cytokine that couples hypoxia sensing to angiogenesis in developing and neoplastic tissue. ${ }^{21}$ Evidence from human and experimental studies indicates involvement of VEGF in the pathogenesis of several perinatal complications such as neonatal pulmonary vascular disease, chronic 
lung disease, retinopathy of prematurity, intraventricular hemorrhage and acute renal failure. ${ }^{22}$ In humans, the carrier state for the VEGF-2578 mutant allele, which predisposes to low VEGF production, is associated with the development of NEC in preterm infants. ${ }^{22}$

In a recent study, we analyzed the maturational differences in the reactivity of mesenteric artery (MA) rings isolated from normoxic incubated chicken embryos and hatchlings. ${ }^{23}$ We observed that, as early as 15 of the 21 days of incubation, MAs responded to $\mathrm{K}^{+}$-evoked depolarization and to a wide variety of contractile and relaxant agonists, indicating the presence at this stage of development of electro- and pharmaco-mechanical coupling as well as CGMP- and CAMP-mediated relaxation. In ovo development and transition to ex-ovo life was accompanied by alterations in the response of the MAs but a different developmental trajectory was observed for each reactivity pathway tested. ${ }^{23}$ In the present study we hypothesized that these developmental trajectories would be altered by incubation under hypoxia. We also hypothesized that chronic hypoxia would lead to changes in intestinal morphology and VEGF expression. To test these hypotheses, we analyzed the response to contractile and relaxant agonists of mesenteric arteries from chicken embryos (15 and 19 days) and hatchlings ( $<3$-h-old and 1-day-old) incubated under hypoxic $\left(15 \% \mathrm{O}_{2}\right.$ from day 0 to day 19 of incubation) or normoxic conditions. We also examined the effects of chronic hypoxia on small intestine histology and on the intestinal expression of VEGF. 


\section{METHODS}

\section{INCUBATION OF CHICKEN EMBRYOS}

All experimental procedures were carried out in accordance with the Dutch Law on Animal Experimentation and the European Directive for the Protection of Vertebrate Animals Used for Experimental and Other Scientific Purposes (86/609/EU) and approved by the Committee on Animal Experimentation of the University of Maastricht. Fertilized eggs from White Leghorn chickens ('t Anker, Ochten, The Netherlands) were incubated at $37.8^{\circ} \mathrm{C}, 45 \%$ humidity and rotated once per hour over an angle of $90^{\circ}$ (Incubator model 25HS, Masalles Comercial, Spain). Control embryos were incubated under normoxic conditions $\left(21 \% \mathrm{O}_{2}, 0.03 \% \mathrm{CO}_{2}\right)$. Experimental embryos were incubated in a second 25HS incubator where hypoxic conditions $(15.0 \pm 0.3 \%$ atmospheric $\mathrm{O}_{2}, 0.03 \% \mathrm{CO}_{2}$ ) were maintained by providing a constant flow of $\mathrm{N}_{2}$ and compressed air with a flow meter (AGA Gas BV, The Netherlands). ${ }^{10}$ The $\mathrm{O}_{2}$ and $\mathrm{CO}_{2}$ concentrations in the incubator were monitored with a DrDAQ $\mathrm{O}_{2}$ sensor (Pico Technology, United Kingdom) and an infrared $\mathrm{CO}_{2}$ analyzer (Beckman Instruments, Inc., Fullerton, USA). At day 15 (E15) or 19 (E19) of the 21 days of incubation some embryos were used for experiments and others allowed to hatch. The embryos allowed to hatch were transferred to the normoxic $25 \mathrm{HS}$ incubator at day 19. Hatchlings were used within the first 3 hours (NH3h) or transferred to a brooder unit under $23 \mathrm{~h} /$ day of continuous light and temperature of $35^{\circ} \mathrm{C}$ and provided ad libitum access to water and a standard starter diet. ${ }^{10}$ This last group was studied within the second day posthatch (NH1d).

\section{RECORDING OF MESENTERIC ARTERY REACTIVITY}

On the experimental day, the animals were killed by decapitation, placed on the dorsal side on a Petri-dish coated with silicon and a midline laparotomy and sternotomy were performed. With the aid of a dissecting microscope, the cranial MA was carefully dissected free from surrounding tissue. The cranial MA is the vessel which supplies most of chicken intestine and also the yolk sac during the embryonic period. ${ }^{24}$ Only the intestinal part of the artery was used in our study.

Two stainless steel wires (diameter $40 \mu \mathrm{m}$ ) were inserted into the lumen of the MA, which was mounted as a 1.7-2 mm length ring segment between an isometric force 
transducer and a displacement device in a myograph (Danish Myo Technology A/S model $610 \mathrm{M}$, Aarhus, Denmark). The myograph organ bath $(5 \mathrm{~mL}$ vol) was filled with Krebs-Ringer bicarbonate (KRB, composition in mmol L-1: $\mathrm{NaCl}, 118.5 ; \mathrm{KCl}, 4.75$; $\mathrm{MgSO}_{4} \cdot 7 \mathrm{H}_{2} \mathrm{O}$, 1.2; $\mathrm{KH}_{2} \mathrm{PO}_{4}, 1.2 ; \mathrm{NaHCO}_{3}, 25.0 ; \mathrm{CaCl}_{2}$, 2.5; glucose, 5.5) buffer maintained at $39^{\circ} \mathrm{C}$. After an equilibration period of $30 \mathrm{~min}$, the vessels were distended to a resting tension corresponding to a transmural pressure of $10 \mathrm{mmHg}$ (15-day embryos) or $20 \mathrm{mmHg}$ (19-day embryos and hatchlings). ${ }^{9}$ These pressures correspond to the mean arterial blood pressure reported in chicken at the corresponding age 25 and elicited the highest contractile response to $\mathrm{KCl}$, as determined in pilot experiments. After $30 \mathrm{~min}$. of incubation under basal tone, a control contraction was elicited by raising the $\mathrm{K}^{+}$concentration of the buffer $(62.5 \mathrm{mM})$ in exchange for $\mathrm{Na}^{+}$. During mounting, stabilization, and the experiments, MA rings were maintained in KRB buffer aerated with $95 \% \mathrm{O}_{2} / 5 \% \mathrm{CO}_{2}$.

Contractile agonists were evaluated under basal tone. Concentration-response curves to $\mathrm{KCl}(31.25-125 \mathrm{mM})$, norepinephrine (NE; $10 \mathrm{nM}-0.1 \mathrm{mM})$, the thromboxane $\mathrm{A}_{2}$ mimetic U-46619 (10 nM-3 $\mu \mathrm{M})$, and endothelin (ET)-1 $(0.1 \mathrm{nM}-0.1 \mu \mathrm{M})$ were constructed by increasing the organ chamber concentration of the drug incrementally after a steady-state response had been reached. When two or more agonists were studied in the same arterial preparation, the vessels were repeatedly washed and allowed to equilibrate for at least $30 \mathrm{~min}$. If the tone did not recover to resting level, the vessels were discarded for further experiments.

To study relaxant responses MA rings were contracted with NE $(10 \mu \mathrm{M})$. When contraction reached a plateau, concentration-response curves to acetylcholine (ACh, $10 \mathrm{nM}-0.1 \mathrm{mM}$ ), the NO donor sodium nitroprusside (SNP, $10 \mathrm{nM}-0.1 \mathrm{mM}$ ) and the adenylate cyclase activator forskolin $(10 \mathrm{nM}-10 \mu \mathrm{M})$ were constructed.

\section{MORPHOMETRIC MEASUREMENTS}

After sacrifice, full-thickness segments of terminal ileum were immediately removed, fixed overnight in phosphate-buffered $4 \%$ formaldehyde solution, stored in $70 \%$ ethanol, and embedded in paraffin. Paraffin sections ( $4 \mu \mathrm{m}$ thickness) were cut at 0.1 
$\mathrm{mm}$ intervals, deparaffinized, and stained with hematoxylin and eosin (H\&E). Images were captured using a $5 x$ objective on a standard upright microscope (Leica DM2000) with an attached digital camera (Leica DFC280). Typically, 30 images were captured per tissue section. Using image analysis software (Quantimet 570, Leica), villus height, villus area, number of crypts/villus, and muscularis propria thickness were determined (Fig. 1a) Villi developmental stage was classified using the system of Uni et al. ${ }^{26}$ Briefly, villi were divided into three stages (V1, V2, and V3), differing in length and shape (Fig. 1b), with the larger V1 villi often being pear-shaped and the intermediate V2 villi (approximately $50 \%$ of the size of the V1 villi) being narrower and with a rocketlike shape. The smallest V3 villi were arrow-shaped. Only V1 villi were used for the measurement of length and area. 
A

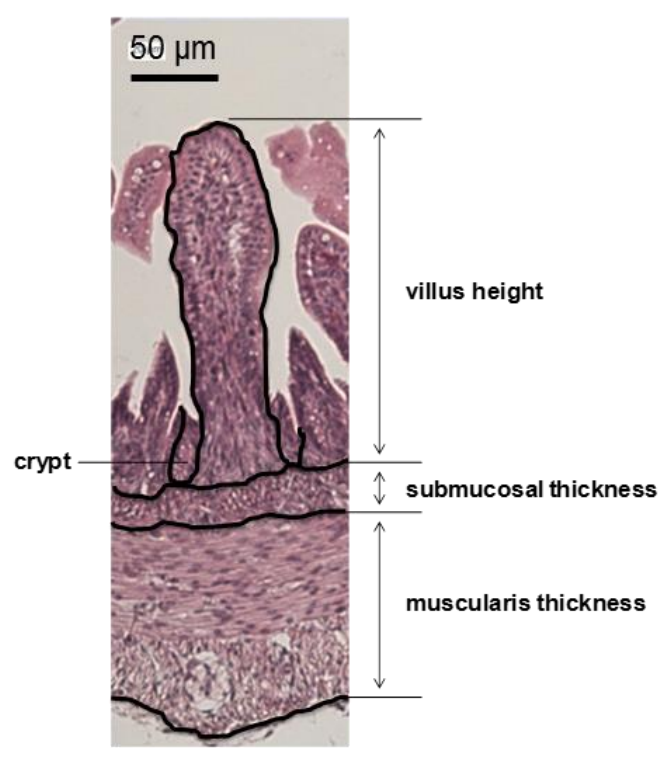

B

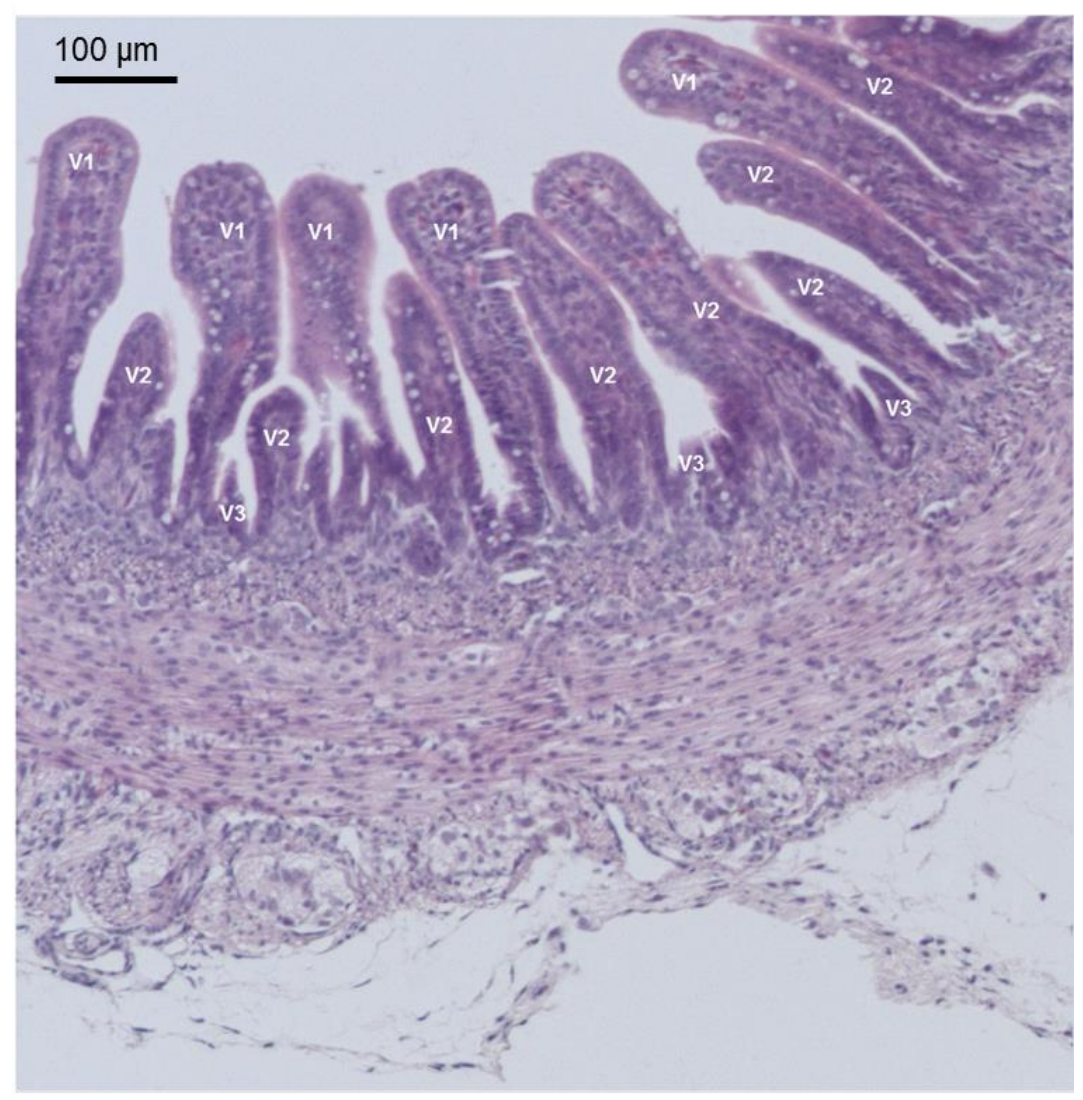

Figure 1. (A) Trace method used to determine villus height, villus area, number of crypts/villus, submucosal thickness and muscularis propria thickness in the chick terminal ileum. (B) Representative light microscopy of intestinal villi from the terminal ileum of a 3-h-old newly hatched chick exposed to normoxia during incubation. V1 to V3 = villi in different stages of development. Staining was with hematoxylin-eosin. 


\section{RNA ISOLATION AND MEASUREMENT OF VASCULAR ENDOTHELIAL GROWTH FACTOR MRNA EXPRESSION}

The chicken VEGF-A gene gives rise to four isoforms generated by alternative splicing of exons (VEGF122, VEGF146, VEGF166, and VEGF190, homologous to human VEGF121, VEGF145, VEGF165, and VEGF189, respectively). ${ }^{27,28}$ We examined the expression of the VEGF-A isoforms by RT-PCR of total RNA isolated from the teminal ileum of normoxic and hypoxic chickens. The ileum section was cut into pieces and tissue RNAs were stabilized in RNAlater ${ }^{\mathrm{TM}}$ (Qiagen, Venlo, The Netherlands) for 1 day at $4^{\circ} \mathrm{C}$, after which the samples were stored at $-80^{\circ} \mathrm{C}$ until use. After thawing, total RNA was isolated using the RNeasy ${ }^{\circledR}$ minikit (Qiagen) according to the manufacturer's protocol, including a DNase treatment step (RNase-free DNase set for use with RNeasy columns; Qiagen). Total RNA was quantified using a Thermo Scientific NanoDrop ${ }^{\mathrm{TM}} 1000$ Spectrophotometer. Part of the RNA was denatured at $65^{\circ} \mathrm{C}$ for 10 min and immediately placed on ice. Then, $500 \mathrm{ng}$ of RNA was translated to cDNA using Ready-To-Go ${ }^{T M}$ You-Prime First-Strand beads (GE Healthcare, Eindhoven, The Netherlands) and $200 \mathrm{pmol} p d(\mathrm{~N})_{6}$ random hexamer primers (GE Healthcare) in a total volume of $25 \mu \mathrm{l}$. RT-PCR was performed at $37^{\circ} \mathrm{C}$ for $1 \mathrm{~h}$, after which the samples were heated to $95^{\circ} \mathrm{C}$ for $3 \mathrm{~min}$ to stop the reaction. Subsequently, samples were cooled to $4^{\circ} \mathrm{C}$ and stored at $4^{\circ} \mathrm{C}$ until use. PCR was performed with VEGF primers (FW 5'caggccatcctgtgtgcctct-3', RV 5'-ttccgctgctcaccgtctccgg-3') designed to yield the four isoforms of avian VEGF. ${ }^{29}$ PureTaq ${ }^{\text {TM Ready-To- Go }}{ }^{\text {TM }}$ PCR beads (GE Healthcare) were used with $10 \mathrm{pmol}$ of each primer and $1 \mu \mathrm{l}$ of $\mathrm{CDNA}$ per reaction with a total volume of $25 \mu \mathrm{l}$. PCR steps were as follows: denaturation $3 \mathrm{~min}$ at $94^{\circ} \mathrm{C}$ (denaturation at $94^{\circ} \mathrm{C} 30 \mathrm{~s}$, annealing at $60^{\circ} \mathrm{C} 1 \mathrm{~min}$, elongation at $72^{\circ} \mathrm{C} 1 \mathrm{~min}$ ). Ribosomal $18 \mathrm{~S}$ was used as an internal standard and analyzed by the same procedure (primer sequences: FW 5'-ccatccaatcggtagtagcg-3', RV 5'-cgataacgaacgagactctgg-3'). PCR products in the exponential phase of the PCR reaction were yielded after 18 cycles. PCR products were visualized at a $2 \%$ agarose gel stained with ethidium bromide and band densities were measured using ImageQuant ${ }^{\mathrm{TM}}$ software (GE Healthcare).

\section{DRUGS AND SOLUTIONS}

Solutions containing different concentrations of $\mathrm{K}^{+}$were prepared by replacing part of the $\mathrm{NaCl}$ of the $\mathrm{KRB}$ buffer by an equimolar amount of $\mathrm{KCl}$. Arterenol bitartrate (NE), 
ACh, and SNP were obtained from Sigma (St. Louis, MO); U-46619 was from Cayman Chemical (Ann Arbor, MI). All drugs were dissolved initially in distilled deionized water (except U46619 in DMSO) to prepare adequate stock solutions and further dilutions were also made in deionized water. The final bath concentration of DMSO did not exceed $0.1 \%$, which we have found elsewhere to have little or no effect on mechanical activity. ${ }^{23}$

\section{DATA ANALYSIS}

Results are shown as mean (SD) of measurements in $n$ animals. For clarity, results are shown in the figures as mean \pm SEM. Contractions are expressed in terms of active wall tension $(\mathrm{mN} / \mathrm{mm})$, calculated as the force divided by twice the length of the segment, while the relaxant responses are expressed as the percentage of reduction of the contraction induced by NE. Sensitivity (expressed as $p E_{50}=-\log E_{50}$ ) and maximal contraction or relaxation $\left(E_{\max }\right)$ to agonists was determined by fitting individual concentration-response data to a non-linear sigmoidal regression curve. Differences in $p \mathrm{EC}_{50}$ and $E_{\max }$ mean values were assessed by the unpaired Student's $\mathrm{t}$-test. If calculation of $p \mathrm{EC50}$ was not appropriate, concentration-response curves were compared by two-way repeated-measures ANOVA (concentration $x$ oxygen level). Data from histology and PCR were analyzed with two-way ANOVA (age $x$ oxygen level), followed by post hoc Bonferroni analysis. Categorical variables were compared by chi-square test. Differences were considered significant at a $P<0.05$. All analyses were performed using GraphPad Prism (version 5.00 for Windows, GraphPad Software, San Diego CA, USA). 


\section{RESULTS}

\section{EFFECTS OF HYPOXIA ON CHICKEN EMBRYO GROWTH}

Exposure of chicken embryos, from the first day of incubation, to $15 \%$ instead of $21 \%$ $\mathrm{O}_{2}$ induced a significant decrease of body mass at E15 and E19 (Table 1). When the hypoxic embryos were incubated under normoxia (from day 19 onward), no significant differences in body mass were observed at $\mathrm{NH} 3 \mathrm{~h}$ or $\mathrm{NH} 1 \mathrm{~d}$ (Table 1).

Table 1. Effects of hypoxia during incubation in the body mass of 15-day (E15) and 19-day (E19) chicken embryos and 3-h-old (NH3 h) and 1-day-old (NH1d) newly hatched chicks

\begin{tabular}{cccccccccc}
\hline & \multicolumn{2}{c}{ E15 } & \multicolumn{2}{c}{ E19 } & \multicolumn{2}{c}{ NH3h } & \multicolumn{2}{c}{ NH1d } \\
\cline { 2 - 9 } & $\begin{array}{c}\text { control } \\
(\mathrm{n}=40)\end{array}$ & $\begin{array}{c}\text { hypoxia } \\
(\mathrm{n}=30)\end{array}$ & $\begin{array}{c}\text { control } \\
(\mathrm{n}=47)\end{array}$ & $\begin{array}{c}\text { hypoxia } \\
(\mathrm{n}=31)\end{array}$ & $\begin{array}{c}\text { control } \\
(\mathrm{n}=50)\end{array}$ & $\begin{array}{c}\text { hypoxia } \\
(\mathrm{n}=35)\end{array}$ & $\begin{array}{c}\text { control } \\
(\mathrm{n}=16)\end{array}$ & $\begin{array}{c}\text { hypoxia } \\
(\mathrm{n}=15)\end{array}$ \\
\hline Body & 12.99 & $12.08^{*}$ & 27.97 & $24.82^{*}$ & 41.82 & 41.45 & 40.25 & 38.74 \\
Mass & SD & SD & SD & SD & SD & SD & SD & SD \\
(g) & 0.93 & 1.17 & 2.85 & 1.87 & 3.16 & 2.81 & 2.92 & 2.17 \\
\hline
\end{tabular}

${ }^{*} P<0.001$ vs control (normoxia).

\section{MESENTERIC ARTERY REACTIVITY}

The developmental changes in MA reactivity have been described in a recent publication of our laboratory. ${ }^{23}$ Therefore, in this work we focused on the effects of hypoxic incubation in MA reactivity. The contractions evoked by $\mathrm{KCl}$ (Fig. 2a-d), and U-46619 (Fig. 2e-h) were not affected by hypoxic incubation. In contrast, hypoxic incubation induced a significant increase in the effectivity $\left(E_{\max }\right)$ of NE at $\mathrm{E} 19$ ( $E_{\max }$ normoxia: $1.23 \mathrm{mN} / \mathrm{mm}$, SD 0.61, $n=6$; $E_{\max }$ hypoxia: $2.08 \mathrm{SD} 0.49 \mathrm{mN} / \mathrm{mm}, n=8 ; P$ $<0.01$; Fig. 3b). No effects of hypoxia on NE responsiveness were observed at the other ages studied (Fig. 3). Hypoxia also induced a significant increase in the responsiveness to $\mathrm{ET}-1$ at $\mathrm{E} 15$ (Fig. $4 \mathrm{a}$ ) and $\mathrm{NH} 1 \mathrm{~d}$ ( $E_{\max }$ normoxia: $0.40 \mathrm{mN} / \mathrm{mm}$, SD 0.16, $n=7$; $E_{\max }$ hypoxia: $0.73 \mathrm{mN} / \mathrm{mm}, \mathrm{SD} 0.23, n=6 ; P<0.01$; Fig. $4 \mathrm{~d}$ ). Moreover, 
high concentrations $(\geq 0.1 \mu \mathrm{M})$ of ET-1 induced relaxation in the MA from NH1d chicks (Fig. 4e) but this relaxant effect of ET-1 is not observed after hypoxic incubation (Fig. $4 f)$.

In the above-mentioned study, we demonstrated that ACh evoked endotheliumdependent relaxation in MA from embryonic and $\mathrm{NH}$ chickens. ${ }^{23}$ Herein, we observed that the sensitivity to ACh was significantly diminished in the hypoxic E15 animals ( $p \mathrm{EC}_{50}$ normoxia: $6.44, \mathrm{SD} 0.24, n=11, p \mathrm{EC}_{50}$ hypoxia: $5.73, \mathrm{SD} 0.24, n=6 ; P<$ 0.001 , Fig. 5a). Hypoxia did not affect the sensitivity to $A C h$ at the other ages examined (Fig. 5b-d) but induced a slight decrease in the $E_{\max }$ at NH1d (Fig. $5 d$ ). As shown in Fig. $5 f$ the sensitivity to the NO-donor SNP was significantly greater in the hypoxic E19 ( $p \mathrm{EC}_{50} 7.31$, SD $0.34, n=7$ ) embryos when compared with their respective agematched controls ( $p \mathrm{EC}_{50} 6.05$, SD 0.32, $n=10 ; P<0.001$ ). Hypoxia did not affect the responsiveness to SNP at E15, NH3h or NH1d (Fig. 5e, 5g-h). 
A

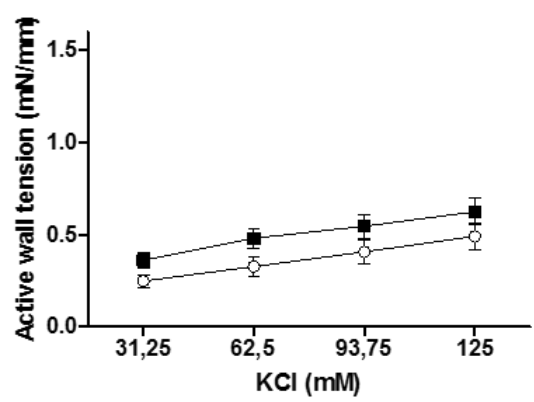

C

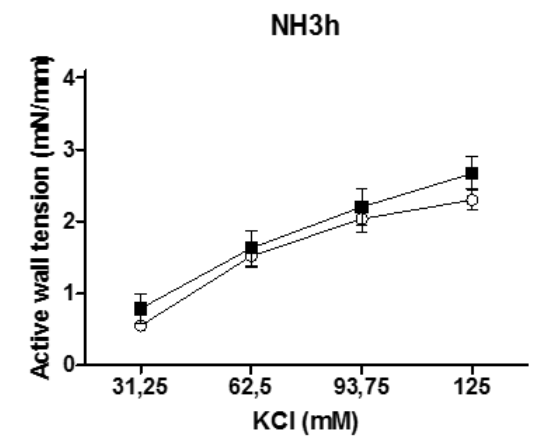

E

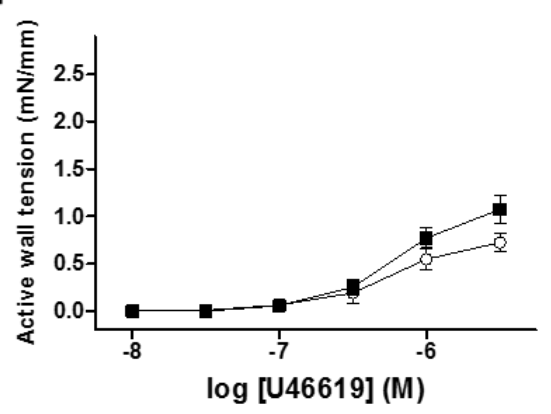

G

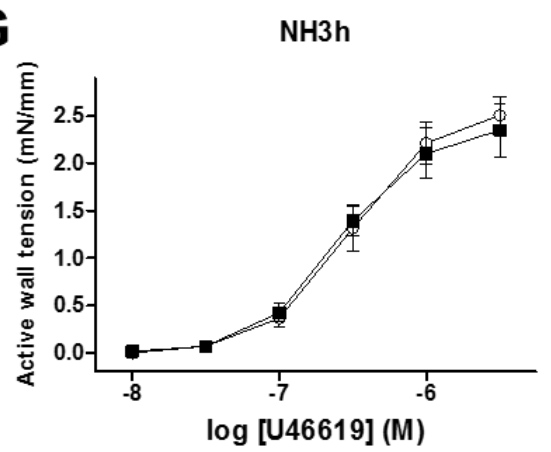

B

-o- normoxia

- hypoxia

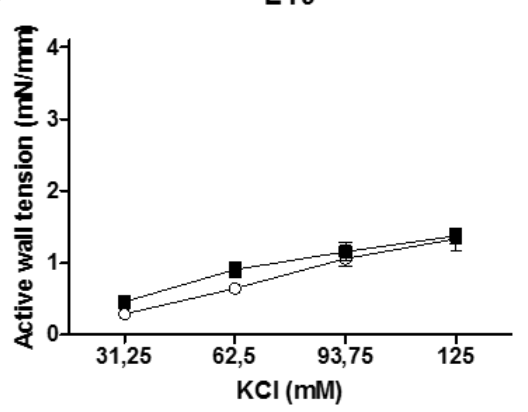

D

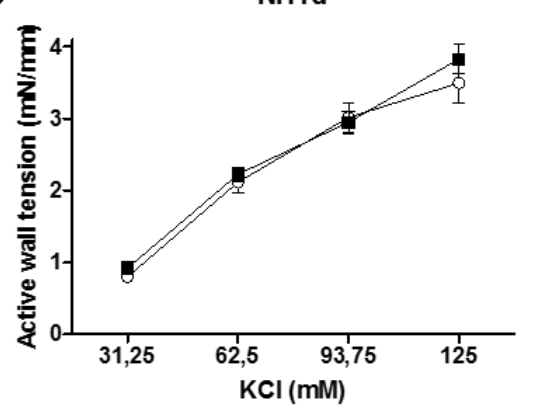

$\mathbf{F}$

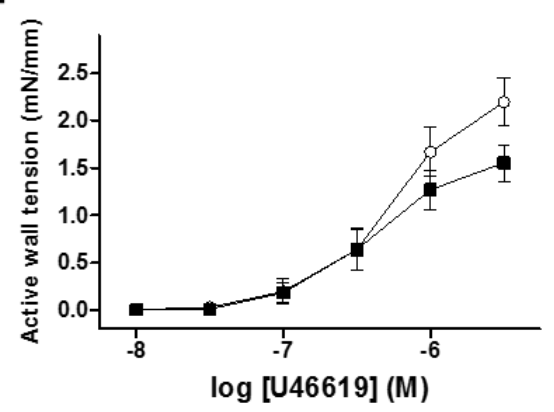

H

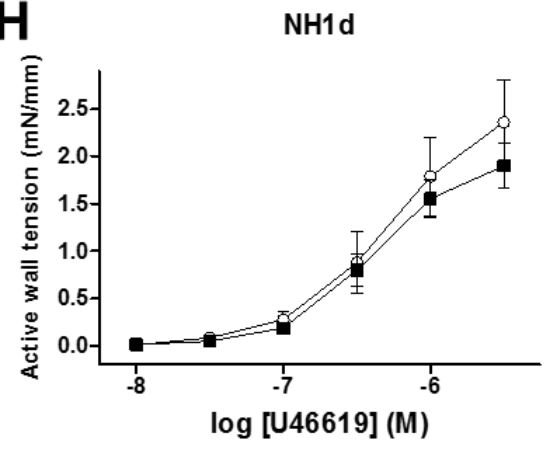

Figure 2. Concentration-dependent contractile effects of $\mathrm{KCl}$ and the thromboxane $\mathrm{A} 2$ mimetic U46619 in mesenteric artery rings from 15-day (E15) and 19-day (E19) chicken embryos and 3-h-old (NH3 h) and 1-day-old (NH1d) newly hatched chicks exposed to hypoxia (-) or normoxia (०) during incubation. Each point represents the mean \pm SEM of 5-8 embryos/chicks. 
A

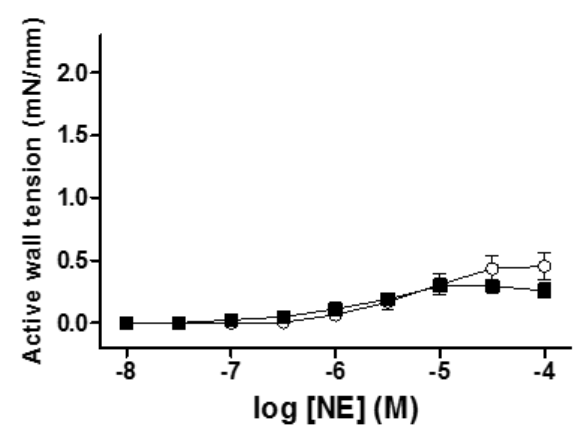

C

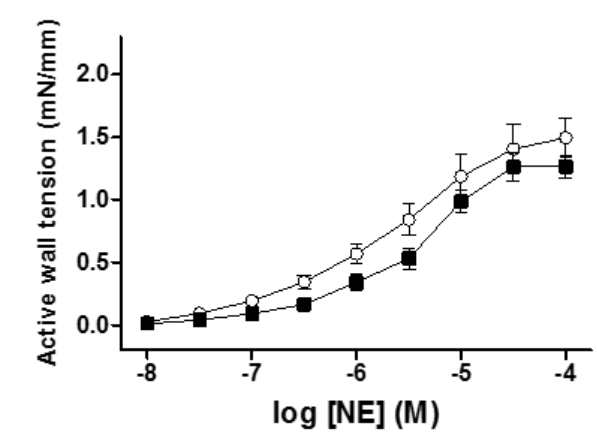

- normoxia

- hypoxia

B

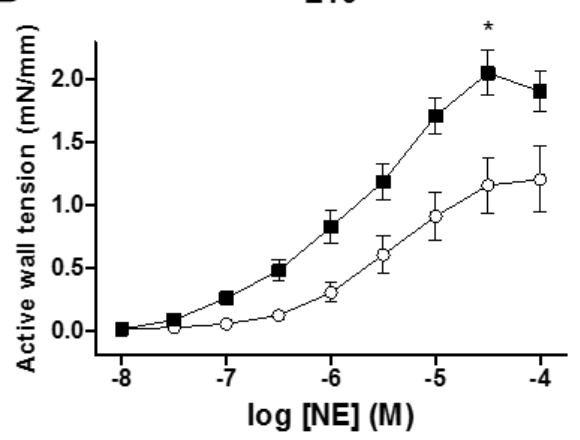

D

NH1d

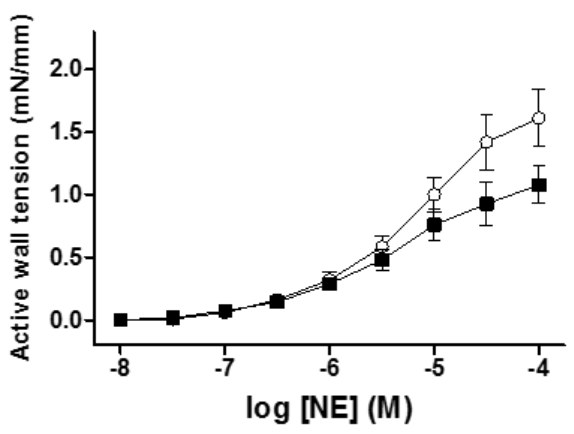

Figure 3. Concentration-dependent contractile effects of norepinephrine (NE) in mesenteric artery rings from 15-day (E15) and 19-day (E19) chicken embryos and 3-h-old (NH3 h) and 1day-old (NH1d) newly hatched chicks exposed to hypoxia (-) or normoxia (0) during incubation. Each point represents the mean \pm SEM of 5-8 embryos/chicks. ${ }^{*} P<0.05$ for difference (assessed by $t$-test) in $E_{\max }$ compared with normoxia. 
A

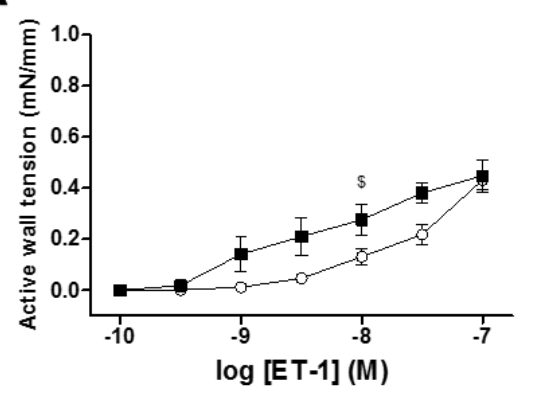

C

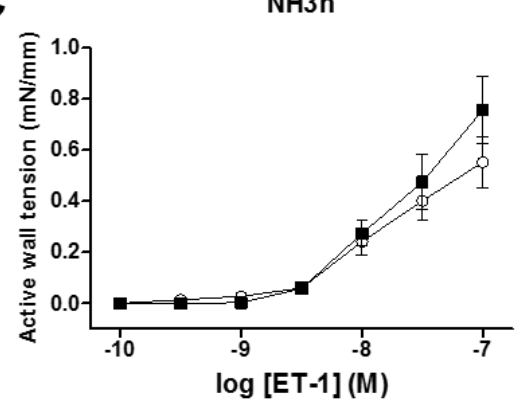

B

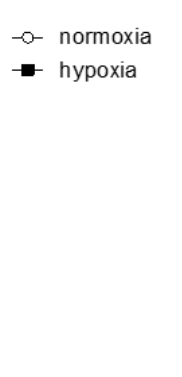

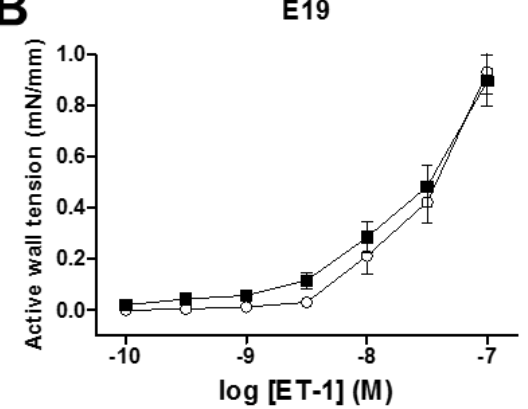

D

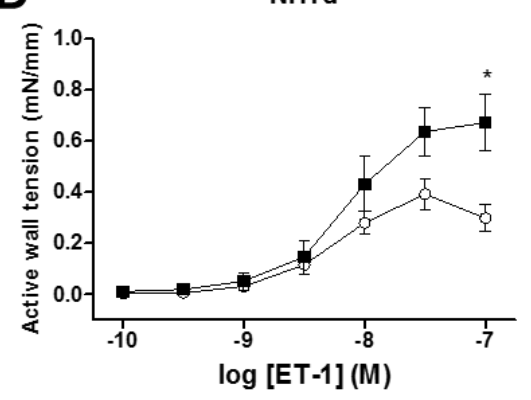

$\mathbf{E}$

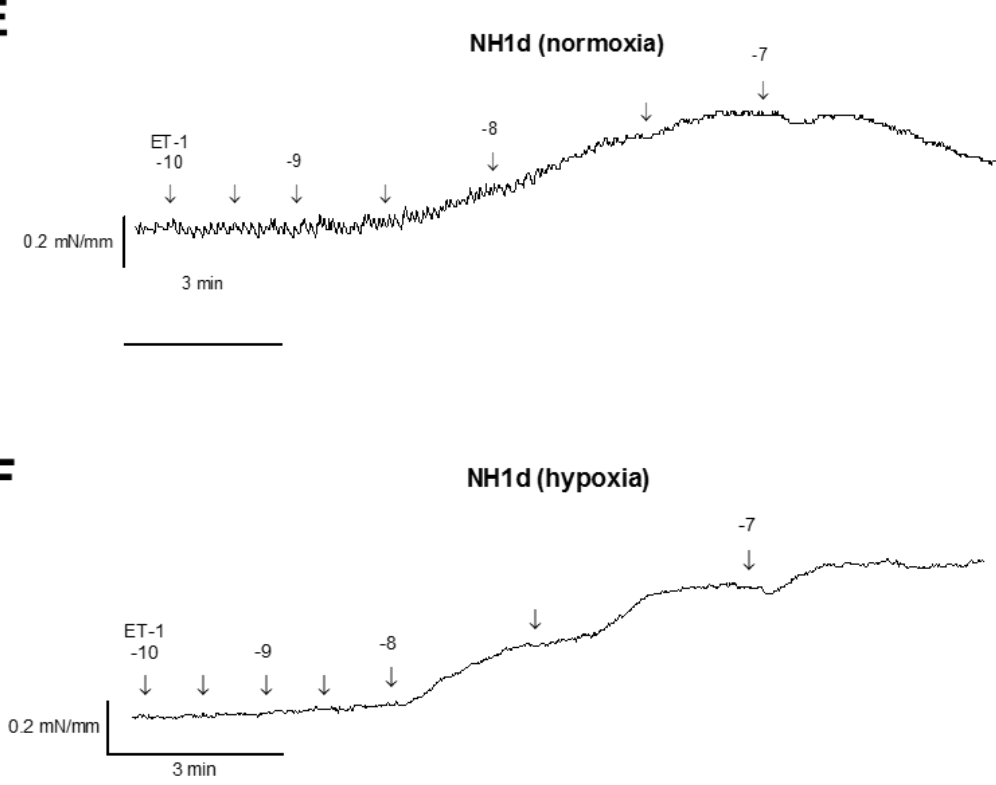

Figure 4. Concentration-dependent contractile effects of endothelin-1 (ET-1) in mesenteric artery rings from 15-day (E15) and 19-day (E19) chicken embryos and 3-h-old (NH3 h) and 1day-old (NH1d) newly hatched chicks exposed to hypoxia (-) or normoxia (0) during incubation. Each point represents the mean \pm SEM of 6-12 embryos/chicks. ${ }^{*} P<0.05$ for difference (assessed by $t$-test) in $E_{\max }$ compared with normoxia. $\$ P<0.05$ for difference (assessed by two-way ANOVA) in the overall contractile response compared with normoxia. In panels $(E)$ and $(F)$ (representative tracings), numbers above the arrows indicate log M [ET1] and arrows without numbers indicate half-log increments in concentration. 
A

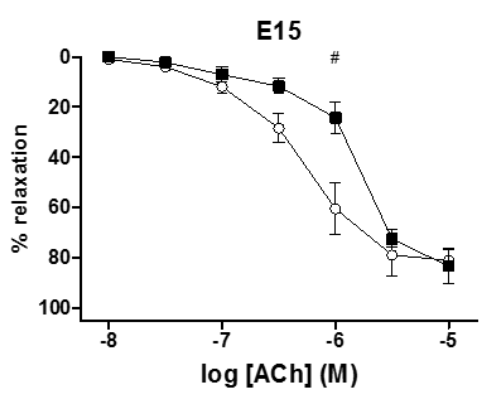

C

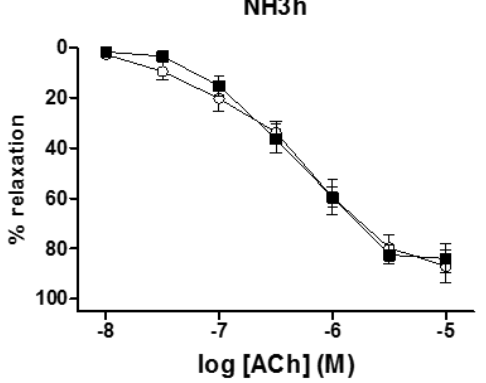

$\mathbf{E}$

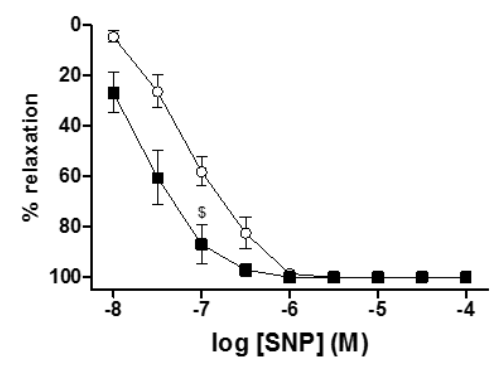

G

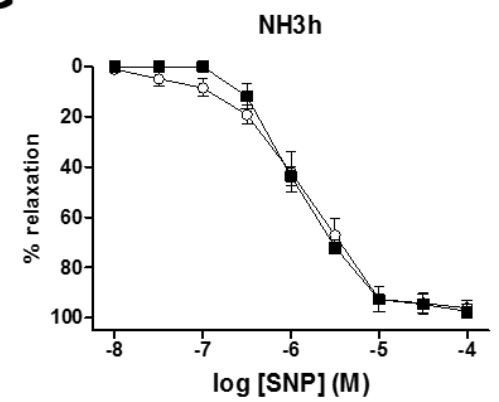

B

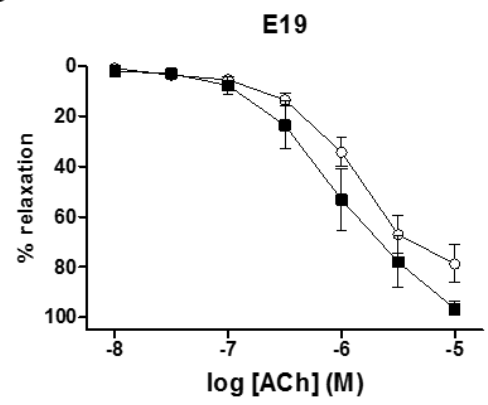

D

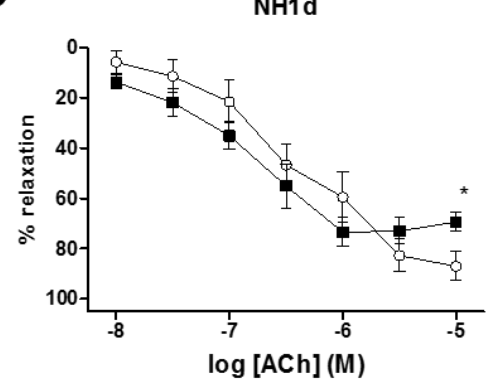

$\mathbf{F}$

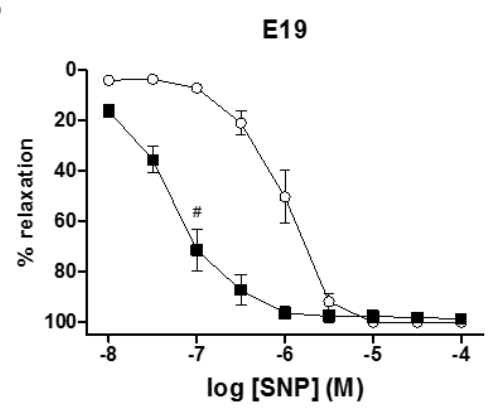

H

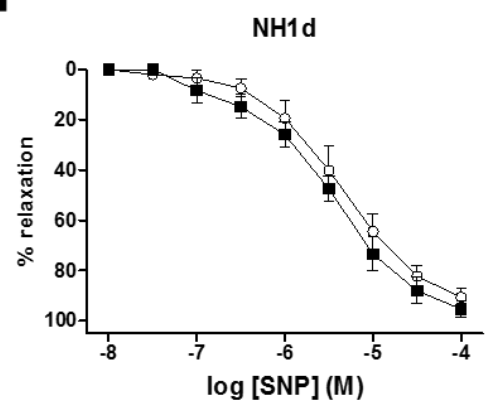

Figure 5. Concentration-dependent relaxant effects of acetylcholine (ACh) and the nitric oxide donor sodium nitroprusside (SNP) in mesenteric artery rings from 15-day (E15) and 19-day (E19) chicken embryos and 3-h-old (NH3 h) and 1-day-old (NH1d) newly hatched chicks exposed to hypoxia (घ) or normoxia (०) during incubation. Each point represents the mean \pm SEM of 4-10 embryos/chicks. ${ }^{*} P<0.05$ for difference (assessed by $t$-test) in $E_{\max }$ compared with normoxia. \#P $<0.05$ for difference (assessed by t-test) in pEC50 compared with normoxia. $\$ P<0.05$ for difference (assessed by two-way ANOVA) in the overall relaxant response compared with normoxia. 


\section{MORPHOLOGICAL EXAMINATION}

Histological analysis indicated that the chicken embryo terminal ileum was growing rapidly. Villi at E15 were rudimentary and crypts were not present (Fig. 6a). In the E19 intestine, only a few crypts were observed. These crypts were small and contained few cells (Fig. 6b). In the E15 and the E19 intestines only V1 and V2 villi were present and the distribution of these two villus stages did not change significantly between the two ages (Fig. 7f). The terminal ileum of the hatchlings ( $\mathrm{NH} 3 \mathrm{~h}$ and $\mathrm{NH} 1 \mathrm{~d}$ ) showed a marked increase in size and complexity with longer fingerlike villi and, generally, more than one crypt per villlus in the intervillous epithelium (Fig. 6c-d, Fig. 7). Villi at the three stages of development (V1, V2 and V3) were present in a similar proportion at the two hatchling ages tested (Fig. 7f). Numerous goblet cells were observed on the villi surface of the hatchlings (Fig. 6c-d).

Ileal morphology of the hypoxic animals was, in general, comparable to that of controls (Fig. 7). The only exceptions to this were a small, but significant, decrease in villus height and muscularis propria thickness that was observed in the NH3h hypoxic animals (Fig. 7a-e). The distribution of the three villus stages did not change between hypoxic and normoxic animals (Fig. 7f). 
E15

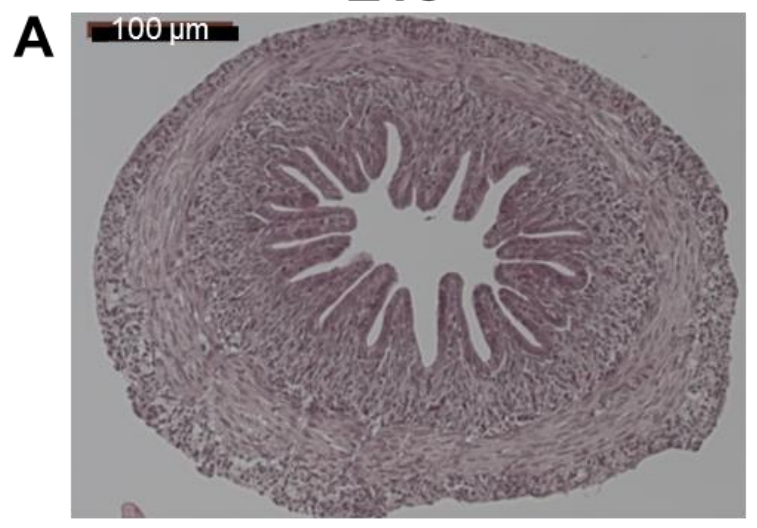

NH3h

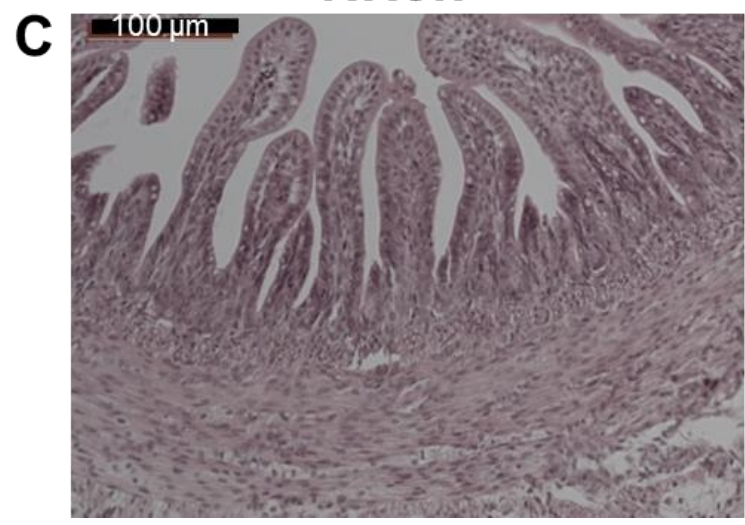

E19

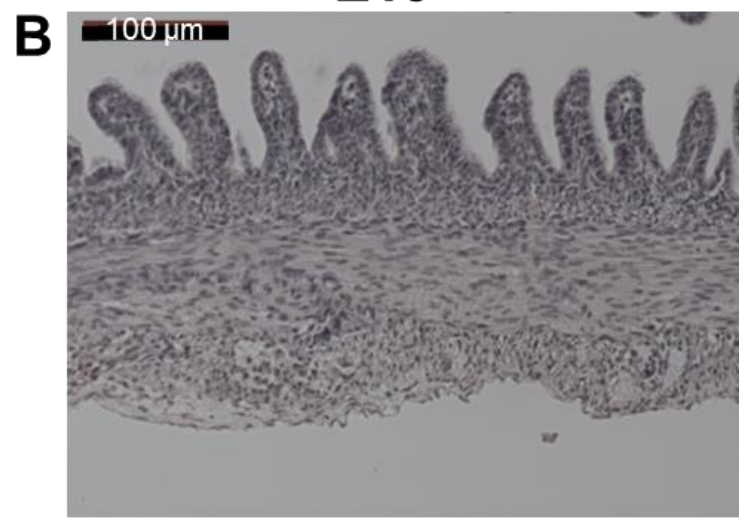

NH1d

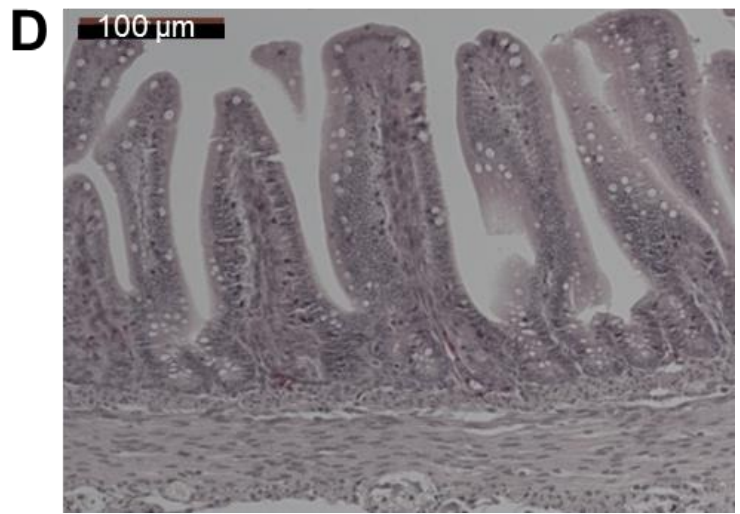

Figure 6. Representative light microscopy of the terminal ileum of 15-day (A) and 19-day (B) chicken embryos and 3-h-old (C) and 1-day-old (D) newly hatched chicks exposed to normoxia during incubation. Sections of were stained with hematoxylin and eosin. Bar $=100 \mu \mathrm{m}$. 
A

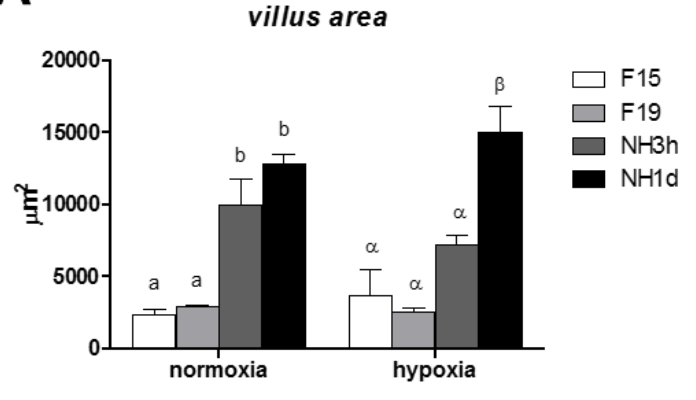

C

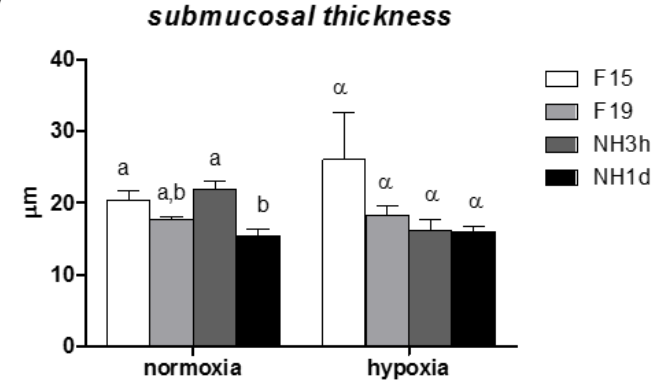

E

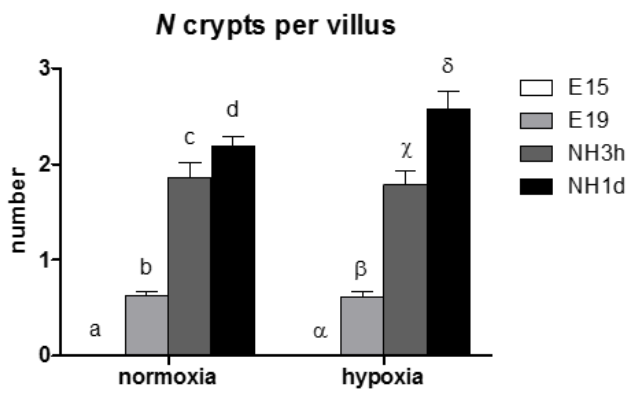

B

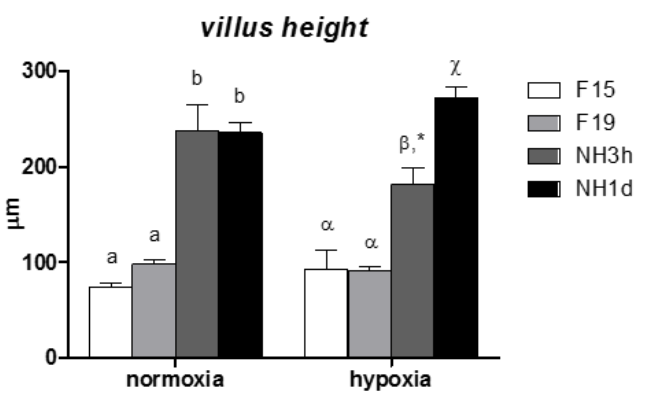

D

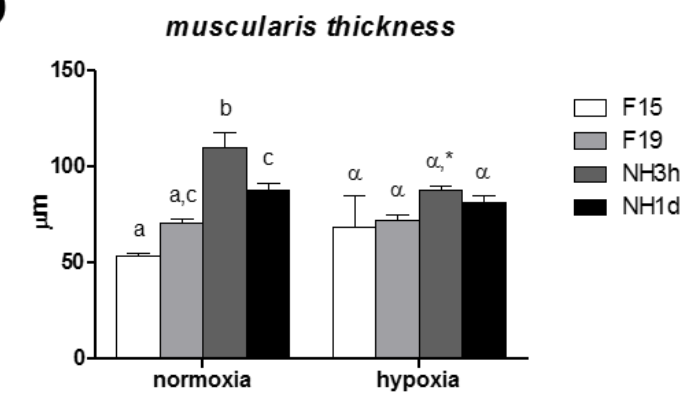

$\mathbf{F}$

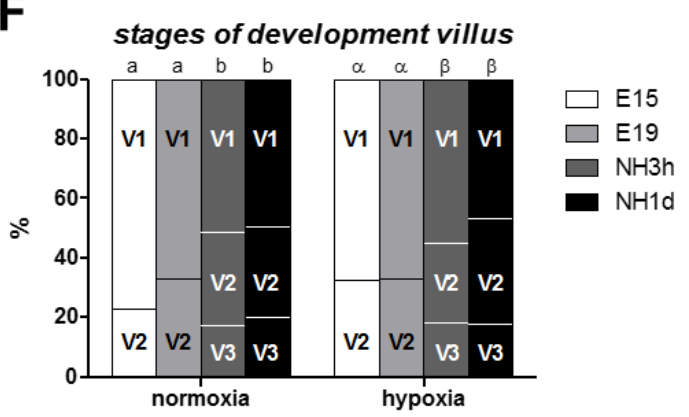

Figure 7. Morphological measurements of the terminal ileum of 15-day (E15) and 19-day (E19) chicken embryos and 3-h-old (NH3 h) and 1-day-old (NH1d) newly hatched chicks exposed to hypoxia or normoxia during incubation. Each bar represents the mean \pm SEM of 4-8 embryos/chicks. Values without a common letter ( $a, b, c, d$ for normoxia and $\alpha, \beta, \gamma, \delta$ for hypoxia) are significantly different $(p<0.05)$. ${ }^{*}<<0.05$ compared with normoxia within the same age group. 


\section{ILEAL VASCULAR ENDOTHELIAL GROWTH FACTOR MRNA EXPRESSION}

VEGF mRNA expression was investigated in the ileum of normoxic E15 (n=8), E19 $(n=9)$ and NH3d $(n=10)$ chicks and hypoxic $\mathrm{E} 15(n=7), \mathrm{E} 19(n=8)$ and NH3d $(n=12)$ chicks. When the mRNA expression of the VEGF-A isoforms was examined by RTPCR, we detected two bands corresponding to VEGF122 and VEGF166 isoforms (Fig. 8a). No signal was detected for the VEGF146 and VEGF190 isoforms. As shown in fig. 8b, there was a trend toward higher levels of VEGF122 expression in the ileum of the $\mathrm{NH}$ compared with embryonic chicks, but this did not reach statistical significance. The expression of VEGF166 was significantly higher in the NH chicks (Fig 8c). This developmental increase in VEGF was impaired by hypoxic incubation. Thus VEGF expression was significantly lower in the $\mathrm{NH}$ hypoxic chicks compared with normoxic controls (Fig. 8). 
A

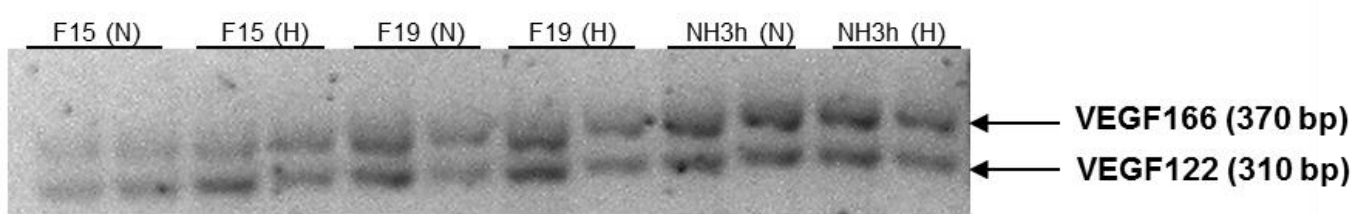

B

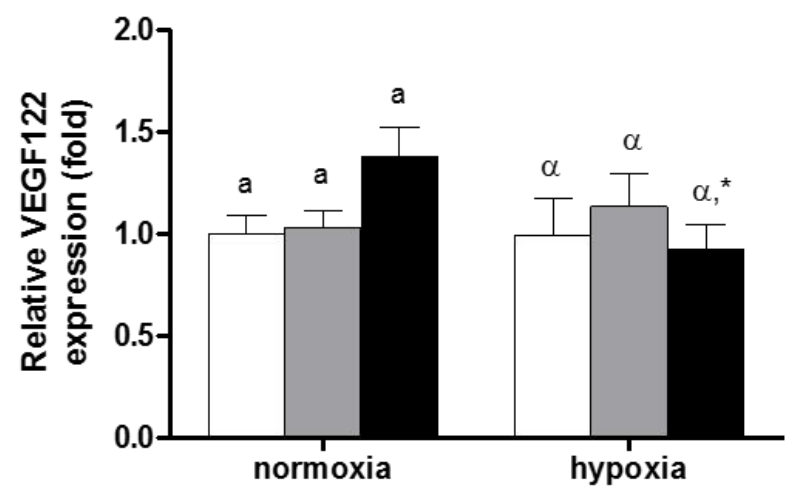

C

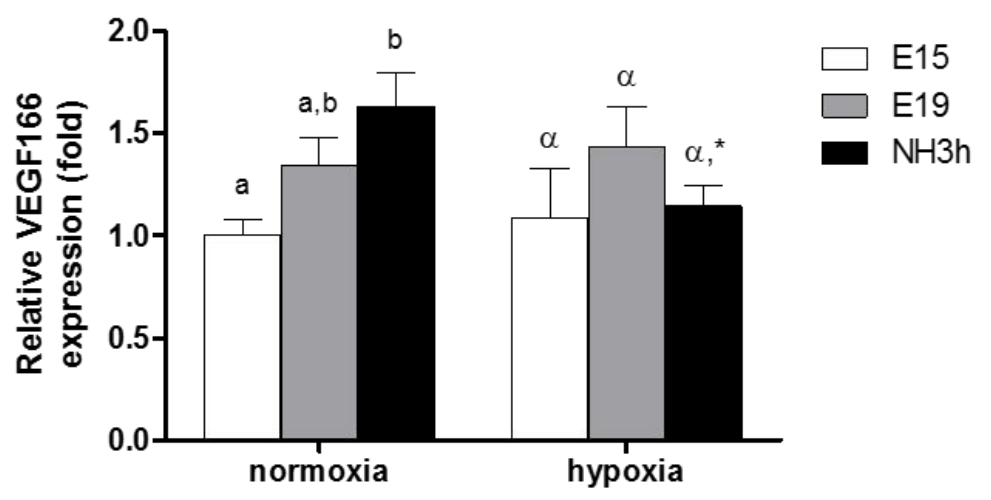

Figure 8. (A). VEGF isoforms mRNA expression in the chicken terminal ileum. VEGF isoforms, 122 (240bp) and 166 (370bp) were detected, but not isoform 146 (310bp) or 190 (442bp). (B, C) VEGF expression patterns of the terminal ileum of 15-day (E15) and 19-day (E19) chicken embryos and 3-h-old (NH3 h) newly hatched chicks exposed to hypoxia or normoxia during incubation. Each bar represents the mean \pm SEM of 7-12 embryos/chicks. Values without a common letter ( $a, b, c$, for normoxia and $\alpha, \beta, \gamma$ for hypoxia) are significantly different $(p<0.05)$. ${ }^{\star} P<0.05$ compared with normoxia within the same age group. 


\section{DISCUSSION}

Structural and functional changes occur in the prenatal intestine with development in preparation for enteral feeding following birth. Afterwards, the postnatal development of the gastrointestinal system is a very dynamic process in which an intense growth and remodeling of the tissues take place. ${ }^{30}$ The present study focused on how intestinal development of chicken embryos and hatchlings is affected by chronic moderate hypoxia $\left(15 \% \mathrm{O}_{2}\right)$ during incubation. Hypoxia altered the responsiveness of chicken embryo mesenteric arteries to the adrenergic agonist $\mathrm{NE}$, the endotheliumdependent relaxant agonist acetylcholine, the NO donor SNP and the constrictor polypeptide ET-1. However, the majority of these alterations, with the exception of the hyperresponsiveness to ET-1, were not present in the hypoxic hatchlings. When intestinal histology was analyzed, subtle hypoxia-induced changes were noted in the villi and the muscularis from the hatchlings. Hypoxic incubation also diminished the expression of VEGF mRNA in the terminal ileum of the hatchlings.

In our study, we deliberately chose for a model in which hypoxia was not applied from day 19 onward. Unlike the rapid transition from an intrauterine to an extrauterine environment displayed in most mammals, bird hatching from eggs is an event that may take place over several days. ${ }^{31,32} \mathrm{O}_{2}$ demand increases exponentially during development, and it exceeds the capacity of the choroallantoic membrane gaseous diffusion by the end of the avian incubation period. ${ }^{32}$ At this point, around day 19 of incubation in the chicken, the beak of the embryo penetrates the air cell, air enters the lung, and breathing is initiated. This process is termed internal pipping and is followed by external pipping when an opening of the shell is achieved and ambient air is breathed for the first time..$^{31,32}$ In our experiments, both normoxic and hypoxic embryos were incubated under $21 \% \mathrm{O}_{2}$ between days 19 and 21 to avoid the interference of hypoxia with the processes of internal and external pipping (i.e., to avoid alveolar hypoxia) and because hypoxia during the last 2 days of incubation produces a dramatic decrease in hatchability. ${ }^{8,10,14}$ Nevertheless, it is also known that these two days of normoxic incubation are sufficient to revert some of the effects of hypoxia on growth and vascular responsiveness. ${ }^{8,10} \mathrm{~A}$ possible explanation for this reversion of the effects of hypoxic incubation might be a delayed hatching in the hypoxic group which allowed 
a longer exposure to normoxia between day 19 and hatching. We do not know if this was the case in our study because our experimental design did not include assessment of hatching time. Molenaar et al analyzed this issue in chicken embryos incubated between day 7 and day 19 under 21 or $17 \% \mathrm{O}_{2}$ and found no significant differences in hatching time. ${ }^{33}$ In the present study, we observed that the hypoxia-induced reduction in body mass and the alterations in the responsiveness to NE, ACh and SNP were only present in the prenatal period but not in the $\mathrm{NH}$ chicks. In contrast, the responsiveness to ET-1 was altered in the embryonic and the $\mathrm{NH}$ chicks and the changes in intestinal morphology and VEGF expression were only observed in the NH chicks. Altogether, our data suggest that chronic hypoxia during incubation altered the developmental trajectory of chicken intestine and that this altered development leaded to changes in intestinal homeostasis that are present in the post-hatch period.

\section{EFFECTS OF HYPOXIA ON CONTRACTILE RESPONSES OF MESENTERIC}

\section{ARTERIES}

The redistribution of fetal cardiac output during hypoxia may be partially dependent on increased circulating levels of several vasoactive factors, including NE and ET-1, but may also involve changes in the local vascular responses to these circulating factors. ${ }^{34}$ In the present work, we observed that the contractile response evoked by $\mathrm{K}^{+-i n d u c e d}$ depolarization was similar in the normoxic and the hypoxic MAs. $\mathrm{K}^{+}$-induced contraction is often used to examine the smooth muscle contraction function and to standardize the receptor-mediated contraction. ${ }^{23}$ Our data suggest, therefore, that the intrinsic contractile properties of the MAs are not affected by hypoxic incubation. In contrast, chronic hypoxia increased the responsiveness to NE in the E19 and to ET-1 in the E15, E19 and NH1d chicks.

Exposure to mild chronic hypoxia during incubation resulted in increased NE levels in E19 chicks $^{35}$ suggesting that catecholamines are involved in the prenatal adaptation to chronic hypoxia in this species. However, the alterations in vascular adrenergic responsiveness that accompany the increase in catecholamines are markedly tissuedependent. Thus, hypoxia increased adrenergic contraction in the MA (present work) and the DA of the E19, but did not affect the adrenergic responssiveness of the femoral 
and the pulmonary arteries. ${ }^{8,14}$ Tintu et al. ${ }^{18}$ analyzed the reactivity of second-order mesenteric resistance arteries from chicken embryos incubated under normoxia or hypoxia. They observed that the hypoxic E19 MAs showed an enhanced vasoconstriction in response to the sympathetic nervous stimulator tyramine and an enhanced vasodilation in response to the $\alpha$-adrenoceptor blocker phentolamine. These data suggest an increased activity of perivascular sympathetic nerves in chronically hypoxic embryos at this stage of development. Our present results suggest that in larger arteries an increased sensitivity to cathecholamines is also part of the spectrum of alterations induced by chronic prenatal hypoxia in the mesenteric circulation.

ET-1 is a vasoactive and mitogenic polypeptide produced mainly by the vascular endothelium. Its binding to $\mathrm{ET}_{\mathrm{A}}$ and $\mathrm{ET}_{\mathrm{B}}$ receptors on vascular smooth muscle induces contraction, whereas its binding to endothelial $\mathrm{ET}_{\mathrm{B}}$ receptors causes vasodilatation. ${ }^{36}$ ET-1 is constitutively produced, but its production can also be stimulated by a wide range of stimuli, including hypoxia. ET-1 levels in amniotic fluid in the human near-term fetus have been found to inversely correlate with $\mathrm{PO}_{2}$ in serum as measured by cordocentesis. ${ }^{37}$ In hypoxic pulmonary hypertension in newborn mice, rats, and piglets, alternations in ET-1 level and $\mathrm{ET}_{\mathrm{A}}$ receptor binding indicate that they may be responsible for the increased pulmonary vasoconstriction and vascular remodeling. ${ }^{38}$ In some mammalian species, ET-1 is considered as the primary vasoconstrictor stimulus in the perinatal intestinal circulation. ${ }^{36}$ In the chicken embryo, ET-1 evoked contraction in the mesenteric ${ }^{23}$, the pulmonary ${ }^{39}$, the femoral, and the chrorioallatoic arteries $^{9}$, as well as in the ductus arteriosus. ${ }^{40}$ In the MA from normoxic chicks, ET-1induced contraction reached a peak at E19 and decreased in the hatchlings. Moreover, high concentrations of ET-1 induced relaxation in the MA from $\mathrm{NH} 1 \mathrm{~d}$ chicks ${ }^{23}$ but this relaxant effect of ET-1 is not observed after hypoxic incubation (Fig. 4f). Although, we have not characterized the nature of the response to ET-1, our previous results suggested the presence of ETA-mediated contraction in the embryonic arteries and the

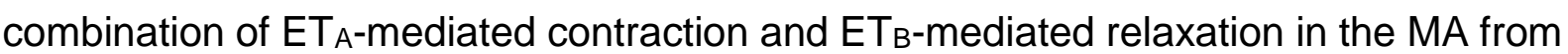

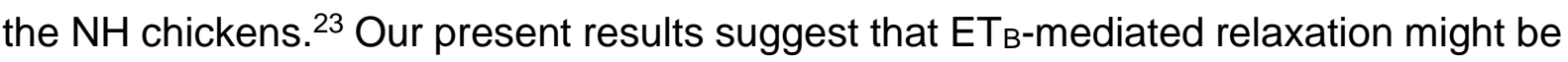
impaired by chronic prenatal hypoxia. Accordingly, prolonged exposure to hypoxia increased the contractile effect of ET-1 and abolished relaxant effect mediated by the $\mathrm{ET}_{\mathrm{B}}$ receptor in murine and porcine pulmonary arteries. ${ }^{41,42}$ Our present findings in the 
MA from hypoxic chicks warrant further investigation into the constitutive production of $\mathrm{ET}-1$ and the hypoxia-induced changes in $\mathrm{ET}_{\mathrm{A}}$ and $\mathrm{ET}_{\mathrm{B}}$ receptor expression.

\section{EFFECTS OF HYPOXIA ON RELAXANT RESPONSES OF MESENTERIC ARTERIES}

In the chicken embryo ACh induced an endothelium-dependent and, at least partially, NO-mediated relaxation of the mesenteric ${ }^{23}$, the pulmonary ${ }^{39}$, the femoral ${ }^{39}$, the carotid arteries $^{43}$ and the ductus arteriosus. ${ }^{11}$ As reported elsewhere, chronic moderate hypoxia in the chicken embryo led in the femoral artery ${ }^{7,8}$ and the ductus arteriosus ${ }^{44}$ to impairment of $\mathrm{ACh}$-induced relaxation. In contrast, $\mathrm{ACh}$-induced relaxation of chicken embryo pulmonary arteries was not affected by chronic hypoxic incubation. ${ }^{8}$ Herein, we observed diminished sensitivity to ACh in the E15 hypoxic animals. Interestingly, the sensitivity to the endothelium-independent soluble guanylate cyclase (sGC) stimulator SNP was significantly increased in the MAs from E19 hypoxic embryos. This effect can be attributed to an enhanced sensitivity or expression, of sGC and could have partially masked the impairment of ACh-induced relaxation in the hypoxic MAs. The work from numerous laboratories shows that the activation of endothelium-independent pathways may compensate for the loss of endotheliumdependent relaxation. ${ }^{45-47}$ In opposition with our present results, chronic hypoxia did not affect SNP-mediated relaxation in chicken embryo femoral and pulmonary arteries and impaired it in the ductus arteriosus. ${ }^{7,8,19,20}$ Altogether, this suggests that the effects of hypoxia in endothelium-dependent and -independent relaxation are strongly vascular bed-dependent.

\section{EFFECTS OF HYPOXIA ON INTESTINAL MORPHOLOGY}

The development of the gastrointestinal tract in pre- and posthatch chicks has been described by several authors. ${ }^{26,48-51}$ Between days 17 and 19 of incubation, a progressive but slow increase in villus development is observed. ${ }^{26}$ Additional rapid morphological changes occur during later embryonic development and, specially, after hatch. ${ }^{26,48-51}$ The accelerated rate of development posthatch is reflected in the severalfold elevation in numbers of enterocytes during first few days posthatch, resulting from 
the dramatic increase in villus length and surface. ${ }^{50}$ Our present results confirm this pattern of pre- and posthatch development.

Tibboel et al. reported that from day 14 of incubation onward an acute $(3 \mathrm{~h})$ exposure to $12-13 \% \mathrm{O}_{2}$ produced serious necrotic changes in the intestine of the chicken embryo. ${ }^{52}$ In contrast, when we investigated the effects of chronic exposure to $15 \% \mathrm{O}_{2}$ on the morphological development of chick terminal ileum, we only observed subtle alterations. In fact, prehatch intestinal development was similar in normoxic and hypoxic embryos. After hatching, the hypoxic chicks showed decreased villus length and muscularis propria thickness (only observed at NH3h). We can only speculate on the functional significance of these subtle hypoxia-induced alterations. An important consideration is that, in parallel with the morphological changes, the ability of the intestinal tissue to digest and absorb nutrients increases steadily during the first days posthatch.50,51 Interestingly, Molenaar et al. demonstrated that the efficiencies of nutrient utilization for growth improved in the posthatch period in chicken embryos incubated between day 7 and 19 at $17 \% \mathrm{O}_{2}$ when compared with normoxic-incubated chicks. ${ }^{33}$ This suggests that embryos incubated under suboptimal environmental conditions may develop adaptive mechanisms that still continue in the posthatch period. ${ }^{33}$

\section{EFFECTS OF HYPOXIA ON INTESTINAL VASCULAR ENDOTHELIAL GROWTH FACTOR EXPRESSION}

We found that intestinal VEGF expression increased toward hatching and that this increase was impaired by hypoxic incubation. Similarly, developmental increases in VEGF have been demonstrated in lamb intestine ${ }^{53}$, suggesting a role for VEGF in the development of the intestinal vascular network during fetal and early postnatal life. Although hypoxia is regarded as the most potent regulator of $\mathrm{VEGF}^{21}$, we did not find a higher VEGF expression in the intestine from the hypoxic embryos. In addition, when the hypoxic embryos were exposed to normoxia for the two last days of incubation, they did not undergo the posthatch increase in VEGF expression observed in the normoxic embryos. It can be speculated that the rapid intestinal growth that takes place between 19 and 21 days of incubation induces relative tissular hypoxia leading to 
increased VEGF expression. Embryos incubated in low $\mathrm{O}_{2}$ increase carrying capacity through increases in red blood cells, hemoglobin mass, or blood volume. ${ }^{33}$ Such adaptations to low $\mathrm{O}_{2}$ may have reduced the physiological hypoxia induced by rapid intestinal growth and, consequently, the intestinal expression of VEGF.

\section{FUTURE PERSPECTIVES}

Acute or chronic hypoxic injury to the gastrointestinal tract is believed to be a major contributing and potentially inciting factor in several neonatal conditions, including NEC. The series of experiments reported here present evidence that chronic moderate hypoxia during incubation results in subtle but significant alterations in chicken MA reactivity, small intestine morphology and VEGF expression. Whether these slight alterations may have a direct effect on the functional status of chick intestine remains uncertain. It can be speculated that the modest degree of the alterations induced by hypoxia is the result of the adaption of the embryo to the prolonged exposure to a relatively moderate insult. Critical time windows exist during development, and if environmental changes are experienced in the window of vulnerability, then the trajectory of development of the responding organ may be changed in ways that result in transient or persistent alterations. ${ }^{52}$ Therefore, new experiments will have to be conducted where embryos are exposed to different degrees of hypoxia across different temporal windows. 


\section{REFERENCES}

1 Bernstein, I. M., Horbar, J. D., Badger, G. J., Ohlsson, A. \& Golan, A. Morbidity and mortality among very-low-birth-weight neonates with intrauterine growth restriction. The Vermont Oxford Network. American journal of obstetrics and gynecology 182, 198-206 (2000).

2 Baserga, M. et al. Uteroplacental insufficiency decreases small intestine growth and alters apoptotic homeostasis in term intrauterine growth retarded rats. Early human development 79, 93-105 (2004).

3 Mickiewicz, M. et al. Structural and functional development of small intestine in intrauterine growth retarded porcine offspring born to gilts fed diets with differing protein ratios throughout pregnancy. Journal of physiology and pharmacology : an official journal of the Polish Physiological Society 63, 225-239 (2012).

4 Giussani, D. A., Salinas, C. E., Villena, M. \& Blanco, C. E. The role of oxygen in prenatal growth: studies in the chick embryo. The Journal of physiology 585 , 911-917 (2007).

5 Carlo, W. F., Villamor, E., Ambalavanan, N., DeMey, J. G. \& Blanco, C. E. Chronic exposure to cigarette smoke extract impairs endothelium-dependent relaxation of chicken embryo pulmonary arteries. Biology of the neonate $\mathbf{8 0}$, 247-250 (2001).

6 Villamor, E., Kessels, C. G., van Suylen, R. J., De Mey, J. G. \& Blanco, C. E. Cardiopulmonary effects of chronic administration of the NO synthase inhibitor L-NAME in the chick embryo. Biol Neonate 88, 156-163 (2005).

7 Ruijtenbeek, K., Kessels, L. C., De Mey, J. G. \& Blanco, C. E. Chronic moderate hypoxia and protein malnutrition both induce growth retardation, but have distinct effects on arterial endothelium-dependent reactivity in the chicken embryo. Pediatr Res 53, 573-579 (2003).

8 Villamor, E. et al. Chronic in ovo hypoxia decreases pulmonary arterial contractile reactivity and induces biventricular cardiac enlargement in the chicken embryo. Am J Physiol Regul Integr Comp Physiol 287, R642-651 (2004).

9 Lindgren, I., Zoer, B., Altimiras, J. \& Villamor, E. Reactivity of chicken chorioallantoic arteries, avian homologue of human fetoplacental arteries. 
Journal of physiology and pharmacology : an official journal of the Polish Physiological Society 61, 619-628 (2010).

10 Zoer, B. et al. Effects of prenatal hypoxia on pulmonary vascular reactivity in chickens prone to pulmonary hypertension. J Physiol Pharmacol 60, 119-130 (2009).

11 Agren, P. et al. Developmental changes in endothelium-dependent relaxation of the chicken ductus arteriosus. J Physiol Pharmacol 59, 55-76 (2008).

12 Marino, M. et al. Perinatal hypoxia triggers alterations in $\mathrm{K}+$ channels of adult pulmonary artery smooth muscle cells. American journal of physiology 293, L1171-1182 (2007).

13 Herrera, E. A. et al. High-altitude chronic hypoxia during gestation and after birth modifies cardiovascular responses in newborn sheep. Am J Physiol Regul Integr Comp Physiol 292, R2234-2240 (2007).

14 Ruijtenbeek, K. et al. Chronic hypoxia stimulates periarterial sympathetic nerve development in chicken embryo. Circulation 102, 2892-2897 (2000).

15 Villamor, E. et al. Chronic in ovo hypoxia decreases pulmonary arterial contractile reactivity and induces biventricular cardiac enlargement in the chicken embryo. Am J Physiol Regul Integr Comp Physiol 287, R642-R651 (2004).

16 Been, J. V. et al. Pulmonary vascular endothelial growth factor expression and disaturated phospholipid content in a chicken model of hypoxia-induced fetal growth restriction. Neonatology 97: 183-189 (2009).

17 Dzialowski, E. M., von Plettenberg, D., Elmonoufy, N. A. \& Burggren, W. W. Chronic hypoxia alters the physiological and morphological trajectories of developing chicken embryos. Comp Biochem Physiol A Mol Integr Physiol 131, 713-724 (2002).

18 Tintu, A. N., Noble, F. A. \& Rouwet, E. V. Hypoxia disturbs fetal hemodynamics and growth. Endothelium 14, 353-360 (2007).

19 Ruijtenbeek, K. et al. Chronic moderate hypoxia during in ovo development alters arterial reactivity in chickens. Pflugers Arch 447, 158-167 (2003).

20 Van der Sterren, S. et al. Morphological and functional alterations of the ductus arteriosus in a chicken model of hypoxia-induced fetal growth retardation. Pediatr Res 65, 279-284 (2009). 
21 Neufeld, G., Cohen, T., Gengrinovitch, S. \& Poltorak, Z. Vascular endothelial growth factor (VEGF) and its receptors. The FASEB journal : official publication of the Federation of American Societies for Experimental Biology 13, 9-22 (1999).

22 Banyasz, I. et al. Genetic polymorphisms for vascular endothelial growth factor in perinatal complications. Eur Cytokine Netw 17, 266-270 (2006).

23 Moonen, R. M. \& Villamor, E. Developmental changes in mesenteric artery reactivity in embryonic and newly hatched chicks. J Comp Physiol B 181 (2011).

24 Levinsohn, E. M., Packard, D. S., Jr., West, E. M. \& Hootnick, D. R. Arterial anatomy of chicken embryo and hatchling. Am J Anat 169, 377-405 (1984).

25 Altimiras, J. \& Crossley, D. A., 2nd. Control of blood pressure mediated by baroreflex changes of heart rate in the chicken embryo (Gallus gallus). Am J Physiol Regul Integr Comp Physiol 278, R980-986 (2000).

26 Uni, Z., Tako, E., Gal-Garber, O. \& Sklan, D. Morphological, molecular, and functional changes in the chicken small intestine of the late-term embryo. Poult Sci 82, 1747-1754 (2003).

27 Sugishita, Y. et al. Expression of genes encoding vascular endothelial growth factor and its Flk-1 receptor in the chick embryonic heart. J Mol Cell Cardio/32, 1039-1051 (2000).

28 Tischer, E. et al. The human gene for vascular endothelial growth factor. Multiple protein forms are encoded through alternative exon splicing. $J$ Biol Chem 266, 11947-11954 (1991).

29 Flamme, I., von Reutern, M., Drexler, H. C., Syed-Ali, S. \& Risau, W. Overexpression of vascular endothelial growth factor in the avian embryo induces hypervascularization and increased vascular permeability without alterations of embryonic pattern formation. Developmental biology 171 (1995).

30 Zabielski, R., Godlewski, M. M. \& Guilloteau, P. Control of development of gastrointestinal system in neonates. Journal of physiology and pharmacology : an official journal of the Polish Physiological Society 59 Suppl 1, 35-54 (2008).

31 Johnston, S. D., Orgeig, S., Lopatko, O. V. \& Daniels, C. B. Development of the pulmonary surfactant system in two oviparous vertebrates. Am J Physiol Regul Integr Comp Physiol 278, R486-493 (2000). 
32 Rahn, H., Paganelli, C. V. \& Ar, A. The avian egg: air-cell gas tension, metabolism and incubation time. Respiration physiology 22, 297-309 (1974).

33 Molenaar, R. et al. Effect of eggshell temperature and oxygen concentration on survival rate and nutrient utilization in chicken embryos. Poult Sci 89 (2010).

34 Williams, S. J., Campbell, M. E., McMillen, I. C. \& Davidge, S. T. Differential effects of maternal hypoxia or nutrient restriction on carotid and femoral vascular function in neonatal rats. Am J Physiol Regul Integr Comp Physiol 288, R360-367 (2005).

35 Lindgren, I., Crossley, D., 2nd, Villamor, E. \& Altimiras, J. Hypotension in the chronically hypoxic chicken embryo is related to the beta-adrenergic response of chorioallantoic and femoral arteries and not to bradycardia. Am J Physiol Regul Integr Comp Physio/ 301, R1161-1168 (2011).

36 Reber, K. M., Nankervis, C. A. \& Nowicki, P. T. Newborn intestinal circulation. Physiology and pathophysiology. Clin Perinatol 29, 23-39 (2002).

37 Ostlund, E., Lindholm, H., Hemsen, A. \& Fried, G. Fetal erythropoietin and endothelin-1: relation to hypoxia and intrauterine growth retardation. Acta obstetricia et gynecologica Scandinavica 79, 276-282 (2000).

38 Gao, Y. \& Raj, J. U. Regulation of the pulmonary circulation in the fetus and newborn. Physiological reviews 90, 1291-1335 (2010).

39 Villamor, E., Ruijtenbeek, K., Pulgar, V., De Mey, J. G. \& Blanco, C. E. Vascular reactivity in intrapulmonary arteries of chicken embryos during transition to ex ovo life. Am J Physiol Regul Integr Comp Physiol 282, R917-927 (2002).

40 Agren, P. et al. Ontogeny of chicken ductus arteriosus response to oxygen and vasoconstrictors. Am J Physiol Regul Integr Comp Physiol 292, R485-496 (2007).

41 Eddahibi, S. et al. Dilator effect of endothelins in pulmonary circulation: changes associated with chronic hypoxia. The American journal of physiology 265, L571580 (1993).

42 Noguchi, Y., Hislop, A. A. \& Haworth, S. G. Influence of hypoxia on endothelin1 binding sites in neonatal porcine pulmonary vasculature. The American journal of physiology 272, H669-678 (1997). 
43 le Noble, F. A., Ruijtenbeek, K., Gommers, S., de Mey, J. G. \& Blanco, C. E. Contractile and relaxing reactivity in carotid and femoral arteries of chicken embryos. Am J Physiol Heart Circ Physiol 278, H1261-1268 (2000).

44 Schuurman, M. J. \& Villamor, E. Endothelium-dependent contraction induced by acetylcholine in the chicken ductus arteriosus involves cyclooxygenase-1 activation and TP receptor stimulation. Comp Biochem Physiol A Mol Integr Physiol 157, 28-34.

45 Brandes, R. P. et al. Increased nitrovasodilator sensitivity in endothelial nitric oxide synthase knockout mice: role of soluble guanylyl cyclase. Hypertension 35, 231-236 (2000).

46 Newcomer, S. C., Taylor, J. C., McAllister, R. M. \& Laughlin, M. H. Effects of chronic nitric oxide synthase inhibition on endothelium-dependent and independent relaxation in arteries that perfuse skeletal muscle of swine. Endothelium 15, 17-31 (2008).

47 Ortiz, P. A. \& Garvin, J. L. Cardiovascular and renal control in NOS-deficient mouse models. Am J Physiol Regul Integr Comp Physiol 284, R628-638 (2003).

48 Sabatakou, O., Paraskevakou, E., Tseleni-Balafouta, S., Athanasiadis, A. \& Fasseas, K. The development of the chicken small intestine: a scanning electron microscopy study. J Submicrosc Cytol Pathol 35, 323-330 (2003).

49 Uni, Z., Geyra, A., Ben-Hur, H. \& Sklan, D. Small intestinal development in the young chick: crypt formation and enterocyte proliferation and migration. Br Poult Sci 41, 544-551 (2000).

50 Geyra, A., Uni, Z. \& Sklan, D. Enterocyte dynamics and mucosal development in the posthatch chick. Poult Sci 80, 776-782 (2001).

51 Uni, Z., Smirnov, A. \& Sklan, D. Pre- and posthatch development of goblet cells in the broiler small intestine: effect of delayed access to feed. Poult Sci 82, 320327 (2003).

52 Tibboel, D., van Nie, C. J. \& Molenaar, J. C. The effects of temporary general hypoxia and local ischemia on the development of the intestines: an experimental study. Journal of pediatric surgery 15, 57-62 (1980).

53 Holmes, K. et al. Localization and control of expression of VEGF-A and the VEGFR-2 receptor in fetal sheep intestines. Pediatric research 63, 143-148 (2008). 
Chapter 4

HYPOXIA-INDUCED CONTRACTION OF CHICKEN EMBRYO MESENTERIC ARTERIES: MECHANISMS AND DEVELOPMENTAL CHANGES

Leonie Brinks, Rob M.J..Moonen, Javier Moral-Sanz, Bianca Barreira, Lilian Kessels,

Francisco Perez-Vizcaino, Angel Cogolludo, and Eduardo Villamor Am J Physiol Regul Integr Comp Physiol. 311, R858-R869 (2016)

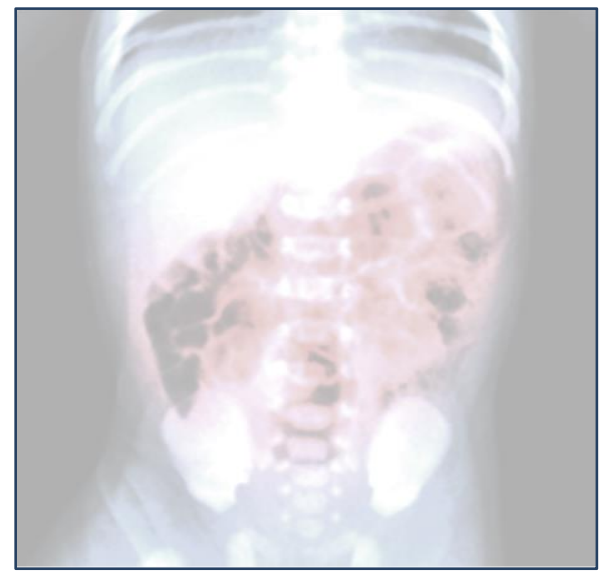




\section{ABSTRACT}

The fetal cardiovascular responses to acute hypoxia includes a redistribution of the cardiac output towards the heart and the brain at the expense of other organs, such as the intestine. We hypothesized that hypoxia exerts a direct effect on the mesenteric artery (MA) that may contribute to this response. Using wire myography, we investigated the response to hypoxia $\left(\mathrm{PO}_{2} \sim 2.5 \mathrm{kPa}\right.$ for $20 \mathrm{~min}$ ) of isolated MAs from 15- to 21-d chicken embryos (E15, E19 E21), and 1- to 45-d-old chickens (P1, P3, P14, $\mathrm{P} 45)$. Agonist-induced pretone or an intact endothelium were not required to obtain a consistent and reproducible response to hypoxia, which showed a pattern of initial rapid phasic contraction followed by a sustained tonic contraction. Phasic contraction was reduced by elimination of extracellular $\mathrm{Ca}^{2+}$ or by presence of the neurotoxin tetrodotoxin, the $\alpha_{1}$-adrenoceptor antagonist prazosin, or inhibitors of L-type voltagegated $\mathrm{Ca}^{2+}$ channels (nifedipine), mitochondrial electron transport chain (rotenone and antimycin A), and NADPH oxidase (VAS2870). The Rho-kinase inhibitor Y27632 impaired both phasic and tonic contraction and, when combined with elimination of extracellular $\mathrm{Ca}^{2+}$, hypoxia-induced contraction was virtually abolished. Hypoxic MA contraction was absent at E15 but present from E19 and increased towards the first days post-hatching. It then decreased during the first weeks of life and P45 MAs were unable to sustain hypoxia-induced contraction over time. In conclusion, the results of the present study demonstrate that hypoxic vasoconstriction is an intrinsic feature of chicken MA vascular smooth muscle cells during late embryogenesis and the perinatal period. 


\section{INTRODUCTION}

Oxygen tension is a key regulator of vascular tone. It is generally accepted that pulmonary arteries respond to regional hypoxia with vasoconstriction (hypoxic pulmonary vasoconstriction, HPV), whereas systemic arteries dilate. ${ }^{1-4}$ Systemic hypoxic vasodilation is a conserved physiological response to hypoxia that matches local blood flow and oxygen delivery to tissue metabolic demand. ${ }^{5}$ In the fetus, the cardiovascular responses to acute hypoxia includes a redistribution of the cardiac output away from the periphery towards high priority organs such as the heart, brain, and adrenal glands. ${ }^{6,7}$ This redistribution of blood flow occurs at the expense of other 'non-vital' organs (such as the intestine) and has long been suspected to be one of the major mechanisms responsible for ischemic injury and necrosis of the perinatal bowel. An importan component of hypoxia-induced flow redistribution is mediated by increased release of catecholamines ${ }^{8-11}$, whose effects are modulated by the different responsiveness of the vascular beds. Nevertheless, a direct effect of hypoxia and/or local actions of catecholamines on the mesenteric artery (MA) might contribute to the reduced flow in intestinal circulation.

Studies of the effects of hypoxia on isolated artery preparations eliminate potentially confounding effects of extravascular factors, such as circulating mediators, and allow evaluation of influences localized to the vessel wall. ${ }^{3}$ Hypoxia-induced contraction of isolated pulmonary ${ }^{12-14}$ and chorioallantoic ${ }^{12,15}$ arteries, hypoxic relaxation of isolated femoral arteries ${ }^{14,16}$ and normoxic contraction of isolated ductus arteriosus ${ }^{14,17-19}$ have been described in chicken embryos making this species an attractive model organism for the investigation of common mechanisms of vascular oxygen sensing and signaling. Recently, we characterized the developmental changes in MA reactivity from embryonic and newly hatched chicks. ${ }^{20,21}$ We observed that the ability of isolated chick MAs to respond to constrictor and dilator agonists increased during the embryonic period but no dramatic changes were induced by hatching or the first feeding. In the above study, the response of isolated MAs to hypoxia was not investigated. In the present study, we hypothesized that the chick MA is able to directly sense and respond to hypoxia and that the response may change with development. To test this hypothesis, we investigated the response to hypoxia of isolated MAs from embryonic, 
newly hatched, and juvenile chickens. We also hypothesized that the mechanisms involved in hypoxia sensing and signaling in MAs are similar to the ones described in pulmonary and systemic arteries of chicken embryos. To test this hypothesis, we analyzed the response to hypoxia of chicken embryo MAs in the presence of inhibitors of pathways proposed to participate in vascular oxygen signaling. 


\section{METHODS}

\section{INCUBATION OF CHICKEN (GALLUS GALLUS) EMBRYOS AND POST-HATCH DEVELOPMENT.}

All experimental procedures were carried out in accordance with the Dutch and Spanish Laws on Animal Experimentation and the European Directive for the Protection of Vertebrate Animals Used for Experimental and Other Scientific Purposes (86/609/EU) and approved by the Committee on Animal Experimentation of the University of Maastricht and the Complutense University. Fertilized eggs from White Leghorn chickens were incubated at $37.8^{\circ} \mathrm{C}, 45 \%$ humidity, and atmospheric oxygen concentration and rotated once per hour over an angle of $90^{\circ}$ (Incubator model $25 \mathrm{HS}$, Masalles Comercial, Spain). Embryonic chickens were studied at day 15 (E15), 19 (E19), or 21 (E21) of the 21 days of incubation. E19 embryos were studied before internal pipping and in E21 embryos internal, but not external, pipping was present. Post-hatch chickens were studied at the age of 1 (P1), 3 (P3), 12-16 (P14), or 42-48 (P45) days. Post-hatch chicks were reared in a brooder unit under $23 \mathrm{~h} /$ day of continuous light and temperature was decreased $\left(1^{\circ} \mathrm{C}\right.$ every $\left.48 \mathrm{~h}\right)$ from $35^{\circ} \mathrm{C}$ at hatch. During the grow-out period chickens were provided ad libitum access to water and a standard starter diet. ${ }^{13}$

\section{DISSECTION OF VESSELS AND RECORDING OF ARTERIAL REACTIVITY}

On the experimental day, the animals were killed by decapitation, placed on the dorsal side on a Petri-dish coated with silicon and a midline laparotomy and sternotomy were performed. With the aid of a dissecting microscope, the cranial MA was carefully dissected free from surrounding tissue. The cranial MA is the vessel which supplies most of the chicken intestine and also the yolk sac during the embryonic period. ${ }^{22}$ Only the intestinal part of the artery was used in our study.

Two stainless steel wires (diameter $40 \mu \mathrm{m}$ ) were inserted into the lumen of the vessels, which were mounted as a 1.7-2 mm length ring segment between an isometric force transducer and a displacement device in a myograph (Danish Myo Technology $\mathrm{A} / \mathrm{S}$ model 610M, Aarhus, Denmark). The myograph organ bath (5 mL vol) was filled with Krebs-Ringer bicarbonate buffer (KRB, composition in mmol/L: $\mathrm{NaCl}, 118.5 ; \mathrm{KCl}, 4.75$; 
$\mathrm{MgSO}_{4} \cdot 7 \mathrm{H}_{2} \mathrm{O}, 1.2 ; \mathrm{KH}_{2} \mathrm{PO}_{4}, 1.2 ; \mathrm{NaHCO}_{3}, 25.0 ; \mathrm{CaCl}_{2}$, 2.5; glucose, 5.5) maintained at $39^{\circ} \mathrm{C}$ and aerated with $21 \% \mathrm{O}_{2}-74 \% \mathrm{~N}_{2}-5 \% \mathrm{CO}_{2}\left(\mathrm{PO}_{2} \sim 19.2 \mathrm{kPa}\right.$, measured with an ABL 510 blood gas analyzer, Radiometer Copenhagen, Denmark). After an equilibration period of $30 \mathrm{~min}$, the vessels were distended to their individual optimal diameter, which evoked a resting tension corresponding to a transmural pressure of $10 \mathrm{mmHg}$ (E15) $20 \mathrm{mmHg}$ (E19 and E21), $40 \mathrm{mmHg}$ (P1 and P3), $60 \mathrm{mmHg}$ (P12), and $100 \mathrm{mmHg}$ (P45). These pressures correspond to the mean arterial blood pressure reported in chicken at the corresponding age ${ }^{23}$ and elicited the highest contractile response to $\mathrm{KCl}$, as determined in pilot experiments. After $30 \mathrm{~min}$. of incubation under resting tension, a control reference contraction was elicited by raising the $\mathrm{K}^{+}$concentration of the buffer $(80 \mathrm{mmol} / \mathrm{L})$ in exchange for $\mathrm{Na}^{+}$.

\section{RESPONSE TO HYPOXIA}

The effects of hypoxia on wall tension were generally studied in quiescent (i.e. without pretone), endothelium-intact MA rings. However, some experiments were performed under pretone induced by norepinephrine ( $\mathrm{NE}, 10 \mu \mathrm{mol} / \mathrm{L})$ or $\mathrm{KCl}(62.5 \mathrm{mmol} / \mathrm{L})$ or in endothelium-denuded rings. The endothelium was removed with a human hair and removal was confirmed by the absence of relaxation when acetylcholine $(1 \mu \mathrm{mol} / \mathrm{L})$ was applied to rings contracted with NE $(10 \mu \mathrm{mol} / \mathrm{L})$. To induce hypoxia, the organ chambers were wrapped in cling film and the gas mixture aerating the organ bath was switched from $21 \% \mathrm{O}_{2}-74 \% \mathrm{~N}_{2}-5 \% \mathrm{CO}_{2}$ to $95 \% \mathrm{~N}_{2}-5 \% \mathrm{CO}_{2}\left(\mathrm{PO}_{2} \sim 2.5 \mathrm{kPa}\right)$. In some experiments, vessels were incubated under $5 \% \mathrm{O}_{2}\left(\mathrm{PO}_{2} \sim 6.9 \mathrm{kPa}\right)$, instead of $21 \% \mathrm{O}_{2}$, before the exposure to hypoxia. After $20 \mathrm{~min}$ of hypoxic exposure, the gas mixture was switched back to $21 \% \mathrm{O}_{2}-74 \% \mathrm{~N}_{2}-5 \% \mathrm{CO}_{2}$ and the preparations were washed with the KRB buffer and allowed to equilibrate for at least 40 minutes before initiating a second hypoxic challenge (Fig. 1a). Except where otherwise stated, the first contraction served as the control and experimental interventions were made during the second contraction. The following pharmacological tools were used: the nitric oxide (NO) synthase inhibitor $\mathrm{N} \omega$-Nitro-L-arginine methyl ester (L-NAME, $0.1 \mathrm{mmol} / \mathrm{L}$ ), the cyclooxygenase inhibitor indomethacin $(10 \mu \mathrm{mol} / \mathrm{L})$, the nerve ending blocker tetrodotoxin $(5 \mu \mathrm{mol} / \mathrm{L})$; the selective $\alpha_{1}$-adrenoceptor antagonist prazosin $(1 \mu \mathrm{mol} / \mathrm{L})$; the mitochondrial electron transport chain (mETC) inhibitors rotenone (complex I, 1 $\mu \mathrm{mol} / \mathrm{L}$ ) and antimycin A (complex III, $1 \mu \mathrm{mol} / \mathrm{L}$ ); the $\mathrm{NADPH}$-oxidase inhibitor 
VAS2870 $(10 \mu \mathrm{mol} / \mathrm{L})$; the superoxide scavenger polyethylene glycol-superoxide dismutase (SOD, $100 \mathrm{U} / \mathrm{mL}$ ); the $\mathrm{H}_{2} \mathrm{O}_{2}$ scavenger polyethylene glycol catalase $(100 \mathrm{U} / \mathrm{mL})$; the $\mathrm{K}_{v}$ channel inhibitor 4-aminopyridine (4-AP, $\left.1 \mathrm{mmol} / \mathrm{L}\right)$; the L-type voltage-operated $\mathrm{Ca}^{2+}$ channel blocker nifedipine $(1 \mu \mathrm{mol} / \mathrm{L})$; the sarcoplasmic reticulum $\mathrm{Ca}^{2+}$ ATPase (SERCA) inhibitor thapsigargin $(2 \mu \mathrm{mol} / \mathrm{L})$; the store operated $\mathrm{Ca}^{2+}$ channel (SOCC) inhibitor SKF 96365 (10 $\left.\mu \mathrm{mol} / \mathrm{L}\right)$; and the Rho kinase inhibitor Y27632 (1 $\mu \mathrm{mol} / \mathrm{L})$. These drugs were added $15 \mathrm{~min}$ before the second hypoxic challenge and maintained during the whole experiment. The dependency on extracellular $\mathrm{Ca}^{2+}$ of hypoxic MA contraction was examined by omission of $\mathrm{Ca}^{2+}$ from the KRB solution and addition of EGTA ( $1 \mathrm{mmol} / \mathrm{L})$. The studies on the mechanisms involved in hypoxic MA contraction were only performed in E19 embryos.

\section{ELECTROPHYSIOLOGICAL STUDIES}

Potassium currents were recorded in isolated E19 MA smooth muscle cells. For cell isolation, endothelium-denuded MAs were dissected into a nominally $\mathrm{Ca}^{2+}$-free physiological salt solution ( $\mathrm{Ca}^{2+}$-free PSS) of composition (in mmol/L): $\mathrm{NaCl} 130, \mathrm{KCl}$ $5, \mathrm{MgCl}_{2}$ 1.2, glucose 10, HEPES $10(\mathrm{pH} 7.3$ with $\mathrm{NaOH})$ and then transferred to a $\mathrm{Ca}^{2+}$-free PSS containing (in $\mathrm{mg} / \mathrm{mL}$ ) papain 1, dithiothreitol 0.8 and albumin 0.7 for 15-20 minutes. Cells were stored in $\mathrm{Ca}^{2+}$-free PSS $\left(4^{\circ} \mathrm{C}\right)$ and used within 8 hours. Membrane currents were recorded with an Axopatch 200B and a Digidata 1322A (Axon Instruments, Burlingame, CA, U.S.A) using the whole-cell configuration of the patch clamp technique. Currents were normalized for cell capacitance and expressed in $\mathrm{pA} / \mathrm{pF}$ as previously described..$^{5,7}$ For recording optimal Kv currents, cells were superfused with an external $\mathrm{Ca}^{2+}$-free PSS (see above) and a $\mathrm{Ca}^{2+}$-free pipette (internal) solution containing (mmol/L): $\mathrm{KCl} 110, \mathrm{MgCl}_{2}$ 1.2, $\mathrm{Na}_{2} \mathrm{ATP} 5$, HEPES 10, EGTA 10, pH adjusted to 7.3 with $\mathrm{KOH}$. Hypoxia $\left(\mathrm{PO}_{2} \sim 3.2 \mathrm{kPa}\right)$ was induced by bubbling the $\mathrm{Ca}^{2+}$-free PSS with $100 \% \mathrm{~N}_{2}$.

\section{GLYOXYLIC ACID FLUORESCENCE HISTOCHEMISTRY}

To demonstrate the presence of catecholamine-containing nerves, E19 and P45 vessels were stained with glyoxylic acid as previously described. ${ }^{18}$ Whole mount preparations of vascular rings were incubated, while in the organ bath, in $2 \%$ glyoxylic 
acid and $10 \%$ sucrose in phosphate buffer for 30 minutes at room temperature. After this, the rings were air-dried (30 min), stretched on a glass slide at $100^{\circ} \mathrm{C}$ for $4 \mathrm{~min}$, and enclosed with entellan and a coverslip. Glyoxylic-acid-induced fluorescence was visualized using fluorescence microscopy (objective Fluo 10×, Nikon Diaphot, BA 470DM 455 filter, Nikon FE2 camera).

\section{DRUGS AND SOLUTIONS}

Solutions containing a high concentration of $\mathrm{KCl}(62.5$ or $80 \mathrm{mmol} / \mathrm{L}$ ) were prepared by replacing part of the $\mathrm{NaCl}$ of the $\mathrm{KRB}$ buffer by an equimolar amount of $\mathrm{KCl}$. All drugs were obtained from Sigma (St. Louis, MO, USA). All drugs were dissolved initially in distilled deionized water (except rotenone, antimycin A, thapsigargin, and VAS2870 in DMSO, and indomethacin in ethanol) to prepare adequate stock solutions and further dilutions were also made in deionized water.

\section{DATA ANALYSIS}

Results are shown as mean $\pm S E$ of measurements in $n$ animals. Contractions are expressed in terms of active wall tension ( $\mathrm{mN} / \mathrm{mm}$, calculated as the force divided by twice the length of the segment) or as a percentage of the reference contraction to $\mathrm{KCl}$ (80 $\mathrm{mmol} / \mathrm{L}$ ) performed for each individual ring at the beginning of the experiment. Differences between the control hypoxic challenge and the hypoxic challenge in the presence of a pharmacological blocker were assessed by paired student t-test. Differences between endothelial-denuded and intact endothelium were assessed by unpaired student t-test. Differences in the response to hypoxia in the presence or absence of pretone or at different ages were assessed by one-way ANOVA followed by Bonferroni's post hoc t-test or by ANOVA for linear trends. Differences were considered significant at a $P<0.05$. All analyses were performed using GraphPad Prism (version 5.00 for Windows, GraphPad Software, San Diego, California, USA). 


\section{RESULTS}

\section{RESPONSE TO HYPOXIA OF CHICKEN EMBRYO MESENTERIC ARTERY}

An example of the contractile response of an E19 MA ring to two successive 20 min hypoxic challenges, in the absence of pretone, is shown in Fig. 1a. Hypoxia led to a rapidly developing contraction (from here on referred to as phasic contraction) which peaked within 2-4 min. Tension then fell, reaching a nadir within 8-10 min, after which it tended to increase gradually and become sustained. This second phase is from now on named tonic contraction. The amplitude of the phasic contraction was measured as the initial peak in tension development occurring within the first $4 \mathrm{~min}$, whereas the amplitude of the tonic contraction was taken to be the level of tension above baseline measured after 20 min of hypoxia, immediately before reoxygenation. When hypoxia was maintained beyond $20 \mathrm{~min}$, tonic contraction reached a plateau. After $1 \mathrm{~h}$ of hypoxia, active wall tension was $0.23 \pm 0.06 \mathrm{mN} / \mathrm{mm}(n=6, P=0.002)$ above the tension reached at $20 \mathrm{~min}$.

Hypoxia-induced contraction was reproducible, as there was no significant change either in the phasic or the tonic contraction in a second hypoxic challenge (Fig. 1b). We therefore generally used the first contraction in each artery as the control and initiated an intervention designed to examine the mechanism 20 min before the second hypoxic challenge. Hypoxia-induced contraction in E19 MAs was not significantly affected by switching the $\mathrm{O}_{2}$ concentration from 5 to $0 \%$ instead of from 21 to $0 \%$ (Fig. 1c). As shown in Figure 1d, the mechanical removal of the endothelium, the presence of the NO synthase inhibitor L-NAME, or the presence of the cyclooxygenase inhibitor indomethacin did not produce significant changes in the hypoxic contraction of E19 MA rings. Incubation with L-NAME induced a tonic contraction $(0.09 \pm 0.01 \mathrm{mN} / \mathrm{mm}$, $n=6, P<0.01$ vs. baseline). 
A

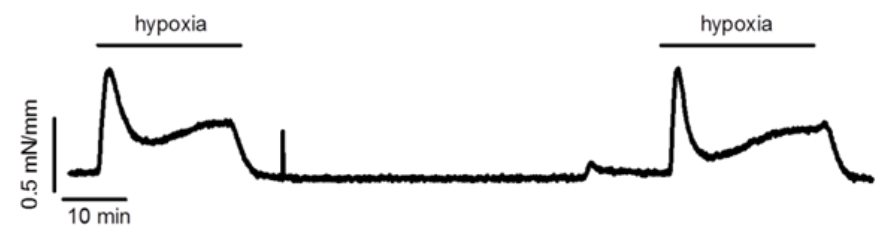

B

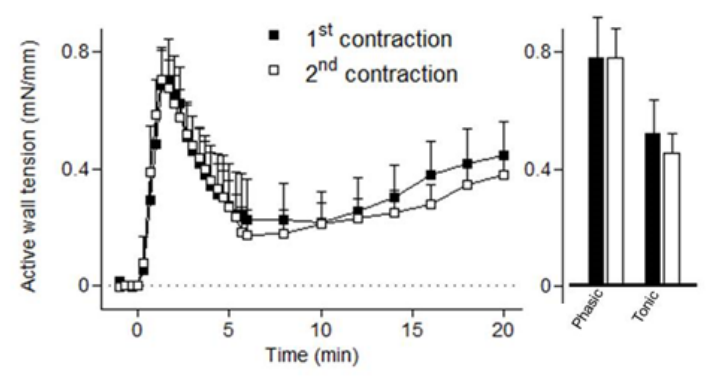

C

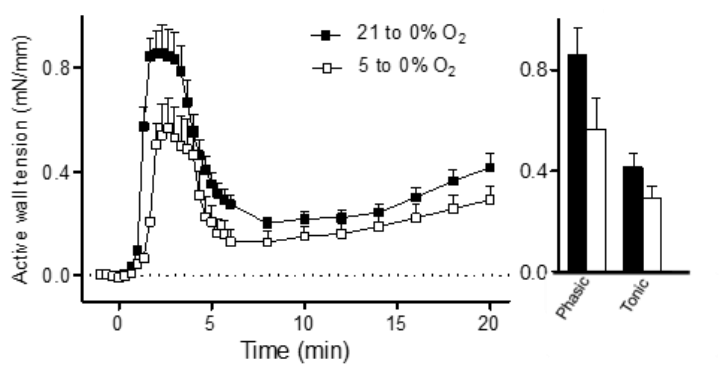

D

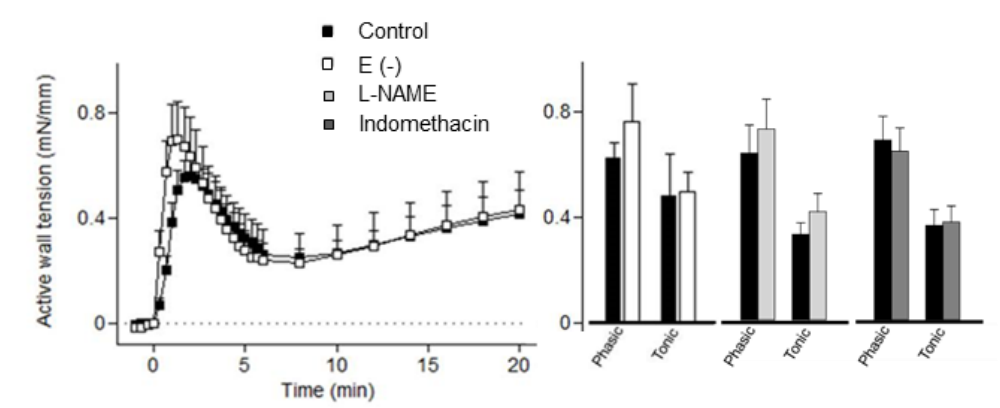

Figure 1. (A) Representative original tracing showing the response of an isolated E19 chicken mesenteric artery to two successive hypoxic challenges of 20 min duration. Hypoxia was induced by switching the gas mixture aerating the organ bath from $21 \% \mathrm{O}_{2}-74 \% \mathrm{~N}_{2}-5 \% \mathrm{CO}_{2}$ $\left(\mathrm{PO}_{2} \sim 19.2 \mathrm{kPa}\right)$ to $95 \% \mathrm{~N}_{2}-5 \% \mathrm{CO}_{2}\left(\mathrm{PO}_{2} \sim 2.5 \mathrm{kPa}\right)$. (B) Amplitude of hypoxic mesenteric contraction over 20 min during two successive exposures to hypoxia under control conditions 
$(E 19, n=8)$. (C) Response to hypoxia induced by switching the $\mathrm{O}_{2}$ concentration from 5 to $0 \%$ $(n=6)$ instead of from 21 to $0 \%(n=6)$. (D) Effects of endothelial denudation $(n=6)$, the nitric oxide synthase inhibitor L-NAME $(0.1 \mathrm{mmol} / \mathrm{L}, n=6)$, and the cyclooxygenase inhibitor indomethacin (10 $\mu \mathrm{mol} / \mathrm{L}, n=6)$ on the phasic and tonic contraction induced by hypoxia in $E 19$ chicken mesenteric arteries. The amplitude of the phasic contraction was measured as maximal tension developed within the first 4 min and the amplitude of the tonic contraction was taken to be the level of tension above baseline measured after 20 min of hypoxia. Results are shown as mean $\pm S E$ of measurements in $n$ embryos.

Figure 2 illustrates the effect of pretone induced by $\mathrm{NE}(10 \mu \mathrm{mol} / \mathrm{L})$ and $\mathrm{KCl}(62.5$ $\mathrm{mmol} / \mathrm{L}$ ) on the hypoxia-induced contraction of E19 MA rings. Neither the pattern (Fig. 2a) nor the amplitude (Fig. 2c) of the response were significantly affected by NEinduced pretone. In contrast, when pretone was elicited by $\mathrm{KCl}$ (Fig. 2b), an evident phasic peak was not observed and, after a rapid increase in tone, a slower but sustained contraction was developed. At the end of the 20 min hypoxic challenge, the tone developed by $\mathrm{KCl}$-contracted arteries was significantly higher than the one observed in the absence of pretone $(P=0.019)$ or under NE-induced pretone $(P=0.034)$ (Fig. 2c). 

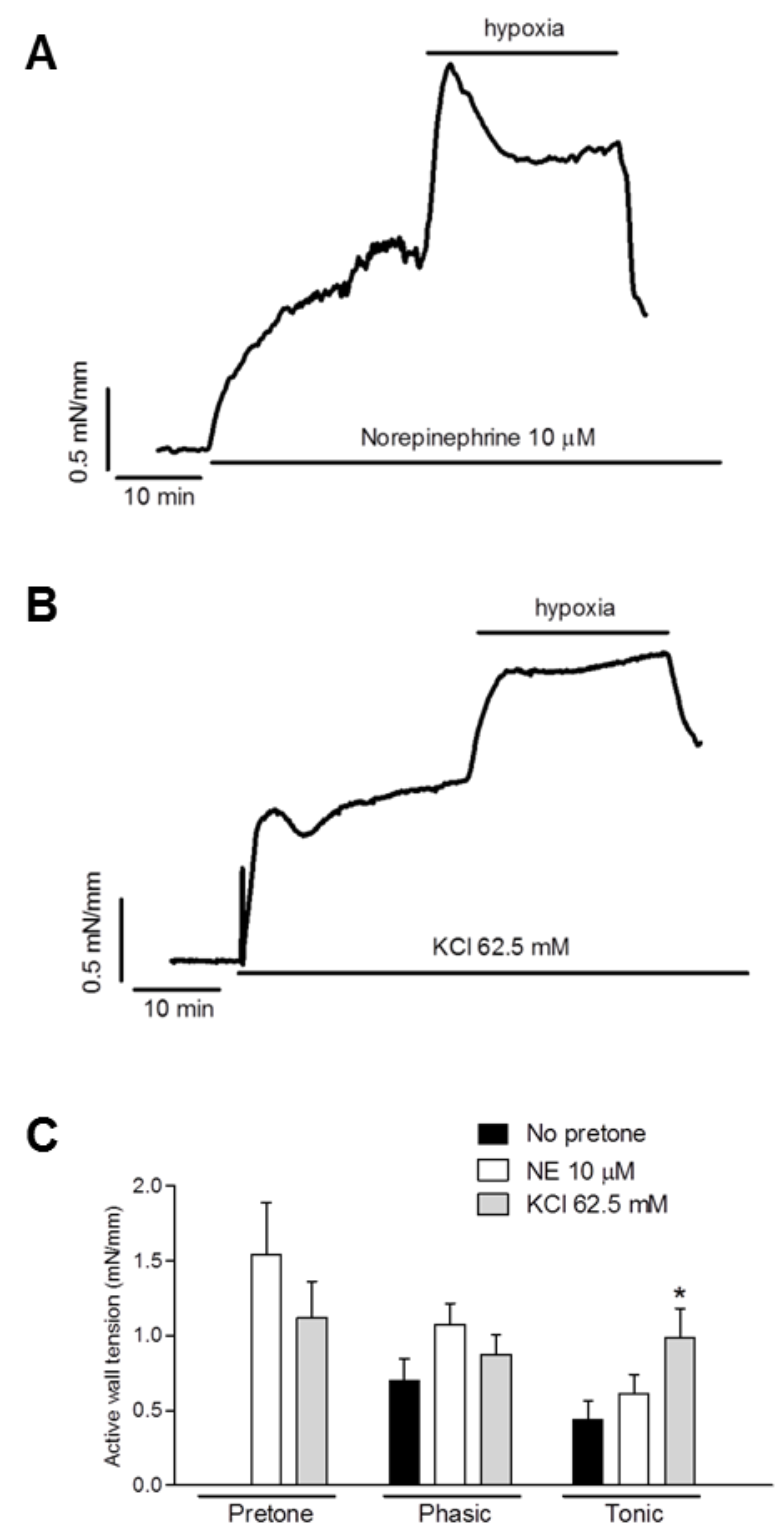

Figure 2. Representative original tracing showing the effects of pretone induced by (A) norepinephrine (NE, $10 \mu \mathrm{mol} / \mathrm{L})$ and $(B) \mathrm{KCl}(62.5 \mathrm{mmol} / \mathrm{L})$ on the response of isolated $\mathrm{E} 19$ chicken mesenteric arteries to a hypoxic challenge of 20 min duration. $C$. Effect of the absence $(n=6)$ or presence ( $n=6$ for each group) of pretone on the phasic and tonic contraction induced by hypoxia in E19 chicken mesenteric arteries. The amplitude of the phasic contraction was measured as maximal tension developed above pretone within the first 4 min and the amplitude of the tonic contraction was taken to be the level of tension above pretone measured after 20 min of hypoxia. Results are shown as mean $\pm S E$ of measurements in $n$ embryos. ${ }^{*} P<0.05$ vs. no pretone. 


\section{MECHANISMS INVOLVED IN HYPOXIA-INDUCED CONTRACTION OF CHICKEN EMBRYO MESENTERIC ARTERY}

As mentioned above, the response to hypoxia of E19 MA rings was endothelium independent (Fig. 1C). In order to assess the role of local release of catecholamines in the hypoxic response, we performed experiments in the presence of tetrodotoxin, a blocker of voltage-gated $\mathrm{Na}^{+}$channels in nerve cell membranes, and in the presence of the selective $\alpha_{1}$-adrenoceptor antagonist prazosin. As shown in Figures $3 a$ and $3 b$, tetrodotoxin and prazosin significantly impaired the phasic contraction $(P=0.025$ and $P=0.037$, respectively) but did not affect the tonic contraction. The presence of catecholamine containing nerves in chicken embryo mesenteric arteries was confirmed by glyoxylic acid fluorescence staining (Fig. 3c).

In order to test the role of the mETC and NADPH oxidase as sensors and reactive oxygen species (ROS) as mediators of the hypoxic response, we conducted experiments in the presence of the mETC inhibitors rotenone (Fig. 4a) and antimycin A (Fig. 4b), the NADPH-oxidase inhibitor VAS2870 (Fig. 4c), the superoxide scavenger SOD (Fig. 4d), or the $\mathrm{H}_{2} \mathrm{O}_{2}$ scavenger catalase (Fig. 4e). Rotenone $(P=0.036)$, antimycin $\mathrm{A}(P=0.009)$ and VAS2870 $(P=0.002)$ significantly impaired the phasic contraction to hypoxia.

In order to test the role of $\mathrm{Kv}$ channels as effectors of the hypoxic response, we conducted experiments in the presence of the Kv channel blocker 4-AP (Fig. 5a) and performed patch clamp experiments in E19 MA smooth muscle cells (Fig. $5 \mathrm{~b}$ and c). Incubation with 4-AP induced a tonic contraction $(0.64 \pm 0.11 \mathrm{mN} / \mathrm{mm}, \mathrm{n}=8, \mathrm{P}<0.01$ vs. baseline). The pretone induced by 4-AP did not significantly affect the pattern or the amplitude of the hypoxic response (Fig. $5 \mathrm{a}$ ). In the patch-clamp experiments, Kv currents were elicited by applying $200 \mathrm{~ms}$ depolarizing pulses from -60 to $+60 \mathrm{mV}$. As shown in Figures $5 b$ and $5 c$, exposure to hypoxia had no effect on Kv currents recorded in E19 MA smooth muscle cells. 


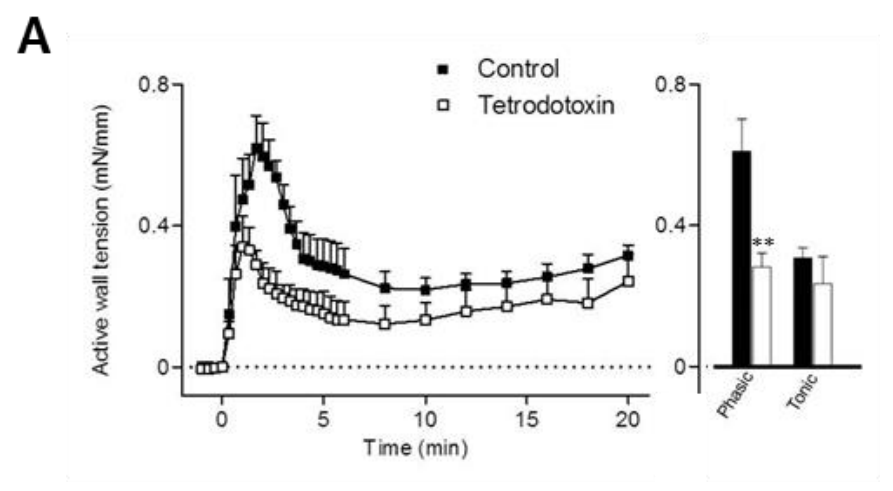

B

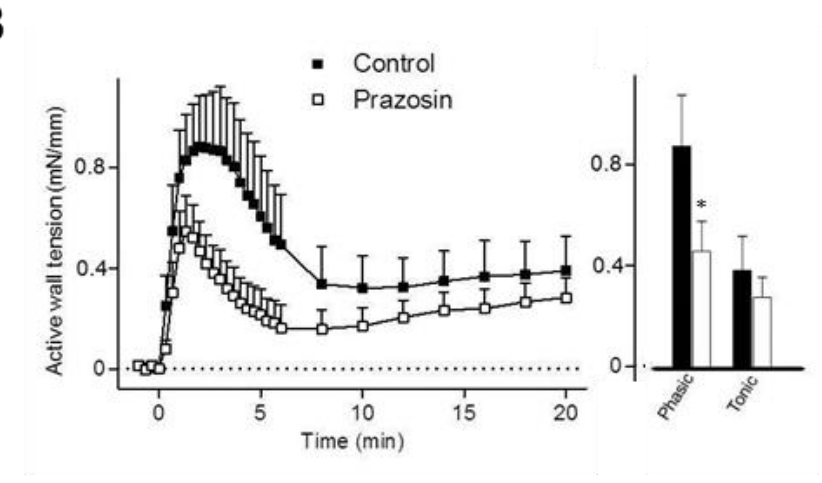

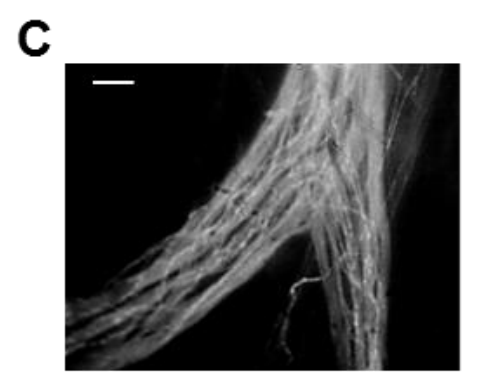

E19

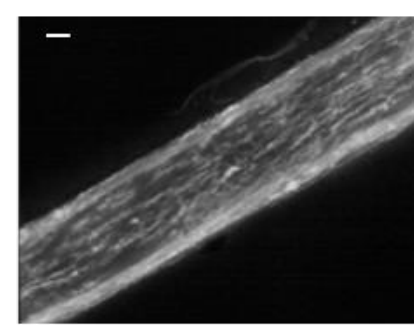

P45

Figure 3. Effects of the neurotoxin tetrodotoxin $(\boldsymbol{A}, 5 \mu \mathrm{mol} / \mathrm{L}, n=8)$ and the $\alpha 1$-adrenoceptor antagonist prazosin $(B, 1 \mu M, n=8)$ on the response of isolated $E 19$ chicken mesenteric arteries to two successive hypoxic challenges of 20 min duration. The first challenge (control) was performed in the absence of the drugs. Bars represent the phasic and tonic contraction induced by hypoxia in the two challenges. The amplitude of the phasic contraction was measured as maximal tension developed within the first 4 min and the amplitude of the tonic contraction was taken to be the level of tension above baseline measured after 20 min of hypoxia. Results are shown as mean $\pm S E$ of measurements in $n$ embryos. ${ }^{*} P<0.05$, ${ }^{* *} P<0.01$, first vs. second challenge. $\boldsymbol{C}$. Representative images of glyoxylic acid-induced fluorescence of catecholaminecontaining nerves in the mesenteric arteries from a 19-d chicken embryo (E19) and a 45-dold chicken (P45). Scale bar $200 \mu m$. 
A

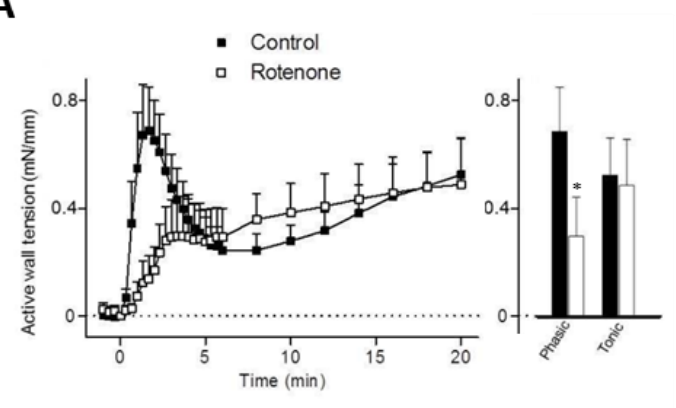

C

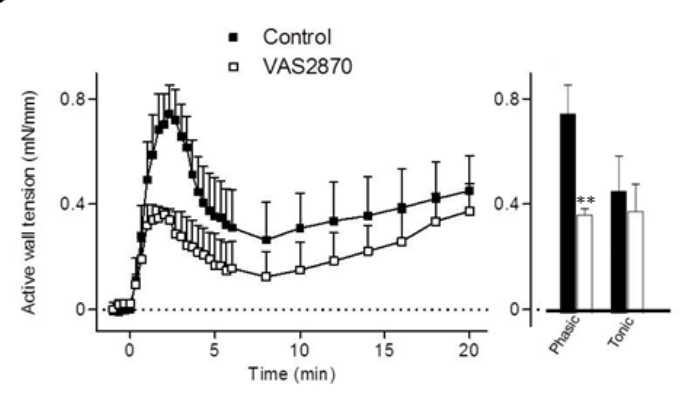

B

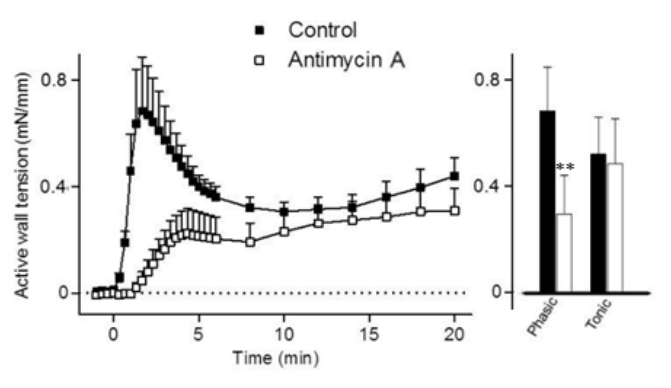

D

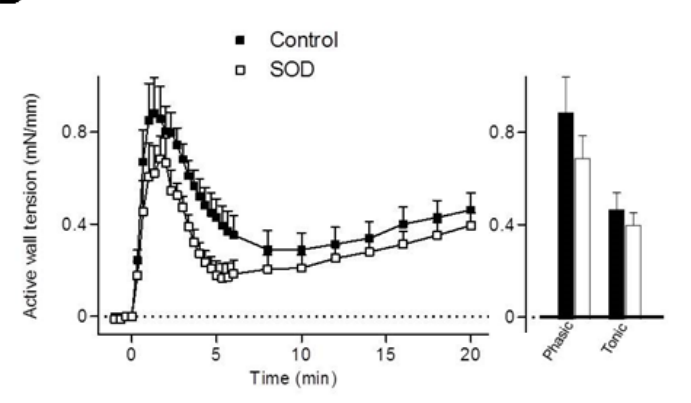

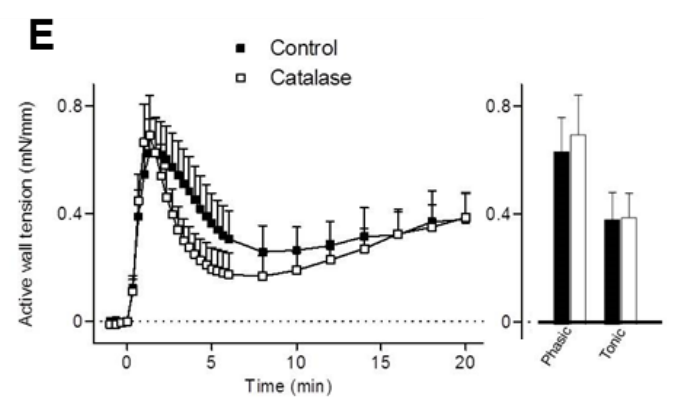

Figure 4. Effects of the presence of the mitochondrial electron transport chain inhibitors rotenone $(A, 1 \mu \mathrm{mol} / \mathrm{L}, n=8)$ and antimycin $A(B, 1 \mu \mathrm{mol} / \mathrm{L}, n=8)$, the NADPH oxidase inhibitor VAS2870 (C, $10 \mu \mathrm{mol} / \mathrm{L}, n=6)$, the superoxide scavenger polyethylene glycol superoxide dismutase ( $\boldsymbol{D}, \mathrm{SOD}, 100 \mathrm{U} / \mathrm{mL}, n=8)$, and the $\mathrm{H}_{2} \mathrm{O}_{2}$ scavenger polyethylene glycol catalase (E, $100 \mathrm{U} / \mathrm{mL}, n=8$ ) on the response of isolated E19 chicken mesenteric arteries to two successive hypoxic challenges of 20 min duration. The first challenge (control) was performed in the absence of the drugs. Bars represent the phasic and tonic contraction induced by hypoxia in the two challenges. The amplitude of the phasic contraction was measured as maximal tension developed within the first 4 min and the amplitude of the tonic contraction was taken to be the level of tension above baseline measured after 20 min of hypoxia. Results are shown as mean $\pm S E$ of measurements in $n$ embryos. ${ }^{*} P<0.05,{ }^{*} P<0.01$, first vs. second challenge. 
A

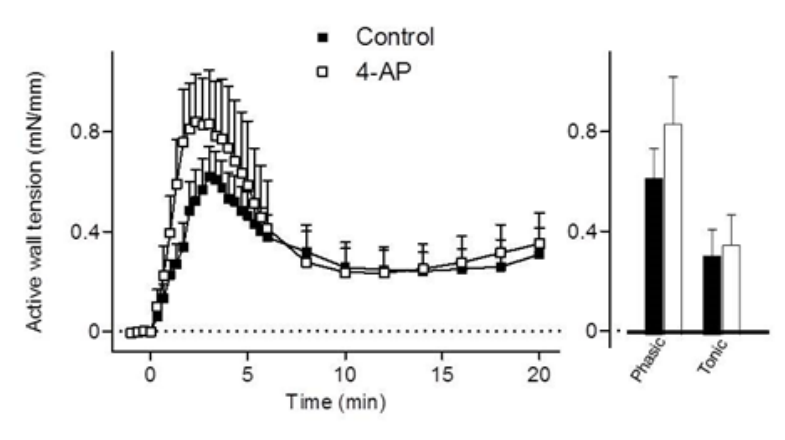

B

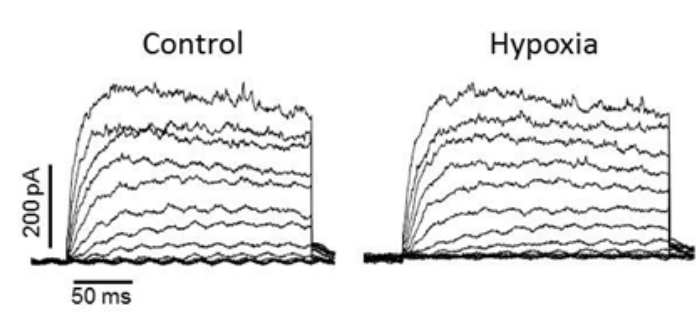

C

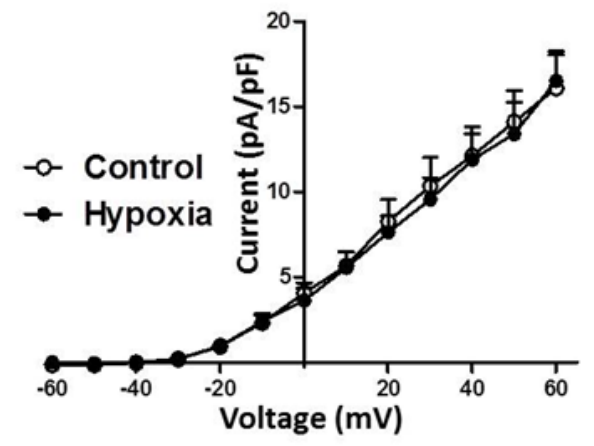

Figure 5. (A) Effects of the presence of the $K_{v}$ channel blocker 4-aminopyridine (4-AP, $\left.1 \mathrm{~mol} / \mathrm{L}\right)$ on the response of isolated E19 chicken mesenteric arteries to two successive hypoxic challenges of 20 min duration. The first challenge (control) was performed in the absence of the drugs. Bars represent the phasic and tonic contraction induced by hypoxia in the two challenges. The amplitude of the phasic contraction was measured as maximal tension developed within the first 4 min and the amplitude of the tonic contraction was taken to be the level of tension above baseline measured after 20 min of hypoxia. (B) Representative current traces for 200-ms depolarization pulses from $-60 \mathrm{mV}$ to $+60 \mathrm{mV}$ in $10 \mathrm{mV}$ increments from a holding potential $-60 \mathrm{mV}$ before (control) and after 5 minute exposition to hypoxia of an isolated smooth muscle cell from an E19 mesenteric artery. (C) Current-voltage relationships of $K_{V}$ currents measured at the end of the pulse before (control) and after the exposition to hypoxia of E19 mesenteric artery smooth muscle cells $(n=4)$. Results are shown as mean $\pm S E$ of measurements in $n$ embryos. 
Figure 6 summarizes the experiments aimed to analyze the role of $\mathrm{Ca}^{2+}$ channels and $\mathrm{Ca}^{2+}$ sensitization on the hypoxia-induced contraction of E19 MA rings. Omission of extracellular $\mathrm{Ca}^{2+}$ (Fig. 6a) or incubation of the vessels with the L-type voltageoperated $\mathrm{Ca}^{2+}$ channel inhibitor nifedipine (Fig. 6b) produced a significant impairment of the phasic contraction ( $P=0.019$ and $P=0.009$, respectively), whereas the SOCC blocker SKF 96365 did not affect hypoxic mesenteric contraction (Fig. 6c). Incubation of the vessels with the SERCA inhibitor thapsigargin elicited a tonic contraction (0.42 $\pm 0.09 \mathrm{mN} / \mathrm{mm}, \mathrm{n}=8)$. When thapsigargin-contracted MA rings were exposed to hypoxia, the peak of the phasic contraction was maintained but it was followed by a much more marked relaxation, which elicited a fall in wall tension below the basal tone (Fig. 6d). After this relaxation, tone underwent a slow recovery but an evident tonic contraction above the basal line was not observed (Fig. 6d). The role of Rho kinasemediated $\mathrm{Ca}^{2+}$ sensitization was tested by incubation with the Rho kinase inhibitor Y27632. As shown in Figure 6e, Y27632 impaired both phasic $(P=0.015)$ and tonic $(P=0.014)$ contractions. When the vessels were incubated with $Y 27632$ in a $\mathrm{Ca}^{2+}$-free medium, both phasic $(P<0.001)$ and tonic $(P=0.009)$ contraction were virtually abolished (Fig. 6f).

\section{DEVELOPMENTAL CHANGES IN HYPOXIA-INDUCED CONTRACTION OF CHICKEN MESENTERIC ARTERY}

As shown in Figure 7a, hypoxia did not induce contraction but decreased the resting tone in $\mathrm{E} 15 \mathrm{MA}$ rings $(-0.09 \pm 0.01 \mathrm{mN} / \mathrm{mm}, P<0.001 \mathrm{vs}$. baseline). A similar relaxation $(-0.07 \pm 0.01 \mathrm{mN} / \mathrm{mm})$ of $\mathrm{E} 15 \mathrm{MAs}$ was obtained by switching the $\mathrm{O}_{2}$ concentration from 5 to $0 \%$ instead of from 21 to $0 \%$. Hypoxia-induced contraction, which was observed at E19, increased towards the end of the incubation (i.e., E21, Fig. 7a) and was maintained in the first days post hatch (Fig. 7b-d). After the first postnatal days, both phasic and tonic contraction decreased and the P14 and P45 MA rings showed a delayed phasic contraction followed by a progressive decrease in tone (Fig. 7b). However, at the end of the 20 min hypoxic challenge, tone was still above the basal line. In contrast to the response to hypoxia, the contraction evoked by $\mathrm{KCl}(80 \mathrm{mM})$ underwent a marked developmental increase (Fig. 7e). 
A

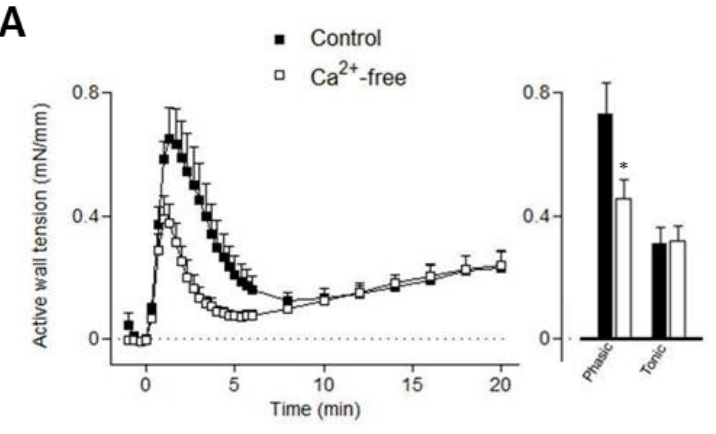

C

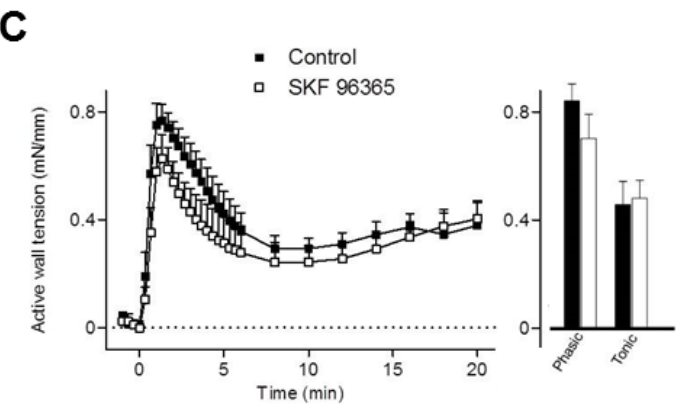

E

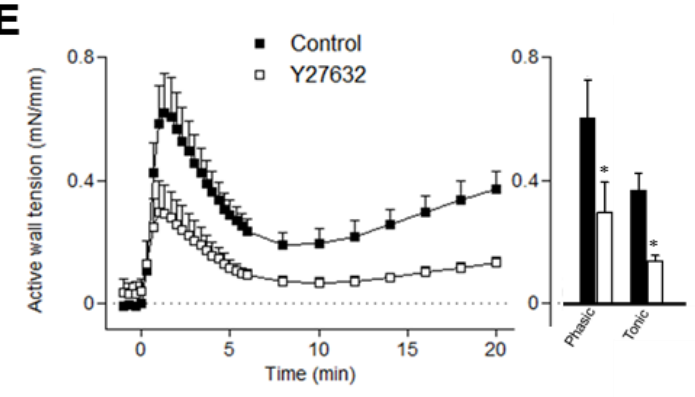

B

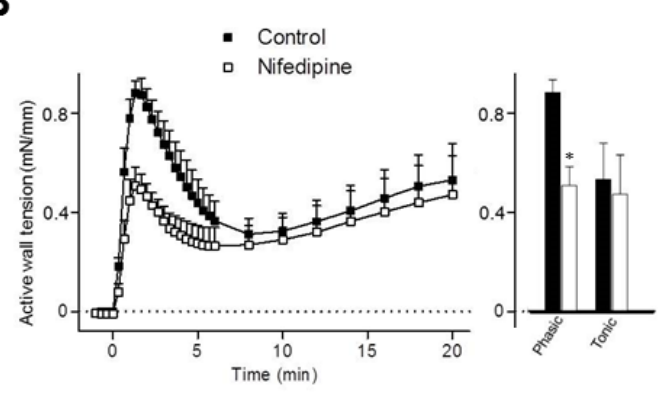

D

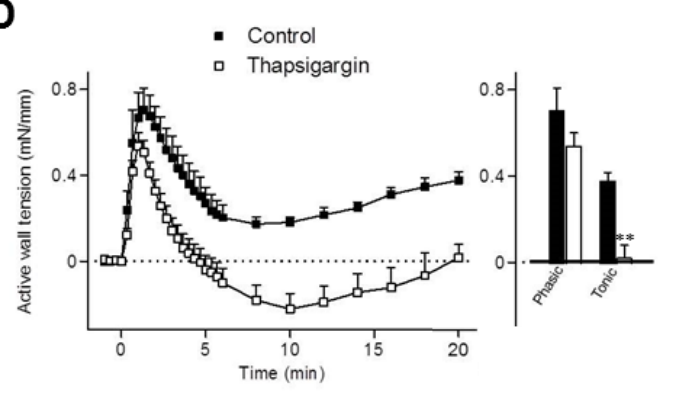

$\mathbf{F}$

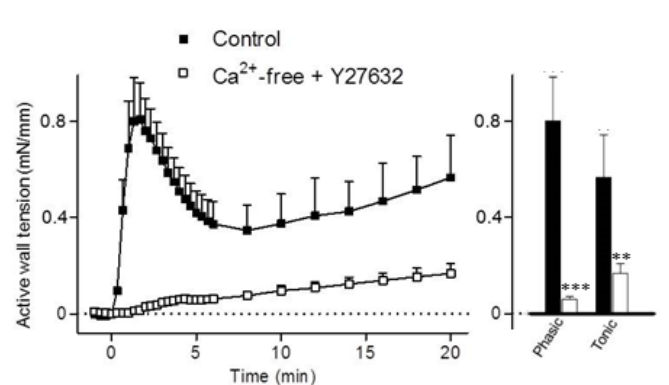

Figure 6. Effects of $\mathrm{Ca}^{2+}$-free solution containing $1 \mathrm{mmol} / \mathrm{L}$ EGTA $(\boldsymbol{A}, n=8)$, the presence of the L-type voltage-gated $\mathrm{Ca}^{2+}$ channel antagonist nifedipine $(\boldsymbol{B}, 1 \mu \mathrm{M}, n=8)$, the store operated $\mathrm{Ca}^{2+}$ channel blocker SKF 96365 (C, $10 \mu \mathrm{mol} / \mathrm{L}, n=8$ ), the sarcoplasmic reticulum $\mathrm{Ca}^{2+}$ ATPase inhibitor thapsigargin ( $\boldsymbol{D}, 2 \mu \mathrm{mol} / \mathrm{L}, n=8)$, the Rho kinase inhibitor Y27632 (E, $1 \mu \mathrm{mol} / \mathrm{L}, n=6$ ) and the combination $\mathrm{Ca}^{2+}$-free solution and $\mathrm{Y27632}(\boldsymbol{F}, n=6)$ on the response of isolated $\mathrm{E} 19$ chicken mesenteric arteries to two successive hypoxic challenges of 20 min duration. The first challenge (control) was performed in the absence of the drugs. Bars represent the phasic and tonic contraction induced by hypoxia in the two challenges. The amplitude of the phasic contraction was measured as maximal tension developed within the first 4 min and the amplitude of the tonic contraction was taken to be the level of tension above baseline measured after 20 min of hypoxia. Results are shown as mean $\pm S E$ of measurements in $n$ embryos. ${ }^{*} P<0.05,{ }^{* *} P<0.01,{ }^{* * *} P<0.001$, first vs. second challenge. 
A

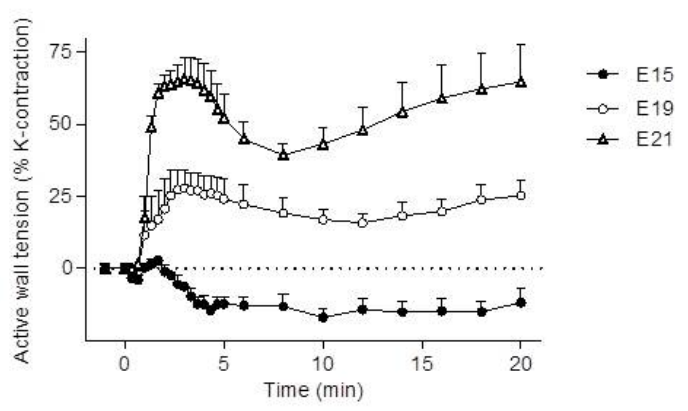

C

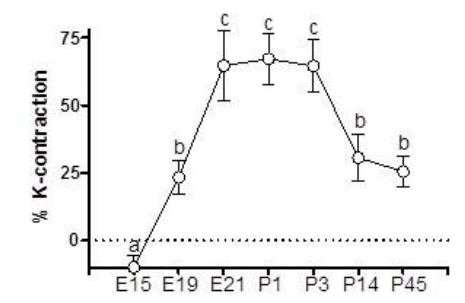

B

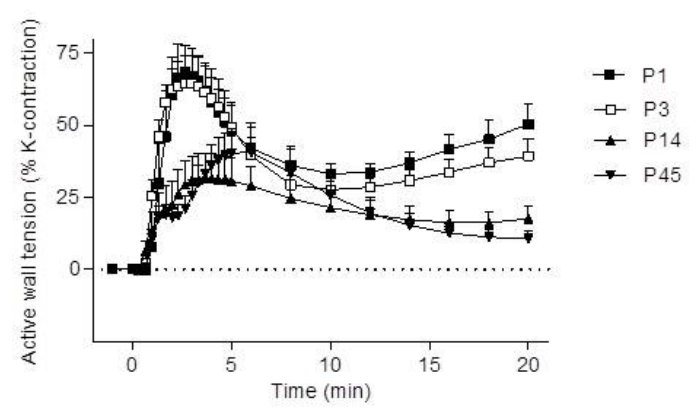

E

Figure 7. (A-D) Developmental changes on the response to 20 min of hypoxia of isolated mesenteric arteries from embryonic and post hatch chickens. Embryonic chickens were studied at day 15 (E15, $n=6), 19$ (E19, $n=10)$, or 21 (E21, $n=10)$ of the 21 days of incubation. Post-hatch chickens were studied at the age of 1 ( $P 1, n=10), 3(P 3, n=10), 12-16(P 14, n=10)$, or 42-48 ( $P 45, n=10)$ days. (C) Summary of the peak phasic contraction. (D) Summary of the tonic contraction at the end of the 20 min hypoxic challenge. (E) Developmental changes on the response to $\mathrm{KCl}(80 \mathrm{mmol} / \mathrm{L})$. The values are the tension achieved after $15 \mathrm{~min}$ of exposure to $\mathrm{KCl}$. All results are shown as mean $\pm S E$ of measurements in $n$ animals. Values in panels $C-E$ without a common letter are significantly different $(P<0.05)$. 


\section{DISCUSSION}

The results of the present study demonstrate that hypoxic vasoconstriction is an intrinsic feature of chicken MAs during late embryogenesis and the perinatal period. Agonist-induced pretone was not required to obtain a consistent and reproducible response, which showed a pattern of initial rapid phasic contraction followed by a sustained tonic contraction. The two phases demonstrated different properties. The phasic contraction was reduced by the elimination of extracellular $\mathrm{Ca}^{2+}$ or by the presence of the neurotoxin tetrodotoxin, the $\alpha_{1}$-adrenoceptor antagonist prazosin, or inhibitors of $\mathrm{L}$-type voltage-operated $\mathrm{Ca}^{2+}$ channels, $\mathrm{mETC}$, and NADPH oxidase. The tonic contraction was markedly impaired by the presence of the SERCA inhibitor thapsigargin. Rho-kinase inhibition impaired both phasic and tonic contraction and, when combined with elimination of extracellular $\mathrm{Ca}^{2+}$, hypoxia-induced contraction was virtually abolished. Ex vivo hypoxic MA contraction was present at E19 and increased towards the first days post-hatching. It then decreased during the first weeks of life.

\section{HYPOXIA-INDUCED CONTRACTION OF CHICKEN EMBRYO MESENTERIC} ARTERIES IN THE ABSENCE OF PRETONE

While some investigators were able to elicit contractile responses to hypoxia in isolated vessels under basal tone, most found that exposure of the tissues to agonist-induced preconstriction ('pretone') was required to obtain measurable and reproducible responses. ${ }^{3}$ In the case of isolated pulmonary arteries, pretone potentiates the hypoxia-induced contraction, whereas in systemic arteries pretone is necessary to obtain hypoxic relaxation. ${ }^{4,14,16}$ Nevertheless, pretone may confound the underlying mechanisms because there is an overlap between the constricting mechanisms activated by pretone agents and by hypoxia. ${ }^{24}$ In the present study, we observed that pretone was not necessary to obtain a consistent contraction in response to hypoxia in a systemic vessel, the chicken embryo MA. Moreover, NE-induced pretone did not significantly change the response to hypoxia and $\mathrm{KCl}$-induced pretone produced a modest increase of the tonic phase. As discussed below, this specific pattern of response of the MA to hypoxia suggests a relevant role of local vascular oxygen sensing and signaling in the systemic response to hypoxia. 


\section{ADRENERGIC CONTROL OF HYPOXIA-INDUCED CONTRACTION OF CHICKEN EMBRYO MESENTERIC ARTERY}

Since the embryonic period, chicken MAs are endowed with a dense network of catecholamine-containing nerves (Fig. $3 \mathrm{C}$ ) and are very sensitive to the effects of catecholamines. ${ }^{21}$ In the present study, we observed that the phasic hypoxia-induced contraction of E19 MA rings was impaired by the presence of the neurotoxin tetrodotoxin or the $\alpha$-1adrenoceptor antagonist prazosin. This suggests that local release of catecholamines contributes to this phase of hypoxic MA contraction. Accordingly, Borda et al. demonstrated that hypoxia evoked exocytotic release of norepinephrine in canine coronary arteries. ${ }^{25}$ Previous in vivo studies in the chicken embryo showed that systemic $\alpha$-adrenergic blockade with phentolamine impaired hypoxic mesenteric contraction ${ }^{26}$ and prevented the hypoxic redistribution of the cardiac output away from the intestine. ${ }^{9}$ As mentioned in the introduction, an important component of hypoxia-induced flow redistribution is mediated by increased release of catecholamines ${ }^{8-10}$, whose effects are modulated by the different responsiveness of the vascular beds. Previously, we observed that the chicken embryo MA is very sensitive to NE. ${ }^{21}$ In the present study, we contracted the E19 MA rings with a concentration of NE $(10 \mu \mathrm{mol} / \mathrm{L})$ that evoked approximately $90 \%$ of the maximal NEinduced contraction. ${ }^{21}$ Under these conditions, the response to hypoxia persisted and even the amplitude of the hypoxia-induced contraction was comparable with that of NE-induced contraction (Fig. 2c). In contrast, NE-contracted chicken embryo femoral arteries relaxed in response to hypoxia. ${ }^{14,16,27}$ Altogether, this suggests that, independently of catecholamines, the direct contractile effect of hypoxia in MAs may play a relevant role in the reduction of intestinal blood flow during acute hypoxia in the chicken embryo.

\section{REACTIVE OXYGEN SPECIES AND HYPOXIA-INDUCED CONTRACTION OF CHICKEN EMBRYO MESENTERIC ARTERIES}

ROS production, involving the mETC and NADPH oxidases, has been implicated as an important mechanism in vascular oxygen signalling. ${ }^{17,28-32}$ The primary ROS produced by mETC and NADPH oxidases is the superoxide radical. However, it is generally assumed that the highly reactive nature and short half-life of superoxide obviate its direct involvement as an autocrine or paracrine agent. Instead, it is believed 
that the nonradical and less reactive $\mathrm{H}_{2} \mathrm{O}_{2}$, formed by dismutation of superoxide by $\mathrm{SOD}$, is the actual signaling moiety. ${ }^{31,33} \mathrm{H}_{2} \mathrm{O}_{2}$ is degraded into water and molecular oxygen by catalase and peroxidases. ${ }^{31,33} \mathrm{~A}$ significant number of publications show that pharmacological inhibition of the mETC and/or NADPH oxidase, as well as superoxide and $\mathrm{H}_{2} \mathrm{O}_{2}$ scavenging, impaired HPV in several mammalian species. ${ }^{28-32,34}$ In chicken embryo vessels, mETC inhibitors impaired hypoxia-induced contraction of pulmonary arteries ${ }^{12}$, normoxic contraction of the ductus arteriosus ${ }^{17,19}$, and hypoxic relaxation of femoral arteries. ${ }^{16}$ Added to that, the presence of NADPH oxidase inhibitors or catalase impaired the response of chicken pulmonary arteries to hypoxia ${ }^{12}$ and ductus arteriosus to normoxia ${ }^{12}$ but did not alter hypoxic femoral relaxation. ${ }^{16}$ In the present study, we observed that both the blockade of mETC complexes I and III, and the blockade of NADPH oxidase induced a modest inhibition of the phasic but did not affect the tonic contraction evoked by hypoxia in chicken embryo MAs. Moreover, the presence of SOD or catalase did not significantly affect the response to hypoxia. Although not supported by actual measurements of ROS, our functional data do not suggest a major role for $\mathrm{mETC}$ and NADPH oxidase as sensors and ROS as mediators of the tonic phase of the contraction induced by hypoxia in chicken MAs. We speculate that the tonic phase has more pathophysiological relevance since it may lead to ischemia and necrosis of the gut.

In contrast to our results, investigations in mammalian models suggest the presence of an active vascular oxidant tone in the fetal circulation during late gestation. Antioxidants, via increased NO bioavailability, oppose ROS-induced contraction decreasing the magnitude of the peripheral vascular response to acute hypoxia. . $^{735-38}$ In the present study, we observed that incubation with the NO synthase inhibitor LNAME induced a tonic contraction in the chicken MAs, suggesting the presence of a basal production of $\mathrm{NO}$ in these vessels. However, hypoxia-induced contraction was not significantly affected by L-NAME or by removal of the endothelium, suggesting that differences in NO bioavailability do not contribute to the response. 


\section{$\mathrm{K}^{+}$AND $\mathrm{Ca}^{2+}$ CHANNELS IN HYPOXIA-INDUCED CONTRACTION OF CHICKEN EMBRYO MESENTERIC ARTERIES}

A rise of the intracellular $\mathrm{Ca}^{2+}$ concentration ([Ca $\left.\left.{ }^{2+}\right] \mathrm{i}\right)$ in vascular smooth muscle cells is necessary to initiate the $\mathrm{Ca}^{2+}$-calmodulin-mediated activation of myosin light chain kinase (MLCK), which in turn phosphorylates the myosin light chain, resulting in actinmyosin interaction and contraction. ${ }^{39}$ Several mechanisms can account for this rise in $\left[\mathrm{Ca}^{2+}\right] \mathrm{i}$ during hypoxia. In pulmonary arteries, the main proposed pathway is based on hypoxia-mediated inhibition of $\mathrm{Kv}$ channels, causing membrane depolarization that leads to $\mathrm{Ca}^{2+}$ influx through L-type voltage-operated $\mathrm{Ca}^{2+}$ channels. ${ }^{40}$ Likewise, inhibition of $\mathrm{Kv}$ channels has been shown to contribute to HPV and normoxic contraction of the ductus arteriosus both in chicken ${ }^{12,17}$ and mammals. ${ }^{1,41}$ In this study, we observed that when the MA rings were contracted with high $\mathrm{K}^{+}$depolarizing solution, which prevents outward movement of $\mathrm{K}^{+}$, the response to hypoxia was maintained and even augmented. In addition, the Kv blocker 4-AP induced a tonic contraction of chick mesenteric arteries, which indicates the presence of functional $\mathrm{KV}_{\mathrm{v}}$ channels in our preparation, but this contraction did not significantly affect the subsequent hypoxia-induced contraction. In contrast, 4-AP-mediated contraction abolished the contraction elicited by hypoxia in other isolated vessels, such as the rat pulmonary artery. ${ }^{42}$ Finally, our patch-clamp experiments showed that Kv currents from chicken mesenteric artery smooth muscle cells were not hypoxia-sensitive. Similar insensitivity to hypoxia has been reported for $\mathrm{Kv}$ currents present in MA from mammals. ${ }^{41,43}$ All these data strengthen the idea that the sensitivity of vascular $\mathrm{Kv}$ channels to hypoxia is restricted to some oxygen sensitive vascular tissues. ${ }^{12}$

Omission of extracellular $\mathrm{Ca}^{2+}$ or incubation of the vessels with the L-type voltageoperated $\mathrm{Ca}^{2+}$ channel inhibitor nifedipine produced a mild impairment of the phasic hypoxia-induced contraction. However, under these experimental conditions, MA rings were still significantly contracted by hypoxia and the tonic phase remained unchanged. Additional pathways for increasing $\left[\mathrm{Ca}^{2+}\right] \mathrm{i}$ could be capacitative $\mathrm{Ca}^{2+}$ entry through SOCC or $\mathrm{Ca}^{2+}$ release from sarcoplasmic reticulum stores. ${ }^{33,44,45}$ We observed that the presence of the SOCC blocker SKF 96365 did not affect hypoxic mesenteric contraction. In contrast, when the MAs were incubated with the SERCA inhibitor thapsigargin, the peak of the tonic contraction was maintained but it was followed by a much more marked relaxation, which elicited a fall in wall tension below the basal tone 
(Fig. 6d). After this relaxation, tone underwent a slow recovery but an evident tonic contraction was not observed. These results suggest that release of $\mathrm{Ca}^{2+}$ from the sarcoplasmic reticulum play a determinant role in the sustained contraction evoked by hypoxia in chicken embryo MAs.

$\mathrm{Ca}^{2+}$ sensitization of the myofilament due to Rho/Rho kinase-mediated MLC phosphatase inhibition is proposed as one of the main mechanisms of hypoxia-induced contraction in mammalian pulmonary arteries. ${ }^{33,46}$ In our experiments, the presence of the Rho-kinase inhibitor Y-27632 impaired both the phasic and the tonic contraction induced by hypoxia in E19 MA rings. Accordingly, Rho-kinase inhibition also impaired hypoxia-induced contraction of chicken embryo pulmonary arteries ${ }^{12}$ and normoxic contraction of chicken ductus arteriosus. ${ }^{17}$ Moreover, when Rho-kinase inhibition and removal of extracellular $\mathrm{Ca}^{2+}$ were combined both phases of hypoxic mesenteric contraction were virtually abolished. This suggests that the maintenance of the tonic response in the absence of extracellular $\mathrm{Ca}^{2+}$ may be possible through a Rho/Rhokinase-mediated $\mathrm{Ca}^{2+}$ sensitization. However, it should be noted that Wang et al. demonstrated that the Rho kinase inhibitors Y-27632 and ML-9 impaired the response to hypoxia of rat pulmonary arteries by preventing $\mathrm{Ca}^{2+}$ release from the sarcoplasmic reticulum. ${ }^{47}$ Our experimental approach did not include measurements of $\left[\mathrm{Ca}^{2+}\right] \mathrm{i}$ and therefore, do not allow us to draw any conclusion on whether a similar mechanism is present in hypoxic mesenteric contraction.

\section{DEVELOPMENTAL CHANGES IN HYPOXIA-INDUCED CONTRACTION OF CHICKEN MESENTERIC ARTERY}

We observed that hypoxia-induced contraction in isolated MAs from chickens was absent in the more immature embryos (i.e., E15) but present in the late embryonic period (i.e., E19). The response increased towards the first days post hatching, after which it decreased during the first weeks of life. Interestingly, the decrease with age in hypoxic mesenteric contraction resulted in a lack of ability of P45 arteries to sustain prolonged contraction (Fig. 7b). Therefore, our data suggest that hypoxia-induced contraction of isolated MAs is a distinctive feature of the late fetal and perinatal period. 
Although the E15 MA is responsive to several contractile agents, such as NE, endothelin-1, or thromboxane $\mathrm{A}_{2}{ }^{21}$, contraction to hypoxia was not present at this stage of development. Moreover, E15 MAs responded to hypoxia with relaxation. An alternative explanation for our findings could be that E15 vessels are already developing under relative hypoxia and, therefore, may be less susceptible to our experimental hypoxic conditions. In contrast to our results, Rouwet et al., using intravital microscopy, demonstrated that E13 and E17 second order chicken MAs responded to hypoxia with vasoconstriction. ${ }^{26}$ However, the experimental setup of Rouwet et al. reproduced global response to hypoxia with systemic release of catecholamines. ${ }^{26}$ Nevertheless, the response to hypoxia, under these conditions, also increased with development. ${ }^{26}$

To the best of our knowledge, no study has investigated the response to hypoxia in isolated fetal MAs and even the data in neonatal MAs are scarce. ${ }^{48-50}$ Nankervis et al. investigated the response to $15 \mathrm{~min}$ hypoxia of MA rings from 3- and 35-day-old swine. Pretone was induced with phenylephrine. They observed that rings from both age groups consistently demonstrated a triphasic response: brief relaxation/sharp contraction/sustained relaxation. Interestingly, the only part of the triphasic response that was age dependent was the contraction, which was significantly greater in rings from younger animals. ${ }^{48}$ However, the contraction was mediated by hypoxia-induced suppression of endothelium-derived NO production ${ }^{48}$, whereas in chicken MA rings the response to hypoxia was endothelium independent (Fig. $1 \mathrm{c}$ ). Another investigation dealing with postnatal changes in the local effects of hypoxia in MAs is the study of Nowicki et al., who perfused isolated loops of jejunum from 1-to 30-day-old swine with normoxic or hypoxic blood. ${ }^{49}$ They observed a slight vasodilation in the jejunal circulation in very young swine (i.e., $\leq 7$ days old) but marked vasodilation in the 14and 30-day old animals. In contrast isoproterenol-mediated vasodilatation did not change with development. ${ }^{49}$ Therefore, Nowicki et al. described a postnatal increase in hypoxia-induced mesenteric vasodilation, whereas Nankervis and the present study showed a postnatal decrease in hypoxic mesenteric vasoconstriction. Altogether these data suggest that, when compared with older vessels, the local response to hypoxia of perinatal mesenteric circulation does not facilitate intestinal perfusion. This 
circumstance may explain the increased propensity of newborn intestine to tissue damage following hypoxic-ischemic insults.

\section{SIGNIFICANCE AND PERSPECTIVES}

Necrotizing enterocolitis (NEC) is the most common gastrointestinal emergency in human very preterm neonates and is associated with significant short- and long-term morbidity and mortality. ${ }^{51,52}$ Although it is clear that intestinal ischemia plays a significant role in the pathogenesis of NEC, it is controversial whether this role is primary or secondary and what mechanisms lead to the ischemia. ${ }^{51,52}$ As reviewed by Young et al., epidemiologic evidence suggests that the most common form of NEC in very preterm infants is not triggered by a primary hypoxic-ischemic event. ${ }^{52}$ Moreover, the near-midgestation fetus shows attenuated vasomotor responses to severe asphyxia compared with more mature foetuses. ${ }^{53}$ In contrast, term infants with perinatal asphyxia or congenital heart disease who have development of intestinal necrosis, are more likely to have intestinal ischemia as a primary component of their disease pathogenesis. ${ }^{52}$ Our results showing that the local response to hypoxia was absent in the less mature MA, indirectly support the hypothesis that the immature intestine is less susceptible to direct hypoxic damage.

The search for a common ('universal') mechanism of vascular $\mathrm{O}_{2}$ sensing/signaling has occupied investigators for decades. ${ }^{2,3}$ However, although some of these mechanisms appear to be universally present, there are also important differences among species and vascular beds. Our study incorporates the MA to the list of vessels showing a specific response to hypoxia in the chicken embryo. ${ }^{12-19}$ We observed that, at least during the perinatal period, the chicken MA responds to hypoxia in a similar way than the pulmonary artery, i.e. with contraction. Moreover, our results show different levels of contribution to hypoxic MA contraction of the mechanisms involved in HPV. It has been proposed that hypoxic signaling in pulmonary artery smooth muscle cells is likely to occur in microdomains occupied by mitochondria, sarcoplasmic reticulum, and sarcolemma. ${ }^{24}$ Therefore, a complete understanding of the hypoxic response of pulmonary and systemic vessels will require accurate intracellular indicators of $\mathrm{ROS}$ and $\mathrm{Ca}^{2+}$ targeted to specific organelles. Ideally, such probes would distinguish among superoxide, $\mathrm{H}_{2} \mathrm{O}_{2}$, and other species, which might activate different 
Transduction pathways. ${ }^{24}$ In addition, alternative mechanisms of hypoxia sensing/signaling such as activation of AMP-activated protein kinase, stimulation of $\mathrm{Ca}^{2+}$-sensing receptors, production of hydrogen sulfide, alterations in $\mathrm{Na}^{+} / \mathrm{Ca}^{2+}$ exchange, inhibition of the breakdown of cyclic ADP ribose, or changes in the activity of heme oxygenase, cytochrome P-450 monooxygenases, and plasma membrane $\mathrm{Ca}^{2+-}$-ATPase $3,24,54,55$ remain to be investigated in MAs. Studies on the developmental changes of these pathways will contribute to a better understanding of the particular (patho)biology of blood vessels during the pre- and perinatal periods. Finally, the possible effects of chronic prenatal hypoxia ${ }^{13,20,56,57}$ on the MA response to acute hypoxia also warrant further investigation. 


\section{REFERENCES}

1 Weir, E., Lopez-Barneo, J., Buckler, K. \& SL, A. Acute oxygen-sensing mechanisms. New England Journal of Medicine. 353, 2042-2055 (2005).

2 Archer, S. \& Michelakis, E. The mechanism(s) of hypoxic pulmonary vasoconstriction: potassium channels, redox $\mathrm{O}(2)$ sensors, and controversies. News Physiol Sci 17, 131-137 (2002).

3 Sylvester, J. T., Shimoda, L. A., Aaronson, P. I. \& Ward, J. P. Hypoxic pulmonary vasoconstriction. Physiol Rev 92, 367-520 (2012).

4 Morales-Cano, D. et al. Kv7 channels critically determine coronary artery reactivity: left-right differences and down-regulation by hyperglycaemia. Cardiovasc Res 106, 98-108 (2015).

5 Maher, A. R. et al. Hypoxic modulation of exogenous nitrite-induced vasodilation in humans. Circulation 117, 670-677 (2008).

6 Giussani, D., Spencer, J., Moore, P., Bennet, L. \& Hanson, M. Afferent and efferent components of the cardiovascular reflex responses to acute hypoxia in term fetal sheep. The Journal of physiology 461, 431-449 (1993).

7 Giussani, D. A. The fetal brain sparing response to hypoxia: physiological mechanisms. The Journal of physiology 594:1215-1230 (2016).

8 Mulder, A. L., Miedema, A., De Mey, J. G., Giussani, D. A. \& Blanco, C. E. Sympathetic control of the cardiovascular response to acute hypoxemia in the chick embryo. Am J Physiol Regul Integr Comp Physiol 282, R1156-1163 (2002).

9 Mulder, A. L., van Goor, C. A., Giussani, D. A. \& Blanco, C. E. Alpha-adrenergic contribution to the cardiovascular response to acute hypoxemia in the chick embryo. Am J Physiol Regul Integr Comp Physiol 281, R2004-2010 (2001).

10 Mulder, A. L., Golde, J. M., Goor, A. A., Giussani, D. A. \& Blanco, C. E. Developmental changes in plasma catecholamine concentrations during normoxia and acute hypoxia in the chick embryo. The Journal of physiology 527 Pt 3, 593-599 (2000).

11 Fletcher, A. J., Gardner, D. S., Edwards, C. M. B., Fowden, A. L. \& Giussani, D. A. Development of the ovine fetal cardiovascular defense to hypoxemia towards 
full term. American Journal of Physiology-Heart and Circulatory Physiology 291, H3023-H3034 (2006).

12 Moreno, L. et al. Ceramide mediates acute oxygen sensing in vascular tissues. Antioxid Redox Signal 20, 1-14, doi:10.1089/ars.2012.4752 (2014).

13 Zoer, B. et al. Effects of prenatal hypoxia on pulmonary vascular reactivity in chickens prone to pulmonary hypertension. J Physiol Pharmacol 60, 119-130 (2009).

14 van der Sterren, S., Kleikers, P., Zimmermann, L. J. \& Villamor, E. Vasoactivity of the gasotransmitters hydrogen sulfide and carbon monoxide in the chicken ductus arteriosus. Am J Physiol Regul Integr Comp Physiol 301, R1186-1198 (2011).

15 Lindgren, I., Zoer, B., Altimiras, J. \& Villamor, E. Reactivity of chicken chorioallantoic arteries, avian homologue of human fetoplacental arteries. $J$ Physiol Pharmacol 61, 619-628 (2010).

16 Zoer, B. et al. Hypoxia sensing in the fetal chicken femoral artery is mediated by the mitochondrial electron transport chain. Am J Physiol Regul Integr Comp Physiol 298, R1026-1034 (2010).

17 Cogolludo, A. L. et al. Maturation of $\mathrm{O} 2$ sensing and signaling in the chicken ductus arteriosus. Am J Physiol Lung Cell Mol Physiol 297, L619-630 (2009).

18 Agren, P. et al. Ontogeny of chicken ductus arteriosus response to oxygen and vasoconstrictors. Am J Physiol Regul Integr Comp Physiol 292, R485-496 (2007).

19 Greyner, H. \& Dzialowski, E. M. Mechanisms mediating the oxygen-induced vasoreactivity of the ductus arteriosus in the chicken embryo. Am J Physiol Regul Integr Comp Physiol 295, R1647-1659 (2008).

20 Moonen, R. M., Kessels, C. G., Zimmermann, L. J. \& Villamor, E. Mesenteric artery reactivity and small intestine morphology in a chicken model of hypoxiainduced fetal growth restriction. J Physiol Pharmacol 63, 601-612 (2012).

21 Moonen, R. M. \& Villamor, E. Developmental changes in mesenteric artery reactivity in embryonic and newly hatched chicks. J Comp Physiol B 181:10631073 (2011). 
22 Mohammed, R., Cavallaro, G., Kessels, C. G. \& Villamor, E. Functional differences between the arteries perfusing gas exchange and nutritional membranes in the late chicken embryo. J Comp Physiol B 185:783-796 (2015).

23 Girard, H. Arterial pressure in the chick embryo. Am J Physiol 224, 454-460 (1973).

24 Connolly, M. J., Prieto-Lloret, J., Becker, S., Ward, J. P. \& Aaronson, P. I. Hypoxic pulmonary vasoconstriction in the absence of pretone: essential role for intracellular Ca2+ release. The Journal of physiology 591, 4473-4498 (2013).

25 Borda, L. J., Shuchleib, R. \& Henry, P. D. Hypoxic contraction of isolated canine coronary artery. Mediation by potassium-dependent exocytosis of norepinephrine. Circulation research 46, 870-879 (1980).

26 Rouwet, E. V. et al. Development of vasomotor responses in fetal mesenteric arteries. American journal of physiology 279, H1097-1105 (2000).

27 Ruijtenbeek, K., Kessels, C. G., Villamor, E., Blanco, C. E. \& De Mey, J. G. Direct effects of acute hypoxia on the reactivity of peripheral arteries of the chicken embryo. Am J Physiol Regul Integr Comp Physiol 283, R331-338 (2002).

28 Ward, J. P. T. Oxygen sensors in context. Biochimica et Biophysica Acta (BBA) - Bioenergetics 1777, 1-14 (2008).

29 Leach, R. M., Hill, H. M., Snetkov, V. A., Robertson, T. P. \& Ward, J. P. Divergent roles of glycolysis and the mitochondrial electron transport chain in hypoxic pulmonary vasoconstriction of the rat: identity of the hypoxic sensor. $J$ Physiol 536, 211-224 (2001).

30 Paddenberg, R. et al. Essential role of complex II of the respiratory chain in hypoxia-induced ROS generation in the pulmonary vasculature. Am J Physiol Lung Cell Mol Physiol 284, L710-719 (2003).

31 Cogolludo, A., Moreno, L. \& Villamor, E. Mechanisms controlling vascular tone in pulmonary arterial hypertension: implications for vasodilator therapy. Pharmacology 79, 65-75 (2007).

32 Frazziano, G. et al. Neutral sphingomyelinase, NADPH oxidase and reactive oxygen species. Role in acute hypoxic pulmonary vasoconstriction. J Cell Physiol 226:2633-2640 (2011). 
33 Aaronson, P. I. et al. Hypoxic pulmonary vasoconstriction: mechanisms and controversies. The Journal of physiology 570, 53-58 (2006).

34 Waypa, G. B. \& Schumacker, P. T. Hypoxic pulmonary vasoconstriction: redox events in oxygen sensing. J Appl Physiol 98, 404-414 (2005).

35 Thakor, A. et al. Redox modulation of the fetal cardiovascular defence to hypoxaemia. The Journal of physiology 588, 4235-4247 (2010).

36 Thakor, A. S. et al. Melatonin modulates the fetal cardiovascular defense response to acute hypoxia. Journal of pineal research 59, 80-90 (2015).

37 Kane, A. D. et al. Xanthine oxidase and the fetal cardiovascular defence to hypoxia in late gestation ovine pregnancy. The Journal of physiology $592,475-$ 489 (2014).

38 Kane, A. D., Herrera, E. A., Hansell, J. A. \& Giussani, D. A. Statin treatment depresses the fetal defence to acute hypoxia via increasing nitric oxide bioavailability. The Journal of physiology 590, 323-334 (2012).

39 Waypa, G. B. \& Schumacker, P. T. O(2) sensing in hypoxic pulmonary vasoconstriction: the mitochondrial door re-opens. Respir Physiol Neurobiol 132, 81-91 (2002).

40 Michelakis, E. D. et al. O2 sensing in the human ductus arteriosus: regulation of voltage-gated $\mathrm{K}+$ channels in smooth muscle cells by a mitochondrial redox sensor. Circulation research 91, 478-486 (2002).

41 Cogolludo, A. et al. Activation of neutral sphingomyelinase is involved in acute hypoxic pulmonary vasoconstriction. Cardiovasc Res 82, 296-302 (2009).

42 Archer, S. L. et al. Preferential expression and function of voltage-gated, O2sensitive $\mathrm{K}+$ channels in resistance pulmonary arteries explains regional heterogeneity in hypoxic pulmonary vasoconstriction: ionic diversity in smooth muscle cells. Circulation research 95, 308-318 (2004).

43 Yuan, X.-J., Goldman, W. F., Tod, M. L., Rubin, L. J. \& Blaustein, M. P. Hypoxia reduces potassium currents in cultured rat pulmonary but not mesenteric arterial myocytes. American Journal of Physiology-Lung Cellular and Molecular Physiology 264, L116-L123 (1993).

44 Young, S., Evans, K. \& Eu, J. Nitric oxide modulates branching morphogenesis in fetal rat lung explants. Am J Physiol Lung Cell Mol Physiol 282, L379-L385 (2002). 
45 Weigand, L., Foxson, J., Wang, J., Shimoda, L. A. \& Sylvester, J. T. Inhibition of hypoxic pulmonary vasoconstriction by antagonists of store-operated $\mathrm{Ca} 2+$ and nonselective cation channels. American journal of physiology 289, L5-L13 (2005).

46 Robertson, T. P., Aaronson, P. I. \& Ward, J. P. Hypoxic vasoconstriction and intracellular $\mathrm{Ca} 2+$ in pulmonary arteries: evidence for $\mathrm{PKC}$-independent $\mathrm{Ca} 2+$ sensitization. The American journal of physiology 268, H301-307 (1995).

47 Wang, J., Weigand, L., Foxson, J., Shimoda, L. A. \& Sylvester, J. T. Ca2+ signaling in hypoxic pulmonary vasoconstriction: effects of myosin light chain and Rho kinase antagonists. Am J Physiol Lung Cell Mol Physiol 293 (2007).

48 Nankervis, C. A. \& Miller, C. E. Developmental differences in response of mesenteric artery to acute hypoxia in vitro. Am J Physiol-Gastr L 274, G694G699 (1998).

49 Nowicki, P. T., Miller, C. E. \& Haun, S. E. Effects of arterial hypoxia and isoproterenol on in vitro postnatal intestinal circulation. Am J Physiol 255, H1144-1148 (1988).

50 Villamor, E., Ruiz, T., PerezVizcaino, F., Tamargo, J. \& Moro, M. Endotheliumderived nitric oxide-dependent response to hypoxia in piglet intrapulmonary arteries. Biol Neonate 72, $62-70$ (1997).

51 Nankervis, C. A., Giannone, P. J. \& Reber, K. M. The neonatal intestinal vasculature: contributing factors to necrotizing enterocolitis. Semin Perinato/32, 83-91 (2008).

52 Young, C. M., Kingma, S. D. \& Neu, J. Ischemia-reperfusion and neonatal intestinal injury. The Journal of pediatrics 158, e25-e28 (2011).

53 Wassink, G. et al. The ontogeny of hemodynamic responses to prolonged umbilical cord occlusion in fetal sheep. J Appl Physiol (1985) 103, 1311-1317 (2007).

54 Zhang, J. et al. Extracellular calcium-sensing receptor is critical in hypoxic pulmonary vasoconstriction. Antioxidants \& redox signaling 17, 471-484 (2012).

55 Olson, K. R. et al. Hydrogen sulfide as an oxygen sensor/transducer in vertebrate hypoxic vasoconstriction and hypoxic vasodilation. The Journal of experimental biology 209, 4011-4023 (2006). 
56 Gardner, D., Fletcher, A., Bloomfield, M., Fowden, A. \& Giussani, D. Effects of prevailing hypoxaemia, acidaemia or hypoglycaemia upon the cardiovascular, endocrine and metabolic responses to acute hypoxaemia in the ovine fetus. The Journal of physiology 540, 351-366 (2002).

57 Giussani, D. A. et al. Adrenergic and vasopressinergic contributions to the cardiovascular response to acute hypoxaemia in the llama fetus. The Journal of physiology 515, 233-241 (1999).

Acknowledgments: Supported, in part, by Spanish Ministerio de Ciencia e Innovación (grants SAF 2011-28150 and SAF 2014-55399-R). The funder had no role in study design, data collection and analysis, decision to publish, or preparation of the manuscript. 


\section{PART II}

THE L-ARGININE/NO PATHWAY AND THE RISK OF NEC

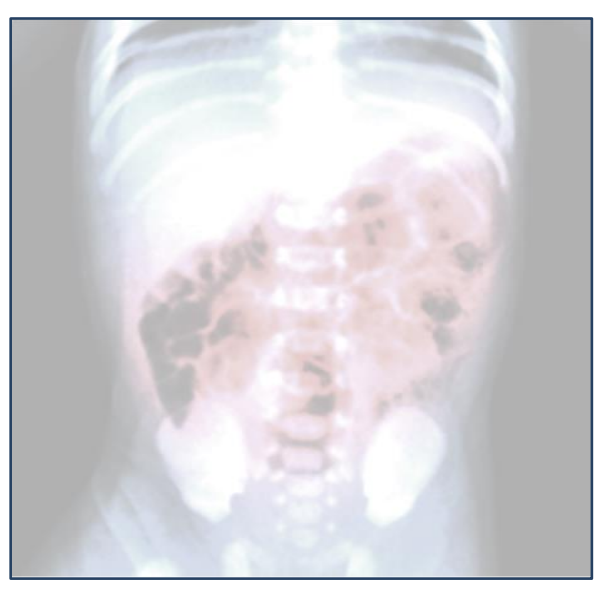


Chapter 5

CARBAMOYL PHOSPHATE SYNTHETASE POLYMORPHISMS AS A RISK FACTOR FOR NECROTIZING ENTEROCOLITIS

Rob M.J. Moonen, Aimee D.C. Paulussen, Nicole Y.P. Souren, Alfons G.H. Kessels,

M. Estela Rubio-Gozalbo and Eduardo Villamor

Pediatr Res 62, 188-190 (2007)

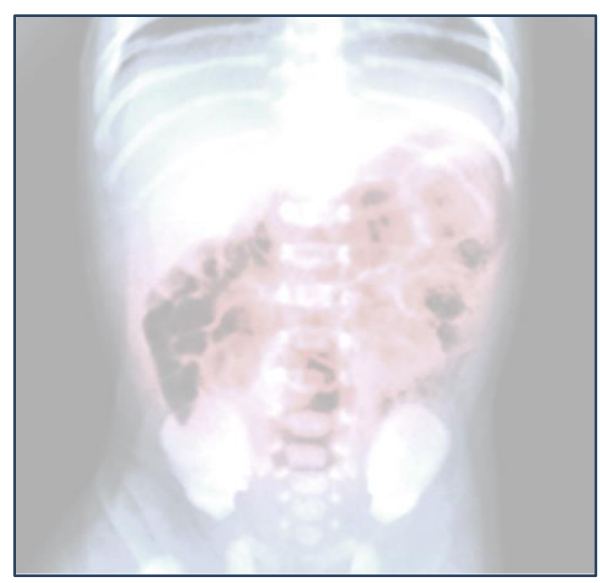




\section{ABSTRACT}

A C-to-A nucleotide transversion (T1405N) in the gene that encodes carbamoylphosphate synthetase 1 (CPS1) has been correlated with low plasma concentrations of $\mathrm{L}$-arginine in neonates. As plasma L-arginine concentrations are decreased in premature infants with necrotizing enterocolitis (NEC), we hypothesized that the CPS1 T1405N polymorphism would correlate with the presence of NEC. We analyzed the CPS1 genotypes for the T1405N polymorphism in 17 preterm infants ( $\leq 30 \mathrm{wk}$ and $<1500 \mathrm{~g}$ ) with established NEC, 34 preterm infants without NEC, and 25 healthy term infants. Distribution of genotypes did not differ between the NEC population (CC:AC:AA = 70.6\%:23.5\%:5.9\%) and the preterm control group (CC:AC:AA = 41.2\%:35.3\%:23.5\%; $P=0.110$ ) or the term group (CC:AC:AA $=44 \%: 48 \%: 8 \%$; $P=0.228$ ). The $C$ allele frequency was $82.4 \%$ in NEC and $58.8 \%$ in preterm control infants $(P=0.018)$ and analysis for linear trend demonstrated that incidence of NEC increased with the number of $C$ alleles $(P=0.037)$. The $C C$ genotype was associated with an increased risk of NEC in the preterm infants (odds ratio (OR) 3.43, 95\% confidence interval $(\mathrm{Cl}) 1.01-11.49, P=0.048)$, when compared with the grouped together AA/AC genotypes. These data suggest that the CPS1 T1405N polymorphism may be associated with the risk of NEC in preterm infants. 


\section{INTRODUCTION}

Necrotizing enterocolitis (NEC) remains a leading cause of morbidity and mortality in neonatal intensive care units. Although several predisposing factors have been identified, such as prematurity, enteral feeding, and infection, the pathogenesis of NEC remains elusive and accepted prevention and treatment strategies are lacking. ${ }^{1-8}$

Over the last several years, nitric oxide (NO) has received considerable attention in the pathophysiology of NEC as it pertains to the regulation of intestinal blood flow and plays a role in the maintenance of mucosal integrity and intestinal barrier function as well as in post-injury intestinal reparation. ${ }^{7,9} \mathrm{NO}$ is generated by NO synthase during the enzymatic conversion of L-arginine to L-citrulline. It has been suggested that the availability of L-arginine may be a factor limiting NO production, predisposing the immature gut to NEC. Several studies demonstrated that plasma arginine concentrations are decreased in premature infants with NEC. ${ }^{10-13}$ Moreover, arginine supplementation reduced the incidence of NEC. ${ }^{10}$ However, the cause of the relative arginine deficiency in premature infants with NEC remains unexplained.

Carbamoyl phosphate synthetase I (CPS1, OMIM 608307) is the rate-limiting enzyme catalyzing the first committed step of the urea cycle. ${ }^{14,15} \mathrm{~A}$ number of CPS1 polymorphisms have been found that appear to result in functional consequences affecting the downstream availability of the urea-cycle intermediates, including Larginine. ${ }^{14,15} \mathrm{~A}$ C-to-A nucleotide transversion at position 4332 of exon 36 of the CPS1 gene (chromosome 2q35) has been described, resulting in the substitution of asparagine for threonine at position $1405(\mathrm{T1405N})$, which is the critical $\mathrm{N}$ acetylglutamate-binding domain of the enzyme. ${ }^{14,15}$ Pearson et al. ${ }^{14}$ found that neonates (>35 wk of gestation) homozygous for the threonine 1405 variant of the CPS1 (CC genotype) had lower plasma L-arginine concentrations than neonates homozygous for the asparagine 1405 variant. In the present study, we hypothesized that the distribution of the polymorphism at position 1405 in the CPS1 gene would vary between preterm infants with and those without NEC. 


\section{METHODS}

\section{PATIENTS}

We performed a retrospective case control study based on the review of newborns infants with NEC hospitalized at the neonatology department of the University Hospital of Maastricht during the period January 1995-March 2006. Data on clinical characteristics were retrieved from a computerized database. From a population of 719 newborns with gestational age $\leq 30$ wk and birth weight $<1500$ grams, 36 patients with the diagnosis of established NEC (stage II and III according to the criteria proposed by Bell et al. ${ }^{1}$ and modified by Walsh and Kliegman ${ }^{8}$ ) were identified. Nine of the 36 patients had died and in 10 cases the patients were not located or the parents refused to let their children participate in the study. Two controls were matched to each of the 17 NEC cases on the basis of birth weight and gestational age. The incidence of other neonatal complications, such as infant respiratory distress syndrome, bronchopulmonary dysplasia, hypotension, sepsis, electrolyte disturbances, intraventricular hemorrhage/periventricular leukomalacia, convulsions, patent ductus arteriosus and retinopathy of prematurity as well as duration of mechanical ventilation, the duration of supplemental oxygen, and the administration of pre- and postnatal steroids and indomethacin were compared between the two preterm groups. A second control group of 25 healthy term infants was formed to examine the distribution of the CPS1 genotype in the local healthy population. All infants in this study were of Caucasian origin. The study was approved by an Institutional Ethical Committee (dossier MEC 04-140) and written informed consent from the parents was obtained.

\section{SAMPLES AND GENOTYPING}

Buccal cell samples for DNA testing were obtained with a sterile OmniSwab (Whatman) and collected in Eppendorf sterile PCR tubes. DNA was extracted using standard methods and stored at $-20^{\circ} \mathrm{C}$ until used for genotyping. A 214 bp fragment encompassing the $4332 \mathrm{C}>\mathrm{A}$ polymorphism in exon 36 of the CPS1 gene was amplified using PCR. Primers used were (forward) GCM357 5'TAAATGCAGCTGTTTGCCAC-3' and (reverse) GCM358 5'GACTTGCAATCAAGTAAGGTGAAA-3'. The PCR mix consisted of 1x GeneAmp PCR Buffer II (Perkin Elmer), 0.2 mM dNTP's (Pharmacia), 1.5 mM MgCl 2 (Perkin Elmer), 
$250 \mathrm{nM}$ of both primers and $0.025 \mathrm{U} / \mu \mathrm{l}$ of AmpliTaq Gold (Perkin Elmer). Thermocycling conditions started with an initial denaturation of $10 \mathrm{~min} 95^{\circ} \mathrm{C}$, followed by 35 cycles of $95^{\circ} \mathrm{C}(45 \mathrm{sec}), 55^{\circ} \mathrm{C}(45 \mathrm{sec}), 72^{\circ} \mathrm{C}(45 \mathrm{sec})$ and ended with a final extension step of $10 \mathrm{~min} 72^{\circ} \mathrm{C}$. The PCR product was purified and directly sequenced using the reverse primer.

\section{STATISTICAL ANALYSIS}

Discrete variables are expressed as counts or percentages, and differences were compared using the chi-square test. Results for continuous variables are expressed as mean (SD) or, if variables were not normally distributed, as median (interquartile range) and compared with the $t$-test or the Mann-Whitney $U$ test, as appropriate.

Differences in allelic frequencies and genotype distributions between the investigated populations, as well as the presence of Hardy-Weinberg equilibrium, were tested by the chi-square test. Chi-square analysis was performed for linear trend conformity, according to the presence of zero, one, or two $C$ alleles. Odds ratio (OR) and $95 \%$ confidence intervals $(\mathrm{Cl})$ were calculated as a measure of association between genotype and the presence of NEC in preterm infants. To be able to correct for known risk factors for NEC (gestational age and birth weight) binary logistic regression analysis was performed. A $P$ value of $<0.05$ was considered statistically significant. 


\section{RESULTS}

\section{PATIENT CHARACTERISTICS}

Seventeen preterm infants with NEC (Bell stage II or greater) and 34 preterm infants without NEC were enrolled in the study. There were no significant differences in baseline characteristics and neonatal complications between the two preterm populations (Table 1). In addition, there was no significant difference in administration of prenatal or postnatal steroids or indomethacin. Stage II NEC was present in 9 infants and stage III in 8 infants. Suspected NEC was present in 3 infants of the preterm control group. A second control group consisted of 25 healthy term infants (12 males) with a mean birth weight of $3330 \mathrm{~g}$ (SD 464) and a mean gestational age of $39.4 \mathrm{wk}$ (SD 1.5).

\section{ANALYSIS OF GENOTYPES AND ALLELIC FREQUENCIES}

Genotype frequencies according to the study population are summarized in Table 2. The distribution of the CPS1 genotypes for the polymorphism at position 1405 in each studied population fulfilled Hardy-Weinberg criteria. Genotype distributions (CC denotes homozygosity for the C-encoded Thr1405 variant, AA homozygosity for the Aencoded Asn1405 variant, and AC heterozygosity for this polymorphism at position 1405 ) of the term control population (CC:AC: $A A=44 \%: 48 \%: 8 \%$ ) did not differ $(P=0.836)$ from those reported by Summar et al. ${ }^{15}$ in 460 healthy adults in the U.S.A (CC:AC:AA $=42 \%: 46 \%: 12 \%$ ). The preterm control population showed a higher proportion of the AA genotype (CC:AC:AA $=41.2 \%: 35.3 \%: 21.5 \%$ ) but comparison with the term control population did not reach statistical significance $(P=0.116)$.

The distribution of genotypes in the NEC population (CC:AC:AA = 70.6\%:23.5\%:5.9\%) was not significantly different when compared with the term control group $(P=0.228)$ or the preterm control group ( $P=0.110$ ). The frequency of the $C$ allele was $82,4 \%$ in NEC infants and $58.8 \%$ in the preterm control group $(P=0,018)$, and the analysis for a linear trend regarding the presence of zero, one or two $C$ alleles demonstrated that the incidence of NEC increased with the number of $C$ alleles $(P=0.037)$. In addition, preterm infants with the $\mathrm{CC}$ genotype presented a higher risk of NEC (unadjusted OR 
$=3.43,95 \% \mathrm{Cl}: 1.01-11.49, P=0.048)$ than grouped together homozygous and heterozygous carriers of the A-encoded allele (AA / AC genotype).

Table 1. Baseline characteristics and neonatal complications in the preterm populations

\begin{tabular}{lccc}
\hline & $\begin{array}{c}\text { NEC group } \\
(\mathbf{n}=\mathbf{1 7})\end{array}$ & $\begin{array}{c}\text { Preterm control } \\
\text { group (n = 34) }\end{array}$ & P value \\
\hline Birth weight (g) & $912($ SD 169) & 978 (SD 297) & 0.315 \\
Gestational age (wks) & $28.4[26.9-29.4]$ & $28.6[27.0-29.2]$ & 0.976 \\
Male sex & $8(47)$ & $22(65)$ & 0.227 \\
Apgar score & & & \\
\multicolumn{1}{c}{ after 1 minute } & $5[4-9]$ & $5[3-8]$ & 0.470 \\
after 5 minutes & $9[7-9]$ & $8[6-9]$ & 0.751 \\
Supplemental oxygen (days) & $9[1-12]$ & $11.5[1-44]$ & 0.303 \\
Mechanical ventilation (days) & $10[3-19]$ & $4[0-17]$ & 0.497 \\
IRDS & $10(59)$ & $27(79)$ & 0.120 \\
BPD & $5(29)$ & $12(35)$ & 0.674 \\
Hypotension & $8(47)$ & $22(65)$ & 0.227 \\
Sepsis & $15(88)$ & $24(71)$ & 0.161 \\
Electrolyte disturbances & $13(76)$ & $25(74)$ & 0.820 \\
IVH/PVL & $7(41)$ & $12(35)$ & 0.682 \\
Convulsions & $5(29)$ & $4(12)$ & 0.119 \\
PDA & $2(12)$ & $7(21)$ & 0.436 \\
ROP & $7(41)$ & $6(18)$ & 0.069 \\
\hline
\end{tabular}

Results are expressed as mean (SD), median [interquartile range], or absolute numbers of patients (percentage). IRDS, infant respiratory distress syndrome; BPD, bronchopulmonary dys-plasia; IVH/PVL, intraventricular hemorrhage/periventricular leukomalacia; PDA, patent ductus arteriosus; ROP, retinopathy of prematurity. 
Table 2. distribution of the CPS1 genotypes for the polymorphism at position 1405

\begin{tabular}{|c|c|c|c|c|}
\hline Population & No. of infants & CC & $A C$ & AA \\
\hline Summar et al. ${ }^{15}$ & - & - (42) & $-(46)$ & - (12) \\
\hline Term control group & 25 & $11(44.0)$ & $12(48.0)$ & $2(8.0)$ \\
\hline Preterm control group & 34 & $14(41.2)$ & $12(35.3)$ & $8(23.5)$ \\
\hline NEC group & 17 & $12(70.6)$ & $4(23.5)$ & $1(5.9)$ \\
\hline
\end{tabular}

Results are expressed as absolute numbers of patients (percentage). CC denotes homozygosity for the C-encoded Thr1405 variant, AA homozygosity for the A-encoded Asn 1405 variant, and $A C$ heterozygosity for this polymorphism. OR = 3.43 (95\% Cl: 1.01 11.49) for preterm with NEC versus preterm without NEC (CC vs. $A C+A A)$.

In the logistic regression model, neither gestational age $(P=0.952)$ nor birth weight $(P=0.650)$ were significantly associated with NEC. However, when adjusted for birth weight and gestational age the association between CC genotype and NEC did not remain significant (adjusted OR $=3.246,95 \% \mathrm{Cl}: 0.91-11.52, P=0.069$ ).

Although the CC genotype was present in 7 of the 8 infants with stage III NEC and in 5 of the 9 infants with stage II NEC, there was not a significant increased risk for stage III NEC versus stage II NEC in the carriers of the CC genotype (OR $=0.18,95 \% \mathrm{Cl}$ : $0.02-1.71, P=0.149)$ and linear trend analysis demonstrated that the presence of the $\mathrm{C}$-allele was not significantly increased in the stage III NEC patients $(P=0.496)$. None of the infants with stage II NEC and only 1 infant with stage III NEC presented the AA genotype.

No significant difference in the prevalence of the CC genotype was found when comparing the NEC population with the term control population $(P=0.089$, analysis for linear trend $P=0.145$ ) or when comparing the preterm control population with the term control population $(P=0.828$, analysis for linear trend $P=0.338)$. 


\section{DISCUSSION}

Identifying premature infants at increased risk for developing NEC remains an important but elusive objective. Prematurity is the only factor consistently found in epidemiological studies to be an independent determinant of NEC. ${ }^{3,4,6,9}$ However, NEC affects only a minority of premature infants, which suggest an individual susceptibility toward the disease. Genetic polymorphisms might be an important factor in this individual susceptibility. ${ }^{16}$

Using case-control methodology we examined the relationship between a single nucleotide polymorphism (C-to-A nucleotide transversion in exon 36) of the gene that encodes CPS1, the rate-limiting enzyme in the urea cycle production of the NO precursor L-arginine, and the presence of NEC in preterm infants. We found that patients with NEC showed an overrepresentation of the C-encoded variant of CPS1. Linear trend analysis demonstrated the quantitative increase of $C$ allele in NEC and homozygosity for $\mathrm{C}$ allele increased the risk of having NEC. Although the case and the controls were well matched and there was no significant association of gestational age or birth weight with NEC in the logistic regression model, when adjusted for these two known risk factors for NEC, the OR did not remain significant. Therefore, whether the CC genotype is an independent risk factor for NEC needs to be confirmed in studies involving larger populations.

Reduced serum concentrations of $\mathrm{L}$-arginine have been reported in term neonates with the CC genotype ${ }^{14}$ and in preterm infants with NEC. ${ }^{10-12}$ Although the explanation may lie in other enzymes or transporters in the urea cycle and NO pathway, our findings might represent a connection point between these previous findings and suggest a potential genetic vulnerability to NEC.

Endogenous synthesis of arginine is crucial for maintaining arginine homeostasis in neonates. ${ }^{13}$ Further, both metabolic and molecular studies indicate that the underdevelopment of intestinal arginine synthesis may be primarily responsible for hypoargininemia in preterm neonates. ${ }^{13}$ In premature infants, in whom the urea cycle is not fully developed ${ }^{13,14}$, further decrease in arginine and citrulline production due to 
genetically determined variations in CPS1 function could affect the production of NO and the subsequent development of NEC. In this sense, the low prevalence of AA homozygosity among the infants with NEC is noteworthy. Similarly, Pearson et al. ${ }^{14}$ have reported a lack of AA homozygosity among neonates with pulmonary hypertension and Summar et al. ${ }^{17}$ reported a reduced rate of post-cardiac surgeryrelated pulmonary hypertension and hepatic veno-occlusive disease after bone marrow transplantation in patients with the AA genotype. They speculate that individuals with the AA genotype may have an advantage in terms of urea-cycle function and interrelated metabolic processes, especially under conditions of environmental stress.

NEC is a complex multifactorial disease, and an isolated genetic derangement may not be sufficient to account for the entire spectrum of its pathophysiology. ${ }^{9}$ Previous studies have associated NEC with genetic polymorphisms of interleukin (IL)-4-receptor $\alpha$ chain and IL-18. ${ }^{16,18,19}$ Our findings provide the preliminary evidence that a single nucleotide polymorphism in the CPS1 gene is associated with the risk of NEC. However, our study is limited by its retrospective nature, the relatively small size of the sample, its use of data from a single tertiary care institution, the lack of data of patients who died from NEC, and the lack of confirmation of the relationship between CPS1 polymorphisms and levels of L-arginine in preterm infants. Therefore, our data should be confirmed in larger populations, and the relationships among CPS1 polymorphisms, L-arginine, and the incidence of NEC warrant further investigation in a prospective cohort study. 


\section{REFERENCES}

1 Bell, E. F. Preventing necrotizing enterocolitis: what works and how safe? Pediatrics 115, 173-174 (2005).

2 Bell, M. J. et al. Neonatal necrotizing enterocolitis. Therapeutic decisions based upon clinical staging. Annals of surgery 187, 1-7 (1978).

3 Caplan, M. S. \& Jilling, T. New concepts in necrotizing enterocolitis. Current opinion in pediatrics 13, 111-115 (2001).

4 Lee, J. S. \& Polin, R. A. Treatment and prevention of necrotizing enterocolitis. Seminars in neonatology : SN 8, 449-459 (2003).

5 Martin, C. R. \& Walker, W. A. Intestinal immune defences and the inflammatory response in necrotising enterocolitis. Seminars in fetal \& neonatal medicine 11, 369-377 (2006).

6 Reber, K. M. \& Nankervis, C. A. Necrotizing enterocolitis: preventative strategies. Clinics in perinatology 31, 157-167 (2004).

7 Reber, K. M., Nankervis, C. A. \& Nowicki, P. T. Newborn intestinal circulation. Physiology and pathophysiology. Clinics in perinatology 29, 23-39 (2002).

8 Walsh, M. C. \& Kliegman, R. M. Necrotizing enterocolitis: treatment based on staging criteria. Pediatric clinics of North America 33, 179-201 (1986).

9 Upperman, J. S. et al. Mechanisms of nitric oxide-mediated intestinal barrier failure in necrotizing enterocolitis. Seminars in pediatric surgery 14, 159-166 (2005).

10 Amin, H. J. et al. Arginine supplementation prevents necrotizing enterocolitis in the premature infant. J Pediatr 140, 425-431 (2002).

11 Becker, R. M. et al. Reduced serum amino acid concentrations in infants with necrotizing enterocolitis. J Pediatr 137, 785-793 (2000).

12 Zamora, S. A. et al. Plasma L-arginine concentrations in premature infants with necrotizing enterocolitis. J Pediatr 131, 226-232 (1997).

13 Wu, G., Jaeger, L. A., Bazer, F. W. \& Rhoads, J. M. Arginine deficiency in preterm infants: biochemical mechanisms and nutritional implications. The Journal of nutritional biochemistry 15, 442-451 (2004). 
14 Pearson, D. L. et al. Neonatal pulmonary hypertension--urea-cycle intermediates, nitric oxide production, and carbamoyl-phosphate synthetase function. The New England journal of medicine 344, 1832-1838 (2001).

15 Summar, M. L. et al. Environmentally determined genetic expression: clinical correlates with molecular variants of carbamyl phosphate synthetase I. Molecular genetics and metabolism 81 Suppl 1, S12-19 (2004).

16 Treszl, A., Tulassay, T. \& Vasarhelyi, B. Genetic basis for necrotizing enterocolitis--risk factors and their relations to genetic polymorphisms. Frontiers in bioscience : a journal and virtual library 11, 570-580 (2006).

17 Summar, M. L. et al. Relationship between carbamoyl-phosphate synthetase genotype and systemic vascular function. Hypertension 43, 186-191 (2004).

18 Heninger, E. et al. Genetic variants of the interleukin-18 promoter region (-607) influence the course of necrotising enterocolitis in very low birth weight neonates. European journal of pediatrics 161, 410-411 (2002).

19 Treszl, A. et al. Lower prevalence of IL-4 receptor alpha-chain gene $G$ variant in very-low-birth-weight infants with necrotizing enterocolitis. Journal of pediatric surgery 38, 1374-1378 (2003). 
Chapter 6

ASSOCIATION BETWEEN THE P.THR1406ASN POLYMORPHISM OF THE CARBAMOYL-PHOSPHATE SYNTHASE 1 GENE AND NECROTIZING ENTEROCOLITIS: A PROSPECTIVE MULTICENTER STUDY

Rob M.J. Moonen, Giacomo Cavallaro, Maurice J. Huizing, Gema E. González-Luis, Fabio Mosca and Eduardo Villamor Accepted for publication Sci Rep (20th October 2016)

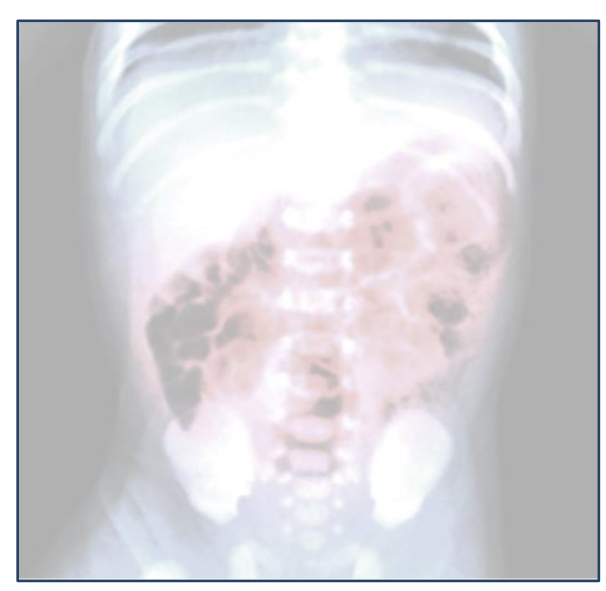




\section{ABSTRACT}

The p.Thr1406Asn (rs1047891) polymorphism of the carbamoyl-phosphate synthetase 1 (CPS1) gene has been linked to functional consequences affecting the downstream availability of the nitric oxide precursor L-arginine. L-arginine concentrations are decreased in preterm infants with necrotizing enterocolitis (NEC). In this multicenter prospective study, we investigated the association of the p.Thr1406Asn polymorphism with NEC in 477 preterm infants ( 36 cases of NEC) from 4 European neonatal intensive care units (Maastricht, Las Palmas de Gran Canaria, Mantova, and Milan). Allele and genotype frequencies of the p.Thr1406Asn polymorphism did not significantly differ between the infants with and without NEC. In contrast, the minor A-allele was significantly less frequent in the group of 64 infants with the combined outcome NEC or death before 34 weeks of corrected gestational age than in the infants without the outcome ( 0.20 vs. $0.31, P=0.03$ ). In addition, a significant negative association of the A-allele with the combined outcome NEC or death was found using the dominant (adjusted odds ratio, aOR: $0.54,95 \% \mathrm{Cl} 0.29-0.99$ ) and the additive (aOR $0.58,95 \%$ $\mathrm{Cl} 0.36-0.93)$ genetic models. In conclusion, our study provides further evidence that a functional variant of the CPS1 gene may contribute to NEC susceptibility. 


\section{INTRODUCTION}

Necrotizing enterocolitis (NEC) remains a significant cause of morbidity and mortality in neonatal intensive care units. Although several predisposing factors have been identified, the exact etiology of NEC is yet elusive. The combination of genetic predisposition, immaturity of gastrointestinal motility, digestive ability, intestinal barrier function, immune defense and microcirculatory regulation accompanied by a strong likelihood of abnormal microbial colonization in the intestine, leads to a confluence of predisposing factors. ${ }^{1-9}$

Nitric oxide (NO) has received increasing attention in the pathophysiology of NEC, as it participates in the regulation of intestinal blood flow and plays a key role in the maintenance of mucosal integrity, intestinal barrier function, and post-injury intestinal repair. ${ }^{10-12} \mathrm{NO}$ is generated by NO synthase (NOS) during the enzymatic conversion of L-arginine to L-citrulline. The NOS substrate L-arginine is an essential amino acid for young mammals. ${ }^{13,14}$ Metabolic and molecular studies indicate that the underdevelopment of intestinal arginine synthesis may be primarily responsible for remarkably low plasma arginine concentrations in preterm neonates. ${ }^{14,15}$ Several studies demonstrated that plasma arginine concentrations are even more decreased in preterm infants with NEC. ${ }^{14,16-19}$ Moreover, data from two small randomized controlled studies ${ }^{16,20}$, pooled in a meta-analysis ${ }^{21}$, suggest that arginine supplementation reduces the incidence of NEC in preterm infants.

Arginine is a urea cycle intermediate. The first step in the urea cycle occurs inside the mitochondrion and is catalyzed by the enzyme, carbamoyl-phosphate synthetase I (CPS1, EC 6.3.4.16). ${ }^{22}$ CPS1 deficiency in humans is a rare autosomal recessive inborn error of the urea cycle leading to hyperammonemia. Deficiency can be primary, due to mutations in the CPS1 gene (OMIM 608307, HGNC:2323) or secondary, due to the lack of the essential cofactor $\mathrm{N}$-acetyl-L-glutamate. ${ }^{22}$ In addition, a number of functional single nucleotide polymorphisms (SNPs) have been identified in the CPS1 gene. ${ }^{22-24}$ One of those SNPs (p.Thr1406Asn also published as T1405N; rs1047891 formerly designated as rs7422339) has been linked to functional consequences affecting the downstream availability of urea-cycle intermediates, including L- 
arginine. ${ }^{22-24}$ The SNP p.Thr1406Asn is a C-to-A nucleotide transversion (c.4217C >A) in exon 36, which results in the substitution of asparagine (Asn) for threonine (Thr) in the critical $\mathrm{N}$-acetylglutamate-binding domain. ${ }^{22,25}$ The C-encoded Thr form of the polymorphism is considered the evolutionarily conserved version and the less frequent, A-encoded, Asn variant appears to be a relatively new, gain-of-function mutation. ${ }^{15,25}$ It has been suggested that the A-allele may confer an advantage in terms of NO production, especially under conditions of environmental stress. ${ }^{15}$ Previous studies demonstrated the association of the CPS1 p.Thr1406Asn genotype with clinical situations where endogenous NO production is critically important, such as persistent pulmonary hypertension of the newborn ${ }^{15}$, pulmonary hypertension following surgical repair of congenital heart defects ${ }^{26}$, and hepatic veno-occlusive disease after bone marrow transplantation ${ }^{23}$.

Several years ago, we reported in a retrospective series of 17 preterm infants with NEC and 34 controls that patients with NEC showed an underrepresentation of the Aencoded variant of the p.Thr1406Asn polymorphism of CPS1. ${ }^{27}$ Those results suggested that the A-allele conferred protection against NEC and warranted confirmation using a prospective design and larger sample size. Herein we report the results of such study, involving 477 preterm infants (36 cases of NEC) from four neonatal intensive care units located in three different European countries (Spain, Italy, and the Netherlands). Since death in the first weeks of life is a competing outcome for NEC, we also analyzed the association of the p.Thr1406Asn polymorphism with the combined outcome NEC or death before 34 weeks of corrected gestational age (GA). 


\section{METHODS}

\section{PATIENTS}

The study was approved by the Institutional Review Boards (IRBs) of the Maastricht University Medical Center (the Netherlands, registration number MEC 04-140), Hospital Universitario Materno-Infantil de Canarias (Las Palmas de Gran Canaria, Spain), Carlo Poma Hospital (Mantova, Italy) and Ospedale Maggiore Policlinico (Milan, Italy). The study was conducted according to institutional and IRB guidelines and regulations and registered in ClinicalTrials.gov Protocol Registration System (NCT00554866, ID 07-2-018, November 6, 2007). The manuscript was drafted according to the STROBE statement (http://www.strobestatement.org/). All infants with a GA $\leq 30$ weeks and birth weight (BW) $\leq 1500 \mathrm{~g}$ born between $1^{\text {st }}$ July 2007 and $30^{\text {th }}$ June 2012 and admitted to the level III neonatal intensive care unit of the above mentioned centers (Carlo Poma Hospital: inclusion until 31 ${ }^{\text {st }}$ December 2010; Ospedale Maggiore Policlinico: inclusion from $1^{\text {st }}$ March 2011) were eligible for participation in the study. Written informed consent from the parents was obtained. As reported in a previous study, buccal cell samples were obtained from 96 healthy term infants (25 in Maastricht, 31 in Las Palmas de Gran Canaria and 40 in Mantova). ${ }^{30}$

\section{DEFINITION OF CLINICAL CHARACTERISTICS AND OUTCOMES}

Data on clinical characteristics and outcomes were obtained from the medical records. GA was determined by the last menstrual period and early ultrasounds (before 20 weeks of gestation). Small for GA was defined as BW for GA below the sex-specific $10^{\text {th }}$ percentile. Chorioamnionitis was defined as every clinical suspicion of infection of the chorion, amnion, amniotic fluid, placenta, or a combination as judged by the obstetrician. Prolonged rupture of membranes was defined as rupture of membranes $>24$ hours before delivery. Prenatal exposure to a single course of antenatal steroids was defined as two doses of betamethasone administered $24 \mathrm{~h}$ apart and exposure to a partial course of antenatal steroids was defined as administration of a single dose of betamethasone $<24 \mathrm{~h}$ prior to delivery.

NEC was defined as Bell stage II disease or greater. At the conclusion of the study, all cases of NEC were reviewed in a blinded fashion by a panel of 4 investigators of the 
study. Cases of spontaneous intestinal perforation (i.e., without pathologic evidence of $\mathrm{NEC}$ ) were excluded from the investigation. Since it was considered that some infants who died in the first weeks may have developed NEC if they had survived, we also analyzed the composite outcome of NEC or death before 34 weeks of corrected GA.

Respiratory distress syndrome (RDS) was defined as requirement for oxygen supplementation or respiratory support due to tachypnea, grunting, nasal flaring, retractions, or cyanosis. Bronchopulmonary dysplasia (BPD) was defined as a supplemental oxygen requirement at 36 weeks of corrected GA to maintain oxygen saturation $>90 \% .{ }^{31}$ Arterial hypotension was defined as the need for volume expansion or inotropic support. Patent ductus arteriosus (PDA) was defined as a requirement for indomethacin or ibuprofen and/or surgical ligation. A diagnosis of sepsis required signs of generalized infection, a positive blood culture, and antibiotic therapy. Intraventricular hemorrhage (IVH) was classified by using the 4-level grading system..$^{32}$ Grade $<2$ IVHs were not included in the analysis. Retinopathy of prematurity (ROP) was defined as stage II or higher.

\section{SAMPLES AND GENOTYPING}

Buccal cell samples for DNA testing were obtained with a sterile OmniSwab (Whatman), and collected in Eppendorf sterile PCR tubes and stored at $-80{ }^{\circ} \mathrm{C}$ until further analysis. The samples obtained in Spain and Italy were transported on dry ice to Maastricht where all the analyses were performed. DNA was extracted using standard methods and stored at $-20^{\circ} \mathrm{C}$ until genotyping. A 214-bp fragment encompassing the p.Thr1406Asn polymorphism in exon 36 of the CPS1 gene was amplified using polymerase chain reaction (PCR). Primers used were (forward) GCM357 5'-TAAATGCAGCTGTTTGCCAC-3' and (reverse) GCM358 5'GACTTGCAATCAAGTAAGGTGAAA-3'. The PCR mix consisted of 1X GeneAmp PCR Buffer II (Perkin-Elmer, Branchburg, NJ), $0.2 \mathrm{mM}$ deoxyribonucleoside triphosphate (Pharmacia Biotech, Bridgewater, NJ), $1.5 \mathrm{mM} \mathrm{MgCl} 2$ (Perkin-Elmer, Branchburg, NJ), $250 \mathrm{nM}$ of both primers, and $0.025 \mathrm{U} / \mu \mathrm{L}$ of AmpliTaq Gold (PerkinElmer, Branchburg, NJ). Thermocycling conditions started with an initial denaturation of $10 \mathrm{~min} 95^{\circ} \mathrm{C}$, followed by 35 cycles of $95^{\circ} \mathrm{C}(45 \mathrm{~s}), 55^{\circ} \mathrm{C}(45 \mathrm{~s}), 72^{\circ} \mathrm{C}(45 \mathrm{~s})$, and 
ended with a final extension step of $10 \mathrm{~min}$ at $72^{\circ} \mathrm{C}$. The PCR product was purified and directly sequenced using the reverse primer.

\section{STATISTICAL ANALYSIS}

Sample size was calculated based on data from our previous study. ${ }^{27}$ Given an expected population incidence of NEC of $5 \%$, expected frequencies for $\mathrm{CC}$ homozygosity of 0.7 (NEC group) and 0.4 (control group), alpha level $=0.05$, and power of $0.8,440$ infants were needed to detect an odds ratio (OR) significantly different from 1.

Categorical variables were expressed as counts or percentages and compared using the chi-square test. Continuous variables were expressed as mean (SD) if they followed a normal distribution and compared using unpaired, two-sided $t$-test. If not normally distributed, continuous variables were expressed as median values (interquartile range, IQR; 25th-75th percentile) and compared using the MannWhitney $U$ test. The Kolmogorov-Smirnov test was used to test for normal distribution of continuous data.

Differences in allelic frequencies and genotype distributions between the investigated populations, as well as Hardy-Weinberg equilibrium (HWE) for genotype distribution were assessed using a chi-square test. The Hardy-Weinberg law states that $q^{2}+2 p q$ $+p^{2}=1$, where $p$ and $q$ are allele frequencies in a two-allele system. Logistic regression analysis was used to compute the ORs and their 95\% confidence intervals (Cl) for NEC and the combined outcome NEC or death before 34 weeks of corrected GA based on genotype after accounting for the covariates which were significantly different between the groups and are known risk factors for developing NEC. Different genetic models were used to analyze the effect of the risk allele, including the general allelic (multiplicative or codominant model), dominant, recessive and additive models. Assuming a genetic penetrance parameter $\gamma(\gamma>1)$, a multiplicative model indicates that the risk of disease is increased $y$-fold with each additional copy of the risk allele; an additive model indicates that risk of disease is increased $\gamma$-fold for the genotype with one copy of the risk allele and $2 \mathrm{y}$-fold for the genotype with two copies of the risk allele; a common recessive model indicates that two copies of the risk allele are 
required for a $y$-fold increase in disease risk, and a common dominant model indicates that either one or two copies of the risk allele are required for a $\mathrm{y}$-fold increase in disease risk. ${ }^{33}$ The major allele was considered as a reference and the interactions were tested in the different models by multivariable logistic regression model. All the statistical analyses were performed using IBM SPSS Statistics for Windows, Version 22.0. (IBM Corporation, Armonk, NY, USA) and conducted at the $P<0.05$ level of significance. 


\section{RESULTS}

\section{PATIENT CHARACTERISTICS}

From 615 eligible infants, 477 (36 with NEC Bell stage II or greater) were included in the study (Fig. 1). Stage II NEC was present in 23 infants and stage III in 13 infants. In 21 cases surgery was required. The median age at the onset of NEC was 20 days (range 4-87, IQR 12-31). Nine of the cases of NEC occurred in Maastricht (5 stage II NEC, 4 stage III NEC), 16 in Las Palmas (9 stage II NEC, 7 stage III NEC), and 11 in Italy (9 stage II NEC, 2 stage III NEC). Single intestinal perforation was present in 5 infants (1 in Maastricht, 4 in Italy). Demographic and clinical characteristics of the infants with and without NEC are shown and compared in Table 1. Mean GA, mean BW and median Apgar score at 1 min of infants with NEC were significantly lower than in infants without NEC. In addition, infants with NEC showed a higher incidence of vaginal delivery, mechanical ventilation, BPD, hypotension, IVH, PVL, PDA, ROP and mortality. We adjusted for GA, BW, Apgar score at $1 \mathrm{~min}$, mechanical ventilation, hypotension, and PDA in the subsequent logistic regression analysis.

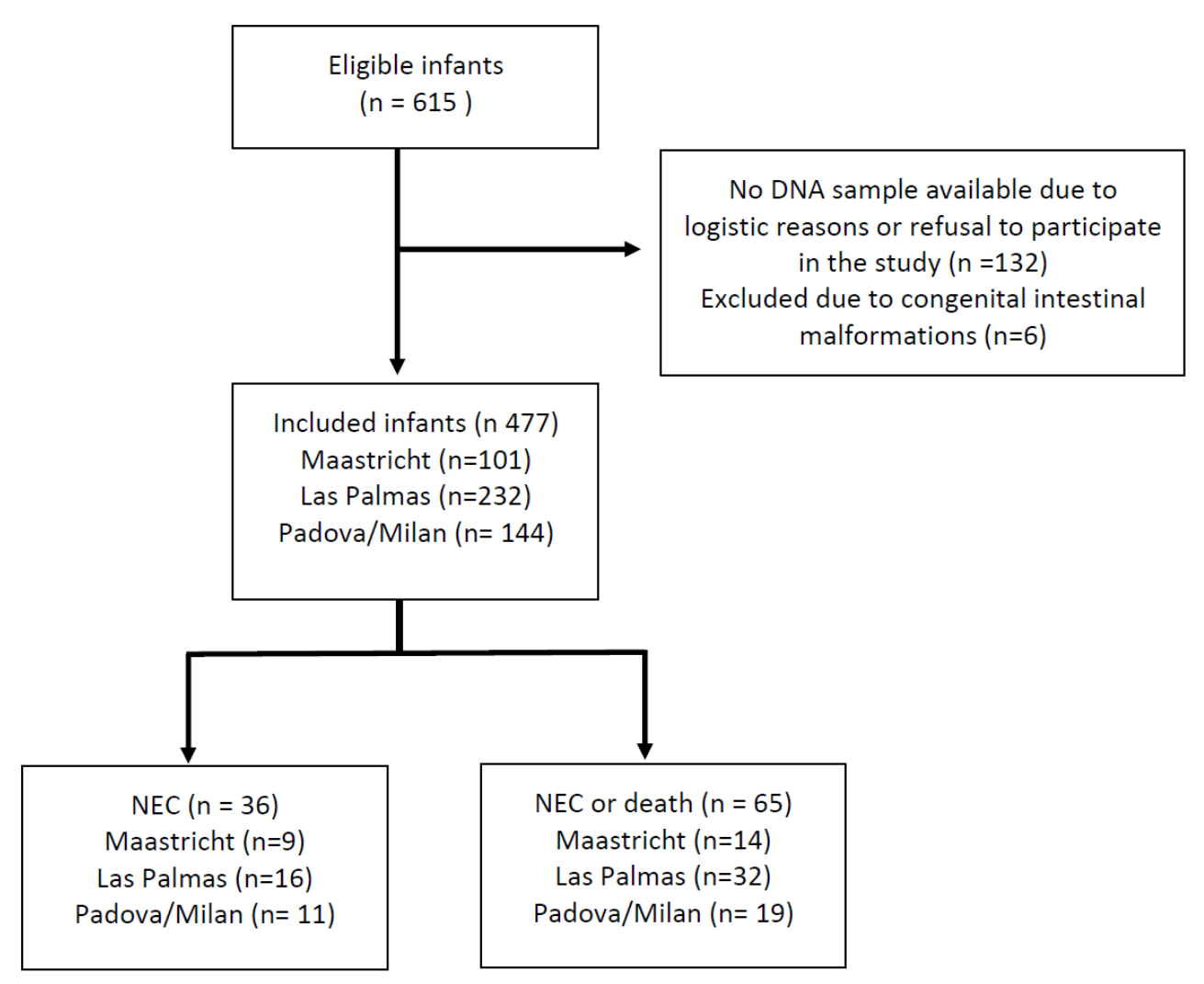

Figure 1. Flowchart of study participants. 
Table 1. Baseline characteristics and neonatal complications in preterm infants with and without NEC.

\begin{tabular}{|c|c|c|c|c|c|}
\hline & $\begin{array}{c}\text { NEC -yes } \\
(n=36)\end{array}$ & $\begin{array}{c}n \text { data } \\
\text { missing }\end{array}$ & $\begin{array}{l}\text { NEC-no } \\
(n=441)\end{array}$ & $\begin{array}{c}n \text { data } \\
\text { missing }\end{array}$ & $P$ value \\
\hline Birth weight (g) & 844 (SD 216) & 0 & 1016 (SD 266) & 0 & .000 \\
\hline Gestational age (wks) & 26.7 (SD 1.9) & 0 & 27.9 (SD 1.9) & 0 & .000 \\
\hline Male sex & $17(47.2)$ & 0 & $238(54.0)$ & 0 & .419 \\
\hline Prenatal steroids & $28(80.0)$ & 1 & $375(88.2)$ & 16 & .361 \\
\hline Preecclampsia & $3(8.6)$ & 1 & $68(15.6)$ & 5 & .264 \\
\hline Chorioamnionitis & $3(8.3)$ & 0 & $58(13.3)$ & 5 & .393 \\
\hline PROM & $6(16.7)$ & 0 & $118(27.2)$ & 7 & .169 \\
\hline Vaginal delivery & $17(58.6)$ & 0 & $147(43.2)$ & 2 & .042 \\
\hline Apgar (1 min) & 5 [3-7] & 1 & $6[4-8]$ & 5 & .026 \\
\hline Apgar (5 min) & 8 [6-9] & 1 & 8 [7-9] & 6 & .114 \\
\hline RDS & $30(83.3)$ & 0 & $378(85.9)$ & 1 & .671 \\
\hline Mechanical vent. & $32(88.9)$ & 0 & $279(64.0)$ & 5 & .002 \\
\hline BPD & $21(58.3)$ & 0 & $137(31.3)$ & 3 & .001 \\
\hline Hypotension & $26(72.2)$ & 0 & $175(40.1)$ & 5 & .000 \\
\hline Sepsis & $23(67.6)$ & 2 & $227(51.7)$ & 2 & .073 \\
\hline $\mathrm{IVH}$ & $16(44.4)$ & 0 & $110(25.1)$ & 3 & .012 \\
\hline PVL & $4(11.1)$ & 0 & $14(3.2)$ & 4 & .017 \\
\hline PDA & $25(71.4)$ & 1 & $233(53.2)$ & 3 & .037 \\
\hline ROP & $13(41.9)$ & 5 & $85(20.5)$ & 27 & .006 \\
\hline Mortality & 8 (22.2) & 0 & $42(9.5)$ & 0 & .017 \\
\hline Death before 34 wks & $5(13,9)$ & 0 & $29(6.6)$ & 0 & .101 \\
\hline
\end{tabular}

Results are expressed as mean (SD), median [interquartile range] or absolute numbers of patients (percentage). NEC: necrotizing enterocolitis ( $\geq$ stage II); PROM: prolonged rupture of membranes; RDS: respiratory distress syndrome; BPD: bronchopulmonary dysplasia; IVH: intraventricular hemorrhage ( $\geq$ grade 2); PVL: periventricular leukomalacia; PDA: patent ductus arteriosus; ROP: retinopathy of prematurity ( $\geq$ stage II). 
The demographic and clinical characteristics of the infants with and without the combined outcome NEC or death before 34 weeks of corrected GA are shown and compared in Table 2. Mean GA, mean BW and median Apgar score at 1 and 5 min of infants with NEC or death were significantly lower than in infants without the combined outcome. In addition, infants with the combined outcome NEC or death showed a higher incidence of vaginal delivery, mechanical ventilation, hypotension, IVH and PDA. We adjusted for GA, BW, Apgar score after at 1 and $5 \mathrm{~min}$, mechanical ventilation, hypotension and PDA in the subsequent logistic regression analysis. 
Table 2. Baseline characteristics and neonatal complications in preterm infants with and without the combined outcome NEC or death before 34 wks of corrected gestational age.

\begin{tabular}{|c|c|c|c|c|c|}
\hline & $\begin{array}{l}\text { NEC/death- } \\
\text { yes }(n=65)\end{array}$ & $\begin{array}{c}n \text { data } \\
\text { missing }\end{array}$ & $\begin{array}{c}\text { NEC/death-no } \\
(n=412)\end{array}$ & $\begin{array}{c}n \text { data } \\
\text { missing }\end{array}$ & $P$ value \\
\hline Birth weight (g) & 818 (SD 230) & 0 & 1032 (SD 261) & 0 & .000 \\
\hline Gestational age (wks) & 26.4 (SD 2.0) & 0 & 28.1 (SD 1.8) & 0 & .000 \\
\hline Male sex & $34(52.3)$ & 0 & $221(53.6)$ & 0 & .841 \\
\hline Prenatal steroids & $51(81.0)$ & 2 & $352(88.7)$ & 15 & .223 \\
\hline Preecclampsia & $6(9.4)$ & 1 & $65(16.0)$ & 5 & .170 \\
\hline Chorioamnionitis & $11(17.2)$ & 1 & $50(12.3)$ & 4 & .274 \\
\hline PROM & $15(23.8)$ & 2 & $109(26.8)$ & 5 & .618 \\
\hline Vaginal delivery & $34(52.3)$ & 0 & $142(34.6)$ & 2 & .006 \\
\hline Apgar (1 min) & $5[3-6]$ & 1 & $6[5-8]$ & 6 & .000 \\
\hline Apgar (5 min) & 8 [6-8] & 1 & 8 [7-9] & 6 & .000 \\
\hline RDS & $54(83.1)$ & 0 & $354(86.1)$ & 1 & .513 \\
\hline Mechanical vent. & $60(92.3)$ & 0 & $251(61.7)$ & 5 & .000 \\
\hline BPD & $24(37.5)$ & 1 & $134(32.7)$ & 2 & .447 \\
\hline Hypotension & $52(80.0)$ & 0 & $149(36.6)$ & 5 & .000 \\
\hline Sepsis & $38(60.3)$ & 2 & $212(51.7)$ & 2 & .202 \\
\hline IVH & $31(48.4)$ & 1 & $95(23.2)$ & 2 & .000 \\
\hline PVL & $5(7.8)$ & 1 & $13(3.2)$ & 3 & .072 \\
\hline PDA & $45(71.4)$ & 2 & $213(52.0)$ & 2 & .004 \\
\hline ROP & $13(25.0)$ & 13 & $85(21.6)$ & 19 & .581 \\
\hline
\end{tabular}

Results are expressed as mean (SD), median [interquartile range] or absolute numbers of patients (percentage). NEC: necrotizing enterocolitis ( $\geq$ stage II); PROM: prolonged rupture of membranes; RDS: respiratory distress syndrome; BPD: bronchopulmonary dysplasia; IVH: intraventricular hemorrhage ( $\geq$ grade 2); PVL: periventricular leukomalacia; PDA: patent ductus arteriosus; ROP: retinopathy of prematurity ( $\geq$ stage II). 


\section{ANALYSIS OF GENOTYPES}

Allele and genotype frequencies of the p.Thr1406Asn polymorphism in the total preterm population did not significantly differ from the allele and genotype frequencies observed in the population of 96 healthy term infants $(P=0.925$, Table 3$) .{ }^{30}$ The distribution of the genotypes p.Thr1406Asn did not fulfill Hardy-Weinberg criteria in the preterm population. The minor allele frequency (MAF) in the NEC group (0.208) was not significantly different from the MAF among the infants without NEC (0.303, $P=0.09$; Table 4). The MAF in the NEC group with the combined outcome NEC or death before 34 weeks $(0.200)$ was significantly different from the MAF among the infants without the outcome $(0.311, P=0.03$; Table 5$)$.

We further analyzed the effect of the CPS1 p.Thr1406Asn polymorphism on the occurrence of NEC and NEC or death under different genetic models. As shown in table 4, logistic regression analysis could not detect any significant association between the p.Thr1406Asn polymorphism and NEC in any of the genetic models. In contrast, the dominant and the additive model showed a negative significant association of the A-allele of the CPS1 p.Thr1406Asn polymorphism with the combined outcome NEC or death before 34 weeks of corrected GA (Table 5). Finally, we analyzed the correlation of the CPS1 p.Thr1406Asn polymorphism genotype with other neonatal outcomes. As shown in table 6, we could not find any significant association. 
Table 3. Distribution of the CPS1 p.Thr1406Asn polymorphism genotypes in preterm and term infants.

\begin{tabular}{lcccc}
\hline Population & CC & CA & AA & MAF \\
\hline Total study group $(n=477)$ & $248(52.0)$ & $176(36.9)$ & $53(11.1)$ & 0.296 \\
Term control group $(n=96)^{29}$ & $52(54.2)$ & $34(35.4)$ & $10(10.4)$ & 0.281 \\
\hline
\end{tabular}

Results are expressed as absolute numbers of patients (percentage).

CC denotes homozygosity for the C-encoded CPS1 $p$. Thr1406Asn polymorphism variant; $A A$ homozygosity for the A-encoded CPS1 p.Thr1406Asn polymorphism variant; $C A$ heterozygosity for CPS1 p.Thr1406Asn polymorphism; MAF: minor allele frequency. 
Table 4. Distribution of the CPS1 p.Thr1406Asn polymorphism genotypes in preterm infants with and without NEC.

\begin{tabular}{|c|c|c|c|c|c|c|c|}
\hline & Genotype & $\begin{array}{c}\text { NEC-no } \\
(n=441) \\
N(\%)\end{array}$ & $\begin{array}{c}\text { NEC-yes } \\
(n=36) \\
N(\%)\end{array}$ & OR $(95 \% \mathrm{Cl})$ & $P$-value & aOR $(95 \% \mathrm{Cl})$ & $P$ value \\
\hline \multirow[t]{3}{*}{ Codominant } & $\mathrm{CC}$ & $226(51.2)$ & $22(61.1)$ & 1 (reference) & & 1 (reference) & \\
\hline & $\mathrm{CA}$ & $163(37.0)$ & $13(36.1)$ & $0.82(0.40-1.67)$ & 0.59 & $0.87(0.41-1.88)$ & 0.73 \\
\hline & $A A$ & $52(11.8)$ & $1(2.8)$ & $0.20(0.03-1.50)$ & 0.12 & $0.21(0.03-1.66)$ & 0.14 \\
\hline \multirow[t]{2}{*}{ Dominant } & $\mathrm{CC}$ & $226(51.2)$ & $22(61.1)$ & 1 (reference) & & 1 (reference) & \\
\hline & AA_CA & $215(48.8)$ & $14(38.9)$ & $0.67(0.33-1.34)$ & 0.26 & $0.71(0.34-1.48)$ & 0.36 \\
\hline \multirow[t]{2}{*}{ Recessive } & CC_CA & $389(88.2)$ & $35(97.2)$ & 1 (reference) & & 1 (reference) & \\
\hline & AA & $52(11.8)$ & $1(2.8)$ & $0.21(0.03-1.59)$ & 0.13 & $0.23(0.03-1.71)$ & 0.15 \\
\hline Additive & & & & $0.64(0.36-1.11)$ & 0.11 & $0.66(0.36-1.19)$ & 0.17 \\
\hline MAF & & 0.303 & 0.208 & & 0.09 & & \\
\hline
\end{tabular}

OR; odds ratio; aOR: odds ratio adjusted for birth weight, gestational age, Apgar score after 1 minute, mechanical ventilation, hypotension and PDA; Cl: confidence interval; MAF: minor allele frequency; CC denotes homozygosity for the C-encoded CPS1 p.Thr1406Asn polymorphism variant; AA homozygosity for the A-encoded CPS1 p.Thr1406Asn polymorphism variant; CA heterozygosity for CPS1 p. Thr1406Asn polymorphism. 
Table 5. Distribution of the CPS1 p.Thr1406Asn polymorphism genotypes in preterm infants with and without the combined outcome NEC or death before 34 wks of corrected gestational age.

\begin{tabular}{|c|c|c|c|c|c|c|c|}
\hline & Genotype & $\begin{array}{l}\text { NEC/death-no } \\
\begin{array}{c}(n=412) \\
N(\%)\end{array}\end{array}$ & $\begin{array}{c}\text { NEC/death-yes } \\
\begin{array}{c}(n=65) \\
N(\%)\end{array}\end{array}$ & OR (95\% Cl) & $P$-value & aOR $(95 \% \mathrm{Cl})$ & $P$ value \\
\hline \multirow[t]{3}{*}{ Codominant } & $\mathrm{CC}$ & $206(50.0)$ & $42(64.6)$ & 1 (reference) & & 1 (reference) & \\
\hline & $\mathrm{CA}$ & $156(37.9)$ & $20(30.8)$ & $0.63(0.36-1.11)$ & 0.11 & $0.63(0.33-1.20)$ & 0.11 \\
\hline & AA & $50(12.1)$ & $3(4.6)$ & $0.29(0.09-0.99)$ & 0.05 & $0.28(0.08-1.02)$ & 0.05 \\
\hline \multirow[t]{2}{*}{ Dominant } & $\mathrm{CC}$ & $206(50.0)$ & $42(64.6)$ & 1 (reference) & & 1 (reference) & \\
\hline & AA_CA & $206(50.0)$ & $23(35.4)$ & $0.55(0.32-0.94)$ & 0.03 & $0.54(0.29-0.99)$ & 0.04 \\
\hline \multirow[t]{2}{*}{ Recessive } & CC_CA & $362(87.9)$ & $62(95.4)$ & 1 (reference) & & 1 (reference) & \\
\hline & $A A$ & $50(12.1)$ & $3(4.6)$ & $0.35(0.11-1.16)$ & 0.09 & $0.33(0.10-1.18)$ & 0.09 \\
\hline Additive & & & & $0.59(0.38-0.91)$ & 0.02 & $0.58(0.36-0.93)$ & 0.03 \\
\hline MAF & & 0.311 & 0.200 & & 0.03 & & \\
\hline
\end{tabular}

OR; odds ratio; aOR:odds ratio adjusted for birth weight, gestational age, Apgar score after 1 minute, Apgar score after 5 minute, mechanical ventilation, hypotension and PDA; Cl: confidence interval; MAF: minor allele frequency; CC denotes homozygosity for the C-encoded CPS1 p. Thr1406Asn polymorphism variant; AA homozygosity for the A-encoded CPS1 p.Thr1406Asn polymorphism variant; CA heterozygosity for CPS1 p.Thr1406Asn polymorphism. 
Table 6. Distribution of the CPS1 p.Thr1406Asn polymorphism genotypes in several neonatal outcomes.

\begin{tabular}{|c|c|c|c|c|c|c|}
\hline Outcome & & $\begin{array}{l}\text { No. of } \\
\text { infants }\end{array}$ & CC & CA & AA & $\begin{array}{c}P \\
\text { value }\end{array}$ \\
\hline $\begin{array}{l}\text { Gestational age } \\
\text { (wks) }\end{array}$ & & 477 & 27.7 (SD 2.0) & 28.0 (SD 1.7) & 28.1 (SD 1.9) & 0.16 \\
\hline Birth weight (g) & & 477 & 984 (SD 269) & 1018 (SD 260) & 1043 (SD 277) & 0.23 \\
\hline \multirow[t]{2}{*}{ RDS } & yes & 408 & 205 (50.3) & $158(38.7)$ & $45(11.0)$ & 0.14 \\
\hline & no & 68 & $42(61.7)$ & $18(26.5)$ & $8(11.8)$ & \\
\hline \multirow[t]{2}{*}{ BPD } & yes & 158 & 89 (56.3) & $53(33.5)$ & $16(10.1)$ & 0.39 \\
\hline & no & 316 & $157(49.7)$ & $122(38.6)$ & $37(11.7)$ & \\
\hline BPD or death & yes & 187 & $109(58.3)$ & $59(31.6)$ & $19(10.2)$ & 0.07 \\
\hline before 36 weeks & no & 288 & $137(47.6)$ & $117(40.6)$ & $34(11.8)$ & \\
\hline \multirow[t]{2}{*}{ Hypotension } & yes & 201 & $103(51.2)$ & 77 (38.3) & $21(10.4)$ & 0.84 \\
\hline & no & 271 & $141(52.0)$ & $98(36.2)$ & $32(11.8)$ & \\
\hline \multirow[t]{2}{*}{ Sepsis } & yes & 250 & $132(52.8)$ & $86(34.4)$ & $32(12.8)$ & 0.32 \\
\hline & no & 223 & $113(50.7)$ & 89 (39.9) & $21(9.4)$ & \\
\hline \multirow[t]{2}{*}{ IVH } & yes & 126 & $68(54.0)$ & $42(33.3)$ & $16(12.7)$ & 0.53 \\
\hline & no & 348 & $178(51.1)$ & $134(38.5)$ & $36(10.3)$ & \\
\hline \multirow[t]{2}{*}{ PVL } & yes & 18 & $12(66.7)$ & $3(16.7)$ & $3(16.7)$ & 0.18 \\
\hline & no & 455 & $233(51.2)$ & $173(38.0)$ & $49(10.8)$ & \\
\hline \multirow[t]{2}{*}{ PDA } & yes & 258 & $144(55.8)$ & 86 (33.3) & $28(10.9)$ & 0.14 \\
\hline & no & 215 & $101(47.0)$ & $89(41.4)$ & $25(11.6)$ & \\
\hline \multirow[t]{2}{*}{ ROP } & yes & 98 & $55(56.1)$ & 33 (33.7) & $10(10.2)$ & 0.54 \\
\hline & no & 347 & 173 (49.9) & $132(38.0)$ & $42(12.1)$ & \\
\hline ROP or death & yes & 129 & $76(58.9)$ & $40(31.0)$ & $13(10.1)$ & 0.13 \\
\hline before screening & no & 324 & $157(48.5)$ & $128(39.5)$ & $39(12.0)$ & \\
\hline
\end{tabular}

Results are expressed as absolute numbers of patients (percentage) or mean (SD).

CC denotes homozygosity for the C-encoded CPS1 $\mathrm{p}$. Thr1406Asn polymorphism variant; $A A$ homozygosity for the A-encoded CPS1 p.Thr1406Asn polymorphism variant; CA heterozygosity for CPS1 p.Thr1406Asn polymorphism. RDS: respiratory distress syndrome; BPD: bronchopulmonary dysplasia; IVH: intraventricular hemorrhage ( $\geq$ grade 2); PVL: periventricular leukomalacia; PDA: patent ductus arteriosus; ROP: retinopathy of prematurity ( $\geq$ stage II). 


\section{DISCUSSION}

This is one of the largest prospective studies investigating the association of a SNP with NEC. Our study could not detect a significant association between the p.Thr1406Asn genotype of the CPS1 gene and the risk of developing NEC in very preterm infants ( $G A \leq 30$ weeks and $B W \leq 1500 \mathrm{~g}$ ). However, when the combined outcome NEC or death before 34 weeks of corrected GA was analysed, it was observed that the minor A-allele of the p.Thr1406Asn polymorphism was significantly less frequent in the group of infants who developed NEC or died than in those who survived without NEC. In addition, a significant negative association of the A-allele with the combined outcome NEC or death was found using the dominant and the additive genetic models.

In our previous retrospective case-control study, we examined the relationship between the CPS1 p.Thr1406Asn polymorphism and the presence of NEC in preterm infants and we found that patients with NEC showed an overrepresentation of the Cencoded variant of the CPS1. ${ }^{27}$ Consequently, the A-encoded variant of the p.Thr1406Asn polymorphism was underrepresented in the infants with NEC. However, although the cases and the controls were well matched for GA and BW, when adjusted for these two known risk factors for NEC, the association between NEC and the p.Thr1406Asn polymorphism did not remain significant. ${ }^{27}$ In the present prospective cohort study, we observed that the A-allele was less frequent in the infants with NEC in a proportion close to reach statistical significance $(P=0.09)$. Moreover, only one infant with NEC was homozygous for the A-allele. Nevertheless, none of the genetic models could demonstrate a statistically significant association between the p.Thr1406Asn genotype and NEC.

An important issue in designing studies involving high-risk patients is the selection of an appropriate primary outcome when death is a competing outcome. ${ }^{34}$ In this situation, some patients will die before the outcome of interest can occur. For this reason, a composite outcome including death is often used when complications of prematurity such as BPD or NEC are studied. ${ }^{34}$ Since the onset of 'classical' NEC takes place around the end of the third week of life ${ }^{28,29}$, we performed an additional 
analysis in which the infants with NEC were combined with the infants who died before 34 weeks of corrected GA. Interestingly, we observed that the minor A-allele was significantly less frequent among the infants with the combined outcome NEC or death. Moreover, the dominant and additive model demonstrated that the $A$ variant of the p.Thr1406Asn polymorphism significantly decreased the risk of developing the combined outcome of NEC or death before 34 weeks of corrected GA. Thus, in other words, the minor A variant of the polymorphism might protect against this combined outcome.

In accordance with our results, it has been reported a protective role of the A-allele toward persistent pulmonary hypertension of the newborn ${ }^{15}$, pulmonary hypertension following surgical repair of congenital heart defects ${ }^{26}$, and hepatic veno-occlusive disease after bone marrow transplantation ${ }^{23}$. Moreover, the haplotype formed by the SNPs of CPS1 rs715 and rs1047891 (p.Thr1406Asn) yield a protective association with decreased risk of coronary artery disease in women ${ }^{35}$. As mentioned in the introduction, those findings led to the speculation that individuals with the A-allele may have an advantage in terms of availability of the NOS substrate L-arginine, especially under conditions of environmental stress. ${ }^{15,23,26}$ Accordingly, the serum levels of arginine were higher in term newborns carrying the AA genotype. ${ }^{15}$ However, activity reports on the in vitro activity of the p.Thr1406Asn variants are contradictory. ${ }^{22}$ Thus, natural (A-encoded) Asn1406 CPS has been reported to have higher enzymatic activity than the (C-encoded) Thr1406 variant ${ }^{15,23,26}$ but recombinant Asn1406 CPS1 showed inferior catalytic properties. ${ }^{36}$ In order to evaluate whether p.Thr1406Asn genotypes correlated with urea cycle intermediates levels in preterm infants, we measured the concentrations of arginine and citrulline in the first 128 infants included in the present cohort and we did not observe any significant differences. ${ }^{30}$ This lack of effect of CPS1 genotype on L-arginine concentrations has been also reported in adults. ${ }^{25}$ Nevertheless, one limitation of our study was that L-arginine levels were determined between 6 and 12 hours after birth, whereas NEC has its onset later in life. ${ }^{28,29}$ It can be speculated that the alterations in arginine levels related to the p.Thr1406Asn polymorphism may be only relevant under the stress conditions generated around the time of NEC onset. At that moment, being carrier of a genetic variant that potentially increases NO production (i.e., the A-allele) might be of critical relevance because NO 
is a key regulator intestinal blood flow, protector of the mucosa, and modulator of the inflammatory response. ${ }^{10-12}$ In addition, the infants homozygous for the C-allele might be more susceptible to NEC and arginine supplementation ${ }^{16,20,21}$ might be particularly indicated in this group.

One limitation of our study is that we did not collect information on the feeding practices. Human milk protects against $\mathrm{NEC}^{1-3}$ and, although the preterm formulas currently used have concentrations of arginine similar to the human milk ${ }^{16}$, arginine intake might be different depending on the infants diet. Plasma arginine levels are likely to represent a balance between arginine intake, arginine synthesis, and the demands of protein synthesis and the multiple metabolic pathways for arginine utilization. ${ }^{14,37}$ Enteric arginine synthesis appears to be necessary to cover neonatal requirements, because mammalian milk is a relatively poor source of arginine, whereas its precursors proline and glutamine are abundant. ${ }^{38}$ In fact, proline is the major contributor to arginine synthesis in human preterm infants. ${ }^{39} \mathrm{CPS}$, ornithine aminotransferase, and argininosuccinate synthetase are key enzymes in the control of de novo intestinal synthesis of arginine, which are already expressed in the mid gestation human intestine. ${ }^{38}$ However, hypoargininemia often develops in preterm infants, in particular if they are maintained on total parenteral nutrition, and it has been suggested that the intestine only produces arginine if substrate is supplied through enteral nutrition. ${ }^{14,37-39}$ In situations of reduced availability of the substrate L-arginine or the cofactor $\mathrm{BH}_{4}$, NOS enzymatic activity becomes uncoupled, resulting in the production of superoxide instead of NO. ${ }^{40}$ Therefore, besides resulting in a paucity of NO, the uncoupled enzyme will generate free radicals resulting in further intestinal damage. In addition, it should be taken into account that, besides NO, arginine is a substrate for synthesis of many biologically important molecules including agmatine, polyamines, and creatine..$^{14,38,41}$ These metabolites have roles in energy metabolism, gene expression, apoptosis, and cell proliferation and differentiation, which are crucial in intestinal homeostasis. . $^{14,38,41}$ Our present results suggest that functional genetic variations in the CPS enzyme might be, at least partially, the link between hypoarginimemia and NEC in preterm infants. ${ }^{14,16-19}$ Alternatively, recent experimental evidences highlighted the role of argininosuccinate lyase, another enzyme involved in the intestinal synthesis of arginine, in the pathogenesis of NEC. ${ }^{41}$ 


\section{CONCLUDING REMARKS}

NEC affects only a minority of preterm infants, which suggest an individual susceptibility toward the disease. Genetic polymorphisms might be an important factor in this individual susceptibility. 2,4,5,9,42-51 Our study provides further evidence that a functional variant of the CPS1 gene may contribute to NEC susceptibility. Nevertheless, NEC is a complex multifactorial disease and an isolated genetic derangement may not be sufficient to account for the entire spectrum of its pathophysiology.

Given the importance of prematurity, intestinal function, immune defense, inflammatory signaling, and microcirculatory regulation mechanisms, potential variations in many genes could protect or leave a host infant susceptible to NEC. 5,9,4251 Future studies investigating the association of multiple SNPs and NEC may allow for the development of a laboratory genetic test that could predict, when environmental factors are properly assessed, the risk/probability of preterm infants developing NEC and lead to more targeted therapies. 


\section{REFERENCES}

1 Neu, J. \& Walker, W. A. Necrotizing enterocolitis. N Engl J Med 364, 255-264 (2011).

2 Neu, J. Necrotizing enterocolitis: the mystery goes on. Neonatology 106, 289295 (2014).

3 Wu, S. F., Caplan, M. \& Lin, H. C. Necrotizing enterocolitis: old problem with new hope. Pediatr Neonatol 53, 158-163 (2012).

4 Treszl, A., Tulassay, T. \& Vasarhelyi, B. Genetic basis for necrotizing enterocolitis--risk factors and their relations to genetic polymorphisms. Front Biosci 11, 570-580 (2006).

$5 \mathrm{Ng}, \mathrm{P}$. C. Biomarkers of necrotising enterocolitis. Semin Fetal Neonatal Med 19, 33-38 (2014).

6 Lim, J. C., Golden, J. M. \& Ford, H. R. Pathogenesis of neonatal necrotizing enterocolitis. Pediatr Surg Int 31, 509-518 (2015).

7 Gordon, P. V., Swanson, J. R., Attridge, J. T. \& Clark, R. Emerging trends in acquired neonatal intestinal disease: is it time to abandon Bell's criteria? $J$ Perinatol 27, 661-671 (2007).

8 Berman, L. \& Moss, R. L. Necrotizing enterocolitis: an update. Semin Fetal Neonatal 16, 145-150 (2011).

9 Lin, P. W., Nasr, T. R. \& Stoll, B. J. Necrotizing enterocolitis: recent scientific advances in pathophysiology and prevention. Semin Perinato/32, 70-82 (2008).

10 Reber, K. M., Nankervis, C. A. \& Nowicki, P. T. Newborn intestinal circulation. Physiology and pathophysiology. Clin Perinatol 29, 23-39 (2002).

11 Upperman, J. S. et al. Mechanisms of nitric oxide-mediated intestinal barrier failure in necrotizing enterocolitis. Semin Pediatr Surg 14, 159-166 (2005).

12 Rhoads, J. M. et al. Arginine stimulates intestinal cell migration through a focal adhesion kinase dependent mechanism. Gut 53, 514-522 (2004).

13 Wu, G. Amino acids: metabolism, functions, and nutrition. Amino Acids 37, 117 (2009).

14 Wu, G., Jaeger, L. A., Bazer, F. W. \& Rhoads, J. M. Arginine deficiency in preterm infants: biochemical mechanisms and nutritional implications. J Nutr Biochem 15, 442-451 (2004). 
15 Pearson, D. L. et al. Neonatal pulmonary hypertension--urea-cycle intermediates, nitric oxide production, and carbamoyl-phosphate synthetase function. N Engl J Med 344, 1832-1838 (2001).

16 Amin, H. J. et al. Arginine supplementation prevents necrotizing enterocolitis in the premature infant. J Pediatr 140, 425-431 (2002).

17 Becker, R. M. et al. Reduced serum amino acid concentrations in infants with necrotizing enterocolitis. J Pediatr 137, 785-793 (2000).

18 Zamora, S. A. et al. Plasma L-arginine concentrations in premature infants with necrotizing enterocolitis. J Pediatr 131, 226-232 (1997).

19 Richir, M. C. et al. Low plasma concentrations of arginine and asymmetric dimethylarginine in premature infants with necrotizing enterocolitis. Br J Nutr 97 , 906-911 (2007).

20 Polycarpou, E. et al. Enteral L-Arginine Supplementation for Prevention of Necrotizing Enterocolitis in Very Low Birth Weight Neonates A Double-Blind Randomized Pilot Study of Efficacy and Safety. JPEN J Parenter Enteral Nutr, 0148607112471561 (2013).

21 Mitchell, K. et al. Arginine supplementation in prevention of necrotizing enterocolitis in the premature infant: an updated systematic review. BMC Pediatr 14, 1 (2014).

22 Martinez, A. I., Perez-Arellano, I., Pekkala, S., Barcelona, B. \& Cervera, J. Genetic, structural and biochemical basis of carbamoyl phosphate synthetase 1 deficiency. Mol Genet Metab 01, 311-323 (2010).

23 Summar, M. L. et al. Environmentally determined genetic expression: clinical correlates with molecular variants of carbamyl phosphate synthetase I. $\mathrm{Mol}$ Genet Metab 81 Suppl 1, S12-19 (2004).

24 Summar, M. L. et al. Characterization of genomic structure and polymorphisms in the human carbamyl phosphate synthetase I gene. Gene 311, 51-57 (2003).

25 Summar, M. L. et al. Relationship between carbamoyl-phosphate synthetase genotype and systemic vascular function. Hypertension 43, 186-191 (2004).

26 Canter, J. A. et al. Genetic variation in the mitochondrial enzyme carbamylphosphate synthetase I predisposes children to increased pulmonary artery pressure following surgical repair of congenital heart defects: a validated genetic association study. Mitochondrion 7, 204-210 (2007). 
27 Moonen, R. M. et al. Carbamoyl phosphate synthetase polymorphisms as a risk factor for necrotizing enterocolitis. Pediatr Res 62, 188-190 (2007).

28 Yee, W. H. et al. Incidence and timing of presentation of necrotizing enterocolitis in preterm infants. Pediatrics 129, e298-304 (2012).

29 Llanos, A. R. et al. Epidemiology of neonatal necrotising enterocolitis: a population-based study. Paediatr Perinat Epidemiol 16, 342-349 (2002).

30 Moonen, R. M. et al. The T1405N carbamoyl phosphate synthetase polymorphism does not affect plasma arginine concentrations in preterm infants. PloS one 5, e10792 (2010).

31 Jobe, A. H. \& Bancalari, E. Bronchopulmonary dysplasia. Am J Respir Crit Care Med 163, 1723-1729 (2001).

32 Papile, L. A., Burstein, J., Burstein, R. \& Koffler, H. Incidence and evolution of subependymal and intraventricular hemorrhage: a study of infants with birth weights less than 1,500 gm. J Pediatr 92, 529-534 (1978).

33 Clarke, G. M. et al. Basic statistical analysis in genetic case-control studies. Nat Protoc 6, 121-133 (2011).

34 Das, A. et al. in Seminars in Perinatology. (Elsevier).

35 Hartiala, J. A. et al. Genome-wide association study and targeted metabolomics identifies sex-specific association of CPS1 with coronary artery disease. Nat Commun 7, 10558 (2016).

36 Ahuja, V. \& Powers-Lee, S. Human carbamoyl-phosphate synthetase: insight into $\mathrm{N}$-acetylglutamate interaction and the functional effects of a common single nucleotide polymorphism. J Inherit Metab Dis 31, 481-491 (2008).

37 Morgan, C. \& Burgess, L. High protein intake does not prevent low plasma levels of conditionally essential amino acids in very preterm infants receiving parenteral nutrition. JPEN J Parenter Enteral Nutr, 0148607115594009 (2015).

38 Kohler, E. S. et al. The human neonatal small intestine has the potential for arginine synthesis; developmental changes in the expression of argininesynthesizing and -catabolizing enzymes. BMC Dev Biol 8, 107 (2008).

39 Tomlinson, C., Rafii, M., Sgro, M., Ball, R. O. \& Pencharz, P. Arginine is synthesized from proline, not glutamate, in enterally fed human preterm neonates. Pediatr Res 69, 46-50 (2011). 
40 Sullivan, J. C. \& Pollock, J. S. Coupled and uncoupled NOS: separate but equal? Uncoupled NOS in endothelial cells is a critical pathway for intracellular signaling. Circ Res 98, 717-719 (2006).

41 Premkumar, M. H. et al. Argininosuccinate lyase in enterocytes protects from development of necrotizing enterocolitis. Am J Physiol Gastrointest Liver Physiol 307, G347-354 (2014).

42 Franklin, A. L. et al. Are Immune Modulating Single Nucleotide Polymorphisms Associated with Necrotizing Enterocolitis? Sci Rep 5, 18369 (2015).

43 Sampath, V. et al. SIGIRR Genetic Variants in Premature Infants With Necrotizing Enterocolitis. Pediatrics 135, e1530-1534 (2015).

44 Zhou, W. et al. Association of neonatal necrotizing enterocolitis with myeloid differentiation-2 and GM2 activator protein genetic polymorphisms. Mol Med Rep 12, 974-980 (2015).

45 Heninger, E. et al. Genetic variants of the interleukin-18 promoter region (-607) influence the course of necrotising enterocolitis in very low birth weight neonates. Eur J Pediatr 161, 410-411 (2002).

46 Bokodi, G., Derzbach, L., Banyasz, I., Tulassay, T. \& Vasarhelyi, B. Association of interferon gamma $\mathrm{T}+874 \mathrm{~A}$ and interleukin $12 \mathrm{p} 40$ promoter CTCTAA/GC polymorphism with the need for respiratory support and perinatal complications in low birthweight neonates. Arch Dis Child Fetal Neonatal Ed 92, F25-29 (2007).

47 Prencipe, G. et al. Association between mannose-binding lectin gene polymorphisms and necrotizing enterocolitis in preterm infants. J Pediatr Gastroenterol Nutr 55, 160-165 (2012).

48 Sampath, V. et al. The NFKB1 (g.-24519delATTG) variant is associated with necrotizing enterocolitis (NEC) in premature infants. J Surg Res 169, e51-57 (2011).

49 Treszl, A. et al. Lower prevalence of IL-4 receptor alpha-chain gene G variant in very-low-birth-weight infants with necrotizing enterocolitis. J Pediatr Surg 38, 1374-1378 (2003). 
50 Henderson, G. et al. Cytokine gene polymorphisms in preterm infants with necrotising enterocolitis: genetic association study. Arch Dis Child Fetal Neonatal Ed 94, F124-128 (2009).

51 Bányász, I. et al. Genetic polymorphisms for vascular endothelial growth factor in perinatal complications. Eur Cytokine Netw 17, 266-270 (2006).

Acknowledgments: Supported by a grant from "Fundación de Investigación Médica Mutua Madrileña". The funder had no role in study design, data collection and analysis, decision to publish, or preparation of the manuscript. 
Chapter 7

THE T1405N CARBAMOYL PHOSPHATE SYNTHETASE POLYMORPHISM DOES NOT AFFECT PLASMA ARGININE CONCENTRATIONS IN PRETERM INFANTS

Rob M. J. Moonen, Iballa Reyes, Giacomo Cavallaro, Gema González-Luis, Jaap A.

Bakker, Eduardo Villamor

PLoS One 25, e10792 (2010)

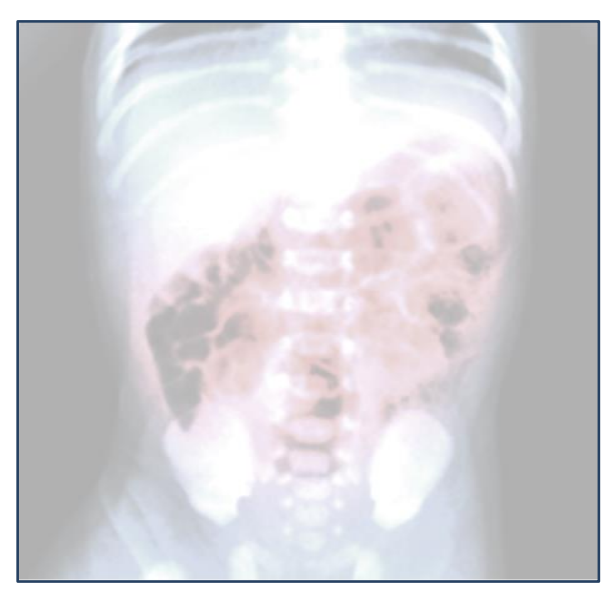




\section{ABSTRACT}

Background: A C-to-A nucleotide transversion (T1405N) in the gene that encodes carbamoyl-phosphate synthetase 1 (CPS1) has been associated with changes in plasma concentrations of L-arginine in term and near term infants but not in adults. In preterm infants homozygosity for the CPS1 Thr1405 variant (CC genotype) was associated with an increased risk of having necrotizing enterocolitis (NEC). Plasma Larginine concentrations are decreased in preterm infants with NEC.

Aim: To examine the putative association between the CPS1 T1405N polymorphism and plasma arginine concentrations in preterm infants.

Methods: Prospective multicenter cohort study. Plasma and DNA samples were collected from 128 preterm infants (<30 weeks) between 6 and 12 hours after birth. Plasma amino acid and CPS1 T1405N polymorphism analysis were performed.

Results: Distribution of genotypes did not differ between the preterm (CC:CA:AA = 55.5\%:33.6\%:10.9\%, $n=128$ ) and term infants (CC:CA:AA $=54.2 \%: 35.4 \%: 10.4 \%$, $n=96)$. There was no association between the CPS1 genotype and plasma L-arginine or L-citrulline concentration, or the ornithine to citrulline ratio, which varies inversely with CPS1 activity. Also the levels of asymmetric dimethylarginine, and symmetric dimethylarginine were not significantly different among the three genotypes.

Conclusions: The present study in preterm infants did not confirm the earlier reported association between CPS1 genotype and L-arginine levels in term infants. 


\section{INTRODUCTION}

Necrotizing enterocolitis (NEC) remains a leading cause of morbidity and mortality in neonatal intensive care units. Although several predisposing factors have been identified, the exact etiology of NEC is unknown. Suspected pathophysiological mechanisms include the developmental immaturity of gastrointestinal motility, digestive ability, circulatory regulation, intestinal barrier function, and immune defence. ${ }^{1-5}$ In the last years, numerous research efforts focused on identifying biomarkers for infants at risk for NEC. 6,7

Nitric oxide (NO) has attracted considerable attention in the pathophysiology of NEC as it pertains to the regulation of intestinal blood flow and plays a key role in the maintenance of mucosal integrity, intestinal barrier function, and post-injury intestinal reparation. ${ }^{8-12} \mathrm{NO}$ is generated by NO synthase (NOS) during the enzymatic conversion of $\mathrm{L}$-arginine to $\mathrm{L}$-citrulline. The methylated arginine metabolite asymmetric dimethylarginine (ADMA) competitively inhibits L-arginine uptake of endothelial cells and NOS activity. ${ }^{13,14}$ ADMA has emerged as an early marker and/or mediator of endothelial dysfunction and it has been proved to be a novel, independent risk factor of cardiovascular and metabolic diseases. ${ }^{15}$

The NOS substrate L-arginine is an essential amino acid for young mammals. 16,17 Metabolic and molecular studies indicate that the underdevelopment of intestinal arginine synthesis may be primarily responsible for remarkably low plasma arginine concentrations in preterm neonates. ${ }^{17-18}$ Several studies demonstrated that plasma arginine concentrations are even more decreased in premature infants with NEC. ${ }^{14,17,19-21}$ Moreover, arginine supplementation reduced the incidence of NEC in one small randomized controlled study. ${ }^{19}$

The first step arginine formation occurs inside the mitochondrion and is catalyzed by the enzyme, carbamyl-phosphate synthetase I (CPS1). A specific single nucleotide polymorphism designated as $T 1405 \mathrm{~N}$ in the CPS1 gene results in a threonine to asparagine amino acid substitution at an important cofactor binding site. Previous studies demonstrated the association of the CPS1 T1405N genotype with clinical 
situations where endogenous NO production is critically important, such as neonatal pulmonary hypertension, increased pulmonary artery pressure following surgical repair of congenital heart defects, or hepatovenocclusive disease following bone marrow transplantation. ${ }^{18,22,23}$

Recently, we observed in a retrospective case-control study that homozygosity for the CPS1 Thr1405 variant (CC T1405N genotype) was associated with an increased risk of having NEC. ${ }^{24}$ This corresponded with the observation that term newborns with the CC T1405N genotype had significantly lower levels of $L$-arginine and NO metabolites than infants with the AA T1405N genotype (homozygous for the Asn1405 variant). ${ }^{18}$ However this association between CPS1 T1405N genotype and L-arginine levels was not present in adults ${ }^{22}$ and has not been yet studied in preterm infants. In the present work, we hypothesized that the CPS1 T1405N genotype would influence the plasmatic concentrations of L-arginine, L-citrulline, and methylated arginine metabolites in preterm infants shortly after birth. 


\section{METHODS}

\section{PATIENTS AND STUDY DESIGN.}

Between July 2007 and October 2008 we performed a prospective multicenter cohort study. All infants with a gestational age $<30$ weeks and birth weight $<1500 \mathrm{~g}$ born in this period and admitted to the level III neonatal intensive care unit of the Maastricht University Medical Center (The Netherlands), Hospital Universitario Materno-Infantil de Canarias (Las Palmas de Gran Canaria, Spain), and Carlo Poma Hospital (Mantova, Italy) were eligible for participation in the study, which was approved the Local Research Ethics Committees of the participating centres and registered in ClinicalTrials.gov Protocol Registration System (NCT00554866). Written informed consent from the parents was obtained. Exclusion criteria were the following: blood transfusion, enteral or parenteral protein intake, or inhaled NO administration before blood sampling. Therefore, none of the included infants received enteral nutrition and parenteral nutrition consisted only of dextrose and electrolytes. In addition, none of the included infants received insulin treatment before or during sampling. One blood sample $(500 \mu \mathrm{L})$ was obtained between 6 and 12 hours after birth from an umbilicalartery or peripheral artery catheter. When not available, the blood sample was obtained from venous puncture. Immediately after collection, heparinized blood samples were put on ice and centrifuged within 10 minutes $\left(4000 \mathrm{rpm}, 10 \mathrm{~min}, 4^{\circ} \mathrm{C}\right.$ ) to obtain plasma. The plasma was deproteinised with $6 \mathrm{mg}$ of solid 5-sulphosalicylic acid (SSA, Sigma, St. Louis, MO) per $100 \mu \mathrm{L}$ plasma, and stored at $-80^{\circ} \mathrm{C}$ until further analysis. Buccal cell samples for DNA testing were obtained with a sterile OmniSwab (Whatman, Sanford, ME), collected in Eppendorf sterile PCR tubes, and stored at $-80^{\circ} \mathrm{C}$ until further analysis. The samples obtained in Las Palmas and Mantova were transported on dry ice to Maastricht where all the analysis were performed. Data on clinical characteristics were retrieved from medical records. To provide some data about the genotype distribution of the general population in the Netherlands, Spain and Italy, bucall cell samples were obtained from a group of healthy term infants. 


\section{ANALYSIS OF THE T1405N POLYMORPHISM IN THE CPS1 GENE}

DNA was extracted using standard methods and stored at $-20^{\circ} \mathrm{C}$ until genotyping. A 214-bp fragment encompassing the $4332 \mathrm{C}>\mathrm{A}$ polymorphism in exon 36 of the CPS1 gene was amplified using polymerase chain reaction (PCR). Primers used were (forward) GCM357 5'-TAAATGCAGCTGTTTG CCAC-3' and (reverse) GCM358 5'GACTTGCAATCAAGTAAGGTGAAA-3'. The PCR mix consisted of 1X GeneAmp PCR Buffer II (Perkin-Elmer, Branchburg, NJ), $0.2 \mathrm{mM}$ deoxyribonucleoside triphosphate (Pharmacia Biotech, Bridgewater, NJ), $1.5 \mathrm{mM} \mathrm{MgCl} 2$ (Perkin-Elmer, Branchburg, NJ), $250 \mathrm{nM}$ of both primers, and $0.025 \mathrm{U} / \mu \mathrm{L}$ of AmpliTaq Gold (PerkinElmer, Branchburg, NJ). Thermocycling conditions started with an initial denaturation of $10 \mathrm{~min} 95^{\circ} \mathrm{C}$, followed by 35 cycles of $95^{\circ} \mathrm{C}(45 \mathrm{~s}), 55^{\circ} \mathrm{C}(45 \mathrm{~s}), 72^{\circ} \mathrm{C}(45 \mathrm{~s})$, and ended with a final extension step of $10 \mathrm{~min}$ at $72^{\circ} \mathrm{C}$. The PCR product was purified and directly sequenced using the reverse primer.

\section{PLASMA AMINO ACID ANALYSIS}

Plasma amino acid concentrations were analyzed by high-performance liquid chromatography as previously described. ${ }^{25}$ Dimethylargines, ADMA and SDMA, were determined in plasma using an ultra-performance liquid chromatography (UPLC) separation module coupled to an electrospray ionisation tandem mass spectrometry (ESI-MS/MS, Quattro Premier, Waters, Etten-Leur, The Netherlands). Separation of the components of interest was adapted from the described method for the determination of amino acids. ${ }^{26}$ Briefly, plasma samples were mixed with stable isotope labelled ADMA and deproteinised with SSA and diluted. ADMA and SDMA were detected in the multiple reaction mode (MRM) in ESI positive mode. ${ }^{27}$

\section{STATISTICS}

Assumptions of sample size were based on on data provided by Pearson et al, Summar et al and Moonen et al. ${ }^{18,22,24}$ Sample size was calculated to detect a difference of $10 \mu \mathrm{mol} / \mathrm{L}$ (SD 8) in mean L-arginine concentration between the $A A$ and the $\mathrm{CC}$ genotypes. We found that we needed 11 subjects per group to detect this difference. Assuming a $10-12 \%$ incidence of the AA genotype $22,24,92-110$ patients would be necessary to include the 11 patients with AA genotype. 
To determine whether polymorphisms of the CPS1 gene were in Hardy-Weinberg equilibrium, the frequencies of alleles and of genotypes were analyzed, and actual and predicted genotype frequencies were compared by $x^{2}$ analysis with one degree of freedom. Results for continuous variables are expressed as mean (SD) or, if variables were not normally distributed, as median (interquartile range). Differences between mean values were assessed by one-way ANOVA followed by Bonferroni's post hoc $\mathrm{t}$ test, t-test or the Mann-Whitney $U$ test, as appropriate. Differences were considered significant at a $P<0.05$. All analyses were performed using GraphPad Prism (version 5.00 for Windows, GraphPad Software, San Diego California USA). 


\section{RESULTS}

In total 128 preterm infants were enrolled in the study. Of these 128 infants, 31 were enrolled in Maastricht, 66 in Las Palmas de Gran Canaria and 31 in Mantova. The clinical characteristics of the patients are summarized in Table 1. Bucall cell samples were obtained from 96 healthy term infants (25 in Maastricht, 31 in Las Palmas de Gran Canaria and 40 in Mantova).

Table 1. Baseline patient characteristics

\begin{tabular}{|c|c|c|c|c|c|}
\hline & $\begin{array}{l}\text { Total study } \\
\text { group } \\
\quad(n=128)\end{array}$ & $\begin{array}{c}C C \\
(n=71)\end{array}$ & $\begin{array}{c}C A \\
(n=43)\end{array}$ & $\begin{array}{c}\text { AA } \\
(n=14)\end{array}$ & $\begin{array}{c}P \\
\text { value }\end{array}$ \\
\hline $\begin{array}{l}\text { Antenatal } \\
\text { corticosteroids }\end{array}$ & $101(79)$ & $58(82)$ & $31(72)$ & $12(86)$ & NS \\
\hline C-section & $83(65)$ & $49(69)$ & $28(65)$ & $6(43)$ & NS \\
\hline $\begin{array}{l}\text { Gestational } \\
\text { age (wks) }\end{array}$ & $\begin{array}{c}28.1 \\
{[27.3-29.6]}\end{array}$ & $\begin{array}{c}28.1 \\
{[27.4-29.6]}\end{array}$ & $\begin{array}{c}28.1 \\
{[27.2-29.1]}\end{array}$ & $\begin{array}{c}29.1 \\
{[26.4-29.7]}\end{array}$ & NS \\
\hline Birth weight $(\mathrm{g})$ & $\begin{array}{c}1037 \\
(S D 241)\end{array}$ & $\begin{array}{c}1038 \\
(S D 250)\end{array}$ & $\begin{array}{c}1028 \\
(S D 229)\end{array}$ & $\begin{array}{c}1059 \\
(S D 252)\end{array}$ & NS \\
\hline Male sex & $76(59)$ & $41(58)$ & $28(65)$ & $7(50)$ & NS \\
\hline $\begin{array}{l}\text { Death before } \\
\text { discharge }\end{array}$ & $12(9)$ & $9(13)$ & $3(7)$ & $0(0)$ & NS \\
\hline CRIB score & $2[1-5]$ & $2[1-5]$ & $2[1-4]$ & $2[1-6]$ & NS \\
\hline
\end{tabular}

Results are expressed as mean (SD), median [interquartile range], or absolute numbers of patients (percentage). Statistical analyses between the different genotypes. NS $=$ not significant. 
The distribution of the CPS1 genotypes for the polymorphism at position 4332 within the overall study population fulfilled Hardy-Weinberg criteria. Genotype distribution (CC denotes homozygosity for the C-encoded Thr1405 variant, AA homozygosity for the A-encoded Asn1405 variant and $A C$ heterozygosity for this polymorphism at position 1405) in preterm infants (CC:CA:AA = 55.5\%:33.6\%:10.9\%, $n=128$ ) (Table 2) did not significantly differ from genotype distribution in term infants (CC:CA:AA = $54.2 \%: 35.4 \%: 10.4 \%, n=96$ ) (Table 3 ). No significant differences among the three centers were observed in genotype distribution in preterm (Table 2) or term (Table 3) infants. As shown in Table 1, there were no significant differences in the clinical characteristics among the three CPS1 genotypes.

Table 2. Distribution of the CPS1 genotypes for the polymorphism at position 1405 in preterm infants

\begin{tabular}{lccc}
\hline & CC & CA & AA \\
\hline Las Palmas de Gran Canaria (Spain), $\mathrm{n}=66$ & $39(59.1)$ & $19(28.8)$ & $8(12.1)$ \\
Mantova (Italy), $\mathrm{n}=31$ & $18(58.1)$ & $12(38.7)$ & $1(3.2)$ \\
Maastricht (the Netherlands), $\mathrm{n}=31$ & $14(45.2)$ & $12(38.7)$ & $5(16.1)$ \\
Total study group, $\mathrm{n}=128$ & $71(55.5)$ & $43(33.6)$ & $14(10.9)$ \\
\hline
\end{tabular}

Results are expressed as absolute numbers of patients (percentage). CC denotes homozygosity for the C-encoded Thr1405 variant, AA homozygosity for the A-encoded Asn 1405 variant, and AC heterozygosity for this polymorphism. 
Table 3. Distribution of the CPS1 genotypes for the polymorphism at position 1405 in healthy term infants

\begin{tabular}{lccc}
\hline & CC & CA & AA \\
\hline Las Palmas de Gran Canaria (Spain), $\mathrm{n}=31$ & $19(61.3)$ & $7(22.6)$ & $5(16.1)$ \\
Mantova (Italy), $\mathrm{n}=40$ & $22(55.0)$ & $15(37.5)$ & $3(7.5)$ \\
Maastricht (the Netherlands) ${ }^{24,} \mathrm{n}=25$ & $11(44.0)$ & $12(48.0)$ & $2(8.0)$ \\
Total study group, $\mathrm{n}=96$ & $52(54.2)$ & $34(35.4)$ & $10(10.4)$ \\
\hline
\end{tabular}

Results are expressed as absolute numbers of patients (percentage). CC denotes homozygosity for the C-encoded Thr1405 variant, AA homozygosity for the A-encoded Asn 1405 variant, and AC heterozygosity for this polymorphism.

As shown in table 4, when examining urea cycle intermediates in relation to the distribution of the CPS1 genotypes, we found no significant differences in arginine or citrulline concentrations. The levels of ornithine and the ornithine:citrulline ratio were also not significantly different among the three genotypes. Concentrations of other amino acids in plasma also showed no significant differences among the three genotypes (data not shown). Also no differences in ADMA and SMDA concentrations and arginine:ADMA ratio were observed among the CPS1 genotypes (table 4). 
Table 4. Urea cycle intermediates and methylated arginine per genotype

\begin{tabular}{lcccc}
\hline & $\begin{array}{c}\text { CC } \\
(\mathbf{n}=\mathbf{7 1})\end{array}$ & $\begin{array}{c}\text { CA } \\
(\mathbf{n = 4 3})\end{array}$ & $\begin{array}{c}\text { AA } \\
(\mathbf{n}=\mathbf{1 4})\end{array}$ & $\begin{array}{c}\boldsymbol{P} \\
\text { value }\end{array}$ \\
\hline Arginine & $41[24-60]$ & $40[19-57]$ & $38[28-60]$ & NS \\
Ornithine & $87[60-129]$ & $82[42-104]$ & $84[53-114]$ & \\
Citrulline & $21[17-25]$ & $20[15-26]$ & $23[16-26]$ & NS \\
Ornithine:Citrulline & $4.1[3.1-5.5]$ & $4.1[2.8-5.1]$ & $4.0[2.6-5.6]$ & NS \\
ADMA & $0.80[0.59-1.18]$ & $0.94[0.59-1.22]$ & $0.84[0.66-1.35]$ & NS \\
SMDA & $1.22[0.94-1.55]$ & $1.19[0.97-1.53]$ & $1.26[0.86-1.54]$ & NS \\
Arginine:ADMA & $50[11-70]$ & $40[26-56]$ & $47[31-74]$ & NS \\
\hline
\end{tabular}

Results of plasma concentrations ( $\mu \mathrm{mol} / \mathrm{I})$ are expressed as median [interquartile range]. Statistical analysis between the genotypes. NS = not significant . 


\section{DISCUSSION}

Identifying an early biomarker for infants at risk for NEC, i.e. preterm infants, remains an elusive research goal. Both, plasma L-arginine levels ${ }^{14,17,19-21}$ and the T1405N CPS1 polymorphim ${ }^{24}$ have been associated with the development of NEC. When Pearson et al. examined L-arginine and citrulline concentrations in relation to the distribution of the CPS1 genotypes, they found that term infants who were homozygous (CC) for the C-encoded Thr1405 enzyme had lower L-arginine concentrations than infants with the AA genotype and hence the Asn1405 enzyme. ${ }^{18}$ The present study was based on the hypothesis that in a population of preterm infants, in whom the urea cycle is not fully developed, genetically determined variations in CPS1 function would induce further changes in L-arginine levels. However, our data did not confirm this hypothesis because we did not find differences in urea cycle intermediates (i.e. citrulline and arginine) between the different CPS1 genotypes. This lack of effect of CPS1 genotype on L-arginine concentrations was also found in adults. ${ }^{22}$

$\mathrm{L}$-arginine concentrations as low as $3 \mu \mathrm{mol} / \mathrm{L}$ are sufficient to induce half-maximal activity of endothelial $\mathrm{NOS}^{28}$, whereas the neonatal L-arginine levels reported in the present and other studies is 10 - to 15 -fold higher. ${ }^{13,18,29}$ Clinical and experimental evidence indicates that elevation of the endogenous NOS inhibitor ADMA can cause a relative endothelial L-arginine deficiency, even in the presence of normal plasma Larginine levels. ${ }^{30}$ Elevated plasma levels of ADMA have been reported in diseases related to endothelial dysfunction including peripheral arterial disease, hypertension, hyperlipidemia, diabetes mellitus and hyerhomocysteinemia. ${ }^{15,31}$ However, the information on perinatal ADMA metabolism is limited. There are reports of elevated ADMA concentrations in umbilical venous plasma in neonates born term ${ }^{32}$ and elevated levels of ADMA have been involved in the pathogenesis of several neonatal conditions such as persistent pulmonary hypertension or infant respiratory distress syndrome. ${ }^{33,34}$ In a recent study, Richir et al. hypothesized that in addition to low arginine levels, infants with NEC might present increased ADMA plasma concentrations. ${ }^{14}$ Surprisingly, a significantly lower ADMA concentration was found in preterm infants with NEC. The levels of ADMA and SDMA and the ratio L- 
arginine:ADMA that we observed in our study are in the same range as previously reported in preterm infants ${ }^{14,34-36}$ and were not affected by the CPS1 genotype.

Although the present study was not powered to detect differences in amino acid levels or CPS1 genotype between infants with and without NEC, we observed that the mean L-arginine level of NEC infants showed a non-significant trend to lower values (median $29 \mu \mathrm{mol} / \mathrm{l}$, interquartile range 16-44 vs. median $40 \mu \mathrm{mol} / \mathrm{l}$, interquartile range 23-57 in non-NEC group). This trend was not present for citrulline, ADMA or SMDA levels. In addition, we observed that none of the five NEC patients presented the AA genotype. The C-encoded Thr1405 variant of CPS1 is considered the evolutionarily conserved version, whereas the less frequent, A-encoded Asn1405 variant appears to be a relatively new, gain-of-function mutation. ${ }^{18}$ Pearson et al. speculated that individuals with the AA genotype may have an advantage in terms of urea-cycle function and interrelated metabolic processes, especially under conditions of environmental stress. ${ }^{18}$ Most typically, NEC has its onset in VLBW infants after the second week of life. ${ }^{37}$ One limitation of our study is that we determined L-arginine levels between 6 and 12 hours after birth. It could be speculated that the alterations in arginine and citrulline levels related to genetic variations in CPS1 function may be only present later in life, under the NEC stress conditions, when the demand for NO might suddenly increase. However, it is also possible that the low arginine levels reported in NEC patients ${ }^{20,21}$ could be the consequence, rather than the cause of NEC. Serum citrulline was found in recent years to be a candidate marker of enterocyte mass and intestinal failure. ${ }^{38}$ Therefore, in the damaged intestine of NEC infants a lower release of citrulline into the bloodstream may lead to a lower rate of renal arginine production.

Maternal arginine levels may affect the delivery of arginine to the fetus and, consequently, to the infant. One of the limitations in this study is we did not measure amino acid levels in the mother and had no information about the maternal protein intake. Also, we did not measure any index of NO production, such as serum nitrite/nitrate. ${ }^{39}$ In addition, our study did not take into account the influence of other enzymes, such as pyrroline-5-carboxylate synthase, argininosuccinate synthase and argininosuccinate lyase, importantly involved in the intestinal arginine-synthetic pathway.$^{17}$ However, the activities of these enzymes were shown to be very low, at 
least in preterm piglets. ${ }^{17}$ Interestingly, it has been demonstrated that glucocorticoids stimulated the intestinal expression of pyrroline-5-carboxylate synthase, argininosuccinate synthase and argininosuccinate lyase in neonatal pig enterocytes. ${ }^{40-}$ ${ }^{42}$ However, we did not observe significant differences in arginine and citrulline levels between patients who received antenatal glucocorticoids (arginine median $40 \mu \mathrm{mol} / \mathrm{l}$, interquartile range 24-61; citrulline median $21 \mu \mathrm{mol} / \mathrm{l}$, interquartile range 17-24) and those who did not (arginine median $40 \mu \mathrm{mol} / \mathrm{l}$, interquartile range 21-57; citrulline median $24 \mu \mathrm{mol} / /$, interquartile range 16-30). Finally, our sample size calculation was based upon arginine levels in term newborns. ${ }^{18} \mathrm{It}$ is important to note that the mean arginine in our patients (AA genotype: $43.5 \mu \mathrm{mol} / \mathrm{L}, \mathrm{SD} 25.3$ ) was in the range of the one in Pearson's study (AA genotype: $35.7 \mu \mathrm{mol} / \mathrm{L}, \mathrm{SD}$ 7.6). However, the larger SD in our patients, reflecting the clinical heterogeneity of pretem infants, means that there might be a possibility of a Type II error underlying our negative results.

In conclusion, the earlier reported association between CPS1 genotype and L-arginine levels in term infants ${ }^{18}$, was not confirmed by the present study in preterm infants. Whether the T1404N CPS1 polymorphism is a good early biomarker for infants at risk for NEC, needs to be confirmed in a prospective cohort study, which we are conducting at this moment. 


\section{REFERENCES}

1 Lin, P.W., Nasr, T.R., Stoll, B.J. Necrotizing enterocolitis: recent scientific advances in pathophysiology and prevention. Semin Perinatol 32:70-82 (2008).

2 Schnabl, K.L., Van Aerde, J.E., Thomson, A.B., Clandinin, M.T. Necrotizing enterocolitis: a multifactorial disease with no cure. World $J$ Gastroenterol 14:2142-2161 (2008).

3 Obladen, M. Necrotizing Enterocolitis - 150 Years of Fruitless Search for the Cause. Neonatology 96:203-210 (2009).

4 Chauhan, M., Henderson, G., McGuire, W. Enteral feeding for very low birth weight infants: reducing the risk of necrotising enterocolitis. Arch Dis Child Fetal Neonatal Ed. 93:F162-166 (2008).

5 Rees, C.M., Eaton, S., Pierro, A. Trends in infant mortality from necrotising enterocolitis in England and Wales and the USA. Arch Dis Child Fetal Neonatal Ed. 93:F395-396 (2008).

6 Young, C., Sharma, R., Handfield, M., Mai, V., Neu, J. Biomarkers for Infants at Risk for Necrotizing Enterocolitis: Clues to Prevention? Pediatr Res. 65:91R97R (2009).

7 Henderson, G. et al. Cytokine gene polymorphisms in preterm infants with necrotising enterocolitis: genetic association study. Arch Dis Child Fetal Neonatal Ed. 94:F124-128 (2009).

8 Reber, K.M., Nankervis, C.A., Nowicki, P.T. Newborn intestinal circulation. Physiology and pathophysiology. Clin Perinatol 29:23-39 (2002).

9 Upperman, J.S. et al. Mechanisms of nitric oxide-mediated intestinal barrier failure in necrotizing enterocolitis. Semin Pediatr Surg 14:159-166 (2005).

10 Chokshi, N.K. et al. The role of nitric oxide in intestinal epithelial injury and restitution in neonatal necrotizing enterocolitis. Semin Perinatol 32:92-99 (2008).

11 Nowicki, P.T. et al. Intestinal O2 consumption in necrotizing enterocolitis: role of nitric oxide. Pediatr Res 59:500-505 (2006).

12 Anand, R.J., Leaphart, C.L., Mollen, K.P., Hackam, D.J. The role of the intestinal barrier in the pathogenesis of necrotizing enterocolitis. Shock 27:124-133 (2007). 
13 Vida, G., Sulyok, E., Ertl, T., Martens-Lobenhoffer ,J., Bode-Boger, S.M. Plasma asymmetric dimethylarginine concentration during the perinatal period. Neonatology 92:8-13 (2007).

14 Richir, M.C. et al. Low plasma concentrations of arginine and asymmetric dimethylarginine in premature infants with necrotizing enterocolitis. $\mathrm{Br} J$ Nutr 97:906-911 (2007).

15 De Gennaro Colonna, V. et al. Asymmetric dimethylarginine (ADMA): an endogenous inhibitor of nitric oxide synthase and a novel cardiovascular risk molecule. Med Sci Monit 15:RA91-101 (2009).

16 Wu, G. Amino acids: metabolism, functions, and nutrition. Amino Acids 37:1-17 (2009).

17 Wu, G., Jaeger, L.A., Bazer, F.W., Rhoads, J.M. Arginine deficiency in preterm infants: biochemical mechanisms and nutritional implications. J Nutr Biochem 15:442-451 (2004).

18 Pearson, D.L. et al. Neonatal pulmonary hypertension--urea-cycle intermediates, nitric oxide production, and carbamoyl-phosphate synthetase function. N Engl J Med 344:1832-1838 (2001).

19 Amin, H.J. et al. Arginine supplementation prevents necrotizing enterocolitis in the premature infant. J Pediatr 140:425-431 (2002).

20 Becker, R.M. et al. Reduced serum amino acid concentrations in infants with necrotizing enterocolitis. J Pediatr 137:785-793 (2000).

21 Zamora, S.A. et al. Plasma L-arginine concentrations in premature infants with necrotizing enterocolitis. J Pediatr 131:226-232 (1997).

22 Summar, M.L. et al. Relationship between carbamoyl-phosphate synthetase genotype and systemic vascular function. Hypertension 43:186-191 (2004).

23 Kallianpur, A.R. et al. The hemochromatosis C282Y allele: a risk factor for hepatic veno-occlusive disease after hematopoietic stem cell transplantation. Bone Marrow Transplant 35:1155-1164 (2005).

24 Moonen, R.M. et al. Carbamoyl phosphate synthetase polymorphisms as a risk factor for necrotizing enterocolitis. Pediatr Res 62:188-190 (2007).

25 van Eijk, H.M., Rooyakkers, D.R., Deutz, N.E. Rapid routine determination of amino acids in plasma by high-performance liquid chromatography with a 2-3 microns Spherisorb ODS II column. J Chromatogr 620:143-148 (1993). 
26 Waterval, W.A., Scheijen, J.L., Ortmans-Ploemen, M.M., Habets-van der Poel, C.D., Bierau, J. Quantitative UPLC-MS/MS analysis of underivatised amino acids in body fluids is a reliable tool for the diagnosis and follow-up of patients with inborn errors of metabolism. Clin Chim Acta 407:36-42 (2009).

27 Martens-Lobenhoffer, J., Bode-Boger, S.M. Chromatographic-mass spectrometric methods for the quantification of $\mathrm{L}$-arginine and its methylated metabolites in biological fluids. J Chromatogr B Analyt Technol Biomed Life Sci 851:30-41 (2007).

28 Pollock, J.S. et al. Purification and characterization of particulate endotheliumderived relaxing factor synthase from cultured and native bovine aortic endothelial cells. Proc Natl Acad Sci U S A 88:10480-10484 (1991).

29 van den Berg, A. et al. A randomized controlled trial of enteral glutamine supplementation in very low birth weight infants: plasma amino acid concentrations. J Pediatr Gastroenterol Nutr 41:66-71 (2005).

30 Boger, R.H. Asymmetric dimethylarginine, an endogenous inhibitor of nitric oxide synthase, explains the "L-arginine paradox" and acts as a novel cardiovascular risk factor. J Nutr 134:2842S-2847S (2004).

31 Siroen, M.P. et al. The clinical significance of asymmetric dimethylarginine. Annu Rev Nutr 26:203-228 (2006).

32 Maeda, T., Yoshimura, T., Okamura, H. Asymmetric dimethylarginine, an endogenous inhibitor of nitric oxide synthase, in maternal and fetal circulation. J Soc Gynecol Investig 10:2-4 (2003).

33 Pierce, C.M., Krywawych, S., Petros, A.J. Asymmetric dimethyl arginine and symmetric dimethyl arginine levels in infants with persistent pulmonary hypertension of the newborn. Pediatr Crit Care Med 5:517-520 (2004).

34 Richir, M.C. et al. Plasma ADMA concentrations at birth and mechanical ventilation in preterm infants: a prospective pilot study. Pediatr Pulmonol 43:1161-1166 (2008).

35 Vida, G. et al. Plasma levels of asymmetric dimethylarginine in premature neonates: its possible involvement in developmental programming of chronic diseases. Acta Paediatr 98:437-441 (2009).

36 Mittermayer, F., Prusa, A.R., Pollak, A., Wolzt, M. Umbilical vein plasma concentrations of asymmetrical dimethylarginine are increased in male but not 
female neonates delivered preterm: a pilot study. Early Hum Dev 82:421-424 (2006).

37 Gordon, P.V., Swanson, J.R., Attridge J.T., Clark, R. Emerging trends in acquired neonatal intestinal disease: is it time to abandon Bell's criteria? $J$ Perinatol 27:661-671 (2007).

38 Crenn, P., Coudray-Lucas, C., Thuillier, F., Cynober, L., Messing, B. Postabsorptive plasma citrulline concentration is a marker of enterocyte mass and intestinal failure in humans. Gastroenterology 119:1496-1505 (2000).

39 Figueras-Aloy, J. et al. Plasma nitrite/nitrate and endothelin-1 concentrations in neonatal sepsis. Acta Paediatr 92:582-587 (2003).

40 Flynn, N.E., Wu, G. Enhanced metabolism of arginine and glutamine in enterocytes of cortisol-treated pigs. Am J Physiol 272:G474-G480 (1997).

41 Flynn, N.E., Wu, G. Glucocorticoids play an important role in mediating the enhanced metabolism of arginine and glutamine in enterocytes of postweaning pigs. J Nutr 127:732-737 (1997).

42 Flynn, N.E. et al. Glucocorticoids mediate the enhanced expression of intestinal type II arginase and argininosuccinate lyase in postweaning pigs. J Nutr 129:799-803 (1999).

Acknowledgments: Supported by grants from: 1) Fundacion de Investigacion Medica Mutua Madrilena (http://www.mutua-mad.es/FundMM/jsp/Fhome.jsp), 2) Sociedad Espanola de Neonatologi'a, (http://www.se-neonatal.es). The funders had no role in study design, data collection and analysis, decision to publish, or preparation of the manuscript.

We thank the investigators of the department of Surgery, University of Maastricht, and department of Clinical Genetics, Maastricht University Medical Center, for providing amino acid and polymorphisms analysis. 
Chapter 8

SUMMARY, GENERAL DISCUSSION AND FUTURE PERSPECTIVES

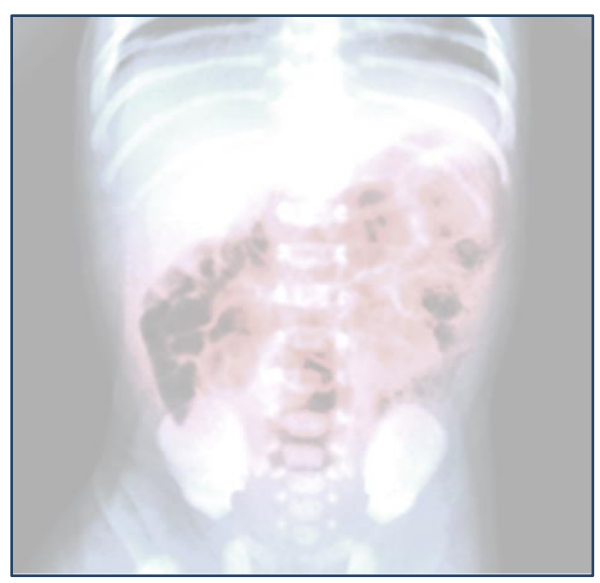


At present necrotizing enterocolitis (NEC) is still a leading cause of morbidity and mortality in very low birthweight (VLBW) infants. The pathogenesis of NEC remains elusive and is most likely multifactorial. One of the suspected pathophysiological mechanisms is the developmental immaturity of circulatory regulation. ${ }^{1-8}$ However, our current understanding of the fetal and neonatal intestinal vascular physiology and pathophysiology is limited.

The present thesis focused on the role of the vascular system in the pathogenesis of NEC and approached the problem from two perspectives. In the first part, we investigated the developmental changes in mesenteric arterial reactivity under physiological and pathological (i.e. hypoxic) conditions. In the second part, we analyzed the role of L-arginine, the precursor for the synthesis of nitric oxide (NO), on the pathogenesis of NEC. This chapter will provide a general discussion on the two topics of the thesis.

\section{PART I DEVELOPMENTAL CHANGES IN MESENTERIC ARTERIAL REACTIVITY UNDER PHYSIOLOGICAL AND PATHOLOGICAL CONDITIONS}

\section{NORMAL DEVELOPMENT}

A balance between a vasodilator (NO) and a vasoconstrictor (ET-1) appears to be the main factor controlling intestinal vascular resistance in the mammalian fetus and newborn. It has been proposed that a misbalance between these two vasoactive mediators may predispose the immature intestine to NEC. ${ }^{9}$

$\mathrm{NO}$ is endogenously synthesized through the action of the NO synthase (NOS) family of enzymes. Three NOS isoforms have been identified sharing a $50-60 \%$ homology; two constitutive, the neuronal (nNOS, type I) and endothelial (eNOS, type III) enzymes, and one inducible (iNOS, type II). ${ }^{10,11}$ Now it is appreciated that eNOS is found in other cells and tissues besides the endothelium, iNOS is found constitutively in some tissues, and there are inducible forms of both eNOS and nNOS. ${ }^{12}$ The NO-generating reaction requires L-arginine, NADPH and oxygen as substrates, and tetrahydrobiopterin (BH4), thiol, flavin adenine dinucleotide, and flavin mononucleotide 
as cofactors. In addition to NO, the NOS-catalyzed reaction produces citrulline and NADP as co-products.

ET-1 is a vasoactive and mitogenic polipeptide produced mainly by the vascular endothelium. Its binding to $\mathrm{ET}_{\mathrm{A}}$ and $\mathrm{ET}_{\mathrm{B}}$ receptors on vascular smooth muscle induces contraction, whereas its binding to endothelial $\mathrm{ET}_{\mathrm{B}}$ receptors causes vasodilation. ${ }^{13,14}$ ET-1 is constitutively produced, but its production can also be stimulated by a wide range of stimuli, including decreased flow, hypoxia, and various inflammatory cytokines. ${ }^{14,15}$

In the mammalian newborn, the balance NO/ET-1 favors vasodilation generating an increased rate of blood flow to the intestine to meet the metabolic demands of early postnatal life. Our data suggest the presence of similar regulatory mechanisms in the chicken mesenteric circulation (chapter 2). Moreover, maturation of vasodilator mechanisms precedes that of vasoconstrictor mechanisms. As early as 0.7 incubation, the chicken embryo mesenteric arteries show a high capacity to relax in response to the NO-cGMP pathway as well as to the adenylate cyclase-cAMP pathway and to a putative endothelium derived hyperpolarizing factor (EDHF). This ability to relax of the fetal arteries is maintained during the first days post-hatching. In contrast, ET-1mediated contraction of chicken embryo mesenteric arteries was weak in the less mature embryos, peaked at 0.9 incubation, and decreased during late incubation and the post-hatching period. Moreover, high concentrations of ET-1 induced relaxation in the mesenteric arteries from newly hatched chicks. This suggests the presence of

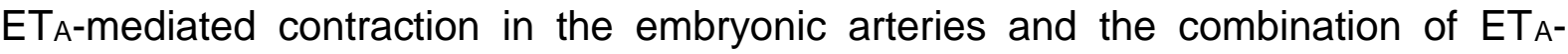
mediated contraction and $\mathrm{ET}_{\mathrm{B}}$-mediated relaxation in the mesenteric arteries from the newly hatched chicks. When other vasoconstrictors such as norepinephrine or thromboxane A2 were tested, it was observed that their vasoactive efficacy increased in ovo but not ex ovo.

Very recently, Nair et al. ${ }^{16}$ investigated the ex vivo reactivity of mesenteric arteries from fetal (116-d, 128-d, 134-d, and 141-d gestation, term $147 \mathrm{~d}$ ) and postnatal (2- to 7$d$-old) lambs and observed important similarities with our study in chicken embryos. At early gestation (116-d and 128-d), mesenteric arteries constrict poorly to NE, and ET- 
1, but NO-mediated relaxation appear to be functional. At late preterm gestation (134$d$ and 141-d), they observed a significant increase in contractility to ET-1 and NE together with a developmental increase in NO-mediated, endothelium-dependent relaxation. Interestingly, this increase was not accompanied by changes in basal NO production, which even decreased in the 134-d animals. Finally, during the postnatal period, the balance tips in favor of vasorelaxation. Contraction to NE and ET-1 were markedly diminished, basal NOS activity was high, and endothelium-dependent as well as -independent relaxation were markedly increased. ${ }^{16}$

Taking together our results (chapter 2) and the results of Nair et $\mathrm{al}^{16}$ as well as our previous investigation in fetal lamb mesenteric arteries ${ }^{17}$, vessels from late gestation animals ( 0.9 incubation chickens and 0.9 gestation lambs) show the highest imbalance between mediators of constriction and relaxation favoring the former. During the postnatal period, after introduction of feeds, the balance shifts in favor of vasorelaxation. These changes may enable the mesenteric circulation to handle postprandial hyperemia required to digest and absorb nutrients following intermittent feeds. ${ }^{16}$ Very immature mesenteric arteries ( 0.7 incubation chickens and 0.8 gestation lambs) show a predominant vasodilator tone with very weak responses to vasoconstrictor agonists. As previously postulated by Nankervis et al ${ }^{9}$ interventions focused on maintaining the delicate balance favoring vasodilation in the newborn intestinal circulation may prove to be useful in the prevention and treatment of perinatal intestinal diseases such as NEC.

\section{EFFECTS OF PRENATAL CHRONIC HYPOXIA ON INTESTINAL DEVELOPMENT}

Prenatal development takes place in an environment that is relatively hypoxic compared with the postnatal environment. ${ }^{15,18}$ Besides this physiological hypoxia, the fetus can be exposed to acute, intermittent, or chronic hypoxic challenges, which can lead to injury or death. ${ }^{18-21}$ Acute or chronic hypoxic injury to the gastrointestinal tract is believed to be a major contributing and potentially inciting factor in several neonatal conditions, including NEC. The chicken embryo is a valuable model for the study of the pathophysiological effects of acute and chronic prenatal hypoxia. ${ }^{18,22-35}$ Hypoxia is easily induced by incubating the egg in a low oxygen environment and its effects can 
be studied without interferences of maternal hormonal, metabolic, or hemodynamic alterations.

In chapter 3 we focused on how mesenteric vascular reactivity and intestinal development of chicken embryos and hatchlings was affected by chronic moderate hypoxia (15\% O2) during incubation. Hypoxic incubation increased the responsiveness of chicken embryo mesenteric arteries to norepinephrine and impaired both endothelium-dependent and -independent relaxation. However, all these alterations were transient and, therefore, were not present in the newly hatched chicks. In contrast, hypoxic incubation induced hyperresponsiveness to ET-1 and this increased contraction persisted during the first hours of postnatal life. As discussed above, the prenatal mesenteric circulation is characterized by a high vasoconstrictive tone mediated by catecholamines and ET-1 (chapter 2 and Nair et $\mathrm{al}^{16}$ ) but this tone shifts to vasodilation in the first days of postnatal life. A decreased contraction to ET-1 is, at least partially, responsible for the reduced tone of neonatal mesenteric arteries (chapter 2 and Nair et $\mathrm{al}^{16}$ ). Our data suggest that chronic hypoxia may impair this transition through the maintenance of a high sensitivity to ET-1-mediated contraction. Moreover, our results in the chicken embryo (chapter 3) indicate that ET-1-induced relaxation is impaired by incubation under chronic hypoxia, unmasking a higher ET-1induced contraction. It can be speculated that this exacerbated vasoconstriction might impair the increase in intestinal blood flow that accompanies intermittent enteral feedings in the newborn. Interestingly, ET-1 concentration is greater in human preterm intestine that demonstrates histologic evidence of NEC, and arterioles taken from these tissue samples showed increased vasoconstriction which reversed following addition of the ETA antagonist BQ610. ${ }^{36}$ Therefore, as suggested by Nair et al., ET-1 blocking agents/antagonists may represent an additional candidate for novel therapy for NEC. ${ }^{16}$

We also analysed the effects of chronic hypoxia on intestinal development and on intestinal expression of VEGF. In accordance with other authors ${ }^{37-41}$, we observed a progressive but slow increase in villus development between days 15 and 19 of incubation and rapid morphological changes after hatch (chapter 3). Chronic exposure to $15 \% \mathrm{O}_{2}$ induced only subtle alterations on this pattern of development. In fact, in 
ovo intestinal development was similar in normoxic and hypoxic embryos. After hatching, the hypoxic chicks showed decreased villus length and muscularis propria thickness but this slight underdevelopment was not more present in 1-d-old chicks. In addition, we found that intestinal VEGF expression increased toward hatching and that this increase was impaired by hypoxic incubation. Similarly, developmental increases in VEGF have been demonstrated in lamb intestine ${ }^{42}$, suggesting a role for VEGF in the development of the intestinal vascular network during fetal and early postnatal life. Although hypoxia is regarded as the most potent regulator of VEGF ${ }^{43}$, we did not find a higher VEGF expression in the intestine from the hypoxic embryos. However the hypoxic embryos did not undergo the posthatch increase in VEGF expression observed in the normoxic embryos. It can be speculated that the rapid intestinal growth that takes place between 19 and 21 days of incubation induces relative tissular hypoxia leading to increased VEGF expression. Embryos incubated in low $\mathrm{O}_{2}$ increase carrying capacity through increases in red blood cells, hemoglobin mass, or blood volume. ${ }^{44}$ Such adaptations to low $\mathrm{O}_{2}$ may have reduced the physiological hypoxia induced by rapid intestinal growth and, consequently, the intestinal expression of VEGF.

In summary the series of experiments reported in chapter $\mathbf{3}$ present evidence that chronic moderate hypoxia during incubation results in subtle but significant alterations in chicken MA reactivity, small intestine morphology and VEGF expression. Whether these slight alterations may have a direct effect on the functional status of chick intestine remains uncertain. It can be speculated that the modest degree of the alterations induced by hypoxia is the result of the adaption of the embryo to the prolonged exposure to a relatively moderate insult.

\section{ACUTE HYPOXIA}

Either in the mammalian fetus or the chicken embryo the cardiovascular responses to acute hypoxia includes a redistribution of the cardiac output away from the periphery towards high priority organs such as the heart, brain, and adrenal glands. ${ }^{21,45-47} \mathrm{An}$ important component of hypoxia-induced flow redistribution is mediated by increased release of catecholamines ${ }^{48-51}$, whose effects are modulated by the different responsiveness of the vascular beds. However, hypoxia-mediated redistribution of blood flow is also accomplished by a local vascular effect, which results in contraction 
or relaxation depending on the blood vessel. It is generally accepted that pulmonary arteries respond to regional hypoxia with vasoconstriction (hypoxic pulmonary vasoconstriction, HPV), whereas systemic arteries dilate..$^{52-55}$

In chapter $\mathbf{4}$ we demonstrated that hypoxic vasoconstriction is an intrinsic feature of chicken mesenteric arteries during late embryogenesis and the perinatal period. Agonist-induced pretone was not required to obtain a consistent and reproducible response, which showed a pattern of initial rapid phasic contraction followed by a sustained tonic contraction. The two phases demonstrated different properties. The phasic contraction was reduced by the elimination of extracellular $\mathrm{Ca}^{2+}$ or by the presence of the neurotoxin tetrodotoxin, the $\alpha_{1}$-adrenoceptor antagonist prazosin, or inhibitors of L-type voltage-operated $\mathrm{Ca} 2+$ channels, the mitochondrial electron transport chain (mETC), and NADPH oxidase. The tonic contraction was markedly impaired by the presence of the SERCA inhibitor thapsigargin. Rho-kinase inhibition impaired both phasic and tonic contraction and, when combined with elimination of extracellular $\mathrm{Ca}^{2+}$, hypoxia-induced contraction was virtually abolished.

Regarding developmental changes, we observed that hypoxia-induced contraction in isolated mesenteric arteries from chickens was absent in the more immature embryos (i.e., 15-d) but present in the late embryonic period (i.e., 19-d). The response increased towards the first days post hatching, after which it decreased during the first weeks of life. Interestingly, the decrease with age in hypoxic mesenteric contraction resulted in a lack of ability of mesenteric arteries from 45 -d-old chickens to sustain prolonged contraction. Therefore, our data suggest that hypoxia-induced contraction of isolated mesenteric arteries is a distinctive feature of the late fetal and perinatal period.

Although it is clear that intestinal ischemia plays a significant role in the pathogenesis of NEC, it is controversial whether this role is primary or secondary and what mechanisms lead to the ischemia. ${ }^{56,57}$ Epidemiologic evidence suggests that the most common form of NEC in very preterm infants is not triggered by a primary hypoxicischemic event. ${ }^{57}$ Moreover, the near-midgestation fetus shows attenuated vasomotor responses to severe asphyxia compared with more mature fetuses. ${ }^{57}$ Our results showing that catecholamine-induced contraction (chapter 2) and hypoxic mesenteric 
vasoconstriction (chapter 4) were poorly developed in the less mature mesenteric arteries, indirectly support the hypothesis that the immature intestine is less susceptible to direct hypoxic damage. Conversely, the capacity of the cardiovascular system of very preterm fetuses to redistribute blood flow output away from the intestinal circulation in response to acute hypoxia might be diminished, making less mature fetuses more susceptible to hypoxic-ischemic brain damage. 


\section{PART II: THE L-ARGININE/NO PATHWAY AND THE RISK OF NEC}

NO has important functions in a number of gastrointestinal physiologic and pathophysiologic processes, including motility, mucosal function, blood regulation and inflammatory responses. ${ }^{58}$ While the influence of NO on gastrointestinal motility is largely controlled by neural NO-synthase (nNOS), vascular effects occur largely under the purview of endothelial NO-synthase (eNOS). Additionally, inducible NO-synthase (iNOS)-derived NO production contributes to inflammatory processes. The substrate for eNOS, nNOS, and iNOS is the amino acid L-arginine. ${ }^{11,59} \mathrm{~L}$-arginine is an intermediate product of the urea cycle (Fig. 1), the major route to excrete waste nitrogen in humans. ${ }^{12,60}$

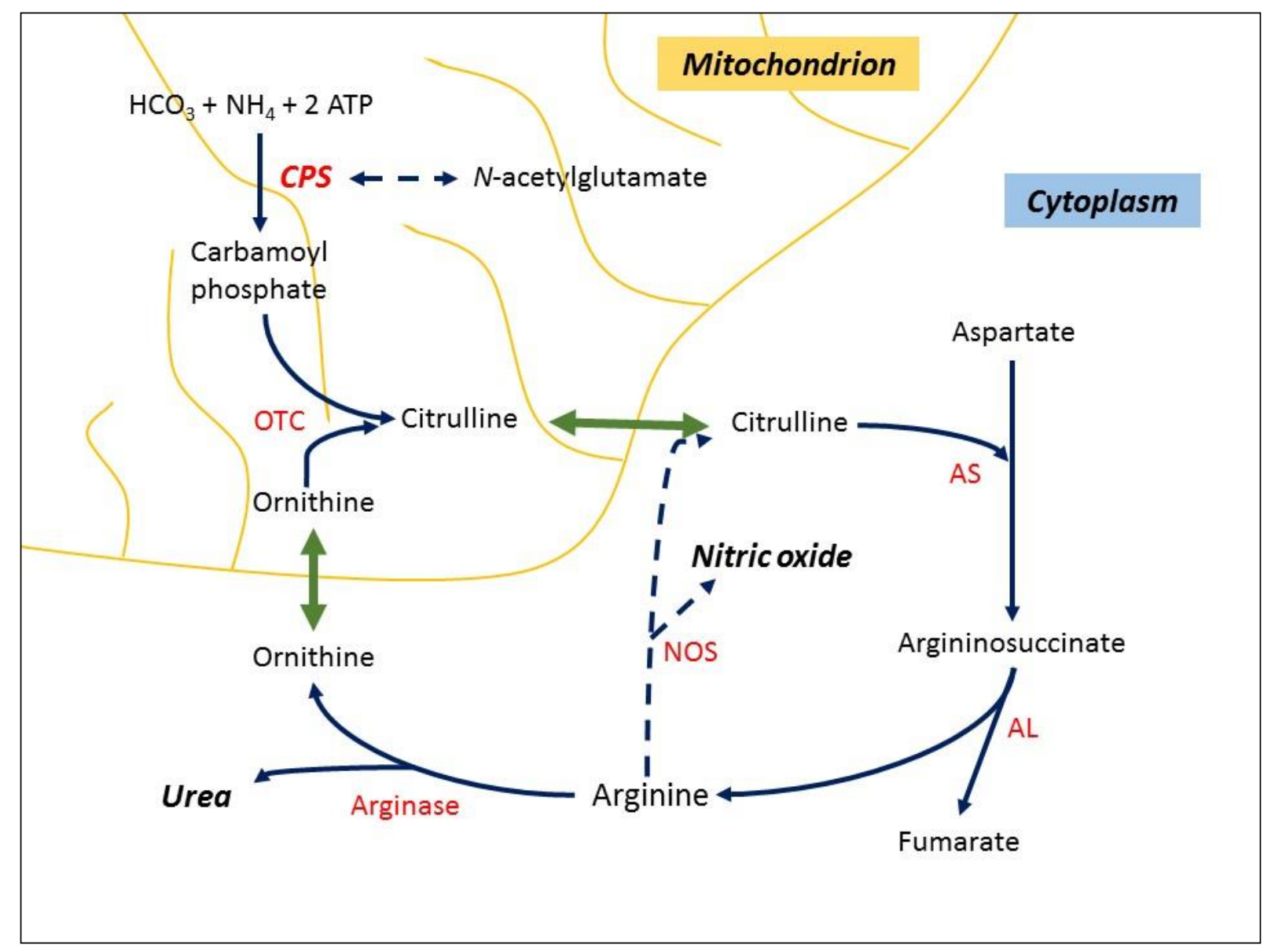

Figure 1. Enzymes and Intermediates in the Urea Cycle and Nitric Oxide Pathway. The mitochondrial enzymes are present only in the urea cycle in the liver. The cytoplasmic enzymes argininosuccinate synthase (AS) and argininosuccinate lyase (AL) are also present in endothelial cells, where they recycle citrulline into arginine for high-output nitric oxide synthesis. CPS denotes carbamoyl-phosphate synthetase, NOS nitric oxide synthase, and OTC ornithine transcarbamylase. 
Carbamoyl phosphate synthetase 1 (CPS1, OMIM 608307) is the rate-limiting enzyme catalysing the first committed step of the urea cycle to produce carbamoyl phosphate. The human CPS1 gene is located on chromosome 2q34-35 and expands more than $120 \mathrm{~kb}$, encompassing 38 exons and 37 introns. It encodes a polypeptide of 1500 amino acids. Human CPS1 is mainly expressed in liver and intestine cells, and at low levels in kidney tissue and fibroblasts. ${ }^{60} \mathrm{~A}$ three-step irreversible reaction catalyzed by CPS1 (2ATP $+\mathrm{HCO}_{3}^{-}+\mathrm{NH}_{3} \rightarrow 2 \mathrm{ADP}+\mathrm{Pi}+$ carbamoyl-phosphate) yields the carbamoyl-phosphate used to make the arginine precursor citrulline. CPS1 uses ammonia with high affinity and cannot use glutamine, and it requires $\mathrm{N}$-acetyl-Lglutamate (NAG), a sensor of excess amino acids that yield excess ammonia upon degradation. NAG is an allosteric activator without which CPS1 is inactive. ${ }^{60}$

CPS1 deficiency results in life-threatening hyperammonemia and has been reported in patients of all ages. Patients with early-onset CPS1 deficiency present in the first days of life with acute metabolic decompensation. Patients who survive the neonatal period often have periodic episodes of hyperammonemia due to viral infections or increased dietary protein intake. Patients with late-onset disease demonstrate a more heterogeneous clinical picture such as feeding difficulties, failure to thrive, psychomotor retardation, and late-onset acute metabolic decompensation with hyperammonemia. ${ }^{60}$

Several mutations over the entire human CPS1 sequence have been described in patients with CPS1 deficiency. CPS1 deficiency can also be due to the lack of the essential cofactor NAG. ${ }^{61}$ In addition, several non-pathological and pathological natural genetic variants (single nucleotide polymorphisms, SNPs) of CPS1 have been reported in the human population. ${ }^{60,62}$ One of those SNPs (p.Thr1406Asn also published as $\mathrm{T} 1405 \mathrm{~N}$; rs 1047891 formerly designated as rs7422339) has been linked to functional consequences affecting the downstream availability of urea-cycle intermediates, including L-arginine. ${ }^{62,63}$ The SNP p.Thr1406Asn is a C-to-A nucleotide transversion (c.4217C $>A$ ) in exon 36 , which results in the substitution of asparagine (Asn) for threonine (Thr) in the critical $\mathrm{N}$-acetylglutamate-binding domain. ${ }^{60}$ The $\mathrm{C}$ encoded Thr form of the polymorphism is considered the evolutionarily conserved version and the less frequent, A-encoded, Asn variant appears to be a relatively new, 
gain-of-function mutation. ${ }^{64}$ It has been suggested that the A-allele may confer an advantage in terms of NO production, especially under conditions of environmental stress. ${ }^{65}$ Previous studies demonstrated the association of the CPS1 p.Thr1406Asn genotype with clinical situations where endogenous NO production is critically important, such as persistent pulmonary hypertension of the newborn, pulmonary hypertension following surgical repair of congenital heart defects, or hepatic venoocclusive disease following bone marrow transplantation. ${ }^{64-66}$

L-arginine is an essential amino acid for young mammals. ${ }^{67,68}$ Metabolic and molecular studies indicate that the underdevelopment of intestinal arginine synthesis may be primarily responsible for remarkably low plasma arginine concentrations in preterm neonates. ${ }^{65,69}$ Several studies demonstrated that plasma arginine concentrations are even more decreased in premature infants with NEC.68,70-73 Moreover, arginine supplementation reduced the incidence of NEC in two small randomized controlled studies. ${ }^{70,74,75}$ Not only plasma arginine concentrations but also plasma citrulline concentrations are decreased in premature infants with NEC. ${ }^{72,76}$ Nevertheless, the mechanisms explaining the relative deficiency of L-arginine and L-citrulline in infants with NEC are far from being understood.

We hypothesized that variations in the p.Thr1406Asn genotype are associated with the risk of developing NEC. This hypothesis was initially tested in a retrospective casecontrol study (chapter 5) and proved to be true. In order to confirm the results of the retrospective study, we conducted a multicenter prospective cohort study in which the association between the p.Thr1406Asn genotype and NEC risk (chapter 6) and Larginine levels (chapter 7 ) were investigated.

\section{CARBAMOYL-PHOSPHATE SYNTHETASE POLYMORPHISMS AS A RISK FACTOR FOR NECROTIZING ENTEROCOLITIS: A CASE CONTROL-STUDY.}

We hypothesized that variations in the p.Thr1406Asn genotype are associated with the risk of developing NEC. To test our hypothesis we conducted a case-control study (chapter 5). In this retrospective, relatively small case-control study we found that patients with NEC showed an overrepresentation of the C-encoded variant of CPS1 
( $82,4 \%$ in NEC infants vs. $58.8 \%$ in the preterm control group, $p=0,018)$. Linear trend analysis demonstrated the quantitative increase of $C$ allele in NEC and homozygosity for $C$ allele increased the risk of having NEC ( $p=0.037)$. The CC genotype was associated with an increased risk of NEC in the preterm infants (odds ratio (OR) 3.43, 95\% confidence interval $(\mathrm{Cl}) 1.01-11.49, p=0.048)$, when compared with the grouped together AA/AC genotypes. These data suggest that the $\mathrm{p}$.Thr1406Asn polymorphism of CPS1 genotype may be associated with the risk of NEC in preterm infants. Although the case and the controls were well matched and there was no significant association of gestational age $(p=0.952)$ or birth weight $(p=0.650)$ with NEC in the logistic regression model, when adjusted for these two known risk factors for NEC, the OR did not remain significant (adjusted OR 3.246, 95\% Cl 0.91-11.52, $p=0.069$ ). Therefore, whether the p.Thr1406Asn genotype is an independent risk factor for NEC needed to be confirmed in a study involving a larger population.

\section{ASSOCIATION BETWEEN THE PTHR1406ASN POLYMORPHISM OF THE CARBAMOYL-PHOSPHATE SYNTHASE 1 GENE AND NECROTIZING ENTEROCOLITIS: A PROSPECTIVE MULTICENTER STUDY.}

In chapter 6 we described a large $(n=477)$ prospective cohort study. Allele and genotype frequencies of the p.Thr1406Asn polymorphism did not significantly differ between the infants with and without NEC. In contrast, the minor A-allele was significantly less frequent in the group of 64 infants with the combined outcome NEC or death before 34 weeks of corrected gestational than in the infants without the outcome ( 0.200 vs. $0.311, P=0.03)$. In addition, a significant negative association of the A-allele with the combined outcome NEC or death was found using the dominant (adjusted OR 0.54, 95\% Cl 0.29-0.99, $P=0.04$ ) and the additive (adjusted OR 0.58, $95 \% \mathrm{Cl} 0.36-0.93, P=0.03)$ genetic models. Thus, in other words, the minor $\mathrm{A}$ variant of the polymorphism might protect against this combined outcome. 


\section{THE PTHR1406ASN POL YMORPHISM OF THE CARBAMOYL-PHOSPHATE SYNTHASE 1 DOES NOT AFFECT PLASMA ARGININE CONCENTRATIONS IN PRETERM INFANTS.}

Pearson et al reported an association between CPS1 genotype and L-arginine levels in term infants. ${ }^{65}$ Chapter 7 was based on the hypothesis that in a population of preterm infants, in whom the urea cycle is not fully developed, genetically determined variations in CPS1 function would induce further changes in L-arginine levels. However, our data did not confirm this hypothesis because we did not find differences in urea cycle intermediates (i.e. citrulline and arginine) between the different CPS1 genotypes. There was no association between the CPS1 genotype and plasma Larginine or L-citrulline concentration, or the ornithine to citrulline ratio, which varies inversely with CPS1 activity. One limitation of our study is that we determined Larginine levels between 6 and 12 hours after birth. It could be speculated that the alterations in arginine and citrulline levels related to genetic variations in CPS1 function may be only present later in life, under the NEC stress conditions, when the demand for NO might suddenly increase. However, it is also possible that the low arginine levels reported in NEC patients ${ }^{65,68,70,71,73,74}$ could be the consequence, rather than the cause of NEC

In summary, our results from chapters 5,6 , and 7 suggest that functional genetic variations in the CPS enzyme might be, at least partially, the link between hypoarginimemia and NEC in preterm infants. $68,70-72,74$ Although at birth the levels of urea cycle intermediates are similar among the different CPS1 genotypes, the minor A-allele of the pThr1406Asn polymorphism of CPS1, appears to confer protection against the combined outcome of NEC/death. It should be noted that in situations of reduced availability of the substrate $\mathrm{L}$-arginine or the cofactor $\mathrm{BH}_{4}$, NOS enzymatic activity becomes uncoupled, resulting in the production of superoxide instead of NO. ${ }^{73}$ Therefore, besides resulting in a paucity of NO, the uncoupled enzyme will generate free radicals resulting in further intestinal damage. In addition, besides NO, arginine is a substrate for synthesis of many biologically important molecules including agmatine, polyamines, and creatine. ${ }^{68,77,78}$ These metabolites have roles in energy metabolism, gene expression, apoptosis, and cell proliferation and differentiation, which are crucial in intestinal homeostasis. $68,78,7$ 


\section{FUTURE PERSPECTIVES}

\section{EFFECTS OF PRE- AND PERINATAL HYPOXIC INSULTS ON MESENTERIC VASCULAR HOMEOSTASIS}

Understanding the basic mechanisms of either normal or altered functional and structural development of the mesenteric vessels, as well as inter-species differences in mesenteric circulation, may provide insights into human intestinal disease. Critical time windows exist during development, and if environmental changes, such as acute or chronic hypoxia, are experienced in the window of vulnerability, then the trajectory of development of the responding organ may be changed in ways that result in transient or persistent alterations..$^{80}$ In chapter 3 of this thesis, we characterized the effects of chronic in ovo hypoxia on mesenteric vascular reactivity but our study was limited to the first days of postnatal life. Future studies will be focused on analyzing whether the vascular alteration induced by chronic hypoxia persist during adult life. In chapter 4, we characterized the developmental changes in hypoxic mesenteric vasoconstriction in the chicken embryo. Either in human newborns or in chicken embryos pre- and perinatal to hypoxia was related to an enhancement of hypoxic pulmonary vasoconstriction in adult life. ${ }^{81,82}$ Future studies will be focused on investigating whether hypoxic mesenteric vasoconstriction is affected by chronic prenatal hypoxia.

One of the most common challenges to the fetus during gestation is an acute reduction in its oxygenation. Reoxygenation following exposure to acute hypoxia may increase the hypoxic damage. ${ }^{83-86}$ In experimental and clinical studies, it has been shown that the use of room air ( $21 \%$ oxygen) offers substantial advantages over the use of $100 \%$ oxygen, as had been traditionally recommended in the resuscitation of depressed newborn infants. ${ }^{83}$ Production of reactive oxygen species (ROS) is a key component of the pathogenesis of hypoxia-reoxygenation injury. ${ }^{86,87}$ Vascular endothelial cells are the primary target for ROS generated by themselves immediately after reoxygenation/reperfusion and, at later times, by adherent leukocytes. ${ }^{87,88}$ In addition, ROS-mediated injury of vascular smooth muscle cells may amplify the damage. 88,89 Our group has recently characterized a model of acute hypoxia-reoxygenation in the chicken embryo (Van Zogchel et al. unpublished). Exposure of 19-d embryos to 10\% 
$\mathrm{O}_{2}$ for $30 \mathrm{~min}$ induced an 8-fold increase in blood lactate. Our laboratory is currently investigating the effects of this sub-lethal hypoxic exposure, followed by normoxic or hyperoxic reoxygenation, on mesenteric vascular reactivity. We hypothesized that hypoxia-reoxygenation affect particularly endothelium-dependent relaxation and hypoxic mesenteric vasoconstriction. In addition the intestinal damage induced by hypoxia-reoxygenation will be investigated.

\section{PROSPECTIVE GENETIC BIOMARKERS FOR NEC RISK}

NEC affects only a minority of preterm infants, which suggest an individual susceptibility toward the disease. Analyses of twin gestations support that NEC may be familial in origin. ${ }^{90}$ Given this genetic predisposition, finding a genetic marker that is sensitive, specific, and predictive could be a valuable adjunct to early identification and prevention of NEC. ${ }^{91}$ Due to the importance of prematurity, intestinal function, immune defense, inflammatory signaling, and microcirculatory regulation mechanisms, potential variations in many genes could protect or leave a host infant susceptible to NEC. ${ }^{92-103}$ However, NEC is mediated not by genetics alone, but by genetic factors in conjunction with environmental and host-bacteria interactions. Thus, the search for genetic markers appears to have some potential, but sensitivity and specificity for prediction remains elusive largely because of the interaction with non-genetic factors that also play an important role in the pathogenesis of NEC. ${ }^{91}$ Future studies investigating the association of multiple SNPs and NEC may allow for the development of a laboratory genetic test that could predict, when environmental factors are properly assessed, the risk/probability of preterm infants developing NEC and lead to more targeted preventive and therapeutic interventions.

\section{L-ARGININE SUPPLEMENTATION AS PREVENTIVE STRATEGY FOR NEC}

The combination of lost enteral arginine synthetic pathways and high arginine demand in VLBW infants provides the rationale for L-arginine supplementation. ${ }^{104,105}$ However, hypoarginemia in VLBW infants is avoided only when parenteral nutrition arginine content approaches 3 times that of human milk and overall protein intake is high. ${ }^{104,106}$ Moreover, it has been suggested that the intestine only produces arginine if substrate is supplied through enteral nutrition. ${ }^{104,107-109}$ As mentioned elsewhere, data from two small randomized controlled studies ${ }^{110,111}$, pooled in a meta-analysis ${ }^{112}$, suggest that 
enteral L-arginine supplementation reduces the incidence of NEC in preterm infants. One study ${ }^{110}$ administered L-arginine intravenously until enteral feeds reached a predetermined level of the total daily fluid intake, after which point L-arginine was supplemented enterally, while the other focused solely on enteral L-arginine administration. ${ }^{111}$ Large multi-center randomized controlled trials are needed before enteral L-arginine supplementation can become common practice. ${ }^{112}$ Our data from the present thesis suggest that functional genetic variations in the CPS enzyme may alter the effect of L-arginine supplementation in preventing NEC.

\section{ROLE OF OXIDATIVE STRESS ON THE PATHOGENESIS OF NEC}

Oxidative stress refers to a relative surplus of reactive oxygen species (ROS) caused by excessive ROS generation and/or impaired ROS degradation. ${ }^{113}$ Newborns and especially the preterm ones are probably more prone to oxidative stress than later in life. ${ }^{114}$ In 1988 Saugstad coined the term 'oxygen radical disease of neonatology'. ${ }^{115}$ The idea was that oxidative stress may affect different organs, often simultaneously, giving rise to different signs according to the organ most affected. Saugstad included NEC, bronchopulmonary dysplasia (BPD), and retinopathy of prematurity (ROP) in this entity. However, one important consideration is that ROS also have essential functions, for example, within the innate immune response, cellular signaling, control of cellular proliferation and differentiation, oxygen sensing, vascular tone, and angiogenesis. Consequently, interfering with these beneficial roles of ROS in a too broad and unspecific manner can result in pathologies as well, a phenomenon termed as reductive stress. ${ }^{113}$

NADPH oxidases are multicomponent protein complexes containing a catalytic NOX subunit that transfers electrons from NADPH to oxygen, thereby forming ROS. These NOX catalytic subunits represent the only known enzyme family with the sole function to produce ROS. ${ }^{116,117}$ However, the current information on its role in 'oxygen radical disease in neonatology' is scarce. With the increasing understanding on the ubiquitous role of NOX, a large number of allelic variants have been identified for each of the NOX subunits. Some of these variants, particularly those in the p22phox subunit, have been shown to associate with specific functional implications in several disease states. ${ }^{118-}$ 121 Our group is currently investigating several SNPs of NADPH oxidases as potential 
risk factors for NEC as well as other conditions of the prematurity induced by oxidative stress.

\section{UNCOUPLED ENOS IN THE PATHOGENESIS OF NEC}

In situations of reduced availability of the substrate L-arginine or the cofactor $\mathrm{BH}_{4}$, eNOS enzymatic activity becomes uncoupled, resulting in the production of superoxide instead of NO.122 This transformation of eNOS from a protective enzyme to a contributor to oxidative stress has been observed in several animal models of cardiovascular diseases as well as in human patients with endothelial dysfunction. ${ }^{123}$ Similarly, eNOS uncoupling has been suggested to play a role in mesenteric endothelial dysfunction in experimental models ${ }^{124}$ and infants with NEC. ${ }^{125}$ Further understanding of the mechanisms leading to eNOS uncoupling may lead to the design of therapeutic strategies to protect neonatal intestinal vasculature.

\section{NO SYNTHASE-INDEPENDENT PRODUCTION OF NO IN THE NEONATAL INTESTINE}

The L-arginine-NO pathway was considered to be the only source of endogenous NO generation. However, NO has been shown to be generated from the conversion of the nitrate anion from sources in the diet to nitrite through the activity of enteral commensal bacteria. ${ }^{126,127}$ Nitrite is subsequently reduced to form NO through reactions with various nitrite reductase systems, which provide a major source of NO that bypasses the NO-synthase. ${ }^{127}$ This NOS-independent NO production is favored by three conditions unique to the gastrointestinal tract: the selective accumulation of endogenous and dietary-derived nitrogen species, the abundance of commensal bacteria and the extreme redox milieu with gastric acid being an important factor. The effects of $\mathrm{NO}$ and other nitrogen oxides produced by bacteria may include inhibition of pathogen growth, and also effects on the host mucosa including stimulation of mucosal blood flow and mucus generation and modulation of inflammatory responses. ${ }^{127}$

Preterm infants ingest $50 \%$ less nitrate than term infants and plasma nitrite levels were markedly lower in preterm infants than in term infants and adults. ${ }^{128,129}$ Interestingly, It has been suggested that breast milk may exert its protective effects against NEC in part through its previously unrecognized vasodilatory effects by virtue of its rich levels of nitrate. ${ }^{130}$ Nevertheless, plasma nitrite levels of preterm infants before developing 
NEC were similar to healthy preterm controls. ${ }^{129}$ In addition, probiotics may also protect against NEC through NO production. ${ }^{131}$ Interestingly, generation of NO by probiotic bacteria can be counteracted by rapid NO consumption by pathogenic bacterial strains such as E.coli or S. aureus. ${ }^{132,133}$ Gut dysbiosis or disruption of the gut microbiota development, mainly due to organ immaturity, antibiotic use and hospital microbial environment, appears to play a key role in the pathogenesis of NEC. ${ }^{134,135}$ Further studies are warranted in order to clarify the biological role of the bacteria-derived intestinal NO in the prevention and pathogenesis of NEC and how intestinal NO production is affected by gut dysbiosis.

In contrast to the above proposed beneficial effects of the L-arginine-NO and the nitrate-nitrite-NO pathways under physiological conditions, once NEC has reached an acute inflammatory stage, excessive NO production from iNOS contributes to the disease progression. ${ }^{130}$ Consistent with overproduction of NO by iNOS, plasma nitrite and nitrate and urinary nitrite levels in infants developing NEC became significantly elevated, suggesting that urinary nitrite levels could be a useful noninvasive biomarker of NEC. ${ }^{129}$ In addition, the low levels of L-arginine observed in NEC patients might be the consequence of the high utilization of L-arginine by iNOS.

\section{PROBIOTICS, NEC, AND NEONATAL MORBIDITY}

A proposed strategy for the prevention of NEC is the administration of oral probiotics. ${ }^{136,137}$ Probiotic bacteria are live microbial supplements that colonize the gastrointestinal tract and potentially provide benefit to the host. Recent meta-analyses showed that probiotic supplementation reduces the risk of NEC and late onset sepsis (LOS) in preterm infants. ${ }^{136-143}$ In fact, probiotic supplementation is perhaps the best studied yet least used therapy in neonatal medicine. ${ }^{144}$ The issue of whether it is time to change practice and adopt the use of probiotics as a standard of care in preterm infants remains a hot topic for neonatologists. While some advocate a change in practice based on significant reduction in severe NEC, LOS, and all-cause mortality, ${ }^{136,143,144}$ others have raised concerns about the methodology of many of the published trials and the appropriateness of combining them in meta-analyses and advocate for waiting until further data on efficacy and safety in extremely preterm infants are available. ${ }^{145-147}$ In addition, it remains to be determined whether the 
reduction in NEC and/or LOS produced by probiotics translates into a reduction of other complications of the prematurity. Currently, we are conducting a systematic review and meta-analysis in order to evaluate the possible role of probiotic supplementation in reducing the risk of ROP, and BPD in preterm infants. Through meta-regression, we will investigate whether the studies showing a higher rate of protection against NEC and LOS also achieve a higher rate of protection against severe ROP and BPD. 


\section{REFERENCES}

1 Lim, J. C., Golden, J. M. \& Ford, H. R. Pathogenesis of neonatal necrotizing enterocolitis. Pediatr Surg Int 31, 509-518 (2015).

2 Lin, P. W., Nasr, T. R. \& Stoll, B. J. Necrotizing enterocolitis: recent scientific advances in pathophysiology and prevention. Semin Perinato/32, 70-82 (2008).

3 Choi, Y. Y. Necrotizing enterocolitis in newborns: update in pathophysiology and newly emerging therapeutic strategies. Korean J Pediatr 57, 505-513 (2014).

4 Berman, L. \& Moss, R. L. Necrotizing enterocolitis: an update. Seminars in fetal \& neonatal medicine 16, 145-150 (2011).

5 Dominguez, K. M. \& Moss, R. L. Necrotizing enterocolitis. Clinics in perinatology 39, 387-401, (2012).

6 Neu, J. Necrotizing enterocolitis: the mystery goes on. Neonatology 106, 289295 (2014).

7 Gupta, A. \& Paria, A. Etiology and medical management of NEC. Early human development 97, 17-23 (2016).

8 Eaton, S., Rees, C. M. \& Hall, N. J. Current research in necrotizing enterocolitis. Early human development 97, 33-39 (2016).

9 Nankervis, C. A., Giannone, P. J. \& Reber, K. M. The neonatal intestinal vasculature: contributing factors to necrotizing enterocolitis. Semin Perinato/32, 83-91 (2008).

10 Watkins, D. J. \& Besner, G. E. The role of the intestinal microcirculation in necrotizing enterocolitis. Seminars in pediatric surgery 22, 83-87 (2013).

11 Moncada, S. \& Higgs, E. A. The discovery of nitric oxide and its role in vascular biology. British journal of pharmacology 147 Suppl 1, S193-201 (2006).

12 Moncada, S., Palmer, R. M. \& Higgs, E. A. Nitric oxide: physiology, pathophysiology, and pharmacology. Pharmacological reviews 43, 109-142 (1991).

13 Fleming, I. Molecular mechanisms underlying the activation of eNOS. Pflugers Arch (2009).

14 Granger, J. P. Endothelin. American journal of physiology 285, R298-301 (2003). 
15 Reber, K. M., Nankervis, C. A. \& Nowicki, P. T. Newborn intestinal circulation. Physiology and pathophysiology. Clinics in perinatology 29, 23-39 (2002).

16 Nair, J. et al. Fetal and postnatal ovine mesenteric vascular reactivity. Pediatric research 79: 575-582 (2016).

17 Wolfs, T.G. et al. Endotoxin induced chorioamnionitis prevents intestinal development during gestation in fetal sheep. PloS one 4: e5837 (2009).

18 Maltepe, E. \& Saugstad, O. D. Oxygen in health and disease: regulation of oxygen homeostasis-clinical implications. Pediatric research 65, 261-268 (2009).

19 Ross, M. G. Labor and fetal heart rate decelerations: relation to fetal metabolic acidosis. Clinical obstetrics and gynecology 54, 74-82 (2011).

20 Vento, M., Escobar, J., Cernada, M., Escrig, R. \& Aguar, M. The use and misuse of oxygen during the neonatal period. Clinics in perinatology 39, 165-176 (2012).

21 Giussani, D. A. The fetal brain sparing response to hypoxia: physiological mechanisms. The Journal of physiology (2016).

22 Villamor, E., Kessels, C. G., van Suylen, R. J., De Mey, J. G. \& Blanco, C. E. Cardiopulmonary effects of chronic administration of the NO synthase inhibitor L-NAME in the chick embryo. Biology of the neonate 88, 156-163 (2005).

23 Ruijtenbeek, K., Kessels, L. C., De Mey, J. G. \& Blanco, C. E. Chronic moderate hypoxia and protein malnutrition both induce growth retardation, but have distinct effects on arterial endothelium-dependent reactivity in the chicken embryo. Pediatric research 53, 573-579 (2003).

24 Villamor, E. et al. Chronic in ovo hypoxia decreases pulmonary arterial contractile reactivity and induces biventricular cardiac enlargement in the chicken embryo. Am J Physiol Regul Integr Comp Physiol 287, R642-651 (2004).

25 Lindgren, I., Zoer, B., Altimiras, J. \& Villamor, E. Reactivity of chicken chorioallantoic arteries, avian homologue of human fetoplacental arteries. $J$ Physiol Pharmacol 61, 619-628 (2010).

26 Zoer, B. et al. Effects of prenatal hypoxia on pulmonary vascular reactivity in chickens prone to pulmonary hypertension. J Physiol Pharmacol 60, 119-130 (2009). 
27 Agren, P. et al. Developmental changes in endothelium-dependent relaxation of the chicken ductus arteriosus. J Physiol Pharmacol 59, 55-76 (2008).

28 Tibboel, D., van Nie, C. J. \& Molenaar, J. C. The effects of temporary general hypoxia and local ischemia on the development of the intestines: an experimental study. Journal of pediatric surgery 15, 57-62 (1980).

29 Giussani, D. A., Salinas, C. E., Villena, M. \& Blanco, C. E. The role of oxygen in prenatal growth: studies in the chick embryo. The Journal of physiology 585 , 911-917 (2007).

30 Salinas, C. et al. Cardiac and vascular disease prior to hatching in chick embryos incubated at high altitude. Journal of developmental origins of health and disease 1, 60-66 (2010).

31 Salinas, C. et al. Developmental origin of cardiac and vascular disease in chick embryos incubated at high altitude. JDOHaD 1, 60-66 (2010).

32 Salinas, C. E., Blanco, C. E., Villena, M. \& Giussani, D. A. High-altitude hypoxia and echocardiographic indices of pulmonary hypertension in male and female chickens at adulthood. Circulation Journal 78, 1459-1464 (2014).

33 Salinas, C. E., Villena, M., Blanco, C. E. \& Giussani, D. A. Adrenocortical suppression in highland chick embryos is restored during incubation at sea level. High altitude medicine \& biology 12, 79-87 (2011).

34 Moonen, R., Kessels, C., Zimmermann, L. \& Villamor, E. Mesenteric artery reactivity and small intestine morphology in a chicken model of hypoxia-induced fetal growth restriction. Journal of physiology and pharmacology: an official journal of the Polish Physiological Society 63, 601-612 (2012).

35 Lindgren, I., Crossley, D., 2nd, Villamor, E. \& Altimiras, J. Hypotension in the chronically hypoxic chicken embryo is related to the beta-adrenergic response of chorioallantoic and femoral arteries and not to bradycardia. Am J Physiol Regul Integr Comp Physio/ 301, R1161-1168 (2011).

36 Nowicki PT, Dunaway DJ, Nankervis CA, et al. Endothelin-1 in human intestine resected for necrotizing enterocolitis. J Pediatr 146:805-801 (2005).

37 Sbong, S. \& Dzialowski, E. M. Respiratory and cardiovascular responses to acute hypoxia and hyperoxia in internally pipped chicken embryos. Comparative Biochemistry and Physiology Part A: Molecular \& Integrative Physiology 148, 761-768 (2007). 
38 Uni, Z., Tako, E., Gal-Garber, O. \& Sklan, D. Morphological, molecular, and functional changes in the chicken small intestine of the late-term embryo. Poult Sci 82, 1747-1754 (2003).

39 Sabatakou, O., Paraskevakou, E., Tseleni-Balafouta, S., Athanasiadis, A. \& Fasseas, K. The development of the chicken small intestine: a scanning electron microscopy study. J Submicrosc Cytol Pathol 35, 323-330 (2003).

40 Uni, Z., Geyra, A., Ben-Hur, H. \& Sklan, D. Small intestinal development in the young chick: crypt formation and enterocyte proliferation and migration. Br Poult Sci 41, 544-551 (2000).

41 Geyra, A., Uni, Z. \& Sklan, D. Enterocyte dynamics and mucosal development in the posthatch chick. Poult Sci 80, 776-782 (2001).

42 Uni, Z., Smirnov, A. \& Sklan, D. Pre- and posthatch development of goblet cells in the broiler small intestine: effect of delayed access to feed. Poult Sci 82, 320327 (2003).

43 Holmes, K. et al. Localization and control of expression of VEGF-A and the VEGFR-2 receptor in fetal sheep intestines. Pediatric research 63, 143-148 (2008).

44 Neufeld, G., Cohen, T., Gengrinovitch, S. \& Poltorak, Z. Vascular endothelial growth factor (VEGF) and its receptors. The FASEB journal : official publication of the Federation of American Societies for Experimental Biology 13, 9-22 (1999).

45 Molenaar, R. et al. Effect of eggshell temperature and oxygen concentration on survival rate and nutrient utilization in chicken embryos. Poult Sci 89, 2010-2021 (2010).

46 Giussani, D., Spencer, J., Moore, P., Bennet, L. \& Hanson, M. Afferent and efferent components of the cardiovascular reflex responses to acute hypoxia in term fetal sheep. The Journal of physiology 461, 431-449 (1993).

47 Mulder, A., Golde, J., Goor, A. v., Giussani, D. \& Blanco, C. Developmental changes in plasma catecholamine concentrations during normoxia and acute hypoxia in the chick embryo. The Journal of Physiology 527, 593-599 (2000).

48 Mulder, A., Miedema, A., De Mey, J., Giussani, D. A. \& Blanco, C. E. Sympathetic control of the cardiovascular response to acute hypoxemia in the 
chick embryo. American Journal of Physiology-Regulatory, Integrative and Comparative Physiology 282, R1156-R1163 (2002).

49 Mulder, A. L., Miedema, A., De Mey, J. G., Giussani, D. A. \& Blanco, C. E. Sympathetic control of the cardiovascular response to acute hypoxemia in the chick embryo. Am J Physiol Regul Integr Comp Physiol 282, R1156-1163 (2002).

50 Mulder, A. L., van Goor, C. A., Giussani, D. A. \& Blanco, C. E. Alpha-adrenergic contribution to the cardiovascular response to acute hypoxemia in the chick embryo. Am J Physiol Regul Integr Comp Physiol 281, R2004-2010 (2001).

51 Mulder, A. L., Golde, J. M., Goor, A. A., Giussani, D. A. \& Blanco, C. E. Developmental changes in plasma catecholamine concentrations during normoxia and acute hypoxia in the chick embryo. The Journal of physiology 527 Pt 3, 593-599 (2000).

52 Fletcher, A. J., Gardner, D. S., Edwards, C. M. B., Fowden, A. L. \& Giussani, D. A. Development of the ovine fetal cardiovascular defense to hypoxemia towards full term. American Journal of Physiology-Heart and Circulatory Physiology 291, H3023-H3034 (2006).

53 Weir, E., Lopez-Barneo, J., Buckler, K. \& SL, A. Acute oxygen-sensing mechanisms. New England Journal of Medicine. 353, 2042-2055 (2005).

54 Archer, S. \& Michelakis, E. The mechanism(s) of hypoxic pulmonary vasoconstriction: potassium channels, redox $\mathrm{O}(2)$ sensors, and controversies. News Physiol Sci 17, 131-137 (2002).

55 Sylvester, J. T., Shimoda, L. A., Aaronson, P. I. \& Ward, J. P. Hypoxic pulmonary vasoconstriction. Physiol Rev 92, 367-520 (2012).

56 Weir, E. K., Lopez-Barneo, J., Buckler, K. J. \& Archer, S. L. Acute oxygensensing mechanisms. The New England journal of medicine 353, 2042-2055 (2005).

57 Young, C. M., Kingma, S. D. \& Neu, J. Ischemia-reperfusion and neonatal intestinal injury. J Pediatr 158, e25-28 (2011).

58 Shah, V., Lyford, G., Gores, G. \& Farrugia, G. Nitric oxide in gastrointestinal health and disease. Gastroenterology 126:903-913 (2004). 
59 Young, S. L., Evans, K. \& Eu, J. P. Nitric oxide modulates branching morphogenesis in fetal rat lung explants. Am J Physiol Lung Cell Mol Physiol 282, L379-385 (2002).

60 Martinez, A. I., Perez-Arellano, I., Pekkala, S., Barcelona, B. \& Cervera, J. Genetic, structural and biochemical basis of carbamoyl phosphate synthetase 1 deficiency. Molecular genetics and metabolism 101, 311-323 (2010).

61 Ali, E. Z. et al. Carbamoylphosphate synthetase 1 (CPS1) deficiency: clinical, biochemical, and molecular characterization in Malaysian patients. European journal of pediatrics 175, 339-346 (2016).

62 Summar, M. L. et al. Environmentally determined genetic expression: clinical correlates with molecular variants of carbamyl phosphate synthetase I. Molecular genetics and metabolism 81 Suppl 1, S12-19 (2004).

63 Summar, M. L. et al. Characterization of genomic structure and polymorphisms in the human carbamyl phosphate synthetase I gene. Gene 311, 51-57 (2003).

64 Summar, M. L. et al. Relationship between carbamoyl-phosphate synthetase genotype and systemic vascular function. Hypertension 43, 186-191 (2004).

65 Pearson, D. L. et al. Neonatal pulmonary hypertension--urea-cycle intermediates, nitric oxide production, and carbamoyl-phosphate synthetase function. The New England journal of medicine 344, 1832-1838 (2001).

66 Kallianpur, A. R. et al. The hemochromatosis C282Y allele: a risk factor for hepatic veno-occlusive disease after hematopoietic stem cell transplantation. Bone marrow transplantation 35, 1155-1164 (2005).

67 Canter, J. A. et al. Genetic variation in the mitochondrial enzyme carbamylphosphate synthetase I predisposes children to increased pulmonary artery pressure following surgical repair of congenital heart defects: a validated genetic association study. Mitochondrion 7, 204-210 (2007).

68 Wu, G., Jaeger, L. A., Bazer, F. W. \& Rhoads, J. M. Arginine deficiency in preterm infants: biochemical mechanisms and nutritional implications. The Journal of nutritional biochemistry 15, 442-451 (2004).

69 Wu, S. F., Caplan, M. \& Lin, H. C. Necrotizing enterocolitis: old problem with new hope. Pediatr Neonatol 53, 158-163 (2012).

70 Amin, H. J. et al. Arginine supplementation prevents necrotizing enterocolitis in the premature infant. J Pediatr 140, 425-431 (2002). 
71 Becker, R. M. et al. Reduced serum amino acid concentrations in infants with necrotizing enterocolitis. J Pediatr 137, 785-793 (2000).

72 Celik, I. H., Demirel, G., Canpolat, F. E. \& Dilmen, U. Reduced plasma citrulline levels in low birth weight infants with necrotizing enterocolitis. Journal of clinical laboratory analysis 27, 328-332 (2013).

73 Richir, M. C. et al. Low plasma concentrations of arginine and asymmetric dimethylarginine in premature infants with necrotizing enterocolitis. Br J Nutr $\mathbf{9 7}$, 906-911 (2007).

74 Zamora, S. A. et al. Plasma L-arginine concentrations in premature infants with necrotizing enterocolitis. J Pediatr 131, 226-232 (1997).

75 Polycarpou, E. et al. Enteral L-arginine supplementation for prevention of necrotizing enterocolitis in very low birth weight neonates: a double-blind randomized pilot study of efficacy and safety. JPEN J Parenter Enteral Nutr 37, 617-622 (2013).

76 Mitchell, K. et al. Arginine supplementation in prevention of necrotizing enterocolitis in the premature infant: an updated systematic review. BMC Pediatr 14, 226 (2014).

77 Sullivan, J. C. \& Pollock, J. S. Coupled and uncoupled NOS: separate but equal? Uncoupled NOS in endothelial cells is a critical pathway for intracellular signaling. Circulation research 98, 717-719 (2006).

78 Premkumar, M. H. et al. Argininosuccinate lyase in enterocytes protects from development of necrotizing enterocolitis. Am J Physiol Gastrointest Liver Physiol 307, G347-354 (2014).

79 Kohler, E. S. et al. The human neonatal small intestine has the potential for arginine synthesis; developmental changes in the expression of argininesynthesizing and -catabolizing enzymes. BMC Dev Biol 8, 107 (2008).

80 Nathanielsz, P. W. Animal models that elucidate basic principles of the developmental origins of adult diseases. Ilar Journal 47, 73-82 (2006).

81 Sartori, C. et al. Augmented vasoreactivity in adult life associated with perinatal vascular insult. Lancet 353, 2205-2207 (1999).

82 Zoer, B. et al. Effects of prenatal hypoxia on pulmonary vascular reactivity in chickens prone to pulmonary hypertension. J Physiol Pharmacol 60, 119-130 (2009). 
83 Vento, M., Escobar, J., Cernada, M., Escrig, R. \& Aguar, M. The use and misuse of oxygen during the neonatal period. Clinics in perinatology 39, 165-176 (2012).

84 Escobar, J. et al. Prolonging in utero-like oxygenation after birth diminishes oxidative stress in the lung and brain of mice pups. Redox biology 1, 297-303 (2013).

85 Saugstad, O. D. Optimal oxygenation at birth and in the neonatal period. Neonatology 91, 319-322 (2007).

86 Maltepe, E. \& Saugstad, O. D. Oxygen in health and disease: regulation of oxygen homeostasis-clinical implications. Pediatric research 65, 261-268 (2009).

$87 \mathrm{Li}$, C. \& Jackson, R. M. Reactive species mechanisms of cellular hypoxiareoxygenation injury. American Journal of Physiology-Cell Physiology 282, C227-C241 (2002).

$88 \mathrm{Ng}, \mathrm{C}$. D., Deshpande, S. S., Irani, K. \& Alevriadou, B. R. Adhesion of flowing monocytes to hypoxia-reoxygenation-exposed endothelial cells: role of Rac1, ROS, and VCAM-1. American Journal of Physiology-Cell Physiology 283, C93C102 (2002).

89 Mohazzab-H, K. M., Kaminski, P. M., Fayngersh, R. P. \& Wolin, M. S. Oxygenelicited responses in calf coronary arteries: role of $\mathrm{H} 2 \mathrm{O} 2$ production via NADHderived superoxide. American Journal of Physiology-Heart and Circulatory Physiology 270, H1044-H1053 (1996).

90 Bhandari, V. et al. Familial and genetic susceptibility to major neonatal morbidities in preterm twins. Pediatrics 117, 1901-1906 (2006).

91 Young, C., Sharma, R., Handfield, M., Mai, V. \& Neu, J. Biomarkers for infants at risk for necrotizing enterocolitis: clues to prevention? Pediatr Res 65, 91R97R (2009).

92 Lin, P. W., Nasr, T. R. \& Stoll, B. J. Necrotizing enterocolitis: recent scientific advances in pathophysiology and prevention. Semin Perinato/32, 70-82 (2008).

$93 \mathrm{Ng}, \mathrm{P}$. C. Biomarkers of necrotising enterocolitis. Seminars in fetal \& neonatal medicine 19, 33-38, (2014).

94 Sampath, V. et al. SIGIRR Genetic Variants in Premature Infants With Necrotizing Enterocolitis. Pediatrics 135, e1530-1534 (2015). 
95 Zhou, W. et al. Association of neonatal necrotizing enterocolitis with myeloid differentiation-2 and GM2 activator protein genetic polymorphisms. Mol Med Rep 12, 974-980 (2015).

96 Heninger, E. et al. Genetic variants of the interleukin-18 promoter region (-607) influence the course of necrotising enterocolitis in very low birth weight neonates. European journal of pediatrics 161, 410-411 (2002).

97 Bokodi, G., Derzbach, L., Banyasz, I., Tulassay, T. \& Vasarhelyi, B. Association of interferon gamma $\mathrm{T}+874 \mathrm{~A}$ and interleukin 12 p40 promoter CTCTAA/GC polymorphism with the need for respiratory support and perinatal complications in low birthweight neonates. Archives of disease in childhood. Fetal and neonatal edition 92, F25-29 (2007).

98 Prencipe, G. et al. Association between mannose-binding lectin gene polymorphisms and necrotizing enterocolitis in preterm infants. $J$ Pediatr Gastroenterol Nutr 55, 160-165 (2012).

99 Sampath, V. et al. The NFKB1 (g.-24519delATTG) variant is associated with necrotizing enterocolitis (NEC) in premature infants. J Surg Res 169, e51-57 (2011).

100 Treszl, A. et al. Lower prevalence of IL-4 receptor alpha-chain gene G variant in very-low-birth-weight infants with necrotizing enterocolitis. Journal of pediatric surgery 38, 1374-1378 (2003).

101 Henderson, G. et al. Cytokine gene polymorphisms in preterm infants with necrotising enterocolitis: genetic association study. Archives of disease in childhood. Fetal and neonatal edition 94, F124-128 (2009).

102 Franklin, A. L. et al. Are Immune Modulating Single Nucleotide Polymorphisms Associated with Necrotizing Enterocolitis? Sci Rep 5, 18369 (2015).

103 Bányász, I. et al. Genetic polymorphisms for vascular endothelial growth factor in perinatal complications. European cytokine network 17, 266-270 (2006).

104 Morgan, C. \& Burgess, L. High protein intake does not prevent low plasma levels of conditionally essential amino acids in very preterm infants receiving parenteral nutrition. Journal of Parenteral and Enteral Nutrition, 0148607115594009 (2015). 
105 Wu, G., Jaeger, L. A., Bazer, F. W. \& Rhoads, J. M. Arginine deficiency in preterm infants: biochemical mechanisms and nutritional implications. The Journal of nutritional biochemistry 15, 442-451 (2004).

106 Burgess, L., Morgan, C., Mayes, K. \& Tan, M. Plasma arginine levels and blood glucose control in very preterm infants receiving 2 different parenteral nutrition regimens. Journal of Parenteral and Enteral Nutrition 38, 243-253 (2014).

107 Kohler, E. S. et al. The human neonatal small intestine has the potential for arginine synthesis; developmental changes in the expression of argininesynthesizing and -catabolizing enzymes. BMC Dev Biol 8, 107 (2008).

108 Wu, G., Jaeger, L. A., Bazer, F. W. \& Rhoads, J. M. Arginine deficiency in preterm infants: biochemical mechanisms and nutritional implications. The Journal of nutritional biochemistry 15, 442-451 (2004).

109 Tomlinson, C., Rafii, M., Sgro, M., Ball, R. O. \& Pencharz, P. Arginine is synthesized from proline, not glutamate, in enterally fed human preterm neonates. Pediatric research 69, 46-50 (2011).

110 Amin, H. J. et al. Arginine supplementation prevents necrotizing enterocolitis in the premature infant. J Pediatr 140, 425-431 (2002).

111 Polycarpou, E. et al. Enteral L-Arginine Supplementation for Prevention of Necrotizing Enterocolitis in Very Low Birth Weight Neonates A Double-Blind Randomized Pilot Study of Efficacy and Safety. Journal of Parenteral and Enteral Nutrition, 0148607112471561 (2013).

112 Mitchell, K. et al. Arginine supplementation in prevention of necrotizing enterocolitis in the premature infant: an updated systematic review. BMC pediatrics 14, 1 (2014).

113 Radermacher, K. A. et al. Neuroprotection After Stroke by Targeting NOX4 As a Source of Oxidative Stress. Antioxidants \& redox signaling, 18:1418-1427 (2013).

114 Saugstad, O. D. Oxidative stress in the newborn--a 30-year perspective. Biology of the neonate 88, 228-236 (2005).

115 Saugstad, O. D. Oxygen radical disease in neonatology. Seminars in Neonatology 3, 229-238 (1988). 
116 Kleikers, P. W. et al. NADPH oxidases as a source of oxidative stress and molecular target in ischemia/reperfusion injury. J Mol Med (Berl) 90:1391-1406 (2012).

117 Altenhofer, S. et al. The NOX toolbox: validating the role of NADPH oxidases in physiology and disease. Cellular and molecular life sciences : CMLS 69, 23272343 (2012).

118 Mehranpour, P. et al. The C242T CYBA polymorphism as a major determinant of NADPH oxidase activity in patients with cardiovascular disease. Cardiovascular \& hematological agents in medicinal chemistry 7, 251-259 (2009).

119 Gozal, D. et al. Cognitive function in prepubertal children with obstructive sleep apnea: a modifying role for NADPH oxidase p22 subunit gene polymorphisms? Antioxidants \& redox signaling 16, 171-177 (2012).

120 Brandes, R. P., Weissmann, N. \& Schroder, K. NADPH oxidases in cardiovascular disease. Free Radic Biol Med 49, 687-706 (2010).

121 Cave, A. C. et al. NADPH oxidases in cardiovascular health and disease. Antioxidants \& redox signaling 8, 691-728 (2006).

122 Sullivan, J. C. \& Pollock, J. S. Coupled and uncoupled NOS: separate but equal? Uncoupled NOS in endothelial cells is a critical pathway for intracellular signaling. Circulation research 98, 717-719 (2006).

123 Förstermann, U. \& Münzel, T. Endothelial nitric oxide synthase in vascular disease from marvel to menace. Circulation 113, 1708-1714 (2006).

124 Whitehouse, J. S. et al. Mesenteric nitric oxide and superoxide production in experimental necrotizing enterocolitis. Journal of Surgical Research 161, 1-8 (2010).

125 Nowicki, P. T. et al. Endothelial nitric oxide synthase in human intestine resected for necrotizing enterocolitis. The Journal of pediatrics 150, 40-45 (2007).

126 Sobko, T. et al. Gastrointestinal bacteria generate nitric oxide from nitrate and nitrite. Nitric Oxide 13, 272-278 (2005).

127 Lundberg, J. O. \& Weitzberg, E. Biology of nitrogen oxides in the gastrointestinal tract. Gut, 62:616-629 (2013). 
128 Jones, J. A., Hopper, A. O., Power, G. G. \& Blood, A. B. Dietary intake and bioactivation of nitrite and nitrate in newborn infants. Pediatric research $77,173-$ 181 (2014).

129 Pun, P. et al. Changes in plasma and urinary nitrite after birth in premature infants at risk for necrotizing enterocolitis. Pediatric research 79, 432-437 (2015).

130 Yazji, I. et al. Endothelial TLR4 activation impairs intestinal microcirculatory perfusion in necrotizing enterocolitis via eNOS-NO-nitrite signaling. Proceedings of the National Academy of Sciences 110, 9451-9456 (2013).

131 Yarullina, D. R. et al. Role of Nitric Oxide Produced by Lactobacilli in Relaxation of Intestinal Smooth Muscles. Bull Exp Biol Med 160, 343-346 (2016).

132 Sobko, T. et al. Generation of NO by probiotic bacteria in the gastrointestinal tract. Free Radic Biol Med 41, 985-991 (2006).

133 Sobko, T. Influence of the microflora on gastrointestinal nitric oxide generation: Studies in newborn infants and germ-free animals. (Institutionen för fysiologi och farmakologi/Department of Physiology and Pharmacology, 2006).

134 Warner, B. B. \& Tarr, P. I. Necrotizing enterocolitis and preterm infant gut bacteria. Semin Fetal Neonatal Med, doi:10.1016/j.siny.2016.06.001 (2016).

135 Torrazza, R. M. \& Neu, J. The altered gut microbiome and necrotizing enterocolitis. Clin Perinatol 40, 93-108 (2013).

136 AlFaleh, K. \& Anabrees, J. Probiotics for prevention of necrotizing enterocolitis in preterm infants. Cochrane Database Syst Rev 4, CD005496 (2014).

$137 \mathrm{Neu}, \mathrm{J}$. Probiotics and necrotizing enterocolitis. Clinics in perinatology 41, 967978 (2014).

138 Lau, C. S. \& Chamberlain, R. S. Probiotic administration can prevent necrotizing enterocolitis in preterm infants: A meta-analysis. J Pediatr Surg 50, 1405-1412 (2015).

139 Wang, Q., Dong, J. \& Zhu, Y. Probiotic supplement reduces risk of necrotizing enterocolitis and mortality in preterm very low-birth-weight infants: an updated meta-analysis of 20 randomized, controlled trials. J Pediatr Surg 47, 241-248 (2012).

140 Aceti, A. et al. Probiotics for prevention of necrotizing enterocolitis in preterm infants: systematic review and meta-analysis. Ital J Pediatr 41, 89 (2015). 
141 Olsen, R., Greisen, G., Schroder, M. \& Brok, J. Prophylactic Probiotics for Preterm Infants: A Systematic Review and Meta-Analysis of Observational Studies. Neonatology 109, 105-112 (2016).

142 Zhang, G. Q., Hu, H. J., Liu, C. Y., Shakya, S. \& Li, Z. Y. Probiotics for Preventing Late-Onset Sepsis in Preterm Neonates: A PRISMA-Compliant Systematic Review and Meta-Analysis of Randomized Controlled Trials. Medicine (Baltimore) 95, e2581 (2016).

143 Rao, S. C., Athalye-Jape, G. K., Deshpande, G. C., Simmer, K. N. \& Patole, S. K. Probiotic Supplementation and Late-Onset Sepsis in Preterm Infants: A Meta-analysis. Pediatrics 137, 1-16 (2016).

144 Janvier, A., Malo, J. \& Barrington, K. J. Cohort study of probiotics in a North American neonatal intensive care unit. The Journal of pediatrics 164, 980-985 (2014).

145 Neu, J. Routine probiotics for premature infants: let's be careful! The Journal of pediatrics 158, 672 (2011).

146 Millar, M., Wilks, M., Fleming, P. \& Costeloe, K. Should the use of probiotics in the preterm be routine? Archives of Disease in Childhood-Fetal and Neonatal Edition 97, F70-F74 (2012).

147 Mihatsch, W. A. et al. Critical systematic review of the level of evidence for routine use of probiotics for reduction of mortality and prevention of necrotizing enterocolitis and sepsis in preterm infants. Clin Nutr 31, 6-15 (2012). 
Chapter 9 NEDERLANDSE SAMENVATTING

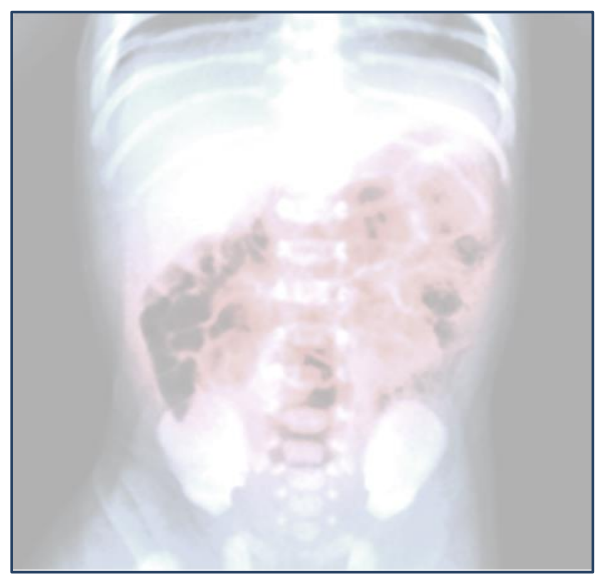


Necrotiserende enterocolitis (NEC) is een ernstige darmontsteking waarbij delen van de darm kunnen afsterven (necrose). Zowel de dunne darm als de dikke darm kunnen aangedaan zijn. Soms is een klein stukje darm aangedaan, maar het kan ook een veel groter deel betreffen.

NEC is één van de meest ernstige en ook levensbedreigende, acute aandoeningen die we zien op de Neonatale Intensive Care Unit (NICU) en komt vooral voor bij te vroeg geboren kinderen (prematuren) en pasgeborenen met een heel laag geboortegewicht. Hoe korter de zwangerschapsduur en hoe lager het geboortegewicht, hoe groter het risico op NEC. Ongeveer zeven procent van de kinderen die geboren is bij een zwangerschap van minder dan 32 weken krijgt NEC. De sterftekans bij kinderen die minder wegen dan 1500 gram is $5-30 \%$ en de kans op overlijden neemt toe naarmate het geboortegewicht lager is. Vaak overlijden de kinderen met ernstige NEC al binnen 24 uur. NEC is dus een ziektebeeld met een hoge sterfte op de korte termijn, maar ook ernstige gevolgen op de lange termijn.

De oorzaak van NEC is niet geheel bekend, maar prematuriteit, groeivertraging tijdens de zwangerschap, voeding en bacteriën spelen een rol. Daarnaast is onderontwikkelde regulatie van de bloedtoevoer naar de darmen een van de veronderstelde mechanismen. Echter, de kennis van de ontwikkeling van de regulatie van bloedtoevoer naar de darmen is nog beperkt.

In dit proefschrift onderzochten we de rol van de regulatie van de bloedtoevoer naar de darmen en we benaderden dit probleem vanuit 2 perspectieven. In het eerste deel onderzochten we de ontwikkeling van darmbloedvatreactiviteit tijdens een normale zwangerschap en tijdens een zwangerschap waarbij zuurstoftekort (hypoxie) optreedt. In het tweede deel onderzochten we de rol van L-arginine, een aminozuur dat de bron is voor de productie van stikstofmonoxide (NO), bij de ontwikkeling van NEC. 


\section{DEEL 1: DE ONTWIKKELING VAN DARMBLOEDVATREACTIVITEIT TIJDENS EEN NORMALE ZWANGERSCHAP EN TIJDENS EEN ZWANGERSCHAP WAARBIJ ZUURSTOFTEKORT (HYPOXIE) OPTREEDT.}

In dit deel hebben we de kip als model genomen voor de ontwikkelingsbiologie van de darmbloedvaten. Kippen zijn warmbloedige gewervelde dieren, die biologisch gezien veel overeenkomsten vertonen met zoogdieren. De kip is een ideaal model voor het onderzoeken van de eigenschappen van darmbloedvaten en andere bloedvaten. Bovendien is het relatief eenvoudig om de omstandigheden waarin het kippenembryo zich ontwikkelt aan te passen, omdat deze ontwikkeling plaatsvindt buiten het moederdier. Zo is het bijvoorbeeld mogelijk om situaties met verminderde aanvoer van zuurstof of voedingsstoffen na te bootsen, of de invloed van bijvoorbeeld medicatie op de embryogenese te bestuderen.

De bloedvatreactiviteit bij zoogdieren (foetus en pasgeborene) wordt geregeld door een balans tussen een bloedvatverwijder (NO) en een bloedvatvernauwer (ET-1). Een disbalans tussen NO en ET-1 zou de onrijpe darm bij prematuren mogelijk voorbestemmen om NEC te ontwikkelen. De balans zorgt na de geboorte bij zoogdieren voor bloedvatverwijding, om zo een toegenomen bloedvoorziening naar de darmen te hebben. Dit is belangrijk voor de werking van de darmen na de geboorte.

In hoofdstuk 2 worden de resultaten beschreven van de normale ontwikkeling van darmbloedvatreactiviteit gedurende de foetale ontwikkeling in het ei ("zwangerschap") en na het uitkomen van het ei ("geboorte"). We vonden dat, net zoals bij zoogdieren, de bloedvatverwijdende mechanismen zich eerder ontwikkelen dan de bloedvatvernauwende mechanismen van darmbloedvaten. Daarnaast is er bij kippen laat in de "zwangerschap" de meeste disbalans tussen bloedvatverwijdende mediatoren en bloedvatvernauwende mediatoren. Dit in het voordeel van de laatst genoemden. Na de "geboorte", als er voeding wordt gegeven, verschuift deze balans in het voordeel van bloedvatverwijding. Dit is nodig om na de geboorte voedingsstoffen op te kunnen nemen via de darmen. Bij kippen zeer vroeg in de "zwangerschap" is er bij de darmbloedvaten met name een situatie van bloedvatverwijding. 
In hoofdstuk 3 beschrijven we hoe chronisch zuurstoftekort (hypoxie) gedurende incubatie van de eieren de darmbloedvatreactiviteit en darmwandontwikkeling van kippenembryo's en pas uitgekomen kuikens beïnvloedt. Chronische hypoxie veroorzaakte een overgevoeligheid van de darmbloedvaten aan ET-1, zelfs in de eerste levensuren na "de geboorte". Dit veroorzaakte een bloedvatvernauwing gedurende de eerste levensuren van het kuiken. Dit is mogelijk ongunstig in het licht van voeding van pasgeborenen. Op de darmwandontwikkeling had chronische hypoxie enkel subtiele effecten die verdwenen waren bij 1 dag oude kuikens.

Zowel bij zoogdier foetussen als kippenembryo's zorgt acuut zuurstoftekort voor een herverdeling van de bloedvoorziening naar vitale organen zoals hart, hersenen en bijnieren. Hierdoor gaat minder bloed naar de niet-vitale organen zoals o.a. darmen door bloedvatvernauwing van deze bloedvaten. In hoofdstuk 4 tonen we aan dat bloedvatvernauwing bij zuurstoftekort een intrinsieke eigenschap is van de darmbloedvaten bij de kip laat in de "zwangerschap" en in de periode rondom de "geboorte". Na de "geboorte" is deze eigenschap aanwezig gedurende de eerste dagen. Vroeg in de "zwangerschap" is dit niet aanwezig bij darmbloedvaten van de kip.

\section{DEEL II: DE ROL VAN L-ARGININE EN STIKSTOFMONOXIDE (NO), BIJ DE ONTWIKKELING VAN NEC}

$\mathrm{NO}$ is belangrijk voor vele processen in de darm. De bron voor de productie van NO is het aminozuur L-arginine. L-arginine is een van de producten die gemaakt worden in de ureumcyclus. Carbamoyl phosphate synthetase 1 (CPS1) is het allesbepalende eerste enzym in de ureumcyclus. Bepaalde genetische variaties in dit enzym worden geassocieerd met verminderde NO productie.

In hoofdstuk 5 beschrijven we een genetische variatie in het CPS1 gen (p.Thr1406Asn polymorfisme) die geassocieerd lijkt te zijn met het risico op het ontwikkelen van NEC in een kleine groep prematuren. 
Echter in de wetenschap is dit niet voldoende om aan te tonen dat er echt een associatie aanwezig is. Daarvoor is onderzoek nodig bij een grote groep prematuren.

In hoofdstuk 6 beschrijven we de resultaten van een grote internationale studie naar de mogelijke associatie tussen het p.Thr1406Asn polymorfisme en het optreden van NEC. Deze studie betrof 477 prematuren uit 4 NICU's (Las Palmas de Gran Canaria, Spanje; Mantova, Italië; Milaan, Italië en Maastricht, Nederland). In deze studie vinden we opnieuw bewijs dat er een associatie is tussen p.Thr1406Asn polymorfisme en het risico om NEC te ontwikkelen bij deze groep prematuren.

In een studie in 2001 werd reeds een relatie gevonden tussen het p.Thr1406Asn polymorfisme in het CPS1 gen en L-arginine bloedspiegels bij voldragen pasgeborenen. In hoofdstuk 7 kijken we of bij prematuren deze relatie ook aan te tonen is. In onze studie bij prematuren vonden we echter geen relatie tussen het p.Thr1406Asn polymorfisme en L-arginine bloedspiegels vlak na de geboorte. Mogelijk dat dit wel het geval is later in het leven. 
Chapter 10

VALORISATION ADDENDUM

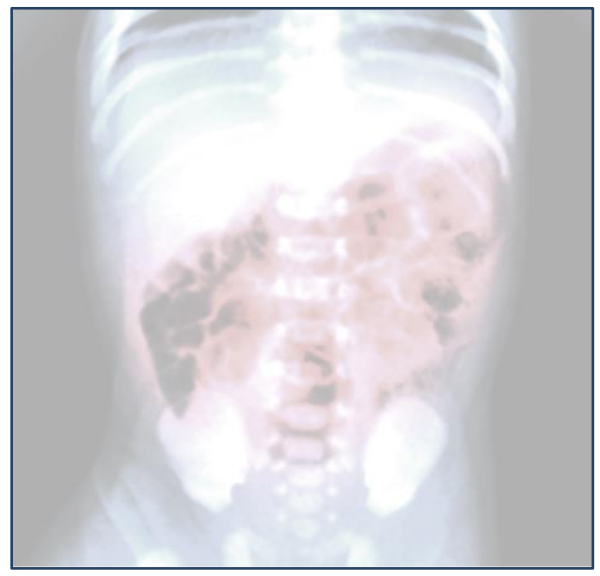




\section{RELEVANCE}

Necrotizing enterocolitis (NEC) is an inflammatory intestinal disorder primarily seen in preterm infants, characterized by variable damage to the intestinal tract, ranging from mucosal injury to full-thickness necrosis and perforation. ${ }^{1-5} \mathrm{NEC}$ remains a leading cause of morbidity and mortality in neonatal intensive care units. The overall incidence of NEC is about 1 in 1000 live births, but occurs in up to $7-11 \%$ of very low birth weight (VLBW, birth weight $<1500 \mathrm{~g}$ ) infants. Mortality is $15-30 \%$ and is higher with lower birth weight and earlier gestational age.

The clinical picture of NEC includes distended abdomen, peri-umbilical erythema, bloody stools, feeding intolerance and overall instability of the infant. The modified Bell's staging criteria ${ }^{6,7}$ are used to stratify NEC in 3 different stages of increasing severity. This staging system uses systemic signs, findings in the abdominal examination, and radiographic clues. In NEC Bell's stage I there are non-specific symptoms as in feed intolerance and non-gut related sepsis. However, these infants can rapidly progress to stage III. Bell's stage II and stage III indicate definite and advanced stages of the disease respectively. Intestinal perforation is defined as stage IIIB. 8

Early detection and initiation of treatment in NEC are key factors in its course and prognosis. Basic principles of medical management of NEC include bowel rest, antibiotics, routine gastric decompression and supportive care. ${ }^{8}$ Pneumoperitoneum due to intestinal perforation is an absolute indication for surgery. However, there are also some relative indication for surgery in NEC. ${ }^{9}$

Survivors may be left with significant sequelae, which include gastrointestinal complications such as a short gut syndrome, but also stoma related morbidity, strictures, and central venous catheter related problems (for example sepsis). ${ }^{5,9}$

NEC is a disease associated with a prolongation of the NICU stay. Both medical and surgical NEC markedly increase the costs of hospitalization. ${ }^{5,10}$ It is estimated that the average charge in the United States associated with a case of surgical NEC is 
$\$ 400.000$ to $\$ 500.000$. One prospective study, which considered hospital cost, found that the costs of surgical NEC were between $\$ 300000$ and $\$ 660000 .{ }^{11}$

The long-term outcome after hospital discharge was evaluated in several studies. NEC is associated with significantly worse neurodevelopmental outcome (both motor and cognitive outcome) early in live ${ }^{12}$ and at school age than prematurity alone. ${ }^{13-15}$ Presence of advanced NEC and need for surgery increase the risk of neurological impairment. ${ }^{14}$ This is not only at early school age, but also educational outcome at 11 years is worse. ${ }^{16}$ Also NEC survivors demonstrated significant long-term consequences of gut function (presence of stoma, admission for bowel problems and continuing medical care for gut-related problems). ${ }^{15}$

Obviously preventive strategies that reduce the prevalence of NEC during the NICU hospitalization are both a clinical and an economic priority for society. This can only be achieved with a more profound understanding of the pathophysiological mechanisms leading to NEC. One of the suspected ethiopathogenic mechanisms is an immature mesenteric circulatory regulation. Understanding of the mechanisms that regulate the mesenteric artery (MA) vascular tone, could have clinical implications and lead to better management options and better neonatal outcome.

Significant progress in our understanding of the cardiovascular physiology and pathophysiology has been achieved with the use of animal models. However, mammalian models are complex because the fetal/placental circulation has to be exposed to intervention only through complex surgery and experimental manipulations affect both the mother and the fetus. Therefore, there is a need for additional models, addressing these limitations. ${ }^{17}$ The chicken (Gallus gallus) embryo represent an excellent model for investigating developmental physiology of the cardiovascular system. ${ }^{18}$ Chicken embryos have a mammalian-like circulation, with an extraembryonic circuit involved in the gas exchange (the chorioallantois), analogous to the placenta. In contrast to mammals, which depend on a continuous transference of nutrients from the maternal circulation to the developing fetus, all the nutrients required for the formation and growth of the chicken embryo are pre-packaged in the egg at the time of laying. ${ }^{19}$ As the chicken embryo develops outside the mother, the number of experimental animals is divided by two, and the effects of external stresses on 
cardiovascular development can be studied without interferences of maternal hormonal, metabolic, or hemodynamic alterations.

\section{INNOVATION}

With this present thesis, we focused on role of the vascular system in the pathogenesis of NEC and approached the problem from two perspectives. In the first part, we investigated the developmental changes in mesenteric arterial reactivity. In the second part, we analyzed the role of L-arginine, the precursor for the synthesis of $\mathrm{NO}$, on the pathogenesis of NEC.

Only a limited number of observations have been made on the development of fetal intestinal circulation in mammals. In the chicken embryo only relative immature vessels were investigated without analyzing further maturation. Our publication described the maturational differences in the reactivity of MAs isolated from chicken embryos at several stages and hatchlings. To this knowledge we added the evidence that chronic moderate hypoxia during incubation results in subtle but significant alterations in chicken MA reactivity, small intestine morphology and VEGF expression. To the best of our knowledge, no study has investigated the response to hypoxia in isolated fetal MAs and even the data in neonatal MAs are scarce. We are the first showing that the local response to hypoxia was absent in the less mature MA, indirectly support the hypothesis that the immature intestine is less susceptible to direct hypoxic damage. In fact, the publication of our findings has already attracted the attention of other investigators in the field of MA developmental biology. ${ }^{20,21}$

The use of animal subjects is fundamental to the advancement of biomedical research and practice, at least until viable replacements are found. Equally important are the ethical guidelines and welfare protocols that shape the conduct of such research. Animal welfare guidelines and legislation still emphasises Russell and Burch's 'three R's': reduction (of the number of animals used), refinement (of testing procedures to minimise suffering), and replacement (of animal models with alternatives). ${ }^{22}$ 
L-arginine is the substrate for NO production and several studies demonstrated that plasma arginine concentrations are decreased in infants with NEC. Nevertheless, the mechanisms explaining this relative deficiency of L-arginine in infants with NEC are far from being understood. Several studies demonstrated the association of the CPS1 p.Thr1406Asn genotype with clinical situations where endogenous NO production is critically important. But the role of this polymorphism in NEC was not investigated until our retrospective case-control study and recent multicenter, prospective cohort study. We provided evidence that a functional variant of the CPS1 gene may contribute to NEC susceptibility. Even in the recent literature about NEC, our retrospective casecontrol study from several years ago is cited. Last but not least, our data did not confirm the hypothesis that in a population of preterm infants, in whom the urea cycle is not fully developed, genetically determined variations in CPS1 function would induce further changes in L-arginine levels. We did not find differences in urea cycle intermediates (i.e. citrulline and arginine) between the different CPS1 genotypes.

In conclusion, this thesis adds understanding of the basic mechanisms of normal and altered functional and structural development of the mesenteric vessels to the current knowledge about MA vasoreactivity. This, as well as inter-species differences in mesenteric circulation, may provide insights into human intestinal disease.

Also this thesis provides further evidence that a functional variant of the CPS1 gene may contribute to NEC susceptibility. This brings us one step closer to developing a laboratory genetic test that could predict, when environmental factors are properly assessed, the risk/probability of preterm infants developing NEC and leads to more targeted therapies and also preventive strategies. This is important to reach the ultimate goal reducing the prevalence of NEC during the NICU hospitalization. 


\section{REFERENCES}

1 Berman, L. \& Moss, R. L. Necrotizing enterocolitis: an update. Seminars in fetal \& neonatal medicine 16, 145-150 (2011).

2 Choi, Y. Y. Necrotizing enterocolitis in newborns: update in pathophysiology and newly emerging therapeutic strategies. Korean J Pediatr 57, 505-513 (2014).

3 Dominguez, K. M. \& Moss, R. L. Necrotizing enterocolitis. Clinics in perinatology 39, 387-401 (2012).

$4 \quad$ Lim, J. C., Golden, J. M. \& Ford, H. R. Pathogenesis of neonatal necrotizing enterocolitis. Pediatr Surg Int 31, 509-518 (2015).

5 Neu, J. Necrotizing enterocolitis: the mystery goes on. Neonatology 106, 289295 (2014).

6 Walsh, M. C. \& Kliegman, R. M. Necrotizing enterocolitis: treatment based on staging criteria. Pediatric clinics of North America 33, 179-201 (1986).

7 Lee, J. S. \& Polin, R. A. Treatment and prevention of necrotizing enterocolitis. Seminars in neonatology : SN 8, 449-459 (2003).

8 Gupta, A. \& Paria, A. Etiology and medical management of NEC. Early human development 97, 17-23 (2016).

9 Thakkar, H. S. \& Lakhoo, K. The surgical management of necrotising enterocolitis (NEC). Early human development 97, 25-28 (2016).

10 Johnson, T. J., Patel, A. L., Jegier, B. J., Engstrom, J. L. \& Meier, P. P. Cost of morbidities in very low birth weight infants. $J$ Pediatr 162, 243-249 e241 (2013).

11 Stey, A. et al. Outcomes and costs of surgical treatments of necrotizing enterocolitis. Pediatrics 135, e1190-1197 (2015).

12 So, S. et al. Early neurodevelopmental outcomes of infants with intestinal failure. Early human development 101, 11-16 (2016).

13 Roze, E. et al. Functional impairments at school age of children with necrotizing enterocolitis or spontaneous intestinal perforation. Pediatric research 70, 619-625 (2011).

14 Rees, C. M., Pierro, A. \& Eaton, S. Neurodevelopmental outcomes of neonates with medically and surgically treated necrotizing enterocolitis. 
Archives of disease in childhood. Fetal and neonatal edition 92, F193-198 (2007).

15 Pike, K. et al. Outcomes at 7 years for babies who developed neonatal necrotising enterocolitis: the ORACLE Children Study. Archives of disease in childhood. Fetal and neonatal edition 97, F318-322 (2012).

16 Johnson, S., Wolke, D., Hennessy, E. \& Marlow, N. Educational outcomes in extremely preterm children: neuropsychological correlates and predictors of attainment. Dev Neuropsychol 36, 74-95 (2011).

17 Sutendra, G. \& Michelakis, E. D. The chicken embryo as a model for ductus arteriosus developmental biology: cracking into new territory. Am J Physiol Regul Integr Comp Physiol 292, R481-484 (2007).

18 Ruijtenbeek, K., De Mey, J. G. \& Blanco, C. E. The chicken embryo in developmental physiology of the cardiovascular system: a traditional model with new possibilities. Am J Physiol Regul Integr Comp Physiol 283, R549550; author reply R550-541 (2002).

19 Speake, B. K., Murray, A. M. \& Noble, R. C. Transport and transformations of yolk lipids during development of the avian embryo. Prog Lipid Res 37, 1-32 (1998).

20 Nair, J., Gugino, S. F., Nielsen, L. C., Caty, M. G. \& Lakshminrusimha, S. Fetal and postnatal ovine mesenteric vascular reactivity. Pediatric research 79, 575-582 (2016).

21 Clough, G. F. Developmental conditioning of the vasculature. Compr Physiol 5, 397-438 (2015).

22 Russell, W.M.S. \& Burch, R.L. The Principles of Humane Experimental Technique. London: Methuen \& Co. Ltd. [Reissued: 1992, Universities Federation for Animal Welfare, Herts, England] (1959). 


\section{ABBREVIATIONS}

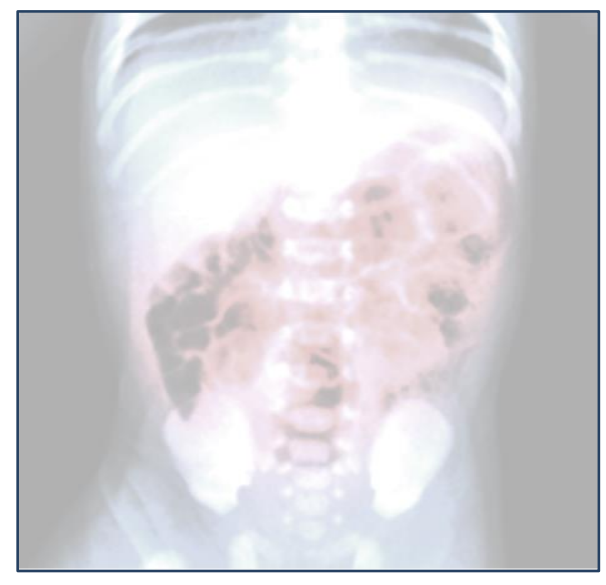




\section{LIST OF ABBREVIATIONS}

\begin{tabular}{|c|c|}
\hline $4-\mathrm{AP}$ & 4-aminopyridine \\
\hline $\mathrm{ACh}$ & acetylcholine \\
\hline ADMA & asymmetric dimethylarginine \\
\hline BPD & bronchopulmonary dysplasia \\
\hline BW & birth weight \\
\hline $\mathrm{CA}$ & chorioallantoic \\
\hline $\mathrm{Cl}$ & confidence interval \\
\hline CPS1 & carbamoyl-phosphate synthetase I \\
\hline $\mathrm{DA}$ & ductus arteriosus \\
\hline DMSO & dimethylsulfoxide \\
\hline EDHF & endothelium-derived hyperpolarizing factor \\
\hline $\mathrm{ET}-1$ & endothelin-1 \\
\hline GA & gestationa age \\
\hline HWE & Hardy-Weinberg equilibrium \\
\hline IUGR & intrauterine growth retardation \\
\hline $\mathrm{IVH}$ & intraventricular hemorrhage \\
\hline KRB & Krebs-Ringer bicarbonate buffer \\
\hline L-NAME & $\mathrm{N} \omega$-Nitro-L-arginine methyl ester \\
\hline LOS & late onset sepsis \\
\hline MA & mesenteric artery \\
\hline mETC & mitochondrial electron transport chain \\
\hline NE & norepinephrine \\
\hline NEC & necrotizing enterocolitis \\
\hline NO & nitric oxide \\
\hline NOS & nitric oxide synthase \\
\hline ODQ & $1 \mathrm{H}[1,2,4]$ oxadiazolo[4,3-a]quinoxalin-1-one \\
\hline OR & odds ratio \\
\hline PCR & polymerase chain reaction \\
\hline PDA & patent ductus arteriosus \\
\hline PROM & prolonged rupture of membranes \\
\hline PSS & physiological salt solution \\
\hline
\end{tabular}


PVL

periventricular leukomalacia

RDS respiratory distress syndrome

ROP retinopathy of prematurity

ROS

reactive oxygen species

SDMA

symmetric dimethylarginine

SERCA

sarcoplasmic reticulum $\mathrm{Ca} 2+$ ATPase

SGC

soluble guanylate cyclase

SNP

sodium nitroprusside

SNPS

single nucleotide polymorphisms

SOCC

store operated $\mathrm{Ca} 2+$ channel

SOD

superoxide dismutase

SSA

5-sulphosalicylic acid

TP

thromboxane/prostaglandin

VEGF vascular endothelial growth factor

VLBW very ow birth weight 
CO-AUTHORS

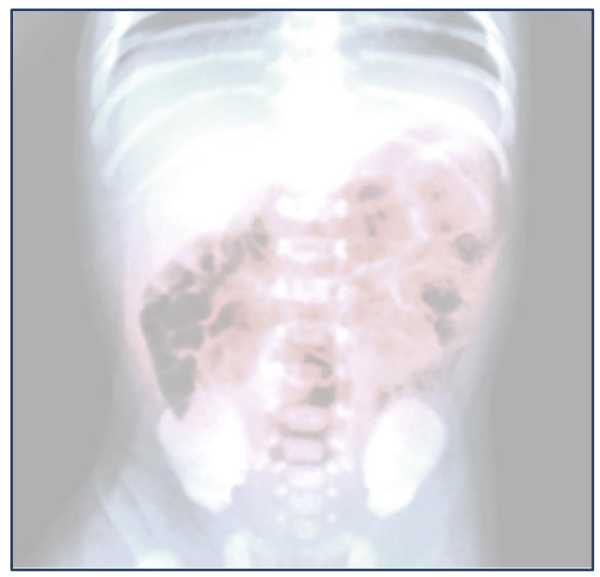




\section{LIST OF CO-AUTHORS}

Bakker, Jaap A. Department of Clinical Chemistry and Laboratory Medicine, Leiden University Medical Center, Leiden, The Netherlands

Barreira, Bianca Department of Pharmacology, School of Medicine, Universidad Complutense de Madrid, Centro de Investigaciones Biomédicas en Red de Enfermedades Respiratorias (CIBERES), Instituto de Investigación Sanitaria Gregorio Marañón (liSGM), Madrid, Spain

Brinks, Leonie Department of Pediatrics, Maastricht University Medical Center (MUMC+), School for Oncology and Developmental Biology (GROW), Maastricht, The Netherlands

Cavallaro, Giacomo Department of Clinical Sciences and Community Health, Fondazione IRCCS Cà Granda Ospedale Maggiore Policlinico, Università degli Studi di Milano, Milan, Italy

Cogolludo, Angel Department of Pharmacology, School of Medicine, Universidad Complutense de Madrid, Centro de Investigaciones Biomédicas en Red de Enfermedades Respiratorias (CIBERES), Instituto de Investigación Sanitaria Gregorio Marañón (liSGM), Madrid, Spain

González-Luis, Gema E. Department of Pediatrics, Hospital Universitario MaternoInfantil de Canarias, Las Palmas de Gran Canaria, Spain

Huizing, Maurice J. Department of Pediatrics, Maastricht University Medical Center (MUMC+), School for Oncology and Developmental Biology (GROW), Maastricht, The Netherlands

Kessels, Alfons G.H. Department of Clinical Epidemiology and MTA (KEMTA), Maastricht University Medical Center (MUMC+), Maastricht, The Netherlands 
Kessels, Carolina G.A. Department of Pediatrics, Maastricht University Medical Center (MUMC+), School for Oncology and Developmental Biology (GROW), Maastricht, The Netherlands

Moral-Sanz, Javier Department of Pharmacology, School of Medicine, Universidad Complutense de Madrid, Centro de Investigaciones Biomédicas en Red de Enfermedades Respiratorias (CIBERES), Instituto de Investigación Sanitaria Gregorio Marañón (liSGM), Madrid, Spain

Mosca, Fabio Department of Clinical Sciences and Community Health, Fondazione IRCCS Cà Granda Ospedale Maggiore Policlinico, Università degli Studi di Milano, Milan, Italy

Paulussen, Aimee D.C. Department of Clinical Genetics, Maastricht University Medical Center (MUMC+), Maastricht, The Netherlands

Perez-Vizcaino, Francisco Department of Pharmacology, School of Medicine, Universidad Complutense de Madrid, Centro de Investigaciones Biomédicas en Red de Enfermedades Respiratorias (CIBERES), Instituto de Investigación Sanitaria Gregorio Marañón (IISGM), Madrid, Spain

Reyes, Iballa Department of Pediatrics, Hospital Universitario Materno-Infantil de Canarias, Las Palmas de Gran Canaria, Spain

Rubio-Gozalbo, M. Estela Department of Pediatrics, Maastricht University Medical Center (MUMC+), Laboratory Genetic Metabolic Diseases, Maastricht, The Netherlands

Souren, Nicole Y.P. Department of Population Genetics, Maastricht University Medical Center (MUMC+), Nutrition and Toxicology Research Institute Maastricht (NUTRIM), Maastricht, The Netherlands 
Villamor, Eduardo Department of Pediatrics, Maastricht University Medical Center (MUMC+), School for Oncology and Developmental Biology (GROW), Maastricht, The Netherlands

Zimmermann, Luc J.I. Department of Pediatrics, Maastricht University Medical Center (MUMC+), School for Oncology and Developmental Biology (GROW), Maastricht, The Netherlands 


\section{DANKWOORD}

(Acknoweledgements)

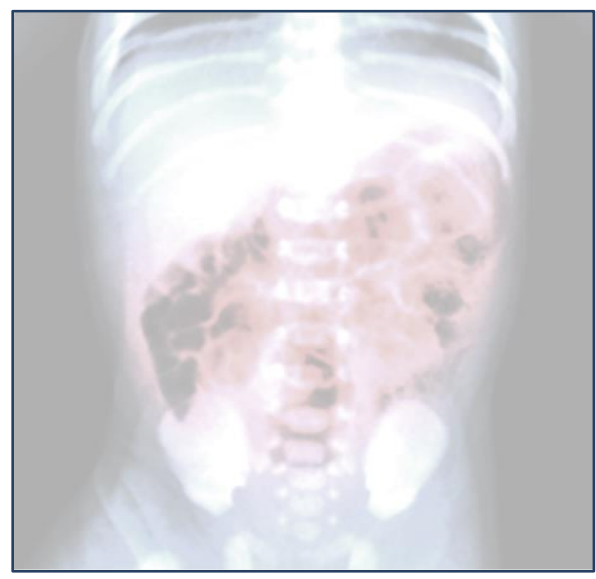




\section{DANKWOORD}

$\mathrm{Na}$ al die jaren van onderzoek naast een full time baan is het dan eindelijk zover, mijn proefschrift is klaar. Uiteraard heb ik het werk niet alleen gedaan. Er zijn vele mensen die mij door de jaren heen begeleid, geholpen en gesteund hebben. ledereen bedankt hiervoor!!!

Een aantal mensen wil ik hieronder in het bijzonder bedanken.

Allereerst bedankt aan alle ouders die toestemming gegeven hebben om een bloedmonster en wangslijmviesmonster af te laten nemen bij hun pasgeboren kind. Zonder deze kinderen was een deel van mijn proefschrift er niet geweest.

Ook een bijzonder woord van dank aan mijn promoteren Luc Zimmermann, Francisco Perez-Vizcaino en Boris Kramer. Beste Luc en Boris, bedankt voor de mogelijkheid en de steun die jullie me geboden hebben om te kunnen promoveren. Dear Francisco, 'muchas gracias' for the possibility of performing some of the expiriments in your lab in Madrid.

Deze promotie was er nooit van gekomen als Eduardo Villamor zich niet met heel zijn hart en ziel zou hebben ingezet voor het tot stand komen van dit proefschrift. Beste Eduardo, ik kon mij geen betere co-promotor wensen. Het heeft een tijd geduurd, maar nu is het klaar. Dank voor je onvoorwaardeijke steun gedurende de afgelopen jaren. Ik bewonder enorm je onuitputtelijke enthousiasme en energie bij het doen van wetenschappelijk onderzoek. Hiermee weet je iemand telkens weer te motiveren om door te gaan tot het einde. Naast co-promotor ben je ook een zeer gewaardeerd collega. We blijven in de toekomst verder samenwerken, zowel in de kliniek als bij wetenschappelijk onderzoek.

Ook wil ik de leden van de beoordelingscommissie, bestaande uit prof. dr. Frans Ramaekers, prof. dr. Edward Dompeling, prof. dr. Dino Giussani, prof. dr. Frits Prinzen en prof. dr. Máximo Vento, bedanken voor het lezen en beoordelen van mijn proefschrift. Dit geldt natuurlijk tevens voor de leden van de corona. 
Mijn dank gaat uit naar alle co-auteurs. Zonder hun bijdrage waren er geen artikelen om een proefschrift te maken. Specials thanks to Gema and Iballa for collecting samples in Spain and Giacomo for collecting samples in Italy.

Alle mensen op het laboratorium Kindergeneeskunde MUMC+ wil ik bedanken voor de fijne samenwerking. Lilian, Pia, Bea, Nico, Coen, Freek, Angel dank voor jullie expertise en gezelligheid. En natuurlijk dank aan de vele anderen wier naam ik niet genoemd heb, maar daardoor niet minder gewaardeerd.

Tante Riet en Ome Jan, dank om aan het einde van jullie vakantie bloed- en wangslijmvliesmonsters op te halen en met het vliegtuig vanuit Las Palmas de Gran Canaria mee naar Nederland te nemen.

Dank aan mijn collega's van de vakgroep Kindergeneeskunde voormalig Atrium medisch centrum Parkstad (nu Zuyderland medisch centrum, lokatie Heerlen) voor de extra tijd die ik ter beschikking heb gekregen voor dit onderzoek.

Dank aan mijn schoonfamilie en speciaal mijn schoonzus Pamela. Jij promoveerde 2 jaar geleden, dank voor de praktische tips en format van het proefschrift.

Mijn paranimfen, Jamiu Busari en Maurice Huizing, bedankt voor het terzijde staan op de dag van mijn promotie.

Ralph, beste broer, ben gewoon blij dat je in mijn leven bent.

Lieve mam en pap, als jullie me niet de kans hadden gegeven om te studeren, was dit proefschrift er zeker niet geweest. Jullie hebben ons altijd enorm gestimuleerd en gesteund. Ik ben heel trots dat jullie mijn ouders zijn, heel veel dank voor alles tot nu toe en wat nog gaat komen.

De allerbelangrijksten komen natuurlijk aan het eind van dit dankwoord. Lieve Sylvia, Lisa en Sanne heel veel dank voor jullie steun in de afgelopen jaren. Samen hebben we dit project van promoveren tot een goed einde gebracht. Geen uren schrijven meer, 
maar vanaf nu weer veel leuke dingen samen gaan doen. Het is niet in woorden uit te drukken hoe blij ik met jullie ben maar deze 4 komen wellicht in de buurt: IK HOU VAN JULLIE! 
ABOUT THE AUTHOR:

CURRICULUM VITAE AND PUBLICATIONS

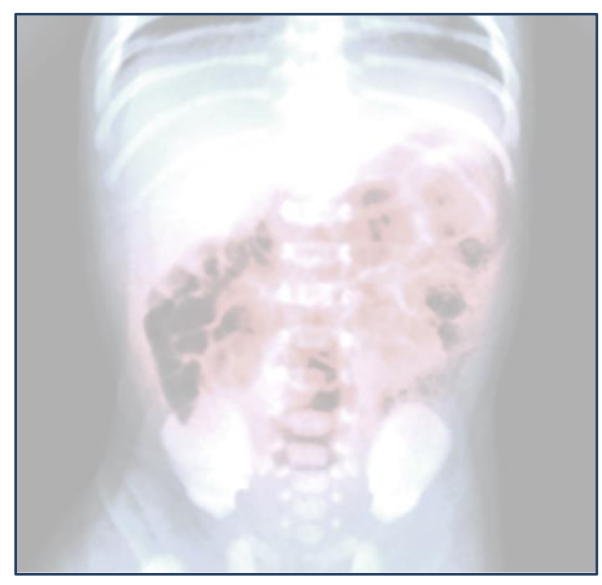




\section{CURRICULUM VITAE}

Rob Moonen was born on May 25th, 1975 in Heerlen and grew up in Brunssum. In 1993 he graduated from high school at the Romboutscollege in Brunssum. He started Medical school in 1993 at the Catholic University of Leuven where he obtained his degree in 2000. After graduation he worked as a pediatric resident (ANIOS) in the Maasland Hospital in Sittard. In February 2001 he started residential training in paediatrics in Atrium Medical Center Parkstad in Heerlen (supervisor: Dr. S.B. van der Meert) and Academic Hospital of Maastricht (supervisor: prof. dr. R.A.M.G. Donckerwolcke). After his training he started as a fellow Neonatology in August 2005 under supervision of prof. dr. L.J.I. Zimmermann and dr. A.L.M. Mulder and finished his fellowship February 2008. In this fellowship he initiated his PhD project under supervision of dr. E. Villamor. After his fellowship he worked a short period as paediatrician-neonatologist in the Academic Hospital of Maastricht. In August 2008 he started as an paediatrician-neonatologist in the Atrium Medical Center Parkstad in Heerlen (today named Zuyderland Medical Center). He is married to Sylvia Kleikers and they have two daughters, Lisa (2009) and Sanne (2012). 


\section{PUBLICATIONS}

\section{INCLUDED IN THIS THESIS}

Moonen RM, Paulussen AD, Souren NY, Kessels AG, Rubio-Gozalbo ME, Villamor E. Carbamoyl-phosphate synthetase polymorphisms as a risk factor for necrotizing enterocolitis. Pediatr Res 62:188-190 (2007).

Moonen RM, Reyes I, Cavallaro G, González-Luis G, Bakker JA, Villamor E. The T1405N Carbamoyl Phosphate Synthetase polymorphism does not affect plasma arginine concentrations and in preterm infants. PLoS One 5:e10792 (2010).

Moonen RM, Villamor E. Developmental changes in mesenteric artery reactivity in embryonic and newly hatched chicks. J Comp Physiol B 181:1063-1073 (2011).

Moonen RM, Kessels CG, Zimmermann LJ, Villamor E. Mesenteric Artery Reactivity and Small Intestine Morphology in a Chicken Model of Hypoxia-Induced Fetal Growth Restriction. J Physiol Pharmacol 63:601-612 (2012).

Brinks L, Moonen RM, Moral-Sanz J, Barreira B, Kessels L, Perez-Vizcaino F, Cogolludo A, Villamor E. Hypoxia-induced contraction of chicken embryo mesenteric arteries: mechanisms and developmental changes. Am J Physiol Regul Integr Comp Physiol 311:R858-R869 (2016).

Moonen RM, Cavallaro G, Huizing MJ, González-Luis GE, Mosca F, Villamor E. Association between the pTHR1406ASN Polymorphism of the Carbamoyl-Phosphate Synthase 1 gene and Necrotizing Enterocolitis: a Prospective Multicenter Study. Accepted for publication Sci Rep (2016 Oct 20 $\left.{ }^{\text {th }}\right)$. 


\section{NOT INCLUDED IN TIS THESIS}

R. Moonen, T. Avenant, K. De Boeck. Acute bacteriële thyreoïditis bij een 8 weken oude zuigeling. Tijdschrift voor Geneeskunde 57: 525-528 (2001).

R.M.J. Moonen, N.H.R.M. Peeters, P.J. Ribbink en P.M.V.M. Theunissen. ASTbloedglucosebepaling bij kinderen: een goed alternatief? EADVmagazine March 2003 20-22 (2003).

Moonen RM, Thissen CA, van der Kley AM, van der Meer SB. Behcet's disease in a Dutch boy with painful skin lesions. Ned Tijdschr Geneeskd 147:873-877 (2003).

Moonen RM, van der Meert SB. Diagnostic image (274). A neonate with a 'red eye'. Ned Tijdschr Geneeskd 150:1071 (2006).

van Hoorn JH, Moonen RM, Huysentruyt CJ, van Heurn LW, Offermans JP, Mulder AL. Pentalogy of Cantrell: Two patients and a review to determine prognostic factors for optimal approach. Eur J Pediatr 167:29-35 (2008).

Wolfs TG, Buurman WA, Zoer B, Moonen RM, Derikx JP, Thuijls G, Villamor E, Gantert M, Garnier Y, Zimmermann LJ, Kramer BW. Endotoxin induced chorioamnionitis prevents intestinal development during gestation in fetal sheep. PLOS ONE 4:e5837 (2009).

Moonen RM, Agren P, Cogolludo AL, Perez Vizcaino F, Villamor E. Responses of the Chicken Ductus Arteriosus to Hypercarbic and Normocarbic Acidosis. Neonatology 98:47-56 (2010).

van der Ham DP, Vijgen SM, Nijhuis JG, van Beek JJ, Opmeer BC, Mulder AL, Moonen R, Groenewout M, van Pampus MG, Mantel GD, Bloemenkamp KW, van Wijngaarden WJ, Sikkema M, Haak MC, Pernet PJ, Porath M, Molkenboer JF, Kuppens S, Kwee A, Kars ME, Woiski M, Weinans MJ, Wildschut HI, Akerboom BM, Mol BW, Willekes C; PPROMEXIL trial group. Induction of labor versus expectant 
management in women with preterm prelabor rupture of membranes between 34 and 37 weeks: a randomized controlled trial. PLoS Med 9:e1001208 (2012).

van der Ham DP, van der Heyden JL, Opmeer BC, Mulder AL, Moonen RM, van Beek JH, Franssen MT, Bloemenkamp KW, Sikkema JM, de Groot CJ, Porath M, Kwee A, Woiski MD, Duvekot JH, Akerboom BM, van Loon AJ, de Leeuw JW, Willekes C, Mol BW, Nijhuis JG. Management of late-preterm premature rupture of membranes: the PPROMEXIL-2 trial. Am J Obstet Gynecol 207:276.e1-10 (2012).

Busari JO, Stammen LA, Gennissen LM, Moonen RM. Evaluating medical residents as managers of care: a critical appraisal of assessment methods. Adv Med Educ Pract 5:27-37 (2014).

Wassen MM, Winkens B, Dorssers EM, Marcus MA, Moonen RM, Roumen FJ. Neonatal sepsis is mediated by maternal fever in labour epidural analgesia. $J$ Obstet Gynaecol 34:679-683 (2014).

Liesbeth C. Faverey, Rob M.J. Moonen. Een puber met hartkloppingen en pijn op de borst; meten is weten. Tijdschrift van de Belgische Kinderarts 16 - Nr.3 (2014).

Moonen RM, Huizing MJ, Cavallaro G, González-Luis GE, Bas-Suárez P, Bakker JA, Villamor E. Plasma levels of dimethylarginines in preterm very low birth weight neonates: its relation with perinatal factors and short-term outcome. Int J Mol Sci 16:1939 (2014). 
